\begin{abstract}
Title of Document:

Directed By:

Airframe Integrity Based on Bayesian Approach Jose Luis Hurtado-Cahuao, PhD, 2006.

Professor, Mohammad Modarres, Department of Mechanical Engineering.
\end{abstract}

Aircraft aging has become an immense challenge in terms of ensuring the safety of the fleet while controlling life cycle costs. One of the major concerns in aircraft structures is the development of fatigue cracks in the fastener holes. A probabilisticbased method has been proposed to manage this problem. In this research, the Bayes' theorem is used to assess airframe integrity by updating generic data with airframe inspection data while such data are compiled. This research discusses the methodology developed for assessment of loss of airframe integrity due to fatigue cracking in the fastener holes of an aging platform. The methodology requires a probability density function $(p d f)$ at the end of SAFE life. Subsequently, a crack growth regime begins. As the Bayesian analysis requires information of a prior initial crack size $p d f$, such a $p d f$ is assumed and verified to be lognormally distributed. The prior distribution of crack size as cracks grow is modeled through a combined Inverse Power Law (IPL) model and lognormal relationships. The first set of inspections is 
used as the evidence for updating the crack size distribution at the various stages of aircraft life. Moreover, the materials used in the structural part of the aircrafts have variations in their properties due to their calibration errors and machine alignment. A Matlab routine (PCGROW) is developed to calculate the crack distribution growth through three different crack growth models. As the first step, the material properties and the initial crack size are sampled. A standard Monte Carlo simulation is employed for this sampling process. At the corresponding aircraft age, the crack observed during the inspections, is used to update the crack size distribution and proceed in time. After the updating, it is possible to estimate the probability of structural failure as a function of flight hours for a given aircraft in the future. The results show very accurate and useful values related to the reliability and integrity of airframes in aging aircrafts. Inspection data shown in this dissertation are not the actual data from known aircrafts and are only used to demonstrate the methodologies. 


\section{AIRFRAME INTEGRITY BASED ON BAYESIAN APPROACH}

By

Jose Luis Hurtado Cahuao.

Dissertation submitted to the Faculty of the Graduate School of the University of Maryland, College Park, in partial fulfillment of the requirements for the degree of

Doctor of Philosophy 2006

Advisory Committee:

Professor Mohammed Modarres, Chair/Advisor

Dr. Paul Hoffman

Professor Emeritus Marvin Roush

Professor Ali Mosleh

Professor Bilal Ayyub

Professor Sreeramamurthy Ankem 
(C) Copyright by

Jose Luis Hurtado Cahuao

2006 


\section{DEDICATION}

This thesis is dedicated to my kids, Loredana and Robin whose support has proved invaluable to my research. 


\section{ACKNOWLEDGEMENTS}

There are many people to thank for their support and encouragement, without whom this thesis would not have been possible.

Firstly my advisor, Dr. Mohamed Modarres for discussions and guidance, and to give me the opportunity to work with him, whose enthusiasm for continue improving are probably the most valuable lessons I have learned from this $\mathrm{PhD}$, and his continual support and encouragement have kept me going over the last three or so years.

I would like to thank Dr. Paul Hoffman for providing technical guidance, resources and funding for my research assistantship throughout this $\mathrm{PhD}$. I would also like to thank Dr. Marvin Roush for introducing me to reliability engineering and supporting during my studies.

I would like to thank the many friends and colleagues who have helped in one way or another, especially Francisco Joglar for his understanding friendship, his contributions to the ideas developed herein, and her unstinting support. Reza Azarkhail, Genebelin Valbuena and Mohammad Pour-Gol-Mohamad for their technical assistance throughout the course of this thesis

Finally my family, who have been great over the years and never raised an eyebrow when I claimed my thesis would be finished in the "next month" for nearly a year. 


\section{TABLE OF CONTENTS}

LIST OF TABLES $\quad$ V

LIST OF FIGURES vii

LIST OF SUBSCRIPTS - xi

$\begin{array}{lll}\text { Chapter } 1 & \text { Introduction } & 1\end{array}$

1.1 Historical background 2

1.2 Technical Background 3

1.2.1 Stresses 3

1.2.2 Crack Size 4

1.2.3 Tensile Strength 4

1.2.4 Fatigue 5

1.2.5 Fatigue in the Aerospace Industry 5

$\begin{array}{lll}\text { 1.2.5.1 Safe-Life method } & 8\end{array}$

1.2.5.2 Fail-Safe method 8

1.2.5.3 Damage-Tolerance Method 9

1.3 Objectives 11

1.4 Motivation 11

1.5 Contributions 13

$\begin{array}{lll}1.6 & \text { Application } & 13\end{array}$

$\begin{array}{lll}\text { 1.6.1 Aircraft } & 13\end{array}$

$\begin{array}{ll}\text { 1.6.2 Analysis Location } & 14\end{array}$

$\begin{array}{lll}\text { 1.6.3 Materials } & 15\end{array}$

$\begin{array}{lll}1.6 .4 & 15 \\ 1.6 .5 & \text { Models } & 15\end{array}$

$\begin{array}{lll}1.6 .5 & \text { Structure } & 16\end{array}$

Chapter 2 Technical Description of Fatigue Crack Growth / Material Properties 19

2.1 Fatigue Crack Growth Phenomenon 19

2.2 Linear Elastic Fracture Mechanics 24

2.2.1 Stress Intensity Factor 25

2.2.2 Crack tip Plastic Zone Size 28

$\begin{array}{lll}2.2 .3 & \text { Fracture Toughness } & 30\end{array}$

2.3 Sigmoidal Shape d $a / \mathrm{d} N$ Curve 33

2.4 Effect of Stress Ratio Stress on fatigue Crack Growth 36

2.5 Effects of complex time history loads on fatigue crack growth 37

2.6 Material Properties 40 
2.6.1 Mechanical Properties $\quad 40$

2.6.2 Elastic modulus $(E) \quad 42$

2.6.3 Yield strength $\left(S_{\mathrm{y}}\right) \quad 42$

2.6.4 Ultimate strength $\left(S_{\text {ult }}\right) \quad 44$

Chapter 3 Data Collection $\quad 47$

3.1 Load Spectrum - Background 47

3.2 Calculation of Spectrum Load 49

3.2.1 Fatigue Life Expended Index (FLE) 54

3.3 Initial Crack Size Distribution 55

3.3.1 Equivalent Initial Flaw Size 56

3.3.2 Time-To-Crack Initiation 56

3.4 Crack Observation (Evidence) 59

Chapter 4 An Integrated Approach for Assessing the Crack Growth Under Variable Amplitude Loading

4.1 Crack Growth Assessment 63

$\begin{array}{lll}\text { 4.1.1 Global Approach } & 64\end{array}$

4.1.2 Cycle-by-Cycle Approach 65

4.2 Non-interaction models $\quad 67$

$\begin{array}{lll}\text { 4.2.1 Walker Model } & 67\end{array}$

$\begin{array}{lll}\text { 4.2.2 Forman Model } & 70\end{array}$

4.3 Interaction model: Closure Model 73

$\begin{array}{lll}4.4 & \text { Failure Criteria } & 78\end{array}$

4.5 Crack Growth Estimation and Uncertainty Propagation 79

4.6 Model Verification $\quad 81$

Chapter 5 Probabilistic Parameter Estimation of the Models. 88

5.1 Lognormal Distribution as a Representation of Crack Size Distribution. 90

5.2 Inverse Power Law Relationship (IPL) as a Representation of Life (life expended) and Crack Size. 93

5.3 IPL -Lognormal Relationship. $\quad 94$

5.4 Parameter Estimation Using MLE 97

5.5 Estimation of the Prior Distribution of Crack Size as Crack Grows for Bayesian Parameter Estimation 99

5.6 Markov Chain Monte Carlo (MCMC) 102 
$\begin{array}{lll}\text { 5.6.1 Monte Carlo integration } & 102\end{array}$

5.6.2 Metropolis-Hasting and Gibbs Sampling 103

$\begin{array}{lll}\text { 5.6.2.1 Burn-in period } & 106\end{array}$

$\begin{array}{lll}5.7 & \text { Genetic Algorithm (GA) } & 107\end{array}$

Chapter 6 Bayesian Updating Process of the Crack Size Distribution 117

6.1 Bayesian Updating of Crack Size Distribution 118

6.2 Posterior $p d f$ for $F L E=100 \% \quad 125$

6.2.1 Posterior Initial Crack Size $p d f$ for $F L E=100 \% \quad 128$

$\begin{array}{ll}\text { 6.2.2 Posterior Model Parameters } & 133\end{array}$

6.2.3 Posterior Initial Crack Size $p d f$ for $F L E=100 \%-\mathrm{MCMC} \quad 138$

6.3 Forecasting Probability of Failure for a Specific $F L E_{j} \% \quad 140$

$\begin{array}{lll}6.4 & \text { Decision Making Methodology } & 141\end{array}$

$\begin{array}{lll}\text { Chapter } 7 \text { Analysis of the results } & 146\end{array}$

$\begin{array}{lll}7.1 & \text { Monte Carlo Simulation } & 146\end{array}$

$\begin{array}{lll}7.2 & \text { Crack Growth Model Comparisons } & 147\end{array}$

$\begin{array}{lll}7.3 & \text { Selection of Lognormal Distribution } & 148\end{array}$

$\begin{array}{lll}7.4 & \text { Parameter Estimation } & 149\end{array}$

$\begin{array}{lll}7.5 & \text { Bayesian Analysis } & 150\end{array}$

$\begin{array}{lll}7.6 & \text { Decision Making } & 155\end{array}$

$\begin{array}{lll}\text { Chapter } 8 \text { Conclusions \& Recommendations } & 165\end{array}$

Appendix A PCGROW code for crack growth -closure model example $\begin{array}{ll}\text { calculation. } & 169\end{array}$

Appendix B WinBUGS code for parameter estimation example calculation. 176

$\begin{array}{ll}\text { References } & 178\end{array}$ 


\section{LIST OF TABLES}

Table 1.1 Parameters of crack growth models. 16

Table 2.1 Typical cases of fatigue problems. 20

Table 2.2 Characteristics of the three regimes of fatigue crack growth [2]. 35

Table 2.3 Properties for the aluminum 7075-T651. 45

Table 3.1 Flight and stresses description. $\quad 52$

Table 3.2 Spectrum summary for the 25 aircrafts. 53

Table 3.3 Observed number of various crack sizes for the 25 aircrafts. 62

$\begin{array}{ll}\text { Table 4.1 Walker parameters. } & 70\end{array}$

Table 4.2 Forman Parameters.

Table 4.3 Crack growth lookup table for 7076-T6 [75]. 77

Table 4.4 Materials properties for the 7057-T6. 78

Table 4.5 Beta values. 83

Table 5.1 Goodness of fit results. 92

Table 5.2 IPL-Lognormal mean parameters at the FLE showed - Based on

Walker crack growth model.

Table 5.3. IPL-Lognormal mean parameters at the FLE showed - Based on

$\begin{array}{ll}\text { Forman crack growth model. } & 100\end{array}$

Table 5.4 IPL-Lognormal mean parameters at the FLE showed - Based on

$\begin{array}{ll}\text { Closure crack growth model. } & 101\end{array}$

Table 5.5 IPL-Lognormal mean parameters values using three estimation

$\begin{array}{ll}\text { methodologies. } & 115\end{array}$

Table 6.1 Updated Lognormal Parameters for the Three Crack Growth models -

Conjugate.

Table 6.2 Lognormal distribution parameters at $F L E_{j \%}$ for each aircraft - MCMC. 124

Table 6.3 Model parameters updating. 138

Table 7.1 Differences between Conjugate and MCMC approach. 153

Table 7.2 Initial crack size Conjugate and MCMC at 100\% FLE. 154

Table 7.3 Prediction results $\Delta \mathrm{FH}=1000$ - crack size XS. 156 
Table 7.4 Prediction results $\Delta \mathrm{FH}=1000$ - crack size $\mathrm{S}$.

Table 7.5 Prediction results $\Delta \mathrm{FH}=1000$ - crack size L. 158

Table 7.6 Prediction results $\Delta \mathrm{FH}=1000$ - crack size XL. 159

Table 7.7 Aircrafts to maintenance. 163

Table 7.8 Aircrafts to maintenance after the box plot analysis. 163 


\section{LIST OF FIGURES}

Figure 1.1 World fatal accident numbers and fatal accident rates 1978 -98. Large Civil Jet Transport aircraft Data [4]. 3

Figure 1.2 Crack on the skin fillet at the wing root of a F-100 aircraft [16]. 7

Figure 1.3 Layout of the part, and the location where the analysis was carried out. 14

$\begin{array}{ll}\text { Figure 1.4 Structure of the dissertation. } & 18\end{array}$

Figure 2.1 Schematic curves for both finite and infinite life [28]. 21

Figure 2.2 Fatigue life [28]. 22

Figure 2.3 Effect of surface finish on the pre-crack life and the crack propagation life of unnotched rotating beam specimens of $0.2 \%$ C steel (SAE 1020) [28]. 23

Figure 2.4 Approach followed in the research. 24

Figure 2.5 Elastic stresses near the crack tip $(\mathrm{r} / \mathrm{a}<<1)[2]$.

Figure 2.6 $\beta$ factor for the growth towards small rivet holes. 27

Figure $2.7 \beta$ factor for the growth from satellite rivet holes. 28

Figure 2.8 Plastic zone size at the tip of a through thickness crack [2]. 29

Figure 2.9 Effect of specimen thickness on fracture toughness [2]. 31

Figure 2.10 Fatigue crack length vs. applied cycles. Fracture is indicated by X [2]. $\quad 32$

Figure 2.11 Fatigue crack growth rate behavior [34]. 34

Figure 2.12. Schematic mean stress influence on fatigue crack growth rates [2]. 36

Figure 2.13 A schematic illustration of transient crack growth during constant

and variable amplitude [1]. 38

Figure 2.14 Crack propagation under simulated flight loading, 2024-T3 alum [37].

Figure 2.15 Variation of material properties [41].

Figure 3.1 Gust load spectra measured with counting accelerometers compared to the design load spectrum of the Fokker F-28 [33]. 49

Figure 3.2 Mission profile [45]. 50

Figure 3.3 Simplified load spectrum for an aircraft [45]. 50 
Figure 3.4 Dome nut hole locations.

Figure 3.5 Process showing the compatibility between time-to-crack initiation and initial flaw size $p d f[42]$. 58

Figure 3.6 Estimated Initial Crack Size $p d f$.

Figure 3.7 Distribution of cracks found in service [58]. 60

Figure 3.8 Cracks location at the FC 351 area. 61

$\begin{array}{lll}\text { Figure } 3.9 \text { Inspection cracks size. } & 61\end{array}$

$\begin{array}{lll}\text { Figure 4.1 Life prediction models. } & 64\end{array}$

Figure 4.2 Logic diagram for damage crack growth. 66

Figure 4.3 Crack growth in 2024-T3 and 7075-T6 [60]. 69

Figure 4.4 Comparison of experimental and theoretical crack-propagation rates in 7075-T6 aluminum plate for $R=0$ to $R=0.15$ [65]. 72

Figure 4.5 Crack closure concept [37].

Figure 4.6 Stress intensity Factor for the 7075-T6 aluminum [31]. 79

Figure 4.7 Crack growth and uncertainty propagation algorithm. 80

Figure 4.8 Example of Random Rack Growth trajectories at 231\% FLE. 81

Figure 4.9 Model geometry and dimension in AFGROW. 82

Figure 4.10 Spectrum profile. 83

Figure 4.11 PCGROW and AFGROW comparison - Closure. 84

Figure 4.12 Overload and underload spectrum. 85

Figure 4.13 Results under overload-underload amplitude. 85

Figure 4.14 PCGROW and AFGROW comparison - Walker. 86

Figure 4.15 PCGROW and AFGROW comparison - Forman. 87

Figure 5.1 Underlying life distribution and stress-life relationship at different stress levels. $\quad 88$

Figure $5.2 p d f$ vs. time and stress. [80]. 89

Figure 5.3 Lognormal fit for 7075 aluminum specimens under six different stress levels [2].

Figure 5.4 Lognormal fit for 7075 aluminum specimens [2]. 91

Figure 5.5 Lognormal and Weibull graphical comparison. 93

Figure 5.6 A three dimensional representation of the $p d f$ vs. crack size and FLE. 96 


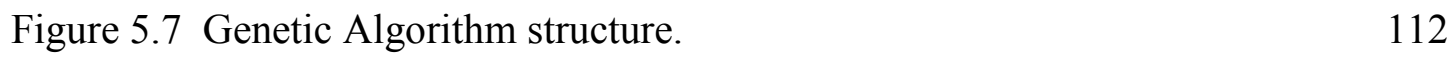

Figure 5.8 Likelihood and average likelihood for aircraft 2.

Figure 5.9 Likelihood and average likelihood for aircraft 18.

Figure 6.1 Updating process at the FLE of interest. 120

$\begin{array}{ll}\text { Figure 6.2 Crack size ranges. } & 121\end{array}$

Figure 6.3 Bayesian Updating Results. 123

Figure 6.4 Updating Prior Crack Size Distributions. 126

Figure 6.5 Algorithm used in PCGROW code for the parameters updating. 127

Figure 6.6 Updated distributions at 100\% FLE. 128

Figure 6.7 Method of moments. 129

Figure 6.8 Prior \& Posterior crack size $p d f, F L E=100 \%$ - Walker model. $\quad 130$

Figure 6.9 Prior \& Posterior crack size $p d f, F L E=100 \%$ - Forman model. 130

Figure 6.10 Prior \& Posterior crack size $p d f, F L E=100 \%$ - Closure model. $\quad 131$

Figure 6.11 Posterior versus smooth crack size $p d f, F L E=100 \%$ - Walker model 131

Figure 6.12 Posterior versus smooth crack size $p d f$, $F L E=100 \%$ - Forman model 132

Figure 6.13 Posterior versus smooth crack size pdf, $F L E=100 \%$ - Closure

$\begin{array}{ll}\text { model. } & 132\end{array}$

Figure $6.14 m_{1}$ parameter. $p d f, F L E=100 \%$. 133

Figure $6.15 C_{1}^{*}$ parameter $p d f, F L E=100 \% . \quad 134$

Figure $6.16 \gamma$ parameter. $p d f, F L E=100 \% . \quad 134$

Figure $6.17 m_{2}$ parameter. $p d f, F L E=100 \%$. 135

Figure $6.18 C_{2}$ parameter. $p d f, F L E=100 \%$. 135

Figure $6.19 K_{\mathrm{C}}$ parameter. $p d f, F L E=100 \% . \quad 136$

Figure $6.20 S_{\mathrm{y}}$ parameter. $p d f, F L E=100 \%$. 136

Figure $6.21 S_{\text {ult }}$ parameter. $p d f, F L E=100 \% . \quad 137$

Figure $6.22 E$ parameter. $p d f, F L E=100 \% . \quad 137$

Figure 6.23 Posterior versus smooth crack size $p d f$, FLE $=100 \%$ - Walker model, $\begin{array}{ll}\text { MCMC approach. } & 139\end{array}$

Figure 6.24 Posterior versus smooth crack size $p d f$, FLE $=100 \%$ - Forman model, MCMC approach. 
Figure 6.25 Posterior versus smooth crack size $p d f, F L E=100 \%$ - Closure model, MCMC approach. 140

Figure 6.26 Forecasting process for a specific $F L E_{j \%}$. 141

Figure 6.27 Model uncertainty analysis. 143

Figure 7.1 Examples of random crack growth trajectories at 297\% FLE. 147

Figure 7.2 Crack growth vs. FLE. 148

Figure 7.3 Example of lognormal fit for aircraft $4 . \quad 149$

Figure 7.4 Comparison of parameter estimation methodologies. 150

Figure 7.5 Examples of parameter estimation. 150

Figure 7.6 Prior and post for two aircraft evidential datasets (4 cracks size "XS") and (1 crack size "XS", 1 crack size "S".)

Figure 7.7 Prior and post for two aircraft evidential datasets ( 7 cracks size "S") and (5 cracks size "XS", 2 cracks size "S", 2 cracks size "L".)

Figure 7.8 initial crack size for aircrafts 12 and 22 using conjugate and MCMC approach.

Figure 7.9 Initial crack size at 100\% FLE for the three models. 154

Figure 7.10 Example of posterior $p d f$ crack growth models parameter. 155

Figure 7.11 Box plot of the three models at $\Delta \mathrm{FH}=1000$ - Aircrafts 1 to $9 . \quad 160$

Figure 7.12 Box plot of the three models at $\Delta \mathrm{FH}=1000$ - Aircrafts 10 to $18 . \quad 160$

Figure 7.13 Box plot of the three models at $\Delta \mathrm{FH}=1000$ - Aircrafts 19 to $25 . \quad 161$

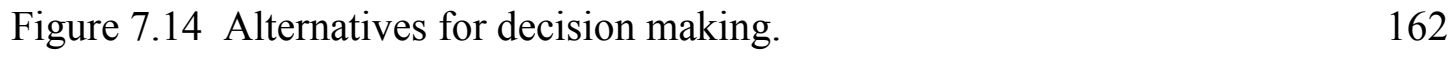




\section{LIST OF SUBSCRIPTS}

\begin{tabular}{|c|c|}
\hline Subscripts & Description \\
\hline$a$ & Crack size. \\
\hline$a_{\mathrm{c}}$ & Crack length at instability. \\
\hline$C$ & Paris experimentally estimated constants. \\
\hline$C_{1}$ & Walker model parameter. \\
\hline$C_{2}$ & Forman model parameter. \\
\hline $\mathrm{d} a / \mathrm{d} N$ & Crack growth rate. \\
\hline$E$ & Elastic modulus. \\
\hline $\mathrm{EF}$ & Error factor. \\
\hline $\mathrm{FH}$ & Flight hours. \\
\hline FLE & Fatigue life expended \\
\hline FSFT & Full-scale fatigue test. \\
\hline GA & Genetic algorithm. \\
\hline IPL & Inverse power law. \\
\hline$K$ & Stress intensity factor. \\
\hline$k$ & IPL parameter \\
\hline$K_{\mathrm{C}}$ & Fracture toughness \\
\hline$K_{\mathrm{IC}}$ & Plane strain fracture toughness. \\
\hline$K_{\text {op }}$ & Opening stress intensity factor. \\
\hline LEFM & Linear elastic fracture mechanics. \\
\hline$m$ & Paris experimentally estimated constants. \\
\hline$m_{1}$ & Walker model parameter. \\
\hline$m_{2}$ & Forman model parameter. \\
\hline $\mathrm{MCMC}$ & Markov chain monte carlo. \\
\hline MLE & Maximum likelihood estimator. \\
\hline
\end{tabular}




\begin{tabular}{ll}
$n$ & Second IPL parameter. \\
NDI & Non destructive inspection. \\
PCGROW & Probabilistic crack growth. \\
$R$ & Stress ratio. \\
$S$ & Far field stress. \\
SAFE & Structural Appraisal of Fatigue Effects. \\
$S_{\mathrm{C}}$ & Applied nominal stress at crack instability. \\
$S_{f l o w}$ & Flow stress. \\
$S_{\mathrm{op}}$ & Stress opening. \\
$S_{\mathrm{ult}}$ & Ultimate strength. \\
$S_{\mathrm{y}}$ & Yield strength \\
$U$ & Effective stress ratio. \\
WFD & Widespread fatigue damage. \\
$\delta_{0}$ & Parameter of the prior distribution. \\
$\delta_{*}$ & Parameter of the likelihood distribution. \\
$\Delta K$ & Stress intensity factor range. \\
$\Delta S_{\text {eff }}$ & Effective stress range. \\
$\alpha$ & Constraint factor. \\
$\beta$ & Beta geometric factor \\
$\delta^{\prime}$ & Parameter of the posterior distribution. \\
$\gamma$ & Walker model parameter. \\
$\lambda^{\prime}$ & Parameter of the posterior distribution. \\
$\lambda_{0}$ & Parameter of the prior distribution. \\
$\lambda_{*}$ & Parameter of the likelihood distribution. \\
$\sigma_{a}$ & Standard deviation of the natural logarithm of the crack \\
& growth rate \\
\hline &
\end{tabular}




\section{Chapter 1 Introduction}

The word fatigue originated from the Latin expression "fatigare", which means "to tire" [1]. Although commonly associated with physical and mental weariness in people, the word fatigue also became a widely accepted term in engineering vocabulary when referring to the damage and failure of materials under cyclic loads. From an engineering perspective, the American Society of Testing Material (ASTM) defines fatigue as: "the process of progressive localized permanent structural change occurring in a material subjected to conditions which produce fluctuating stresses and strains at some point or points and which may culminate in cracks or complete fracture after a sufficient number of fluctuations" [2].

The design of many structures in aeronautical engineering (aircraft structures), mechanical engineering (pressure vessels, piping, etc.), and civil engineering (bridges, offshore structures, nuclear power plants, etc.) include provisions to prevent fatigue related failures. At the same time, the prediction of fatigue related failures is still an active subject of research, which can help design, and maintain these structures. In relation with aircraft structures (which is the focus of this research), fatigue still plays an important role in aging aircrafts, (that is those over 15 years old.) Many of these aircrafts have accumulated flight hours approaching and in many cases exceeding the original design. The statistics show that the number of aging commercial aircrafts (older than 15 years) has increased continuously. This number was around 4600 in 1997 for US and European built civil aircrafts flown with more than 1900 aircrafts older than 25 years. This number increased to 4730 ( $>15$ years) and 2130 (>25 years) respectively in 1999 [3]. The same can be seen with military 
aircraft, where an increasing number of aircrafts (e.g. F-14, T-38, MiG21) now exceed their anticipated life of 40 years. Looking at ongoing mid-life updates of fighter airplanes, service lives of 50 years and more are not exceptional. Even in the civil aircraft world nearly half of the whole DC-8 fleet is still flying [4].

\subsection{Historical background}

Fatigue has been recognized as an important failure mode in the aircraft industry from the very beginning. Even before the Wright brother's first flight in 1903, a fatigue failure in an engine shaft delayed the first flight attempt while a new shaft was manufactured. In the succeeding century, fatigue failures have been brought under control to the extent that fatal accidents resulting from failure of the aircraft structure occur approximately once every 100 million flights [3]. This low rate arises not only from technologies, which have eliminated fatigue; but also from acknowledging that fatigue damage is inevitable, that aircraft structures contain flaws, and that most accurately calculated lives may be uncertain.

Despite the growth in air transport passenger hours of about $6 \%$ per year, civil aircraft accident numbers have remained roughly constant over the past 20 years between 20-30 per year worldwide [5]. As the number of flights has increased, the number of fatal accidents per flight has reduced in the same period, from 1 in every 10 million flights in 1980 to about 1 in every 30 million flights today. Figure 1.1 shows a considerable year-to-year variation in the fatal accident rate. It also shows that the trend is generally downward. 


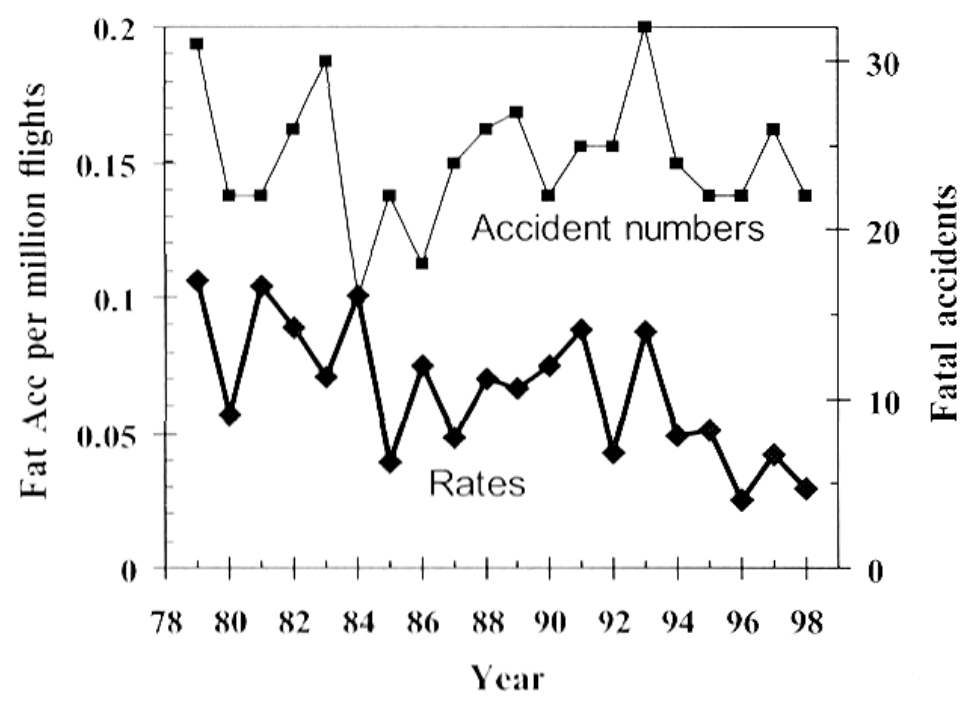

Figure 1.1 World fatal accident numbers and fatal accident rates 1978 -98. Large Civil Jet Transport aircraft Data [4].

\subsection{Technical Background}

Fatigue of materials is still only partly understood. The available knowledge has been developed in stages and has become quite complex. Consider the following brief historical review of fatigue developments which demonstrate a few basic concepts and briefly indicates their development.

\subsubsection{Stresses}

The first major impact of failures due to repeated stresses affected the railway industry in the 1840 s. Consequently, in Germany during the 1850 s and 1860s, August Wohler performed many laboratory fatigue tests under repeated stresses. These experiments were concerned with railway axle failures and are 
considered to be the first systematic investigation of fatigue. Thus, Wohler has been called the "father" of systematic fatigue testing. He also introduced the concept of the $S-N$ diagram and the fatigue limit, and pointed out that the range of stresses is more important than the maximum stress [6]. During the 1870s and 1890s additional researchers substantiated and expanded Wohler's classical work. Gerber [2], along with others, investigated the influence of mean stress. In addition, Goodman [2] proposed a simplified theory concerning mean stresses.

\subsubsection{Crack Size}

In 1920 Griffith [7] published the results of his theoretical calculations and experiments on brittle fracture using glass. He found the strength of glass depended on the size of microscopic cracks. He showed that if $S$ is the nominal stress

at fracture and $a$ is the crack size at fracture, the relation $\mathrm{s} \sqrt{a}$ is constant. For this classical pioneering work on the importance of cracks, Griffith is known as the "father" of fracture mechanics.

\subsubsection{Tensile Strength}

In 1929-30 Haigh [8] presented his rational explanation of the difference in the response of high tensile strength steel and of mild steel to fatigue when notches are present. He used concepts of notch strain analysis and self-stresses that were later more fully developed by others. In 1937, Neuber introduced stress gradient effects at notches and the elementary block concept, which considers that the average stress over a small volume at the root of the notch is more important than the peak stress at the notch. 


\subsubsection{Fatigue}

During World War II the deliberate use of compressive self-stresses became common in the design of aircraft engines and armored vehicles. Many brittle fractures in welded tankers and in Liberty ships motivated substantial efforts and thinking concerning preexisting defects in the form of cracks and the influence of stress concentrations. Many of these brittle fractures started at square hatch corners or square cutouts and welds. Solutions included rounding and strengthening corners, adding riveted crack arresters, and greater emphasis on material properties. In 1945 Miner [9] formulated a linear cumulative fatigue damage criterion suggested by Palmgren [10] in 1924. This linear fatigue damage criterion is now recognized as the Palmgren-Miner rule. It has been used extensively in fatigue design and, despite its many shortcomings, still remains an important tool in fatigue life predictions.

\subsubsection{Fatigue in the Aerospace Industry}

The Comet, the first jet propelled passenger airplane, started service in May 1952 after more than 300 hours of flight tests. Four days after an inspection in January 1954 it crashed into the Mediterranean Sea. After much of the wreckage had been recovered from the bottom of the sea and exhaustive investigation and tests on components of Comet aircraft was made, it was concluded that the accident was caused by fatigue failure of the pressurized cabin. The small fatigue cracks originated from a corner of an opening in the fuselage.

Two Comet aircrafts failed catastrophically. Probably the first 30 high load levels induced sufficient self (residual) stresses in the test section so as to falsely 
enhance the fatigue life of the test component and provide overconfidence. As a result, all Comet aircrafts of this type were taken out of service

The fatigue induced failures in the Comet aircrafts significantly increased the attention in preventing this type of failure mode. For example, Irwin [11] introduced the stress intensity factor $K_{\mathrm{I}}$, which has been accepted as the basis of linear elastic fracture mechanics (LEFM) and of fatigue crack growth life predictions.

Subsequently, in the early 1960s low cycle strain-controlled fatigue behavior became prominent with the Manson-Coffin $[12,13]$ relationship between plastic strain amplitude and fatigue life. These ideas are the basis for current notch strain fatigue analysis. Also in the early 1960's Paris [14] showed that fatigue crack growth rate $\mathrm{d} a / \mathrm{d} N$ could best be described using the stress intensity factor range $\Delta K_{\mathrm{I}}$. In the late 1960s the catastrophic crashes of F-111 aircrafts were attributed to brittle fracture of members containing preexisting flaws. These failures, along with fatigue problems in other U.S. Air Force planes, laid the groundwork for the requirements to use fracture mechanics concepts in the B-1 bomber development program of the 1970s. This program included fatigue crack growth life considerations based on a pre-established detectable initial crack size. In July 1974 the U.S. Air Force issued Mil A-83444, defining damage tolerance requirements for the design of new military aircrafts. At this point, the use of fracture mechanics as a tool for fatigue was thus thoroughly established through practice and through regulations.

The aftermath of the 1988 Aloha Airlines flight 243 incidents, in which a portion of the passenger compartments disintegrated during a short flight, forced the 
aerospace community to reexamining the procedure developed to ensure the structural integrity of aircraft for civilians and military aircrafts. The committee that investigated the Aloha airlines incident attributed the failure to the sudden linking of multiple undetected cracks at and around rivet holes in the metallic panels comprising the skin of the pressurized fuselage. This in-service incident unveiled the potential threat to airframe structural integrity caused by the interaction and uncontrolled linkup of seemingly small and often undetectable cracks in riveted primary structure [15]. This type of fatigue damage, often referred to as widespread fatigue damage (WFD), is characteristic of the large population of aging aircraft. Figure 1.2 shows an example of fatigue crack in a fuselage splice joint of a transport aircraft.

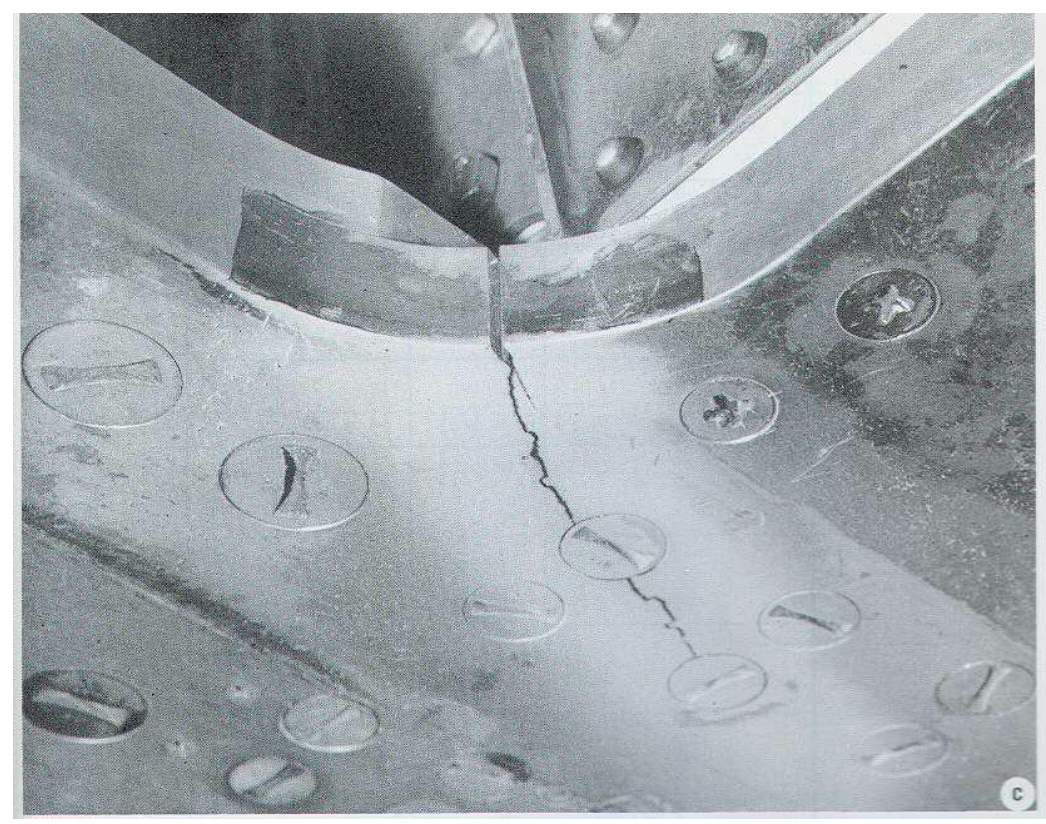

Figure 1.2 Crack on the skin fillet at the wing root of a F-100 aircraft [16].

Aircraft structural engineers have used several methods to determine the life of aircraft. The following methods are the important ones: 


\subsubsection{Safe-Life method}

The safe-life method predicts a replacement time for aircraft components, usually specified as a number of allowable landings or flight hours. The replacement time based upon the time required for failure, which is obtained from component fatigue tests. In most cases, a component is designed so that the replacement time for that component exceeds the expected service life of the aircraft. Once a component reaches its replacement time, its safe-life is considered to be used up and it is retired, regardless of whether any fatigue cracks are present. Ideally, a component designed according to safe-life principles will be replaced before it develops a fatigue crack. There were, however, two significant problems inherent in this method [17]:

- the safety of an aircraft was not protected if it contained a manufacturing or maintenance induced defect, and

- retirement times were not related to statistically-based safety factors. To maximize safety, the selected safety factor had to be conservative. As a result, many components were prematurely retired.

\subsubsection{Fail-Safe method}

The fail-safe method to aircraft fatigue design was developed during the 1960s and implemented in a number of commercial aircraft [18]. The goal of the fail-safe philosophy is to design multiple load path structures, such that if an individual element should fail, the remaining 
elements would have sufficient structural integrity to carry the additional loads from the failed element until the damage is detected through scheduled maintenance inspections. In addition to multiple load path structures, crack stoppers are also commonly used in fail-safe designs. Crack stoppers, which typically consist of materials with high fracture toughness, are used to supplement the residual strength of surrounding structure and prevent cracks from propagating to failure [17]. An example of a crack stopper is a stringer in a pressurized fuselage. The stringer reduces the amount of energy available for crack growth, slowing or stopping the advance of a crack that crosses it. Ideally, an aircraft designed according to fail-safe principles can sustain damage and remain airworthy until the damage is detected and repaired. This necessitates periodic inspections to examine the structure to determine if the primary load carrying member contains cracks. The frequency of these inspections is typically assigned by the manufacturer based upon service experience. A limitation of this method is that it does not consider the initiation and growth (linking up) of small cracks at fastener holes. As a result, the loss of several "fail-safe" aircrafts in the mid-1970s emphasized the need to locate cracks and repair damage before failure occurred [19].

\subsubsection{Damage-Tolerance Method}

Based upon fracture mechanics techniques, the damage tolerance approach redefined the basis for analyzing fatigue cracks in aircraft

structures. With economic and safety advantages over the previous 
methods, the damage-tolerance philosophy was eventually adopted by the commercial aircraft industry as well $[20,21]$. The objective of the damage tolerance approach is to detect cracks in principal structural elements before they propagate to failure. A principal structural element (PSE) is defined as any aircraft structure carrying flight, ground, or pressurization loads, whose failure could result in the loss of the aircraft [17]. By establishing inspection intervals for these elements based upon the time it takes a crack to grow from an initial detectable size to the critical crack length, the objective of the damage-tolerance approach can be achieved. Unlike the safe-life approach where components are retired whether or not they are damaged, components are only replaced if a crack is found during an inspection. It is important to note that, although the detectable crack size dictates the time between inspections to a certain degree, any size crack found during an inspection mandates replacement of the damaged component.

A limitation of this method is lack of consideration of uncertainties. That is the developers of the damage tolerance requirements make deterministic rather than probabilistic assessment of, specific load numbers and the critical length of cracks [22]. Lately, some researchers have proposed an Initial Flaw Size Distribution, which is obtained from tests that determine the distribution of times it takes a crack of some initial size to reach a specified reference size [23]. 
Furthermore, because of the inherent fatigue strength variability of materials as well as the statistical nature of the service loads experienced by the structures, the uncertainty consideration is essential to the fatigue life prediction.

\subsection{Objectives}

Based on the limitations mentioned above, the main objectives of this dissertation are:

1. To review the probabilistic approaches to fatigue crack growth based on the concept of Linear Elastic Fracture Mechanics (LEFM).

2. To assess the uncertainty about the crack size distribution through of the Bayesian approach, which provides a mechanism of updating one's degree of belief about a proposition (e.g. crack size) in light of new evidence (e.g. inspection results).

3. To estimate the probability of structural failure after a number of flight hours.

4. To account for crack growth model uncertainty by considering three different models in order to give the decision makers more information to make a riskinformed decision.

5. To demonstrate the methodology on a specific aging aircraft fleet.

\subsection{Motivation}

A recent survey of aircraft accidents in the UK showed that only $10 \%$ of recent accidents could be attributed to airworthiness causes [24]. However, there are 
10 non-fatal accidents for every fatal one, and also there are many fatigue failures which do not result in serious accidents. This represents an economic problem rather than one with human dimension, because it is related to the cost of detection, repair and maintenance required in order to avoid that fatigue failures becoming fatal accidents.

In this research, we are proposing a methodology that allows calculating either the probability of finding a crack of any size or the probability of airframe failure using a probabilistic approach. Fundamentally, the proposed approach is to develop a crack size probability density function corresponding to the fixed time (cumulative damage state) of crack initiation. Then the projection of crack size distribution for a specific aircraft at the present age of the aircraft is computed by a probabilistic crack growth analysis method in which loads are known by tracking the data and the crack growth start from the initial crack distribution. Once the present crack size distribution for a specific aircraft is ascertained it is possible to compute reliability of some future flight regimes. Such calculations are done based on probabilistic crack growth analysis, in which both material properties and load are random variables. In essence, the key question is the reduction of the uncertainty of the initial crack size distribution. A Bayesian approach using two different methods, based on conjugate and Markov Chain Monte Carlo (MCMC) simulation, is developed to take into consideration the findings that are to become available from an extensive fleet inspection program.

Moreover, this research assesses the uncertainty about the empirical fatigue crack growth models. This uncertainty including the scatters in the results using 
different models, and the factors that affect the crack growth rate, justify the need for developing a tool that allows the users to manage the crack growth life prediction in aging aircraft.

\subsection{Contributions}

The specific contributions of this research are:

- The development of a joint damage-life remaining distribution showing fatigue crack size distribution at different damage levels. This distribution is applied to analyze the behavior of the fatigue crack growth under random loads and material variability.

- The development of a method for characterizing crack growth deterministic models and their uncertainties.

- Discussing options for Decision-making based on the crack growth model including uncertainties.

\subsection{Application}

Application of the methodology developed in this dissertation is in terms of aircraft type, the analysis location, the aircraft material experiencing fatigue related failures, and the selected models for analysis. The following subsections provide specific details on each of these areas.

\subsubsection{Aircraft}

In this research, we will analyze the structural part of one military aircraft. This aircraft fleet is more than 15 years old, therefore it is considered aged. 


\subsubsection{Analysis Location}

In spite of the fact that an aircraft has more that one critical location susceptible to fatigue failure, the analysis in this work was carried out in an area near the engine, which was identified as a "hot spot" by the results obtained during the inspections carried out over the screw and rivet holes. Figure 1.3 shows the layout of the area, and the location where the analysis was carried out.

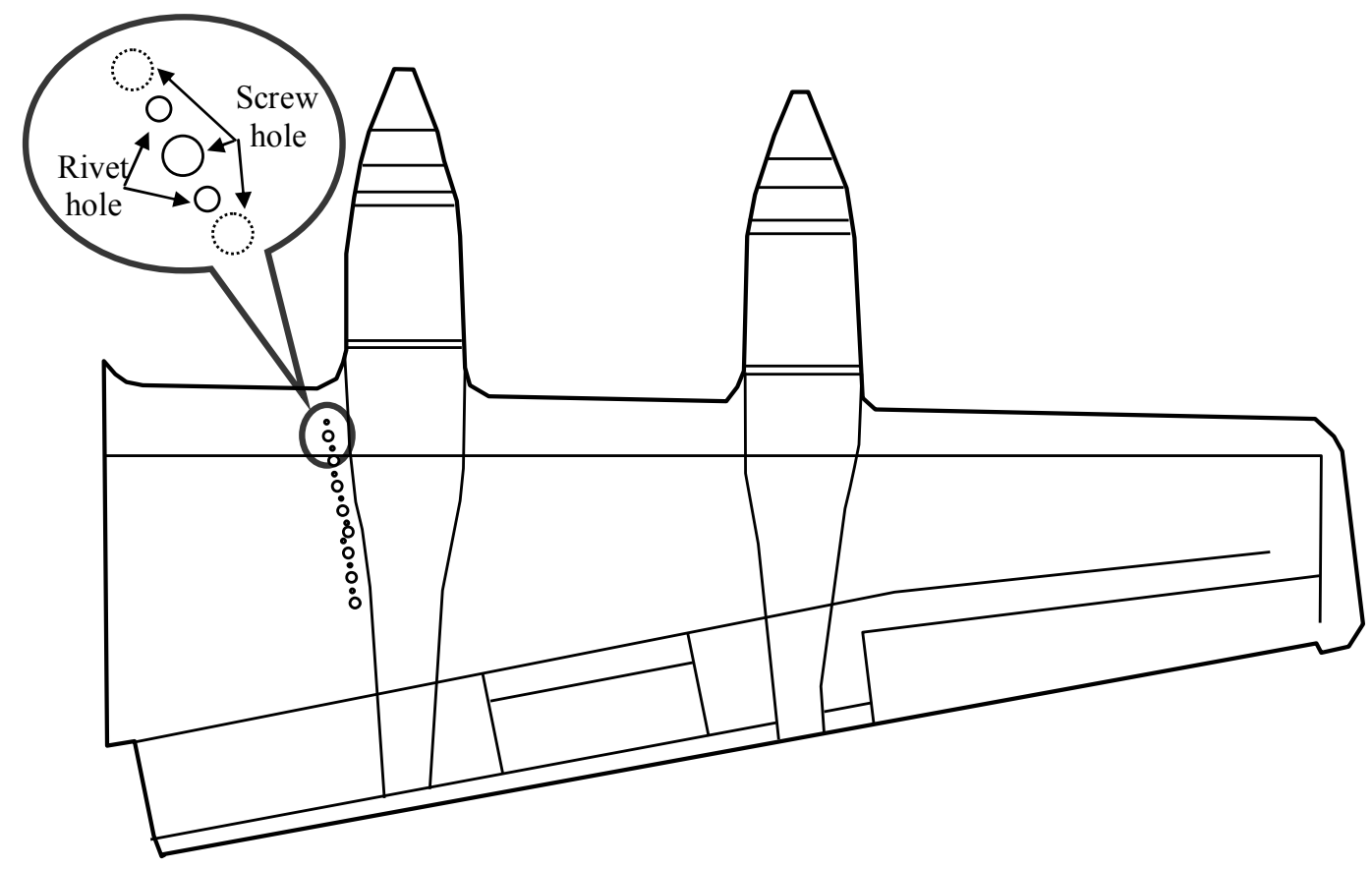

Figure 1.3 Layout of the part, and the location where the analysis was carried out.

It is important to note that the developed methodology allows the estimation of fatigue life in different locations in the aircraft taking into consideration the geometry of the metallic structure, which defines the geometry factor used in the crack growth calculation. 


\subsubsection{Materials}

The material of the structure under analysis is aluminum 7075-T6 (UNS A97075), which provides excellent strength-to-weight ratio and is one of the highest strength aluminum alloys available. Its high strength sacrifices other important properties, such as formability. It can be formed in the annealed condition followed by heat treatment to give it specific mechanical properties. Its corrosion resistance is good, due to its copper contents. This material is typically used for highly stressed parts, especially in the aircraft and space industries [25].

\subsubsection{Models}

There is no a universal fatigue crack growth model. This statement is based on the fact that a relatively large number of models are found in the literature. Most of these models were developed to account for the different factors affecting the crack growth rate. Table 1.1 shows some of these models and the numbers of experimentally determined parameters involved in the calculation. Because we are treating these parameters as uncertain, the uncertainty of the output of models is greater as the number of parameters become large. On the other hand, the models with greater number of parameters may yield more accurate results, because they are taking into account more factors that can affect the fatigue crack growth. Therefore, the life estimation should be a tradeoff between precision and the uncertainty propagation due to the number of parameters involved in the model. 
Table 1.1 Parameters of crack growth models.

\begin{tabular}{|c|c|c|c|c|c|c|}
\hline & $\begin{array}{l}\text { Paris } \\
\text { model }\end{array}$ & $\begin{array}{c}\text { Forman } \\
\text { model }\end{array}$ & $\begin{array}{l}\text { Walker } \\
\text { model }\end{array}$ & $\begin{array}{c}\text { Generalized } \\
\text { Willenborg model } \\
{[26]}\end{array}$ & $\begin{array}{c}\text { Fastran } \\
\text { model [27] }\end{array}$ & $\begin{array}{c}\text { Nasgro } \\
\text { model [26] }\end{array}$ \\
\hline \multirow{9}{*}{ 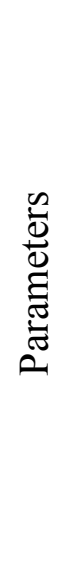 } & $m$ & $m_{1}$ & $m_{2}$ & $m_{4}$ & $m_{5}$ & $m_{6}$ \\
\hline & $C$ & $C_{1}$ & $C_{2}$ & $C_{4}$ & $C_{5}$ & $C_{6}$ \\
\hline & & $\beta$ & $\gamma$ & $\alpha$ & $q$ & $p$ \\
\hline & & & $\beta$ & $R_{\mathrm{SO}}$ & $\alpha$ & $q$ \\
\hline & & & & $\beta$ & $K_{\mathrm{IC}}$ & $\alpha$ \\
\hline & & & & & $\sigma_{0}$ & $\sigma_{0}$ \\
\hline & & & & & $\beta$ & $C_{\mathrm{th}}$ \\
\hline & & & & & & $a_{0}$ \\
\hline & & & & & & $\beta$ \\
\hline
\end{tabular}

In this research, we analyze the fatigue cracks phenomenon using three models that have been extensively used in the literature. These models are: Forman, Walker and Crack Closure. These models will be described in more details in Chapter 4.

\subsubsection{Structure}

This dissertation is structured according to the diagram in Figure 1.4. This outline is consistent with the selected method of analysis.

Chapter 2 covers the technical description of fatigue and material properties of aluminum 7075-T6 (UNS A97075).

Chapter 3 describes the data collection process. Specifically, the chapter explains how the load (stresses) for the analysis is collected, the definition of the Initial Crack Size (ICS) distribution, and how the evidence (inspections finding) data is collected. 
The fatigue crack growth models are described in Chapter 4. This includes the Forman, Walker and Closure crack growth models, and the fatigue life estimation and uncertainty propagation from the input variables using a Monte Carlo simulation.

Chapter 5 describes the probabilistic fatigue life model used to analyze the data and explains the probabilistic model parameter estimation of the fatigue life model using the Maximum Likelihood Estimator approach. A comparison of the values estimated is carried out using the MCMC and Genetic Algorithm methods.

Chapter 6 will cover the Bayesian updating process of the ICS distribution. Two different approaches are used, the first one based on the Conjugate Lognormal, and the second based on the Monte Carlo Markov Chain (MCMC).

Chapter 7 describes the analysis of the results and the strategies to follow in order to deal with the output from the three models used in the analysis.

Finally, Chapter 8 shows the conclusions and recommendations of the research. 


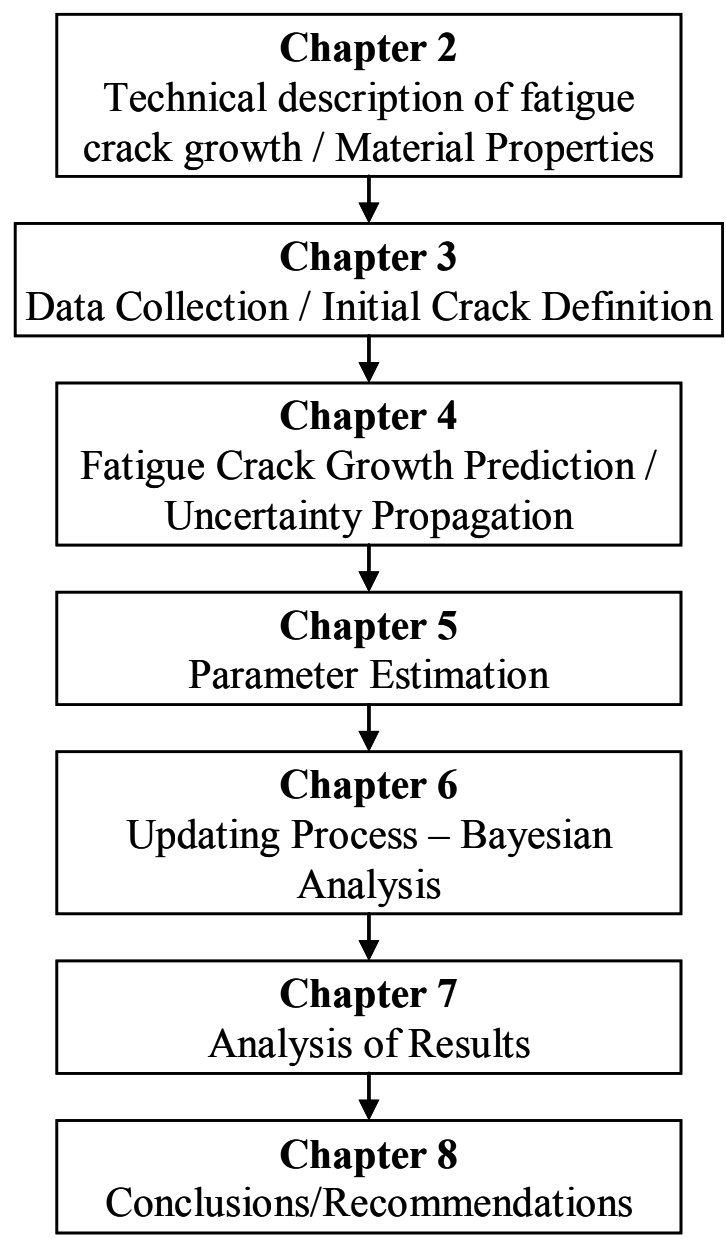

Figure 1.4 Structure of the dissertation. 


\section{Chapter 2 Technical Description of Fatigue Crack \\ Growth / Material Properties}

\subsection{Fatigue Crack Growth Phenomenon}

Fatigue is one of the most important phenomenon to take into account in engineering systems. Fatigue is an important phenomenon, because it is related to the economy and the safety [28]. Economy, because the cost of high reliability structures are high and early removal from service is not economical, and safety, because the consequences of failure due to fatigue can be catastrophic.

Appreciation of the fatigue problem is peculiar from two different points of view. For instance, from the perspective of designers, their interest is to produce a structure with high fatigue resistance and low cost, therefore, they have to account for aspects such as cyclic stresses, material properties, surface roughness, etc. On the other hand, from the users' point of view, their interest is to use the items in a safe manner; therefore, they have to account for aspects such as the utilization of the structure, environment that may have been overlooked by the designer, etc.

For the users, it is important to have knowledge about the areas susceptible to crack, and how fast the crack grows in order to manage the life of the structure, therefore, maintenance, inspection and non-destructives techniques play a key role in the fatigue phenomenon. Moreover, dealing with fatigue depends on the type of structure under analysis. Schijve lists three examples (see Table 2.1) to illustrate this concept [28]: 
Table 2.1 Typical cases of fatigue problems.

\begin{tabular}{|c|c|}
\hline Structure & \\
\hline Automotive engine & $\begin{array}{l}\text { - Cracks should not occur. } \\
\text { - "Infinite" life is a requirement. } \\
\text { - Crack growth is not of interest. } \\
\text { - The key factor is to design and to } \\
\text { produce the structure free of crack } \\
\text { nucleation. }\end{array}$ \\
\hline Nuclear pressure vessel & $\begin{array}{l}\text { - Crack growth should be considered, } \\
\text { but must be very slow. } \\
\text { - Crack growth is allowable. } \\
\text { - Crack nucleation is of little interest. } \\
\text { - Initial flaws and defects in a welded } \\
\text { steel structure have to be expected. }\end{array}$ \\
\hline Aircraft & $\begin{array}{l}\text { - Crack nucleation and crack growth } \\
\text { are significant. } \\
\text { - "Finite" life has to be accepted. }\end{array}$ \\
\hline
\end{tabular}

Figure 2.1 illustrates schematic curves for both finite and infinite life for different cases of crack nucleation and crack growth. In the cases starting from polished surfaces (no defects and inclusions), it is clear that most of the fatigue life is spent in the micro crack region. The error of ignoring the remaining life is small. In these cases, it is considered that the fatigue damaging process is largely occurring in a very small volume of the material. On the other hand, starting from defects, the total fatigue life is spent in the macro crack region, and bulk properties of material have to be considered. 


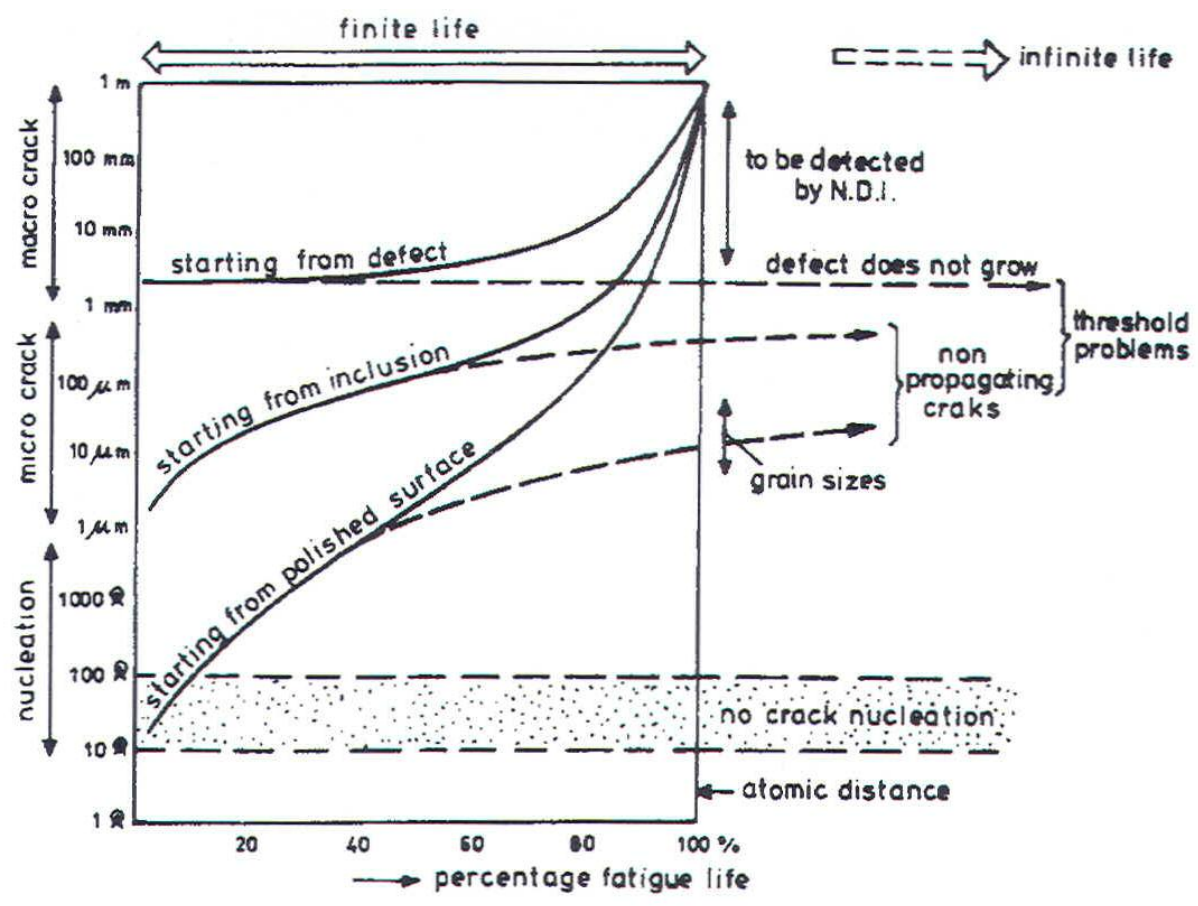

Figure 2.1 Schematic curves for both finite and infinite life [28].

In this research, we will limit the scope by considering the prediction of fatigue properties for an aircraft structure starting from a defect under variable cyclic loading. The fatigue life under cyclic loading consists of two phases, crack nucleation (crack initiation) followed by crack growth as is represented in the Figure 2.2. A problem involved in this definition is how to determine the transition between nucleation period and crack growth period (i.e., existence of a macro crack). According to the literature [29], there are several definitions trying to set this transition,

1. A macro crack is one that is large enough to be seen by the naked eyes. 
2. A crack is macro crack if it had sufficient depth (or length) to be sure that local condition, responsible for crack nucleation does not longer affect crack growth.

3. A crack is a macro crack as soon as fracture mechanics are applicable. In other words, a crack is a macro crack as soon as the stress intensity factor, $K$ has a real meaning for describing its growth.

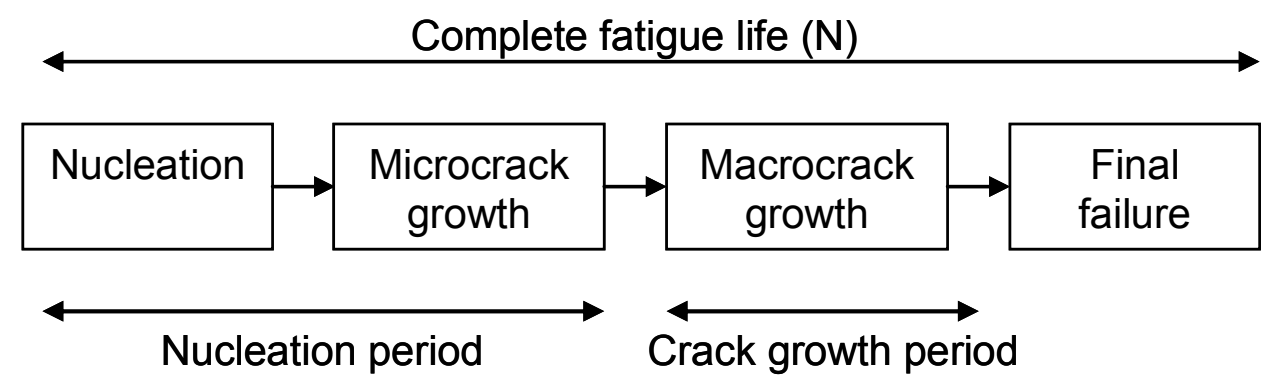

Figure 2.2 Fatigue life [28].

Fatigue cracks generally start at the surface of the material. Among the factors that may contribute are: high stress levels, surface roughness, and environmental effects. All these aspects will promote crack nucleation at the surface. In addition, material structure and residual stresses may contribute. However, they are not necessarily unfavorable for fatigue (e.g. shot peening) [28].

At stress levels near the fatigue limit, it may happen that only one crack has been nucleated. Typically there is a weakest link in a material which may have as many as 1000 grains per $\mathrm{mm}^{2}$. This weak link is a highly local phenomenon in the nucleation period, while at higher stress level several weak links are ready to produce a crack. Therefore, the fatigue phenomenon is still a local process for a long time, i.e. microcracks are present in a rather small volume of the material only. 
Figure 2.3 shows the impact of surface roughness. The quality of the surface finish has a large effect on the nucleation period, while the effect on the crack growth period is negligible. Therefore, macrocrack growth is depending on bulk material properties, since it is no longer a localized phenomenon.

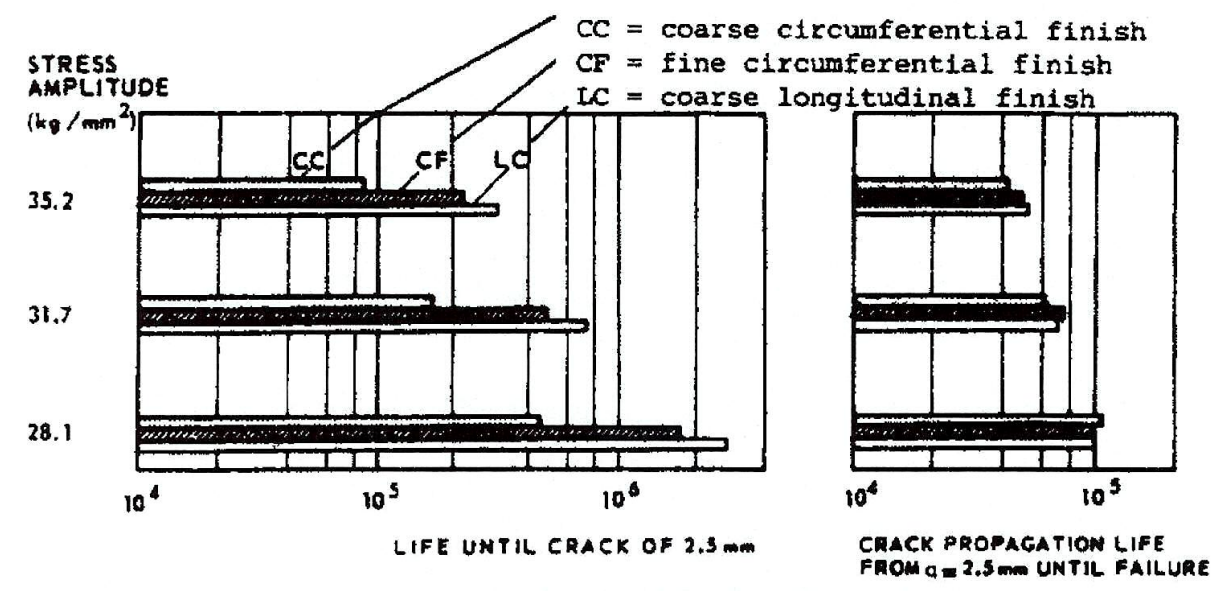

Figure 2.3 Effect of surface finish on the pre-crack life and the crack propagation life of unnotched rotating beam specimens of $0.2 \% \mathrm{C}$ steel (SAE 1020) [28].

It is important to note that crack nucleation is not always followed by crack propagation due to compressive stresses. The environment affects crack propagation. The crack rate is slower under inert environment, and faster under aggressive environments, such as salt water.

To overcome the above factors a practical approach is to correlate crack growth rates under similar conditions, which imply the same loading on the crack tip area, described by the stress intensity factor, and the same environment surrounding the crack tip.

Figure 2.4 illustrates the approach followed in this research, which is based on the concept of fracture mechanic and linear elastic fracture mechanics (LEFM), where 
the stress intensity factor, $K$, and the stress intensity factor range, $\Delta K$, are the base of the calculations.

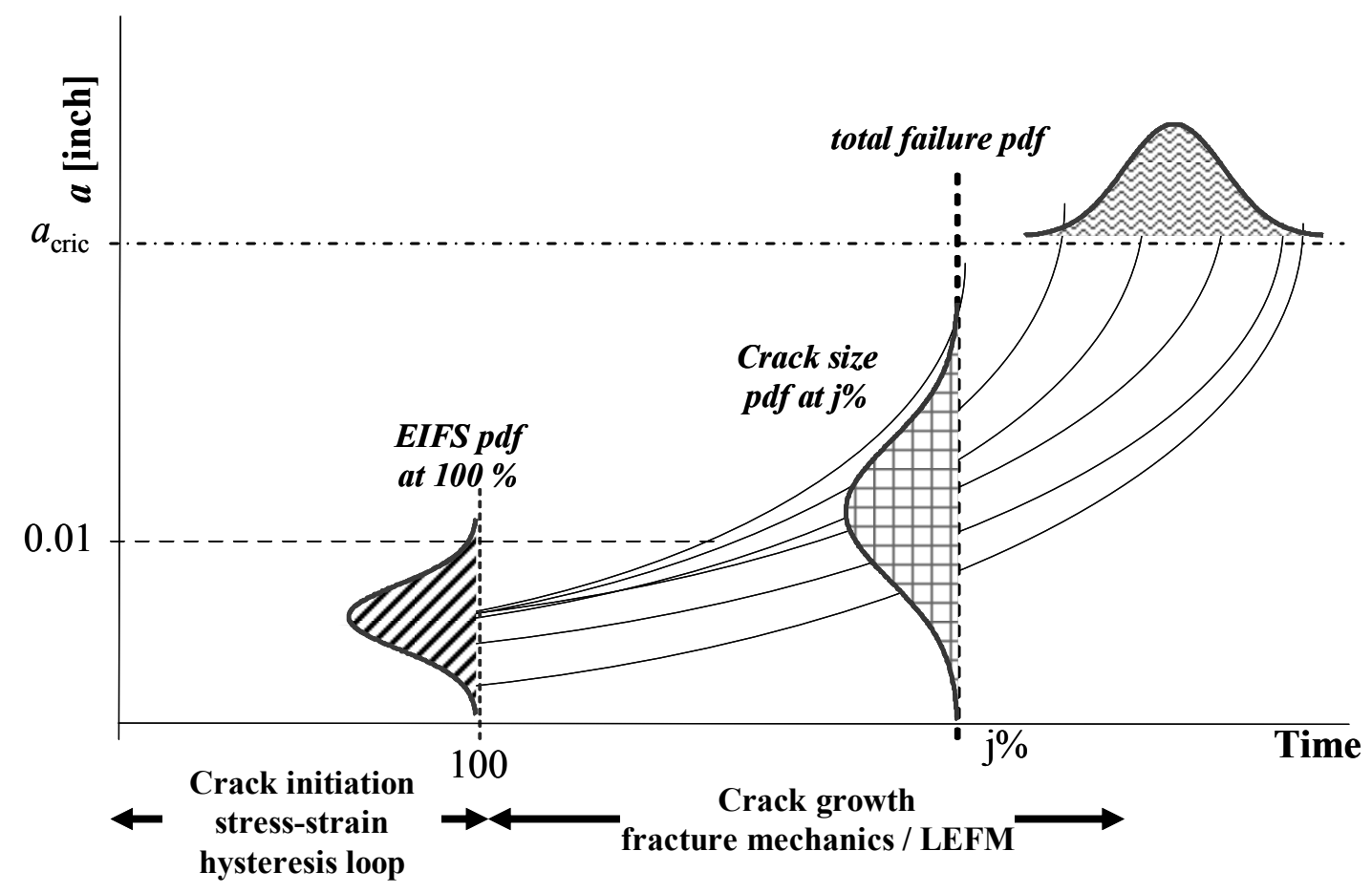

Figure 2.4 Approach followed in the research.

\subsection{Linear Elastic Fracture Mechanics}

The fracture mechanics concept has been used extensively in the aerospace, nuclear and ship industries. It uses the stress intensity factor, the strain energy release rate, and the J-integral. Linear Elastic Fracture Mechanics (LEFM) assumes that the material is isotropic and linearly elastic. Based on this assumption, the stress field near the crack tip is calculated using the theory of elasticity. When the stresses near the crack tip exceed the material fracture toughness, the crack will grow [30]. In 
LEFM, most formulas are derived for either plane stresses or plane strains, associated with the three basic modes of loadings on a cracked body.

In the following sections, we will briefly discuss: stress intensity factor, plastic zone near the crack tip, and the fracture toughness.

\subsubsection{Stress Intensity Factor}

The stress intensity factor can be defined from the stress analysis of cracks. There are three modes in which a crack can extend. Mode I, which is the most common in the fatigue field, is an opening or tensile mode, and Modes II and III are the sliding and tearing modes, respectively. In order to develop the stress intensity factor derivation, it is assumed that there is a crack in a linear elastic isotropic block subjected to Mode I loading. Figure 2.5 shows the stress in the vicinity of this crack tip with coordinates $r$ and $\theta$.

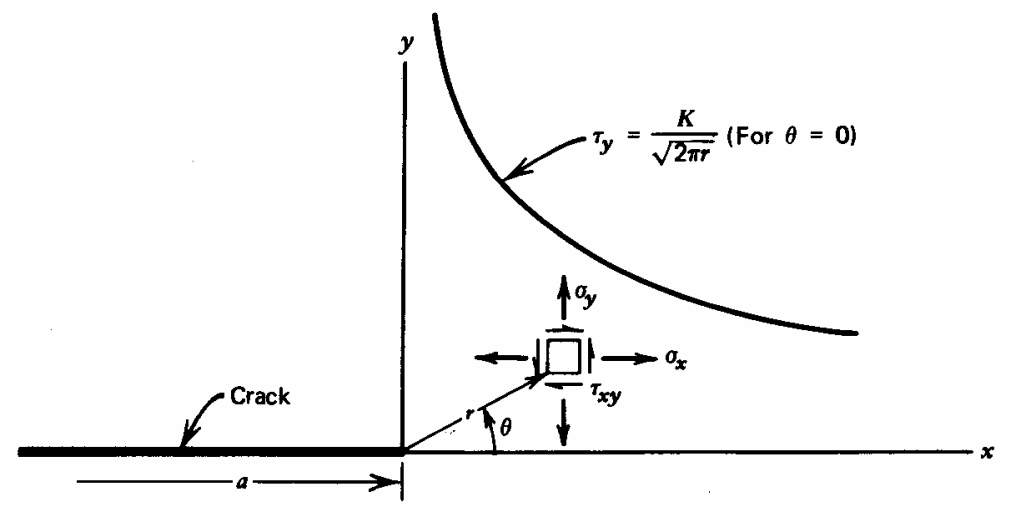

Figure 2.5 Elastic stresses near the crack tip $(\mathrm{r} / \mathrm{a}<<1)[2]$.

The stresses at any point near the crack tip can be derived through the use of mathematical theory of linear elasticity and the Westergaard stress function in 
complex form [2]. It is important to notice that by definition, the normal and shear stresses in the $\mathrm{z}$ direction are zero for plane stress, while the normal and shear strains involving the $\mathrm{z}$ direction are zero for plane strain. In polar coordinates, the equations around the crack tip are defined by,

$$
\left.\begin{array}{l}
\sigma_{\mathrm{y}}=\frac{K}{\sqrt{2 \pi r}} \cos \frac{\theta}{2}\left[1+\sin \frac{\theta}{2} \sin 3 \frac{\theta}{2}\right] \\
\sigma_{\mathrm{x}}=\frac{K}{\sqrt{2 \pi r}} \cos \frac{\theta}{2}\left[1-\sin \frac{\theta}{2} \sin 3 \frac{\theta}{2}\right] \\
\tau_{\mathrm{xy}}=\frac{K}{\sqrt{2 \pi r}} \cos \frac{\theta}{2} \sin \frac{\theta}{2} \cos 3 \frac{\theta}{2} \\
\sigma_{\mathrm{z}}=\sigma_{\mathrm{xz}}=\sigma_{\mathrm{yz}}=0 \text { for plane stress } \\
\sigma_{\mathrm{z}}=\mu\left[\sigma_{\mathrm{x}}+\sigma_{\mathrm{y}}\right] \\
\tau_{\mathrm{xz}}=\tau_{\mathrm{yz}}=0
\end{array}\right\} \text { for plane strain }
$$

where $K$ is the stress intensity factor, and the polar coordinates $r$ and $\theta$. From this calculation, it is possible to prove that elastic normal and elastic shear stresses in the vicinity of the crack tip depend on $r, \theta$, and $K$. Because the magnitudes of these stresses at a given point are dependent entirely on $K$, then $K$ is called stress intensity factor. When $K$ is used without a mode subscript, it refers to mode I.

The elastic stress distribution in the y direction for $\theta=0$ is shown in Figure 2.5. It can be seen that the stress at the crack tip approaches infinity as $r$ approaches zero. Therefore, a stress singularity exists at $r=0$, and the elastic solution must be modified to account for crack tip plasticity. However, if the plastic zone size $r_{y}$ at the crack tip is small in comparison to local geometry $\left(r_{\mathrm{y}} /\right.$ thickness and $\left.r_{\mathrm{y}} / a \leq 0.1\right)$ little or not modifications to $K$ is needed [2]. This last statement imposes an important restriction to the use of LEFM, which state that the plastic zone size at the crack tip 
must be small relative to the geometrical dimensions of the structure. However, a definite limiting condition for LEFM is that nominal stresses in the crack plane must be less than the yield strength.

Finally, stress intensity factor for different geometries, configurations, and loadings is given by,

$$
K=\beta S \sqrt{\pi a}
$$

where $S$ is the far field stress, $a$ is the crack size and $\beta$ is the geometry factor. The geometry factor is a dimensionless parameter that depends on the crack size and width of the piece. The value of the $\beta$ factor highly depends on the geometry of the structure. For example, for a given geometry Figures 2.6 and 2.7 [31] show $\beta$ along with its associated uncertainties.

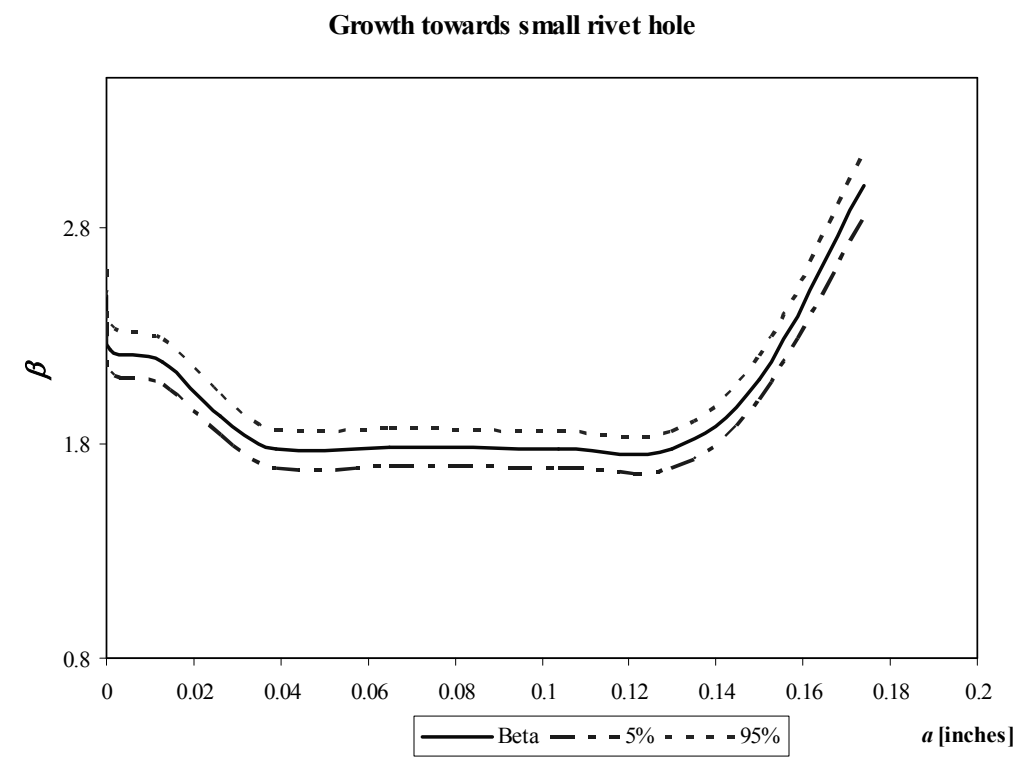

Figure 2.6 $\beta$ factor for the growth towards small rivet holes. 


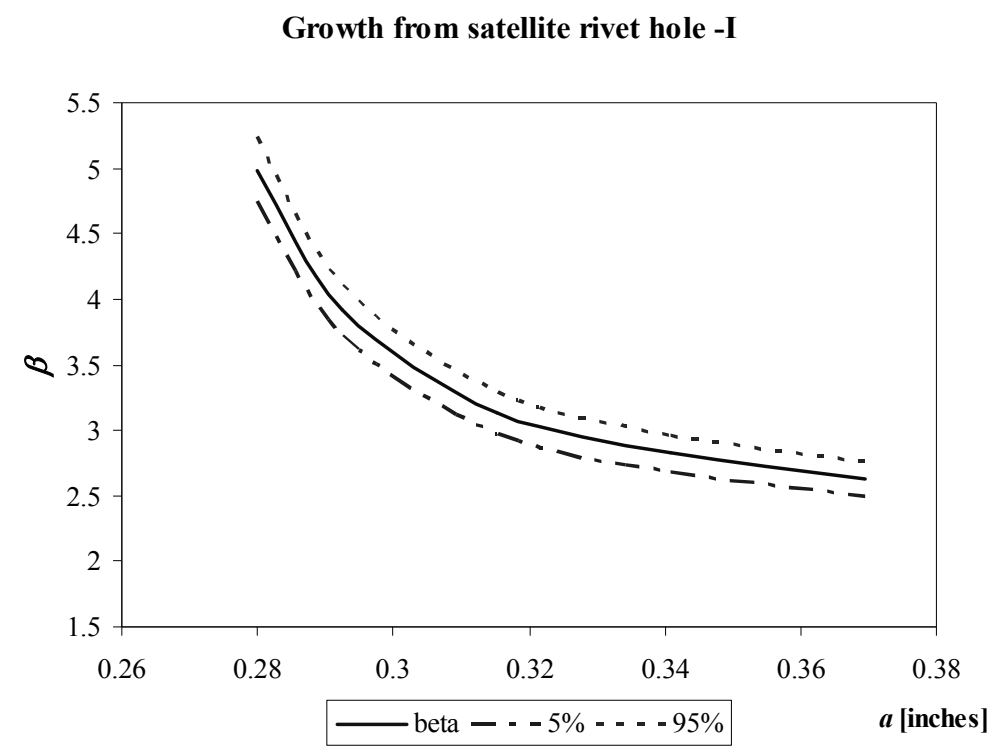

Figure 2.7 $\beta$ factor for the growth from satellite rivet holes.

\subsubsection{Crack tip Plastic Zone Size}

The local plasticity at the crack tip is known to control both the crack growth and fracture. From equation 2.1, it is possible to calculate the plastic zone size near the crack tip as a function of the stress intensity factor and the yield strength. Figure 2.8 shows the resultant monotonic plastic zone shape for mode I, using the von Mises criterion [2]. Figure 2.8 also illustrates that for plane stress conditions, the plastic zone is larger than the plane strain condition, because the value of $\sigma_{z}$ have different values for plane stress and plane strain, which decreases the magnitude of two of the three principal shear stresses. 


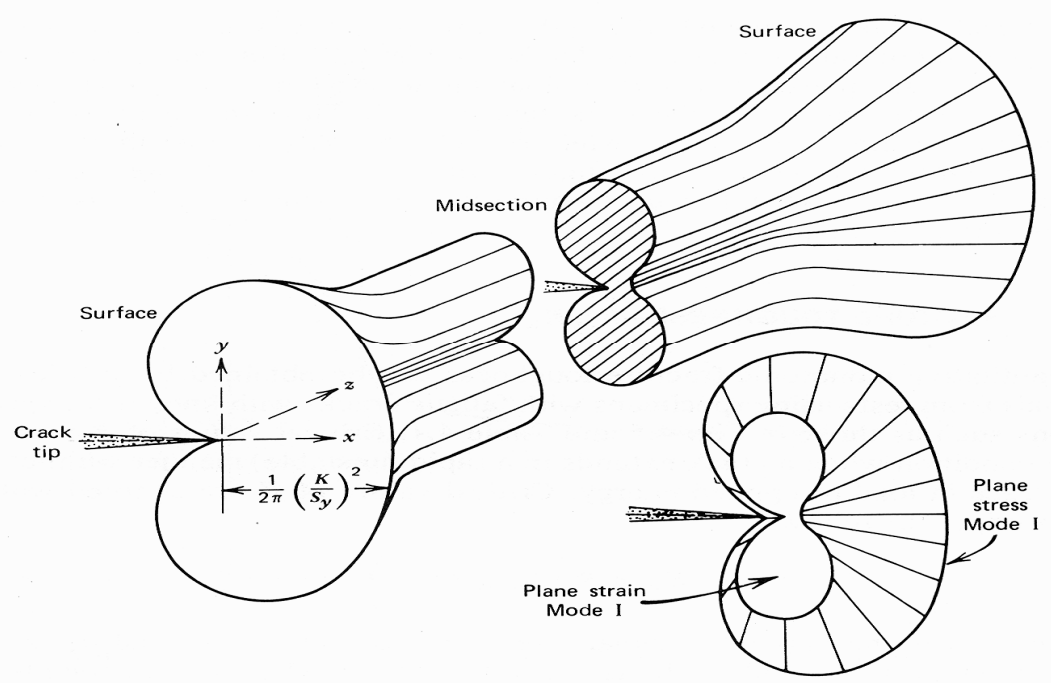

Figure 2.8 Plastic zone size at the tip of a through thickness crack [2].

The plastic zone is proportional to the square of the ratio of the stress intensity factor to the yield strength, and due to the relaxation of the stress field in the plastic zone, the actual plane stress plastic zone size is approximately twice this value. Moreover, the plane strain plastic zone size in the plane of the crack is generally taken as one-third the plane stress value. Under monotonic loading, the plane stress plastic zone, $2 r_{\mathrm{y}}$, at the crack tip is given by [2],

$$
2 r_{\mathrm{y}}=2\left[\frac{1}{2 \pi}\left(\frac{K}{S_{\mathrm{y}}}\right)^{2}\right]=\frac{1}{\pi}\left(\frac{K}{S_{\mathrm{y}}}\right)^{2}
$$

and for plane strain,

$$
2 r_{\mathrm{y}} \cong \frac{1}{3 \pi}\left(\frac{K}{S_{\mathrm{y}}}\right)^{2}
$$

In the literature there are additional models for plastic zone size and shape that have been widely used [2]. 


\subsubsection{Fracture Toughness}

Fracture toughness is defined as the resistance of a material or structure from a grown crack. This definition can be mathematically expressed by the following expression:

$$
K_{\mathrm{C}}=\beta S_{\mathrm{C}} \sqrt{\pi a_{\mathrm{c}}}
$$

where $K_{\mathrm{C}}$ is the fracture toughness, $S_{\mathrm{C}}$ is the applied nominal stress at crack instability and $a_{\mathrm{c}}$ is the crack length at instability. Fracture toughness depends on the material, temperature, strain rate, environment, thickness, and to a lesser extent, crack length [2]. Figure 2.9 shows the relationship between $K_{\mathrm{C}}$ and thickness. It is seen that thin parts have a high value of $K_{\mathrm{C}}$ accompanied by appreciable slant fracture (plane stress). As the thickness increase, the $K_{\mathrm{C}}$ and percentage of slant fracture decreases (mixed mode). For thick parts, essentially the entire fracture surface is flat and $K_{\mathrm{C}}$ approaches an asymptotic minimum value (Plane strain). The minimum value of fracture toughness is known as plane strain fracture toughness, $K_{\mathrm{IC}}$. Further increase in thickness does not the decrease $K_{\mathrm{IC}}$ value. The subscript I is used, because the fact that these fractures occur almost entirely by mode I crack opening. 


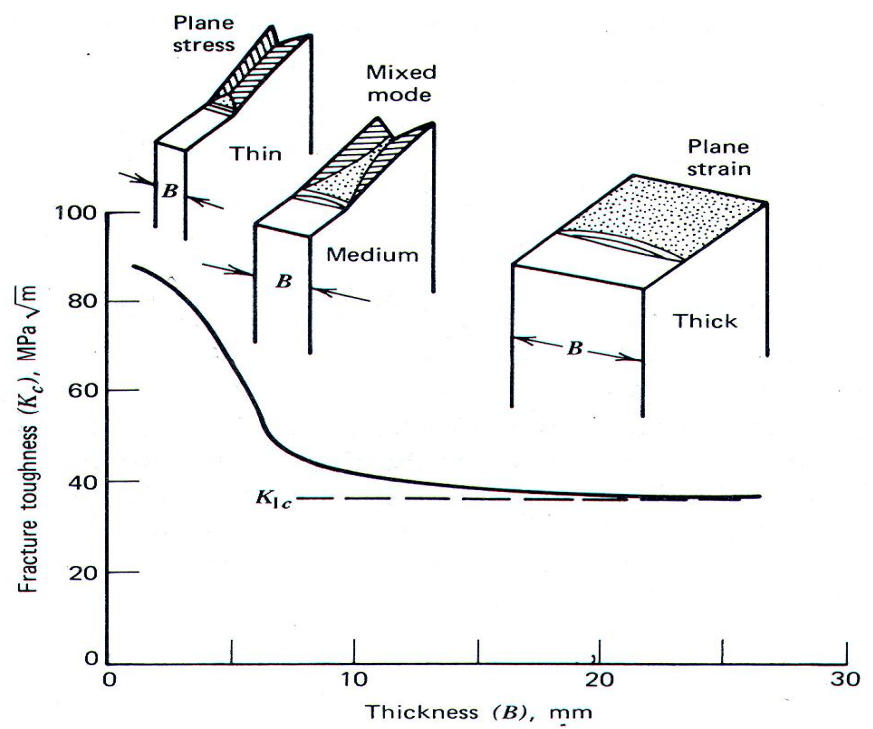

Figure 2.9 Effect of specimen thickness on fracture toughness [2].

If the fracture toughness is known for a given material and thickness, and the stress intensity factor is known for a given component and loading, it is possible to estimate a design criterion to prevent fracture. For the plane strain fracture toughness $K_{\text {IC }}$ to be a valid failure criterion, plane strain conditions must exist at the crack tip. This means, the material must be thick enough to ensure plane strain conditions. It has been estimated empirically that for plane strain conditions the minimum material thickness $B$ must be [32],

$$
B \geq 2.5\left(\frac{K_{I C}}{S_{y}}\right)^{2}
$$

Using the LEFM concept, one can translate the information from the fatigue crack length and applied cycles to fatigue prediction. Figure 2.10 shows an example of three crack length versus applied cycle curves for three identical test specimens, 
which are subjected to different repeated stress levels. All the specimens have the same initial crack size, and the minimum stress was zero. It is clear that the higher the stress level, the shorter the fatigue life. Consequently, we can say that total life to fracture depend on the initial crack size, the stress magnitude, and the final fracture resistance of the material. However, this information is not applicable to fatigue prediction except under the exact same conditions used in obtaining the data [2]. Therefore, through the LEFM concept, it is possible to obtain a sigmoidal curve, where the axes are the crack growth rate, $\mathrm{d} a / \mathrm{d} N$, and the applied stress intensity factor range, $\Delta K$. The stress ranges, $\Delta S$, and crack size, $a$, are included in $\Delta K$.

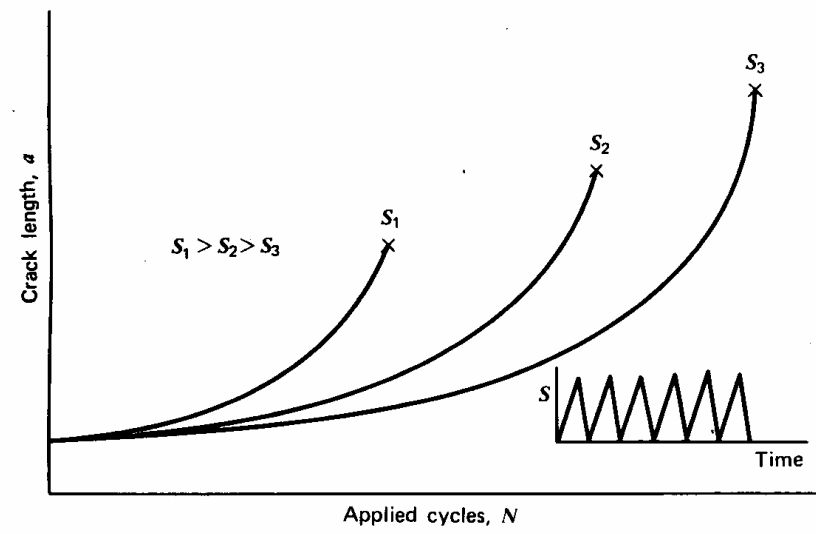

Figure 2.10 Fatigue crack length vs. applied cycles. Fracture is indicated by X [2].

Using the proper stress intensity factor for a given component and crack, integration of the sigmoidal sharpe curve can provide fatigue crack growth life for components subjected to different stress levels and different initial crack sizes. The following section will cover the sigmoidal shape $\mathrm{d} a / \mathrm{d} N$ curve. The initial crack size and the stress load will be covered in Chapter 3. 


\subsection{Sigmoidal Shape $\mathrm{d} a / \mathrm{d} N$ Curve}

The stress intensity factor was introduced in the early 1960s as the correlation between the crack growth rate, $\mathrm{d} a / \mathrm{d} N$, and the stress intensity factor range, $\Delta K$. Paris et al. published the results in terms of $\mathrm{d} a / \mathrm{d} N$ as a function of $\Delta K$ using a $\log -\log$ scale, where it is possible to see a linear relationship between $\log (\mathrm{d} a / \mathrm{d} N)$ and $\log (\Delta K)$. After the publication of the Paris et al. results, several crack growth test were carried out by other authors, obtaining the same trend, which led to the well-known Paris equation [14],

$$
\frac{\mathrm{d} a}{\mathrm{~d} N}=C \Delta K^{m}
$$

where $C$ and $m$ are experimentally estimated constants. At the same time, crack growth is subjected to physical laws [33]. In general terms, there is a crack driving force, which is associated with the stress intensity factor. The material response $(\mathrm{d} a / \mathrm{d} N)$ is characterized in equation 2.7 , but the experimental constant $C$ and $m$ are not easily associated with physical properties of the material. However, the crack growth rate obtained is representing the crack growth resistance of the material.

Moreover, the results of crack growth test indicate systematic deviations of Equation 2.7 at relatively high and low stress intensity factor values, which led to the definition of three regions in the $\mathrm{d} a / \mathrm{d} N$ curve, see Figure 2.11. 


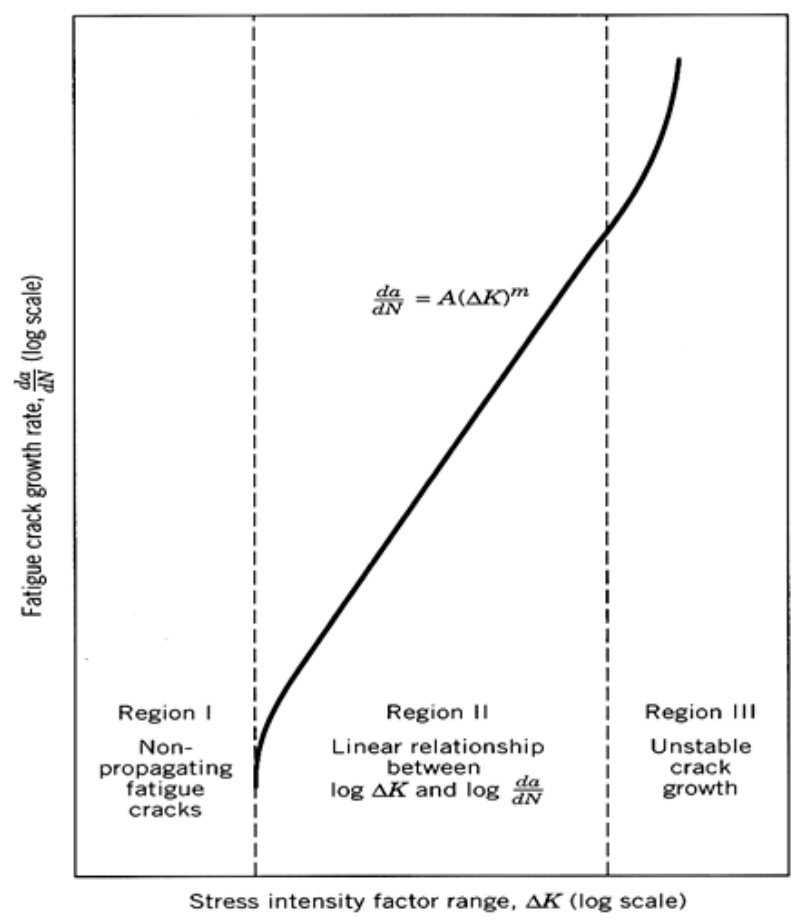

Figure 2.11 Fatigue crack growth rate behavior [34].

Region I, depict low stress intensities, where the crack growth is associated with a threshold value, $\Delta K_{\text {th }}$, which is in the range of 3 and $6 \mathrm{ksi}-\sqrt{\mathrm{in}}$, for aluminum alloys [35]. Below this value, crack growth occurs at a rate too slow to measure. For example, the smallest measured rates are larger than approximately $10^{-8} \mathrm{in} /$ cycle, which corresponds to the spacing between atoms in most metals. Due to the sensitivity of $\Delta K_{\text {th }}$ to the environment and load history, it is recommended to carry out the fatigue tests under conditions that simulate the actual service conditions [36]. Region II also known as Paris regime, encompasses data where the rate of crack growth changes roughly linearly with a change in stress intensity fluctuation, i.e., exhibits a linear variation of $\log (\mathrm{d} a / \mathrm{d} N)$ with $\log (\Delta K)$, and in region III the crack 
growth rates are very high and little fatigue crack growth life is involved [2]. This region is controlled primarily by the fracture toughness $K_{\mathrm{C}}\left(K_{\mathrm{IC}}\right)$.

Table 2.2 summarizes the characteristics of crack growth in the three regions of stable fatigue fracture.

Table 2.2 Characteristics of the three regimes of fatigue crack growth [2].

\begin{tabular}{llll}
\hline \multicolumn{1}{c}{ Region } & \multicolumn{1}{c}{ I } & \multicolumn{1}{c}{ II } & \multicolumn{1}{c}{ III } \\
\hline Terminology & $\begin{array}{l}\text { Slow-growth rate } \\
\text { (near threshold) }\end{array}$ & $\begin{array}{l}\text { Mid-growth rate } \\
\text { (Paris regime) }\end{array}$ & High-growth rate \\
\hline $\begin{array}{l}\text { Microscopic failure } \\
\text { mode }\end{array}$ & Stage I, single shear & $\begin{array}{l}\text { Stage II (striations) } \\
\text { and duplex slip }\end{array}$ & Additional static \\
\hline $\begin{array}{l}\text { Fracture surface } \\
\text { features }\end{array}$ & Faceted or serrated & Planar, with ripples & $\begin{array}{l}\text { Additional } \\
\text { cleavage or } \\
\text { microvoid } \\
\text { coalescence }\end{array}$ \\
\hline Crack closure levels & High & Low & --- \\
\hline $\begin{array}{l}\text { Microstructural } \\
\text { effects }\end{array}$ & Large & Small & Large \\
\hline Load ratio stress & Large & Small & Large \\
\hline $\begin{array}{l}\text { Environmental } \\
\text { effects }\end{array}$ & Large & Small \\
$\begin{array}{l}* * * \\
\text { Large influence on crack growth for certain combinations of environment, load ratio }\end{array}$ & &
\end{tabular}

It has been observed that Equation 2.7 requires adjustments to account for the commonly observed effects of ratio (mean) stresses, and the nonlinear effects observed when a complex time history of load is employed [37]. These two effects will be covered in the following sections. 


\subsection{Effect of Stress Ratio Stress on fatigue Crack Growth}

Crack growth tests are commonly carried out at zero-to tension loading stress ratio, $R=0$. However, it is clear from this test that the correlation $\mathrm{d} a / \mathrm{d} N$ and $\Delta K$ depends on the stress ratio. An increased mean stress for a constant $\Delta S$ should give a faster crack growth while the $R$ value is also increased, which is illustrated in Figure 2.12. The effect is generally more pronounced for more brittle materials. In contrast, mild steel and other relatively low-strength, highly ductile, structural metals exhibit only a weak $R$ effect in the intermediate region of the $\mathrm{d} a / \mathrm{d} N$ vs. $\Delta K$ curve [38]. Forman's equation and Walker's equation, two relationships mostly used to compensate for ratio effect, will be explained in more details in Chapter 4.

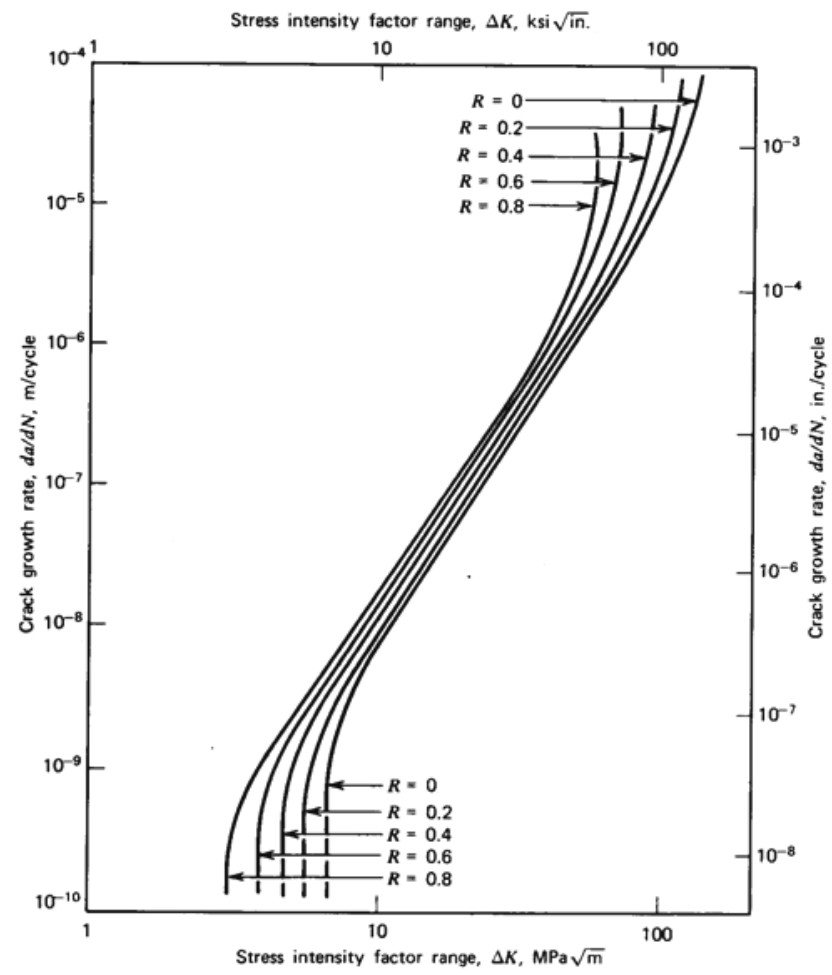

Figure 2.12. Schematic mean stress influence on fatigue crack growth rates [2]. 


\subsection{Effects of complex time history loads on fatigue crack growth}

Under variable loading the increment of fatigue crack growth also depend on the preceding cyclic loading history, which is known as load interaction. According to Schijve [29], the crack growth increment in a certain load cycle will be a function of:

1. the crack geometry being present before the cycle started,

2. the condition of the crack tip material, and

3. the magnitude of the load cycle.

Several tests have been performed on materials to have a better understanding of the interaction effects. Some of the observations as result of tests with overloads loading as discussed below:

a. Positive overloads introduce significant crack growth delays. In general, longer delays are obtained by: (a) increasing the magnitude of the overload, (b) repeating the overload during the crack propagation life, and (c) application of blocks of overloads instead of single overload [29,39].

b. The rate of fatigue crack growth depends strongly on the order in which tensile and compressive overloads are applied [1].

c. Overload sequence effects are likely to be important where high overloads occur predominantly in one direction [38].

d. Negative overloads have a relatively small detrimental effect on crack growth. However, a negative overload added immediately after positive overloads can significantly reduce the crack growth delay of the latter one $[29,38,39]$. 
e. Delays clearly depend on the ductility of the material [29].

Figure 2.13 shows typical effects of tension-compression sequence effects on variable amplitude fatigue fracture. Case I, crack growth during constant amplitude fatigue. Case II, crack growth during tensile-compressive overload sequences, and case III, crack growth during variable amplitude loading involving single tensile overloads.

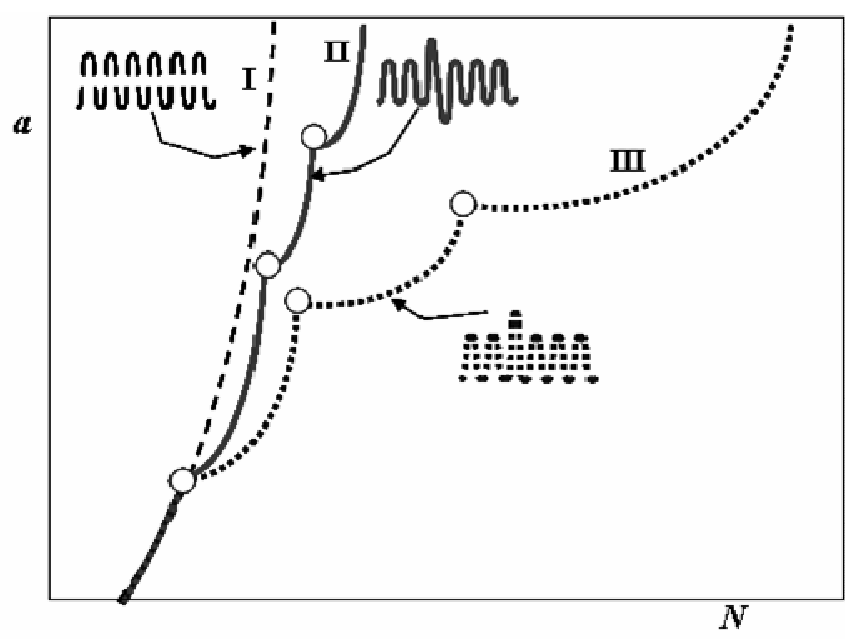

Figure 2.13 A schematic illustration of transient crack growth during constant and variable amplitude [1].

An example of crack propagation under variable amplitude loading for a 2024-T3 aluminum alloy specimen is shown in Figure 2.14. The specimen containing a center crack was subjected to a simulated flight loading of a civil transport aircraft, where the highest stress in the time history was $140 \mathrm{MPa}$. In order to simulate the unloading of a point on the lower surface of an aircraft wing, the stress was at some points reduced to zero for each flight. 


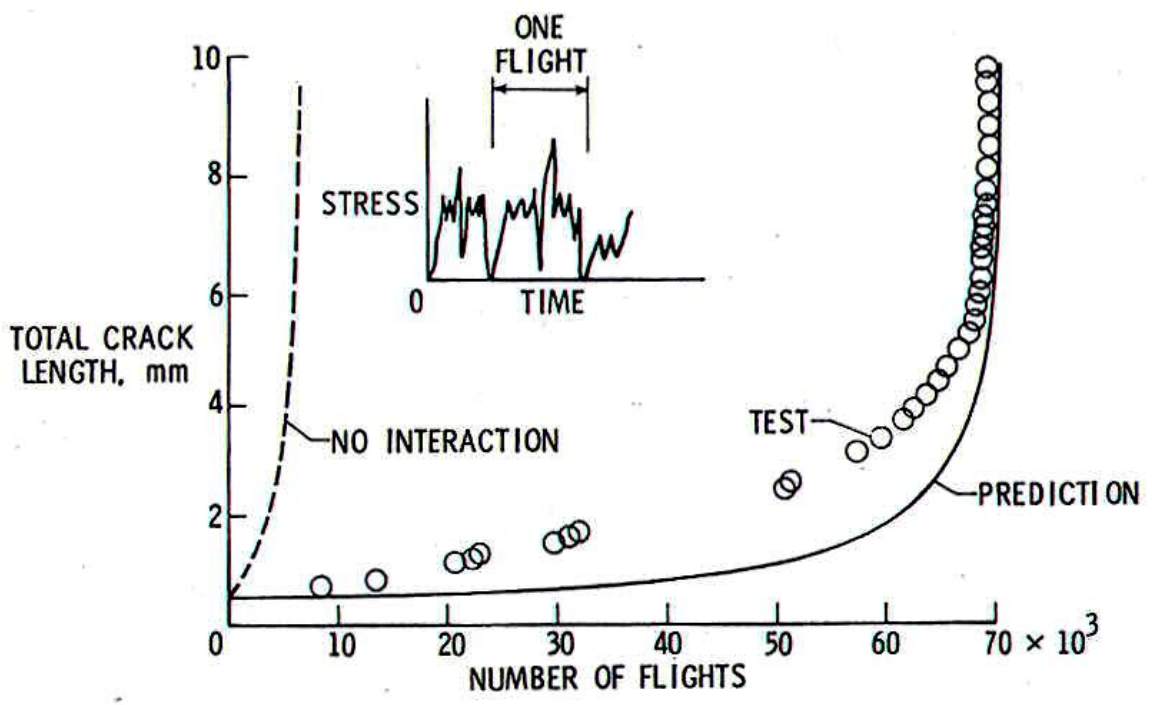

Figure 2.14 Crack propagation under simulated flight loading, 2024-T3 alum [37].

Figure 2.14 shows that the specimen survived about 70,000 simulated flights instead of the few thousand flights expected (less than 10,000), if no load iteration effects were considered. The prediction of crack growth was based on crack closure [37].

Originally, crack growth delay was based on residual stresses in the crack tip zone. In the early 1970s, Elber argued that such plasticity induced crack closure can also account for the transient retardation phenomena due to overloads [1,29].

As it is indicated in Section 2.6, the materials properties also play an important role in the crack growth phenomenon. The following section will describe the material properties that are considered in this research for fatigue prediction. 


\subsection{Material Properties}

The properties of material are those characteristics that help modify and distinguish one material from another. Taken as a whole, these qualities define a material. All properties are observable and most can be measured quantitatively with some uncertainty. Properties are classified into two main groups, chemical and physical properties. Chemical properties are associated with the transformation of one material into another. For example, iron rusts when it combines with oxygen to produce an iron oxide through a chemical reaction. Physical properties involve no change in the composition of the material. Density, strength, and hardness are examples of such properties. Physical properties are, in turn, arbitrarily subdivided into many categories. These subdivisions bear names such as mechanical, metallurgical, fabrication, general, magnetic, electrical, thermal, optical, thermonuclear, and electro-optical. Regardless of the name, physical properties result from the response of the materials to some environmental variable, such as a mechanical force, a temperature change, or an electromagnetic field [35]. For purposes of this research, only mechanical properties will be taken into account for the analysis.

\subsubsection{Mechanical Properties}

Mechanical properties are defined as a measure of a material's ability to carry or resist mechanical forces or stresses. Three tests are carried out to obtain the useful information for most applications: the tensile, hardness, and impact tests. For ductile material, the yield strength is the most important property, because can be used as a 
criterion of failure, and it can be described by the results of tensile testing. The other two tests are used to asses the durability and rigidity of the materials, respectively.

The data from the tensile tests are obtained by fracturing a specimen of material intended for a specific design project, and the fracture occurs when the material or structure separates into two or more pieces. The failure criterion used in design is different for different materials. For example, for elastomers, the failure criterion is the tear strength; for composite materials, it is tensile strength and for concrete, the criterion of failure is crushing strength or compression. As it was mentioned above, for ductile metals in particular aluminum, the design criterion is yield strength. Many factors, such as the amount of cold working and strain hardening, affect metal strength. Work done on metals directly affects metal strength, producing a range of values for its yield strength. Metal in an annealed condition would exhibit a low value, while a strain-hardened metal would be approaching its tensile strength. Depending upon the design criteria, a failure may occur prior to fracture. Beams, such as floor beams, that are designed not to deflect beyond a standard amount under normal loads may be termed a failure if deflection exceeds this stated amount. In this case, no fracture has occurred. Similarly, an aluminum desk chair that collapses and buckles is a failure even though it has not fractured. Elastic modulus, yield and ultimate strength are some of the mechanical properties used in this research. 


\subsubsection{Elastic modulus $(E)$}

The elastic modulus, also known as Young's modulus, is defined as the ratio of engineering stress $(\sigma)$ to engineering strain $(\varepsilon)$ in the linear or elastic region of the stress-strain diagram. It is also a measure of the interatomic bonding forces in a material [40]. The higher the magnitude of these bonding forces, the higher the resistance of the material to being deformed. When a material is loaded with external forces in the elastic region, the material with the highest modulus value experiences the least amount of deformation or strain, which may be either elastic or plastic deformation. If the material reverts back to its normal size and shape upon removal of the load, it is elastic deformation. If the applied force or load is removed and the material is permanently deformed, the material is said to have undergone plastic deformation.

The elastic modulus can be obtained graphically by measuring the tangent of the slope angle in the elastic region of the stress-strain curve.

\subsubsection{Yield strength $\left(S_{y}\right)$}

The yield strength is defined as the strain corresponding to the elastic limit, and it is the lowest stress at which plastic deformation occurs. For most design purposes, the yield strength is assumed to be the same in tension as in compression [40]. Strain is measured at various points in the engineering stress-strain diagram. The strain corresponding to the elastic limit is called the yield point strain $\left(\varepsilon_{y p}\right)$. The elastic limit replaces the yield point in those metals that do not show a yield point on the stress-strain diagram. Several face-centered cubic materials, for example, such as copper and aluminum, do not have a well-defined yield point. The stress at the yield 
point strain is the yield strength $\left(\mathrm{S}_{\mathrm{ys}}\right)$. The strain corresponding to the tensile strength is called uniform strain $\left(\varepsilon_{u}\right)$, because the strain to this point is uniformly distributed throughout the specimen or gauge cross section. The engineering strain at the fracture point $\left(\varepsilon_{f}\right)$ may also be used to express ductility of the metal specimen or sample. Low-carbon steel is one of just a few materials that exhibit a point where the strain increases without an accompanying increase in stress, which poses a problem in deciding when plastic deformation begins for such materials. By agreement, a practical approximation of the elastic limit, called the offset yield strength, is used. It is the stress at which a material exhibits a specified plastic strain. For most applications, a plastic strain of $0.002 \mathrm{in}$./in. can be tolerated, and the stress that produces this strain is the yield strength, sometimes expressed as $0.2 \%$ strain. The yield strength is determined by drawing a straight line, called the offset line, from the $0.2 \%$ strain value on the horizontal axis parallel to the straight-line portion of the stress-strain curve. The stress at which this offset line intersects the stress-strain curve is designated as the yield strength of the material at $0.2 \%$ offset. In some cases the offset can be specified as $0.1 \%$ or even $0.5 \%$. Figure 2.15 shows the results for the ultimate tensile and yield strength obtained from numerous tension specimens for the aluminum 7075-T651 [41]. The tensile properties are normally determined on longitudinal specimens. Generally, they have properties higher than those of transverse specimens. 
A. 7075-T651 MATERIAL

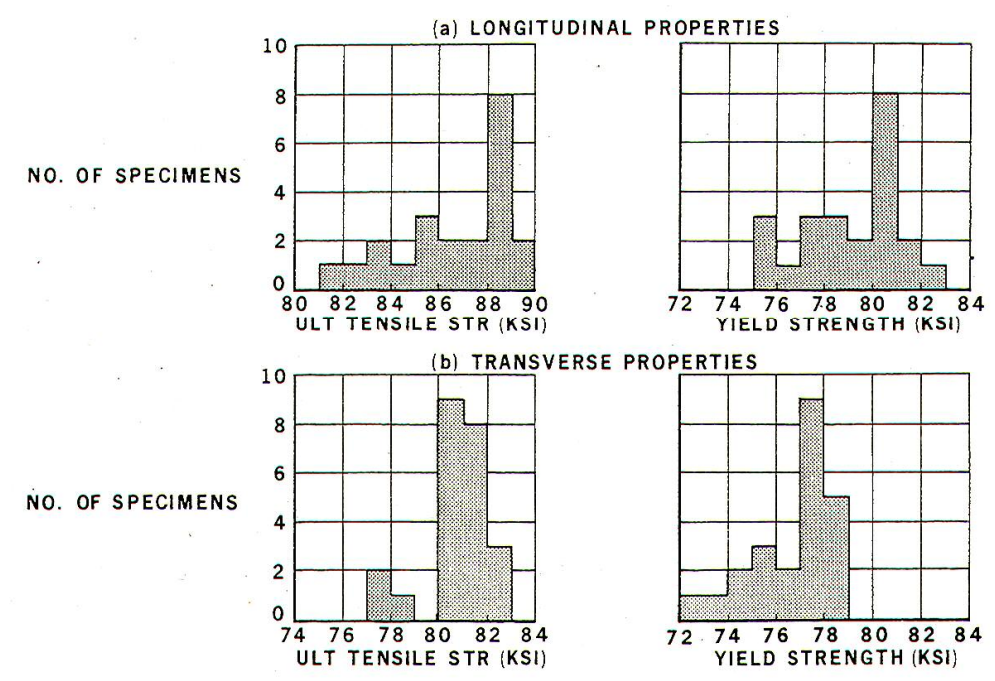

Figure 2.15 Variation of material properties [41].

\subsubsection{Ultimate strength $\left(S_{\text {ult }}\right)$}

Ultimate strength or tensile strength is the maximum stress developed in a material during a tensile test. It is a good indicator of the presence of defects in the crystal structure of a metal material, but it is not used too much in design because considerable plastic deformation occurs in reaching this stress. For brittle materials, tensile strength is still a valid criterion. Most gray cast irons are specified by their tensile strengths. Plastic deformation is not all bad; however, in many applications the amount of plastic deformation must be limited to much smaller values than that accompanying the maximum stress.

At this point it is important to notice that material properties are not exact quantities. There is scatter or variability in the data that are collected from specimen of the same material, in spite of the use of the most precise measuring apparatus, and 
a very efficient controlled test. In fact, a number of identical tensile prepared from the same piece of material, and tested by the same equipment will lead to different stress-strain plots, and to different values of modulus of elasticity, yield strength, and tensile strength values. Among the factors that contribute to this variability are: the test method, variations in specimen fabrication procedures, operator bias and equipment calibration [34].

It is important for the fatigue prediction to realize that variability of material is present and must be taken into account to obtain more realistic results. In this research, the material properties are considered with uncertainty according to some average and coefficient of variation reported in the literature $[38,42]$. They are typically assumed normally distributed for it is a reasonable model for many natural processes or physical properties [43,44]. Table 2.3 shows the values for the aluminum 7075-T651.

Table 2.3 Properties for the aluminum 7075-T651.

\begin{tabular}{|c|c|c|c|}
\hline Parameter & Mean & $\begin{array}{c}\text { Coefficient of } \\
\text { variation }[36]\end{array}$ & Units. \\
\hline$E$ & $1.00 \mathrm{E}+05$ & $5 \%$ & $\mathrm{ksi}$ \\
\hline$S_{\mathrm{y}}$ & 68 & $7 \%$ & $\mathrm{ksi}$ \\
\hline$S_{\mathrm{ul}}$ & 84 & $5 \%$ & $\mathrm{ksi}$ \\
\hline
\end{tabular}

When any material is at rest, it is known that the atomic structure is in equilibrium. The bonding forces in this structure resist any attempt to disrupt this equilibrium. One such attempt may be an external force or load. Stress results from forces such as tension, compression, or shear that pull, push, twist, cut, or in some way deform or change the shape of a piece of material. In the case of aircraft 
structures, they are subjected to load conditions that are characterized by variable amplitude loading. Such structures are subjected to working loads due to takeoffs, maneuvers, and landing, as well as to vibratory loads due to runway roughness and air turbulence, and wind gust loads in storms. In Chapter 3, we will cover the treatment of the load for the fatigue crack growth prediction. 


\section{Chapter 3 Data Collection}

\subsection{Load Spectrum - Background}

Information of the load-time history of an aircraft in service is collected in load spectra, which is the engineering definition of the fatigue environment that a structure experiences throughout its design life. It is defined by the load (or stress) amplitude versus the number of cycles [45]. The need for further research in load spectra started in the aeronautical field as the result of some early accidents. In fact, the accident of a Lufthansa aircraft in 1927 triggered a significant fatigue research by Gassner [46] and Teichmann [47]. As a result of this research, it was understood that aircraft wings were dynamically loaded during flying in turbulent air which resulted in numerous load cycles with quite variable amplitudes, and the need for measuring these loads was recognized.

In the early 1930s, a strain measurement was developed for measuring the load, which consisted in scratch the load-time history with a diamond on glass and analyze it under the microscope [48] and other techniques available by that time. Teichmann analyzed the load-time history statistically in two different ways. First, he considered maxima and minima as the relevant data of a load-time history, then the statistical data was restricted to counting these peak values in specified intervals, which lead to a one-dimensional spectrum. Later, Teichmann defined a statistical counting of ranges between successive maxima and minima and considered different values of these ranges, which then lead to a two-dimensional spectrum. 
In later years, other load measurement techniques were developed as well as statistical evaluation methods for the colleted data. In the 1950s, a new technique was introduced to measure accelerations in the centre of gravity of the aircraft from which the loads on the structure were calculated. Also, The Royal Aircraft Establishment in the UK [49] developed a counting accelerometer, also known as "fatigue meter", which counted the number of times an acceleration level was exceeded. Figure 3.1 shows an example of this technique. However, the precision of the calculation of loads in the center of gravity of the aircraft was not always realistic or sufficiently accurate. Strain gauges were then used to measure the load history on a fatigue critical component, which are designed to convert mechanical motion into an electronic signal. A change in capacitance, inductance, or resistance is proportional to the strain experienced by a sensor. If a wire is held under tension, it gets slightly longer and its cross-sectional area is reduced. This changes its resistance $(\mathrm{Re})$ in proportion to the strain sensitivity (Se) of the wire's resistance. When a strain is introduced, the strain sensitivity, which is also called the gage factor (GF), is given by [50],

$$
G F=\left(\frac{\Delta \operatorname{Re}}{\operatorname{Re}}\right) /\left(\frac{\Delta L}{L}\right)=\left(\frac{\Delta \operatorname{Re}}{\operatorname{Re}}\right) / \text { Strain }
$$

Techniques based on strain gage showed long-term reliability under various service conditions. 


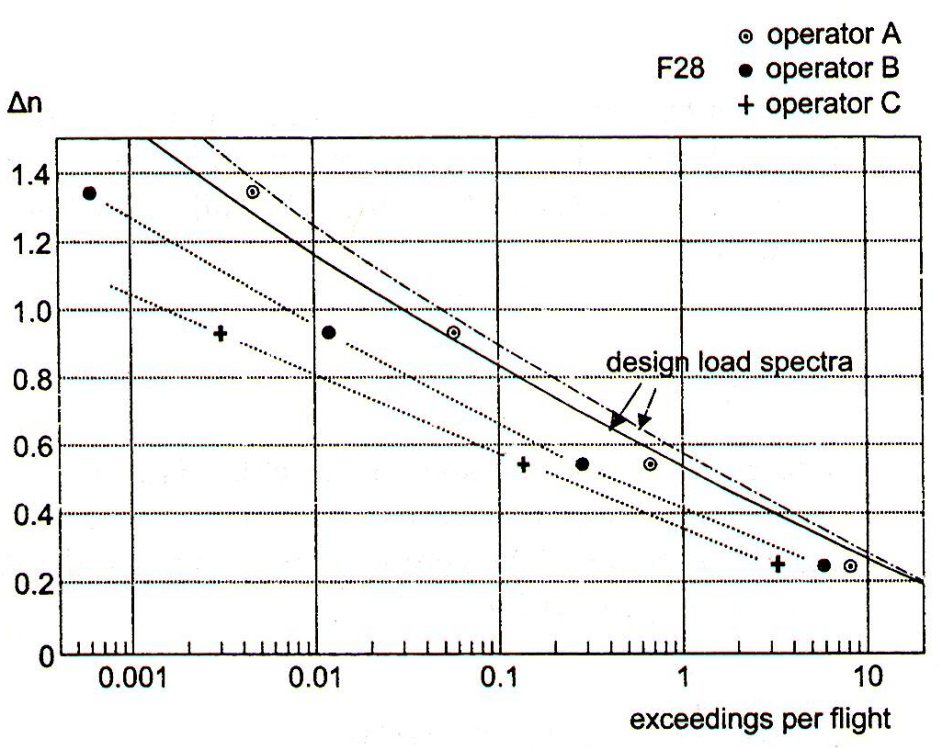

Figure 3.1 Gust load spectra measured with counting accelerometers compared to the design load spectrum of the Fokker F-28 [33].

\subsection{Calculation of Spectrum Load}

In developing a fatigue spectrum, one must define all loading events that a structure will experience and the number of times that each event will occur. In the case of an aircraft, each flight profile may be divided into different events, such as taxi, takeoff, ascent, cruise, descent, landing, and taxi after landing. Figure 3.2 shows this profile. 


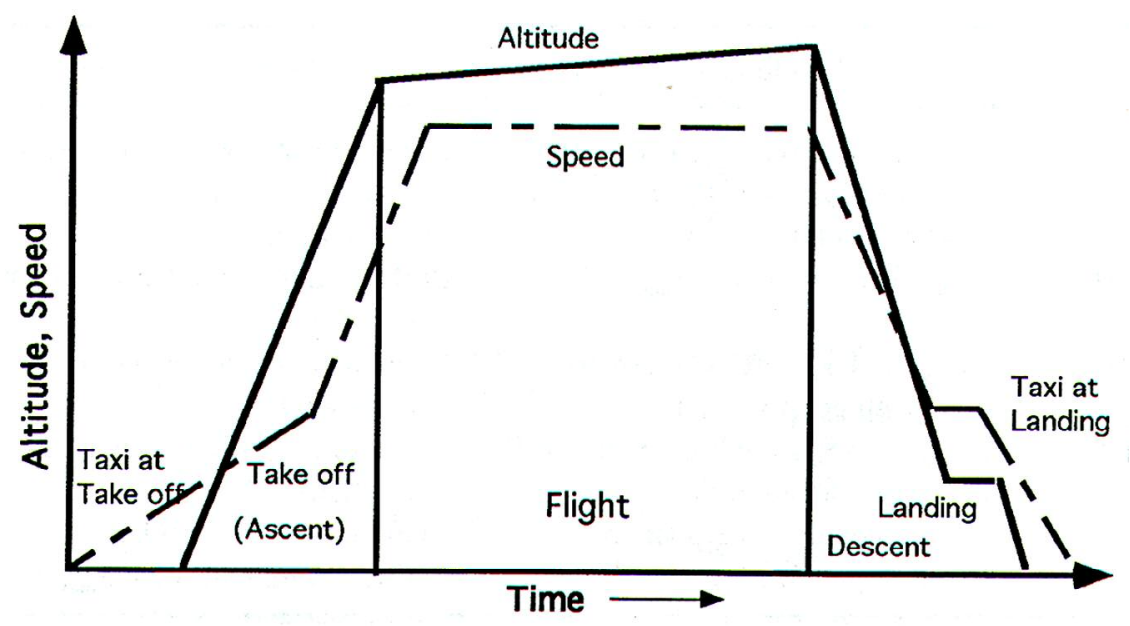

Figure 3.2 Mission profile [45].

The fatigue spectrum for each event is a function of several variables. For instance, the wing load magnitude and its cyclic behavior during ascent may depend on speed, weight, and gust factor. Figure 3.3 shows a simplified version of a complex load spectrum for the wings of a transport aircraft during each flight.

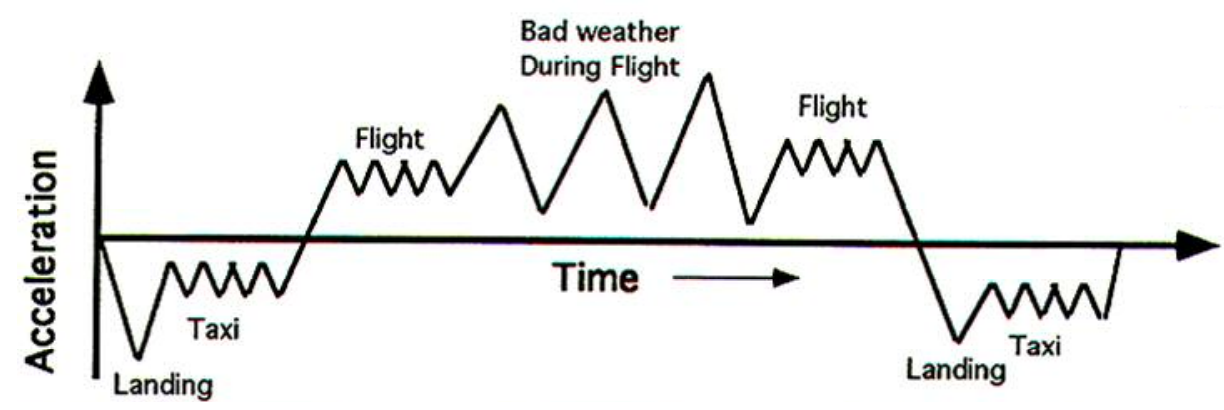

Figure 3.3 Simplified load spectrum for an aircraft [45].

Load spectrum is determined differently depending on whether the loading event is static or dynamic. In the case of the former, it can be established with relative ease. A spur gear that is designed to actuate the wing flaps of an aircraft 
during ascent would undergo a fixed number of complete revolutions under a constant torque. The stress time history for a typical gear tooth during each ascent event can be calculated using the equations of static equilibrium. However, in situations where flexible structures and fast changing loads are involved, the structure or components react dynamically to the loading environment. The cyclic behavior of the time history would be a function of both the load variation and the dynamics of the structure.

The time history for each event is established using a cycle counting procedure. In the literature, it is possible there are different procedures such as rain flow counting, peak counting, level crossing and range-pair counting. The most recognized and most widely used is the rain flow method. In all of the above mentioned cases, the irregular load sequence can be converted to a sum of cycles with different stress amplitudes that assess the total damage induced in a given part.

Once the time history for each event is established, it must then be converted to a fatigue spectrum consisting of load range and mean range versus number of cycles, where range is defined as the algebraic difference between successive valley and peak loads.

For the component analyzed in this research, the load in the form of stress spectrum was collected at the dome nut hole locations at Fillet Fairing for each flight. Figure 3.4 indicates the general locations. 


\section{Lower Surface Dome Nuts at Fillet Fairing}
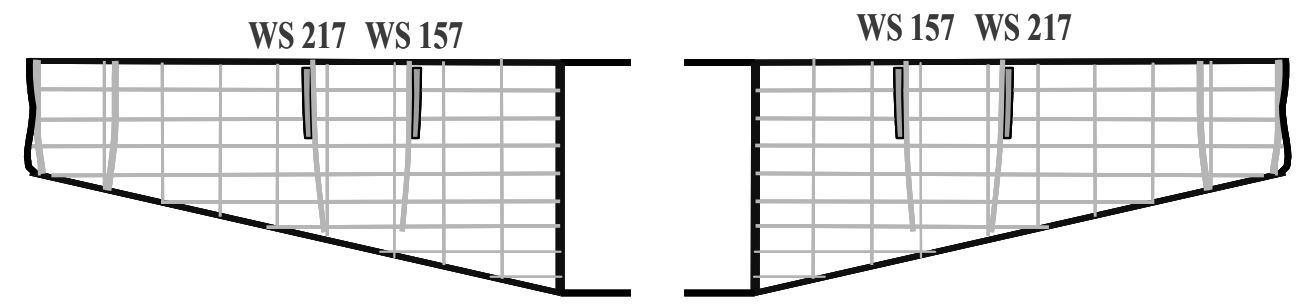

Figure 3.4 Dome nut hole locations.

The data was collected using the SAFE (Structural Appraisal of Fatigue Effects) life methodology [17] within which individual aircraft tracking is a requirement. The SAFE methodology was explained in Chapter 1. However, it is mentioned again, in this research the stress history as a function of the Fatigue Life Expended Index (FLEI) is used as the time-damage agent for aging. A partial example sample of this data appears in Table 3.1. A description of the flight in line one, followed by data in subsequent lines indicating the peaks and valleys of each stress. Each flight comprises about one million peaks and valleys.

Table 3.1 Flight and stresses description.

\begin{tabular}{|c|c|c|c|c|c|c|c|c|}
\hline Flt No & Flt Date & $\begin{array}{c}\text { Flight } \\
\text { Duration } \\
\text { [hours] }\end{array}$ & \multicolumn{2}{|c|}{$\begin{array}{l}\text { \# Full Stop } \\
\text { Landings }\end{array}$} & \multicolumn{2}{|c|}{$\begin{array}{c}\text { \# Touch \& } \\
\text { Go Landings }\end{array}$} & \multicolumn{2}{|c|}{$\begin{array}{l}\# \text { of stress points in } \\
\text { the flight spectrum }\end{array}$} \\
\hline 1 & 01-Feb-73 & 4.4 & \multicolumn{2}{|l|}{1} & & 2 & \multicolumn{2}{|c|}{1148} \\
\hline $\begin{array}{l}\text { Max } \\
{[p s i]}\end{array}$ & $\begin{array}{l}\text { Min } \\
{[\mathrm{psi}]}\end{array}$ & $\begin{array}{l}\text { Max } \\
\text { [psi] }\end{array}$ & $\begin{array}{l}\text { Min } \\
{[p s i]}\end{array}$ & & & $\begin{array}{l}\text { Min } \\
{[\mathrm{psi}]}\end{array}$ & $\begin{array}{l}\text { Max } \\
\text { [psi] }\end{array}$ & $\begin{array}{l}\text { Min } \\
\text { [psi] }\end{array}$ \\
\hline 10290.2 & 6561.4 & 10766.2 & 6561.4 & 113 & 21.5 & 6561.4 & 10434.3 & 7855.2 \\
\hline 9382.8 & 5334.6 & 9382.8 & 5792.9 & 106 & 34.4 & 8021.7 & 9571.4 & 6471.9 \\
\hline 9901.7 & 6640.7 & 9502.2 & 6397.4 & &.. & $\ldots$ & $\ldots$ & $\ldots$ \\
\hline
\end{tabular}


The load spectrum is calculated using a MatLab ${ }^{\circledR}$ routine developed in this research. The routine is based on the standard ASME E-1049 "Standard Practices for Cycle Counting in Fatigue Analysis" [51]. The results of this calculation are used as variables in both the crack initiation phase, and the crack growth phase. In this study only crack growth is modeled, as it analyzes all aircrafts passed the $100 \%$ FLE, an index that has been assumed as a point where some crack growth phenomenon initiates.

Table 3.2 shows a summary of spectrum statistics related to each of the 25 aircrafts analyzed in this research. The data are not accurate loads of specific aircrafts. The load spectrum contains between 281,479 and 1,109,450 cycles.

Table 3.2 Spectrum summary for the 25 aircrafts.

\begin{tabular}{|c|c|c|c|c|c|}
\hline Aircraft & $\begin{array}{c}\text { Max stress } \\
{[\mathrm{ksi}]}\end{array}$ & $\begin{array}{c}\text { Min Stress } \\
{[\mathrm{ksi}]}\end{array}$ & $\begin{array}{c}\text { Median } \\
{[\mathrm{ksi}]}\end{array}$ & $\begin{array}{c}\text { Mean } \\
{[\mathrm{ksi}]}\end{array}$ & $\begin{array}{c}\text { SD } \\
{[\mathrm{ksi}]}\end{array}$ \\
\hline 1 & 24.2 & -9.4 & 10.8 & 8.6 & 4.2 \\
\hline 2 & 25.0 & -8.0 & 11.1 & 9.2 & 4.0 \\
\hline 3 & 25.4 & -9.4 & 11.0 & 9.5 & 4.1 \\
\hline 4 & 24.2 & -6.7 & 10.5 & 9.8 & 3.9 \\
\hline 5 & 25.2 & -8.8 & 11.2 & 9.1 & 4.5 \\
\hline 6 & 24.1 & -8.0 & 11.5 & 9.1 & 4.3 \\
\hline 7 & 23.5 & -6.5 & 11.4 & 9.4 & 4.0 \\
\hline 8 & 25.5 & -7.8 & 11.3 & 9.4 & 4.1 \\
\hline 9 & 23.8 & -7.3 & 11.8 & 9.3 & 3.8 \\
\hline 10 & 26.0 & -7.1 & 11.3 & 9.3 & 4.8 \\
\hline 11 & 22.7 & -7.5 & 11.8 & 9.2 & 4.2 \\
\hline 12 & 24.7 & -8.8 & 11.5 & 9.5 & 3.8 \\
\hline 13 & 26.8 & -9.3 & 11.4 & 9.1 & 3.9 \\
\hline 14 & 23.9 & -9.1 & 12.0 & 9.4 & 4.5 \\
\hline 15 & 23.4 & -6.5 & 10.8 & 9.3 & 4.1 \\
\hline 16 & 22.7 & -7.0 & 11.6 & 9.7 & 3.9 \\
\hline 17 & 26.1 & -6.5 & 10.7 & 10.2 & 3.8 \\
\hline 18 & 24.1 & -8.4 & 11.8 & 9.2 & 3.7 \\
\hline 19 & 23.8 & -7.3 & 11.3 & 8.5 & 4.2 \\
\hline 20 & 26.0 & -6.8 & 13.1 & 9.6 & 3.7 \\
\hline
\end{tabular}




\begin{tabular}{|l|l|l|l|l|l|}
\hline 21 & 24.1 & -6.7 & 11.5 & 9.4 & 5.5 \\
\hline 22 & 23.0 & -6.4 & 11.4 & 8.8 & 4.7 \\
\hline 23 & 23.9 & -7.4 & 11.9 & 9.0 & 3.7 \\
\hline 24 & 22.5 & -9.0 & 11.3 & 8.5 & 5.1 \\
\hline 25 & 24.4 & -6.4 & 12.2 & 9.0 & 5.0 \\
\hline
\end{tabular}

From Table 3.2, it is important to notice that for all aircrafts the mean stresses is below the 0.8 Sy design requirement of LEFM.

\subsubsection{Fatigue Life Expended Index $(F L E)$}

To allow a fleet manager to optimize the maintenance of the aircrafts, a fatigue index is used, rather than the traditional, and less accurate, flight hour basis.

The aircrafts are fatigue monitored using strain data recorded by sensors installed at different critical locations on the structure. The strain data is processed using a fatigue life prediction program to calculate the damage that has accumulated by each aircraft. The result is a Fatigue Life Expended index (FLE) which compares the amount of damage accumulated by each aircraft to the damage experienced by a representative fatigue test article. The SAFE process which calculates a Fatigue Life Expended index (FLE) for individual aircraft in the fleet is defined below [52]:

$$
F L E=\sum \text { Calculated Aircraft Fatigue Damage } \cdot \mathrm{SF}_{1}
$$

where

$\mathrm{SF}_{1}$ is the scatter factor applied by the fleet operator.

The FLE is the complement of fatigue-life remaining. As such, an FLE of 100 percent is equal to not fatigue-life remaining. The FLE index limit of $100 \%$ is determined by a full-scale fatigue test (FSFT). In the FSFT the time to reach a crack 
size of 0.01 inches is determined. This time is specified as test demonstrated life. Then the FLE value of $100 \%$ corresponds to half the time of the test-demonstrated life. In other words, an FLE value of $100 \%$ corresponds to a damage accumulation of one half of the value predicted by the Miner's Rule [53].

For the prediction of the fatigue crack growth life, an initial crack size distribution assumption must be made. The following section will explain how this initial crack size is obtained.

\subsection{Initial Crack Size Distribution}

Structural components inevitably suffer from flaw or crack defects, such as surface scratches, surface roughness or weld defects of random sizes, which usually occur during the manufacturing and handling process [54]. These defects are shown to have a detrimental effect on the fatigue life of the structural components by promoting crack initiation sites. In order to make reliable predictions, data regarding the initial flaws size must be known. However, the Non Destructive Inspection methods (NDI) available cannot provide the adequate information concerning the statistical distributions of initial flaws. Consequently, two concepts have been developed and have been proven useful as design tools for making predictions for aircraft structural reliability problems. These methods are: the equivalent initial flaw size distribution and the distribution of time-to-crack initiation. 


\subsubsection{Equivalent Initial Flaw Size}

Initial flaws of a high quality structure are not detectable. For this reason, the equivalent initial flaw size concept was introduced by Gray and Rudd [55] and developed by Yang and Manning [56]. Once the initial flaw size distribution is established, the fatigue crack growth can be estimated without further experimental tests.

The initial flaw size is an artificial crack size, which is derived from the distribution of fatigue crack occurring later on during service life. The distribution of initial flaw size is determined by back-extrapolating this distribution of fatigue cracks according to a master crack growth function to zero time (zero damage) or some reference time serving to represent the initial time of the assessment. Therefore, the initial flaw size will result in an actual fatigue crack at a point in time when it is grown forward.

The distribution of fatigue cracks at a particular time can be difficult and costly to determine. This kind of information usually requires a tear down inspection, possibly following a full-scale fatigue test or from retired airframes. Fatigue cracks detected during in-service inspections of structural components or fatigue cracks obtained in laboratory coupon testing may also serve as a starting point for developing the initial flaw size distribution.

\subsubsection{Time-To-Crack Initiation}

The period of crack initiation or the time-to-crack initiation is defined as the time in cycles flights, or flight hours it takes for a non detectable crack from the 
beginning of fatigue loading to grow to a reference crack size. This crack initiation distribution is physically observable and can be obtained by experiments and test results.

In some instances, the time-to-crack initiation period makes up a large proportion of the crack growth life of a structural component and this is especially the case for jet engine disc components [57]. The reference crack size is commonly selected on the basis of a detectable crack by NDI technique.

In order to determine the initial flaw size distribution, the test results of the time-to-crack initiation are produced and through a transformation, the initial flaw size distribution is derived, (see section 3.2.1.) The relation between the time-tocrack initiation distribution and initial flaw size distribution can be visualized in Figure 3.5. Yang et al. [56] have demonstrated existence of compatibility between the time-to-crack initiation and initial flaw size distributions function for the Weibull and the lognormal distributions. Since accurate crack growth is almost impossible to predict at the small crack size, a power law matching the crack growth rate is used to reflect the crack growth law transforming the time-to-crack initiation distribution function back to the y-axis at zero time to produce a compatible initial crack size distribution. 


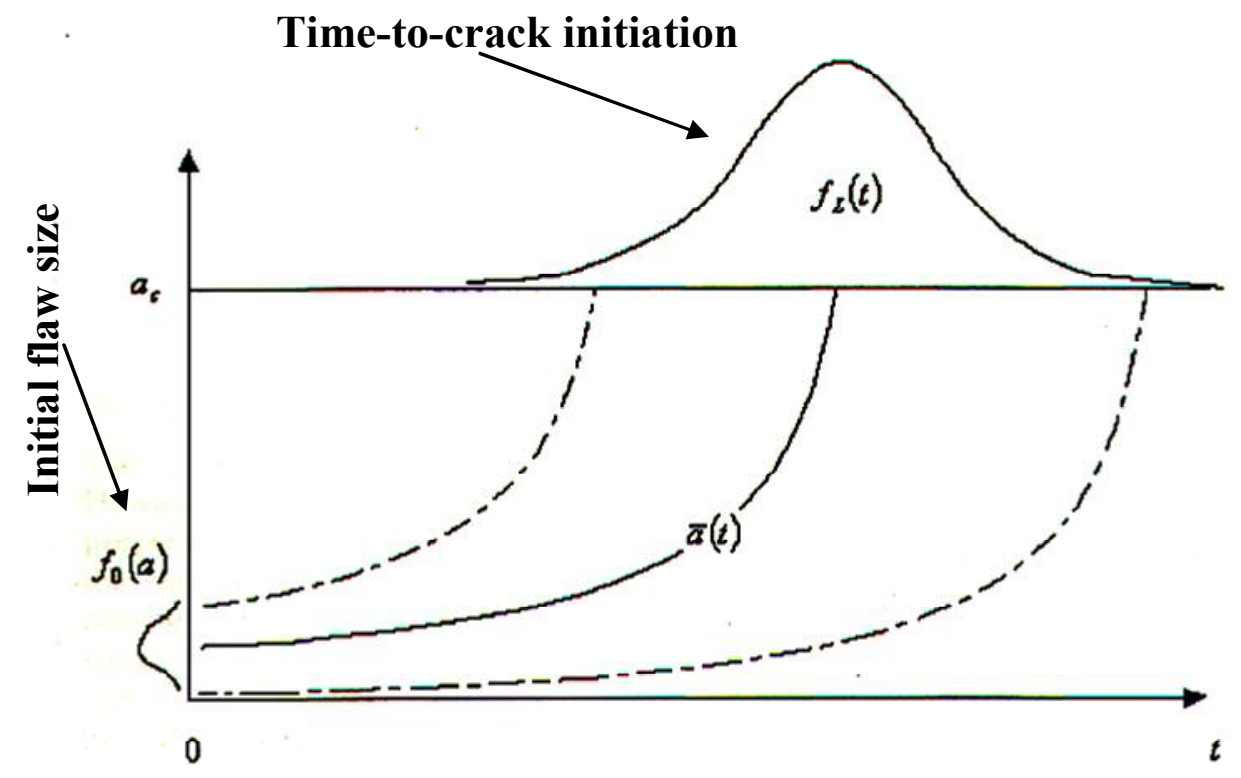

Figure 3.5 Process showing the compatibility between time-to-crack initiation and initial flaw size $p d f[42]$.

The Weibull distribution is one of the most commonly used distributions for representing the crack size distribution. Many applications indicated that this distribution fits particular well to the tail of the large crack size data found in teardown inspections and maintenance inspections. However, the selection of the probability distribution should be based on how well it fits the data.

In this research, we are interested in a methodology to estimate the initial flaw size distribution function when a $100 \%$ FLE has been reached. The fundamental military characterization of the initial cracks at $100 \%$ FLE, assume that the probability of a crack size of 0.01 inch or larger is less than 1/1000. A lognormal distribution seems to be good fit to represent the prior distribution in a Bayesian 
updating. This initial crack size is characterized by the parameters, $\log$ mean $\mu_{\mathrm{t}}=-$ 4.71 and $\log$ S.D. $\sigma_{\mathrm{t}}=3.59 \mathrm{E}-02$. Figure 3.6 shows a plot of such a $p d f$.

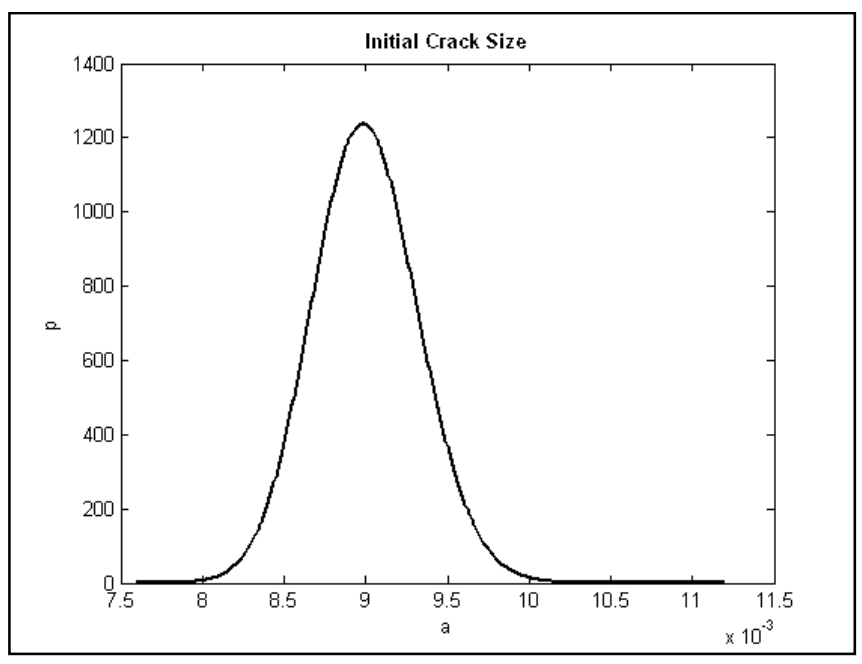

Figure 3.6 Estimated Initial Crack Size $p d f$.

\subsection{Crack Observation (Evidence)}

Conventional inspection is based on either visual inspection or one of the different NDI methods developed in the mid-50s and initially used in the early to the mid-60s. Frequent visual inspections can be rapidly and easily performed on a variety of structures. Visual inspection is particularly valuable in nondirected or general inspections or in those inspections in which no previous damage is suspected. When fatigue test or in-service experience indicate that a directed structural inspection is required, instrumented NDI methods become valuable since they can detect smaller cracks and require only minimal disassembly [58]. Figure 3.7 shows a distribution of cracks found in service depending on the inspection method. In this research, a directed structural inspection is the approach used to obtain the evidence. 

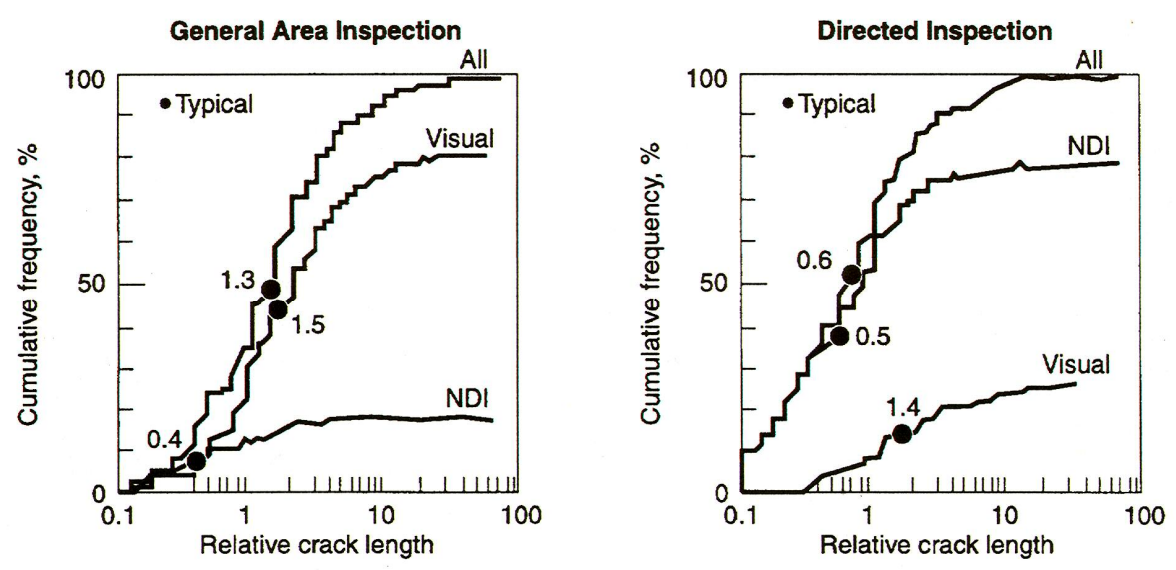

Figure 3.7 Distribution of cracks found in service [58].

NDI is one of the elements of the Aircraft Structural Integrity Program (ASIP)[3], which also includes tools such as teardown inspection, full scale fatigue testing, component testing, and testing of structural parts. There are five major NDI techniques: magnetic particle (MT), liquid penetrant (PT), ultrasonic (UT), eddy current (ET) and radiography (RT). New techniques such as thermography and shearography have recently emerged.

All these NDI techniques are used in the location being analyzed in this research, the Dome Nut and Rivet Hole Geometry at FC351 area of each aircraft. Figure 3.8 shows the location of the cracks. 


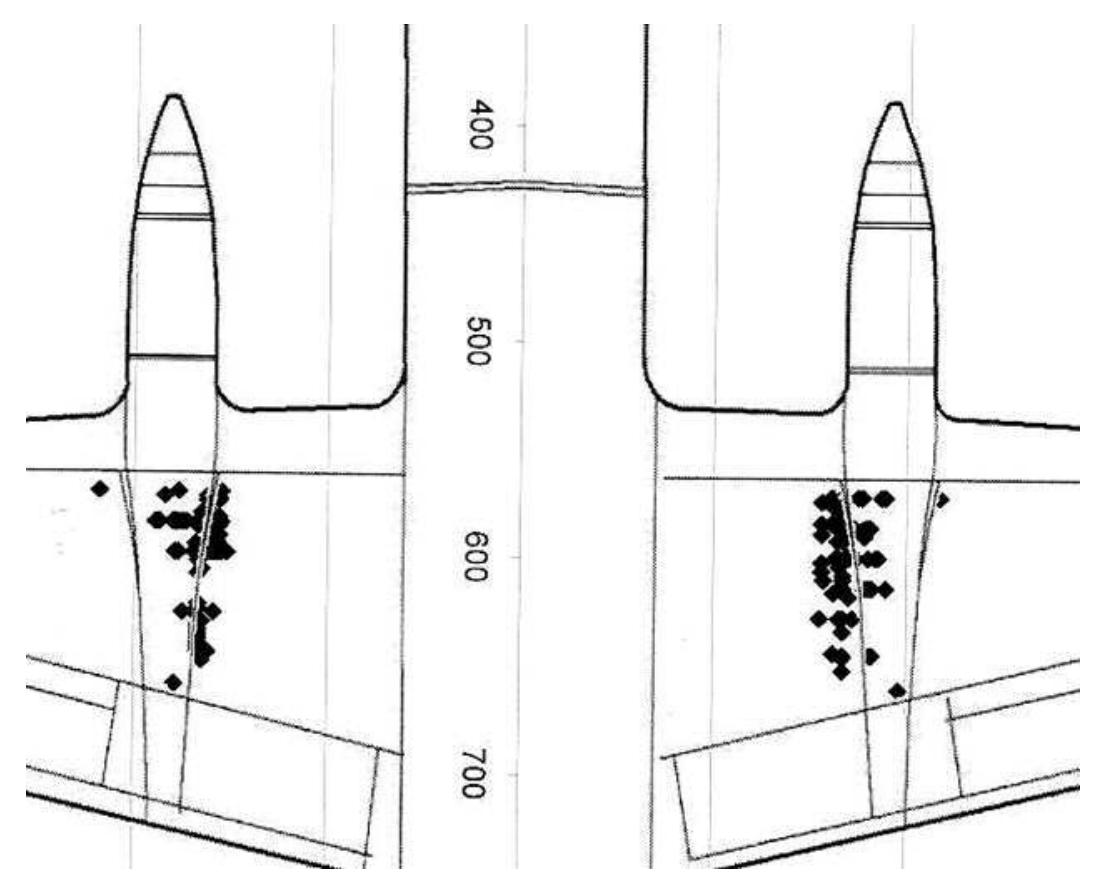

Figure 3.8 Cracks location at the FC 351 area.

Ideally the exact crack sizes would be the best form for reporting observed crack size evidence. However, the findings have been reported according to the format shown in Figure 3.9. As such, the cracks are assigned one of four sizes of extra small, small, large and extra large and the database reports how many of such cracks in each bin has been observed for each aircraft inspected.

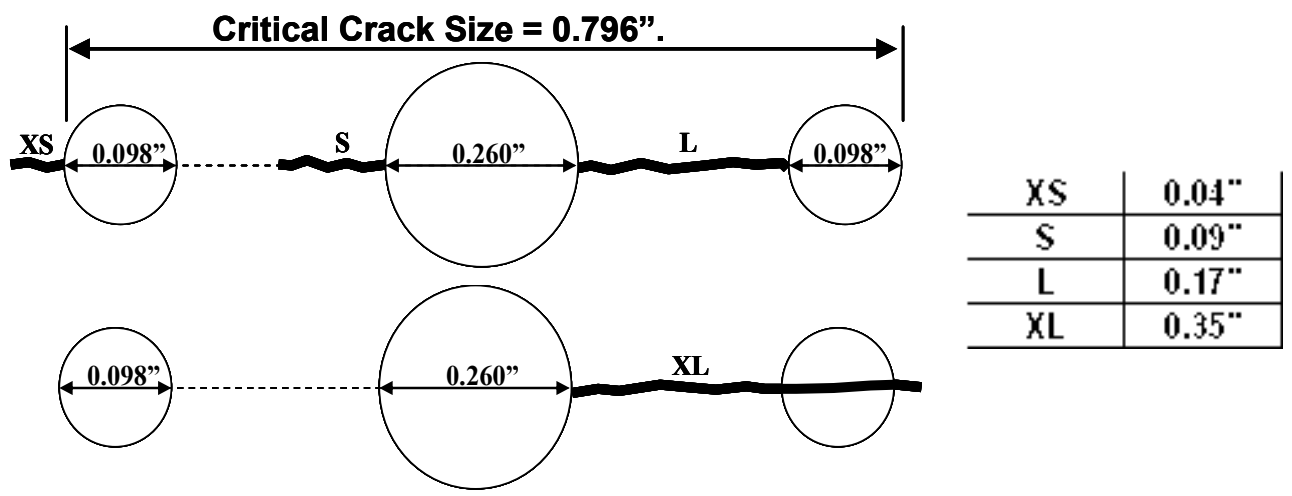

Figure 3.9 Inspection cracks size. 
Table 3.3 shows the finding results (evidence) to be used as evidence to build the likelihood probability function in the Bayesian updating step. This will be discussed in chapter 6 .

Table 3.3 Observed number of various crack sizes for the 25 aircrafts.

\begin{tabular}{|c|c|c|c|c|}
\hline & \multicolumn{4}{|c|}{ Crack Size } \\
\hline Aircraft & XS & S & L & XL \\
\hline 1 & 1 & 1 & 0 & 0 \\
\hline 2 & 8 & 0 & 0 & 0 \\
\hline 3 & 2 & 0 & 0 & 0 \\
\hline 4 & 0 & 7 & 0 & 0 \\
\hline 5 & 3 & 0 & 0 & 0 \\
\hline 6 & 0 & 1 & 0 & 0 \\
\hline 7 & 4 & 0 & 0 & 0 \\
\hline 8 & 0 & 3 & 0 & 3 \\
\hline 9 & 1 & 0 & 0 & 0 \\
\hline 10 & 0 & 3 & 0 & 1 \\
\hline 11 & 0 & 0 & 0 & 1 \\
\hline 12 & 3 & 2 & 0 & 0 \\
\hline 13 & 3 & 1 & 0 & 2 \\
\hline 14 & 42 & 0 & 0 & 0 \\
\hline 15 & 6 & 0 & 0 & 0 \\
\hline 16 & 6 & 0 & 0 & 0 \\
\hline 17 & 4 & 1 & 0 & 0 \\
\hline 18 & 3 & 0 & 0 & 0 \\
\hline 19 & 7 & 0 & 0 & 0 \\
\hline 20 & 4 & 5 & 0 & 0 \\
\hline 21 & 0 & 2 & 0 & 0 \\
\hline 22 & 0 & 1 & 0 & 0 \\
\hline 23 & 5 & 2 & 2 & 0 \\
\hline 24 & 8 & 0 & 0 & 0 \\
\hline 25 & 2 & 2 & 0 & 4 \\
\hline
\end{tabular}




\section{Chapter 4 An Integrated Approach for Assessing the \\ Crack Growth Under Variable Amplitude \\ Loading}

\subsection{Crack Growth Assessment}

Assessing crack growth and the lifetime of the aircrafts under variable amplitude loading can be done using either the global (characteristic) approach or cycle-by-cycle approach. The global approach is based on the statistical description of the load spectrum [59], while the cycle-by-cycle approach is based on the sum of cyclic damage or crack advance associated with each cycle. This research focuses on the cycle-by-cycle approach describes the crack growth models, which consider the stress ratio effect and the interaction effect. It is important to note that the cycle-bycycle approach can be used by either taking into account interaction effects $[1,29,59]$ or by ignoring such effects. Figure 4.1 illustrates the two approaches.

The global approach, which has been used in a number of fatigue-critical applications such as the fatigue of steel bridges, will be explained briefly. In some applications such as flight simulation of gust loading spectra for aircraft, random loading and its statistically equivalent program loading are known to provide different results [1]. 


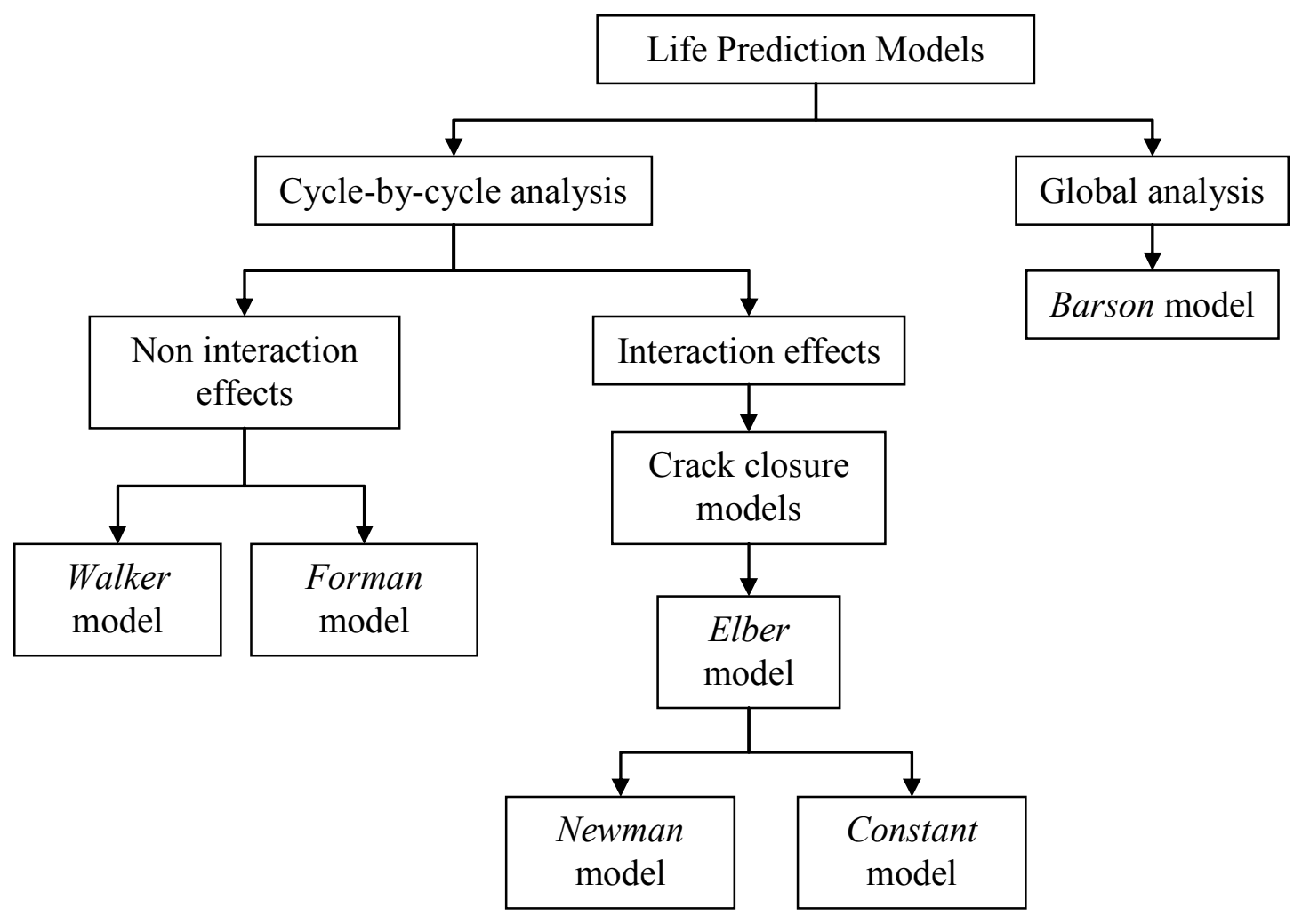

Figure 4.1 Life prediction models.

\subsubsection{Global Approach}

This model was first proposed by Paris [1] for random loading, and it is based on the hypothesis that random variation of the crack tip fields are describable in terms of the root-mean-square value of the stress intensity factor range, $\Delta K_{\text {rms. }}$ The variable amplitude crack growth rates are given by the Paris relationship:

$$
\frac{d a}{d N}=C\left(\Delta K_{\mathrm{rms}}\right)^{m}
$$

where $C$ and $m$ are material constants and

$$
\Delta K_{\mathrm{rms}}=\frac{\sqrt{\sum_{i=1}^{n} \Delta K_{i}^{2}}}{n}
$$


where $\Delta K_{i}$ is the stress intensity factor range in the ith cycle in a sequence consisting of $n$ stress cycles. For constant amplitude, $\Delta K_{\mathrm{rms}}=\Delta K$.

\subsubsection{Cycle-by-Cycle Approach}

The Palmgren-Miner model is the most well-known cycle-by-cycle approach, in which load interaction effects are not considered. The cycle-by-cycle summation theory, extended to damage crack growth, assumes that the crack growth increment per cycle is equal to the crack growth rate associated with constant amplitude loading of the same magnitude; however, it may also be deduced from a load interaction model [1]. In such model, the crack growth $\Delta a$ in each individual cycle can be calculated in two different ways:

1. by estimation from the $\mathrm{d} a / \mathrm{d} N$ Vs. $\Delta K$ curve of the material, or

2. by integration where $a$ is the dependent variable.

If the current crack length is $a_{j}$ and the increment is $\Delta a_{j}$, the new value of crack length for the next cycle is

$$
a_{j+1}=a_{j}+\Delta a_{j}=a_{j}+\left(\frac{d a}{d N}\right)_{j}
$$

by denoting the initial crack length as $a_{0}$, we obtain a prediction for the whole spectrum,

$$
a=a_{0}+\sum_{i=1}^{N} f(\Delta K, R, \ldots)=a_{0}+\sum_{i=1}^{N} \Delta a_{i}
$$


Figure 4.2 shows the logic diagram for damage crack growth computation used in this work.

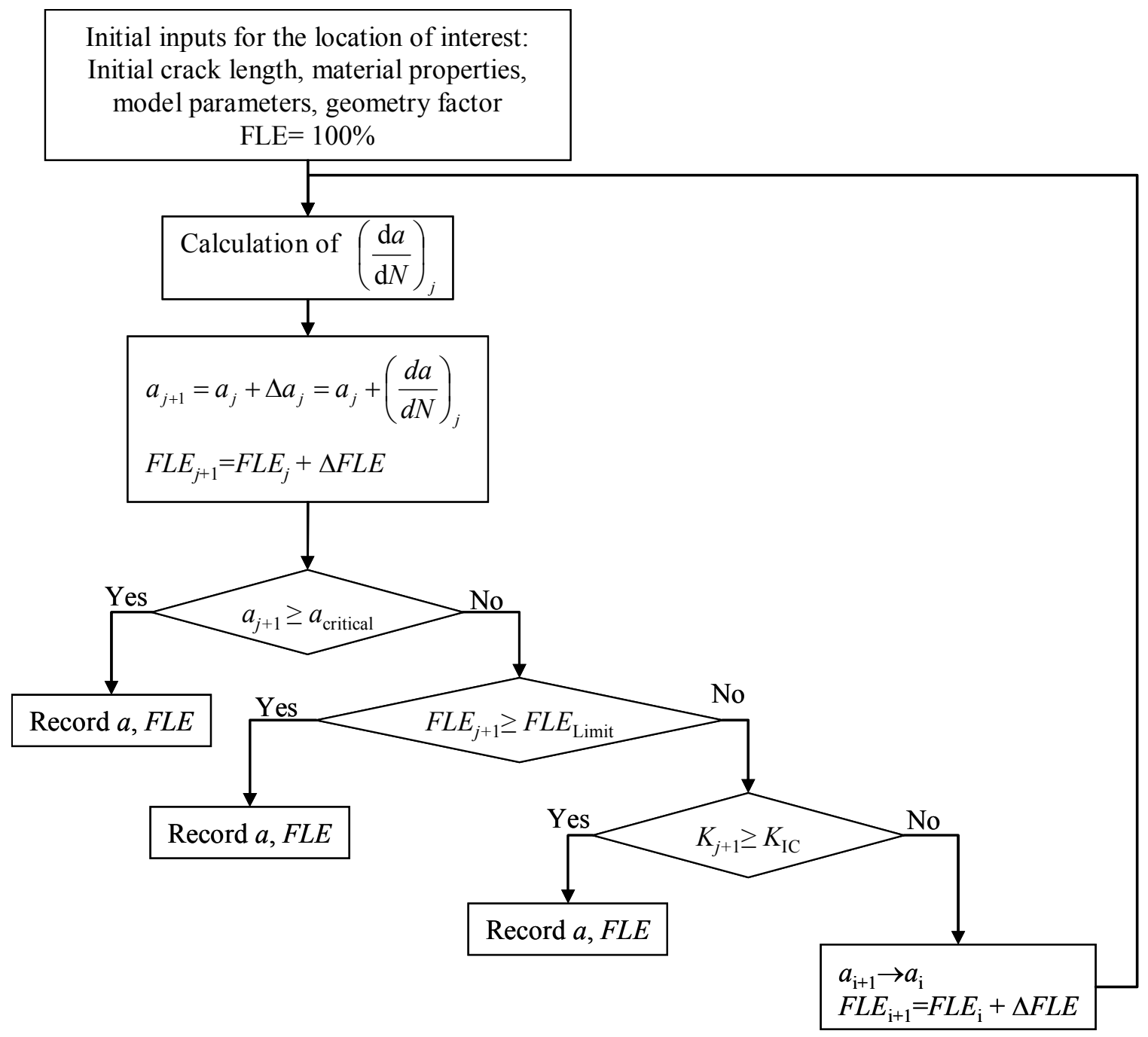

Figure 4.2 Logic diagram for damage crack growth.

Models used in the cycle-by-cycle approach fall into two groups:

- Non-interaction (Walker and Forman models)

- Interaction (crack closure model) 


\subsection{Non-interaction models}

In these models, the main assumption is that growth for a given cycle is not affected by the prior history of loads. That is, the load sequence effect is not considered. These models however, lead to conservative estimates since interaction effects retard crack growth [38,39]. An integration procedure that makes use of a semi-empirical retardation model is more complicated. It means that the prediction should include evaluation of residual stresses and crack closure.

Two of the most popular non-interactions models are the Walker and Forman models, which are described in the following sections.

\subsubsection{Walker Model}

Walker's crack growth equation [60] is the extension of the Paris relationship, but it accounts for the effect of the mean stress ratio, $R$, in region II.

The notion that stress should be related to crack-growth rate and crack length was, first developed by Brock and Schijve [61], McMillan and Pelloux [62], and Erdogan [63],

$$
\frac{\mathrm{d}(2 a)}{\mathrm{d} N}=f\left[\beta\left(S_{\text {max }} \sqrt{2 a}\right)^{c}(\Delta S \sqrt{2 a})^{b}\right]
$$

this equation can be rewritten as,

$$
\frac{\mathrm{d}(a)}{\mathrm{d} N}=f\left\{\left[\beta\left(S_{\text {max }}^{1-\gamma}\right)\left(\Delta S^{\gamma}\right) \sqrt{\pi a}\right]^{\omega}\right\}
$$

where

$1-\gamma=c /(c+b)$ 
$\gamma=b /(c+b)$

$\omega=c+b$

$\omega$ and $\gamma$ are exponents, and $\gamma$ is assumed to have a single value for both crack propagation and fatigue life.

Since product of the two stress terms in Equation 4.6 is a stress, and effective stress can be defined as the following [60],

$$
\bar{S}=S_{\max }^{1-\gamma} \Delta S^{\gamma}=S_{\max }(1-R)^{\gamma}
$$

then, crack growth can be written in the form,

$$
\frac{\mathrm{d}(a)}{\mathrm{d} N}=f[\beta \bar{S} \sqrt{\pi a}]=f(\overline{\Delta K})
$$

where $\overline{\Delta K}$ is an equivalent zero-to-tension $(R=0)$ stress intensity that causes the same growth rate as the actual $K_{\max }, R$ combination. Figure 4.3 shows cracking-rate data for 2024-T3 and 7075-T6 aluminum [61] using $\overline{\Delta K}$ as abscissa for different values of $R$. Values of $\gamma$ used in computing $\overline{\Delta K}(0.5$ for 2024-T3 aluminum and 0.425 for 7075 -T6 aluminum) were determined for best fit using available computer routines. It is important to note, that the effect of stress ratio as accounted for by $\bar{S}$ is independent of the environment [64]. 


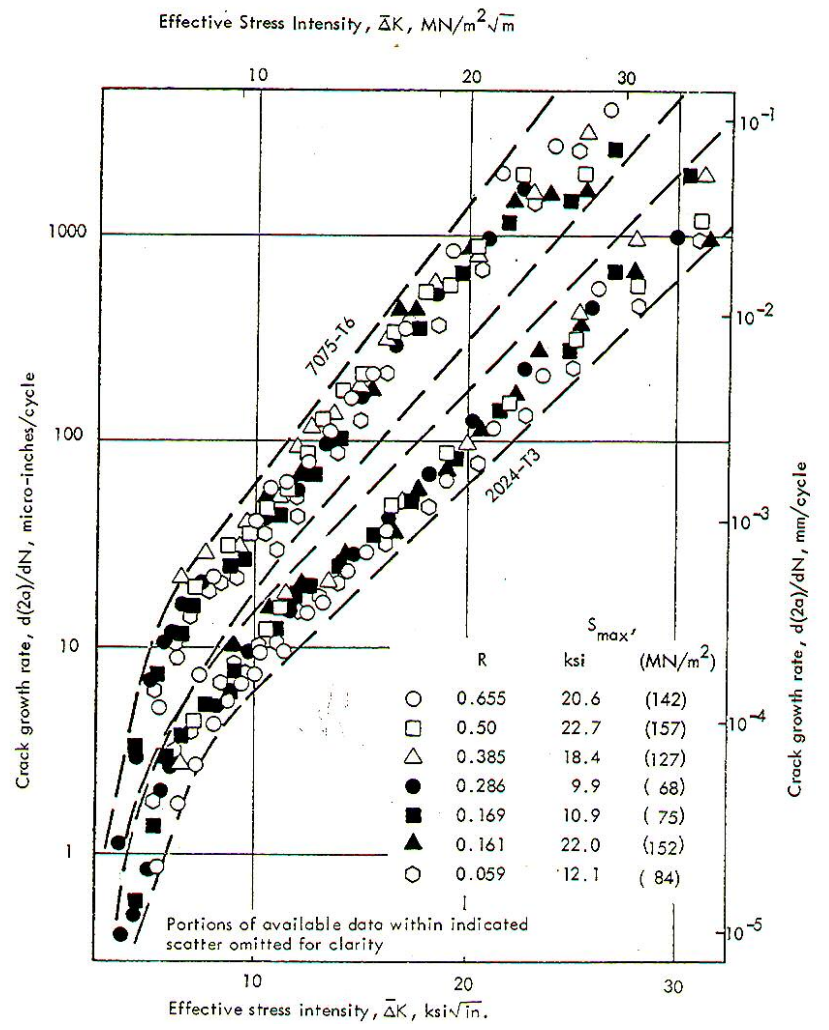

Figure 4.3 Crack growth in 2024-T3 and 7075-T6 [60].

From the above derivation, we can write Equation 4.7 in terms of stress intensity factor,

$$
\overline{\Delta K}=K_{\max }(1-R)^{\gamma}
$$

By substituting Equation 4.7 and Equation 4.9 in the classical crack growth model (Equation 2.7), and denoting $C$ and $m$ as $C_{1}$ and $m_{1}$ for the special case of $R=0$,

$$
\frac{\mathrm{d} a}{\mathrm{~d} N}=C_{1}\left(\beta S_{\max }(1-R)^{\gamma} \sqrt{\pi a}\right)^{m_{1}}
$$

constant $C_{1}$ can be calculated by the following equation,

$$
C_{1}=\frac{C_{1}^{*}}{(1-R)^{m_{1}(1-\gamma)}}
$$


where, $C_{1}^{*}$ and $m_{1}$ are material constants.

Parameters $m_{1}, C_{1}^{*}$ and $\gamma[38]$ are sampled from normal distributions with the characteristics described in Table 4.1.

Table 4.1 Walker parameters.

\begin{tabular}{|c|c|c|c|}
\hline Parameter & Mean & $\begin{array}{c}\text { Coefficient of } \\
\text { variation }\end{array}$ & Units. \\
\hline$m_{1}$ & 3.70 & $5 \%$ & Dimensionless \\
\hline$C_{1}^{*}$ & $1.51 \mathrm{E}-09$ & $7 \%$ & $\frac{\text { in } / \text { cycle }}{(\mathrm{ksi} \sqrt{\text { in }})^{m_{1}}}$ \\
\hline$\gamma$ & 0.55 & $7 \%$ & Dimensionless \\
\hline
\end{tabular}

The value of $\beta$ is related to the geometry of the structure as shown in Figures 2.6 and 2.7, and can be expressed by the least square regression as follows:

Growth towards small rivet hole:

$$
\beta=15878.62 a^{4}-4895.55 a^{3}+562.01 a^{2}-29.75 a+2.39
$$

Growth from satellite rivet hole:

$$
\beta=128285.12 a^{4}-173837.85 a^{3}+88331.08 a^{2}-19957.33 a+1695.45
$$

A random number is included in (4.12) and (4.13) in order to account for the uncertainties in the $\beta$ factor. This random number is generated in order to calculate the $\beta$ value between the lower and upper limits.

\subsubsection{Forman Model}

The Forman equation, published in 1967, modifies the exponential crack propagation equation proposed by Paris [14] to account for discrepancies when a large variation in the data exits. These discrepancies are: 1) The layering of the data 
owing to the load ratio, $R$, and 2) the instability of the crack growth when the stressintensity factor approaches the $K_{\mathrm{C}}$ value for the material [65].

To account for these discrepancies, and assuming that current fracture mechanics theory remains valid, a correct crack-growth model should have the following criteria,

$$
\lim _{\max K \rightarrow K_{\mathrm{C}}} \frac{\mathrm{d} a}{\mathrm{~d} N}=\infty
$$

Additionally, making the substitution,

$$
\max K=\frac{\Delta K}{1-R}
$$

where $R$ is the mean ratio (of the minimum stress-intensity factor to the maximum stress-intensity factor.) A more general requirement for crack-growth rate is,

$$
\lim _{\Delta K \rightarrow(1-R) K_{\mathrm{C}}} \frac{\mathrm{d} a}{\mathrm{~d} N}=\infty
$$

Assuming, then, that a correct crack-growth equation has both an exponential form with singularity at $(1-R) K_{\mathrm{C}}-\Delta K$, an improved crack growth equation is proposed as

$$
\frac{\mathrm{d} a}{\mathrm{~d} N}=\frac{C_{2}(\Delta K)^{m_{2}}}{(1-R) K_{\mathrm{C}}-\Delta K}
$$

By substituting the stress intensity factor, $\Delta K$, in terms of stress and crack size, (4.17) may re-written as

$$
\frac{\mathrm{d} a}{\mathrm{~d} N}=\frac{C_{2}(\beta \Delta S \sqrt{\pi a})^{m_{2}}}{(1-R) K_{\mathrm{C}}-\Delta K}
$$


where $C_{2}$ and $m_{2}$ are material constants. It is important to note that the parameter constants, $m$ and $C$, in the Walker equation, Forman equation, closure, and Paris equation do not have the same numerical values or units [66].

Figure 4.4 shows a comparison of equation 4.17 and the test results for 7075T6. The wide range of test data in the plots show the correlation for the layering effects at different $R$-values, and for the predicted theoretical asymptote. The material constants used for calculating the theoretical curves in Figure 4.4 are, $K_{\mathrm{C}}=68,000$ $1 \mathrm{~b} / \mathrm{in}^{1 / 2}, C_{2}=5 \mathrm{E}-13$, and $m_{2}=3$.

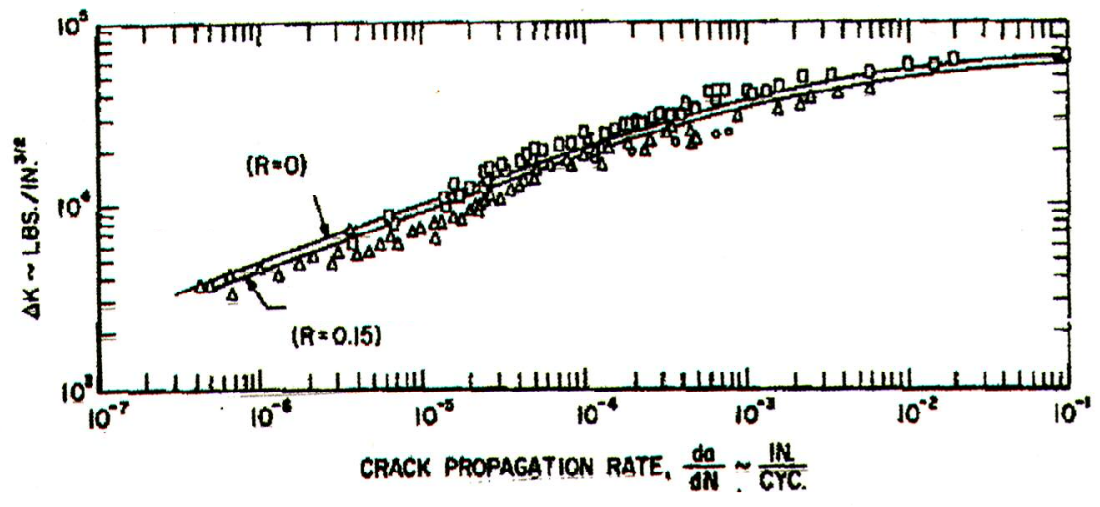

Figure 4.4 Comparison of experimental and theoretical crack-propagation rates in 7075-T6 aluminum plate for $R=0$ to $R=0.15$ [65].

Parameters $m_{2}, C_{2}$ and $K_{\mathrm{C}}$ [38], are sampled from normal distributions with the characteristics described in Table 4.2. The value of $\beta$ is related to geometry of the structure as shown in Figures 2.6, and 2.7. 
Table 4.2 Forman Parameters.

\begin{tabular}{|c|c|c|c|}
\hline Parameter & Mean & $\begin{array}{c}\text { Coefficient of } \\
\text { variation }\end{array}$ & Units. \\
\hline$m_{2}$ & 3.21 & $5 \%$ & Dimensionless \\
\hline$C_{2}$ & $2.56 \mathrm{E}-07$ & $7 \%$ & $\frac{\text { in } / \text { cycle }}{(\mathrm{ksi} \sqrt{\text { in }})^{m_{2}-1}}$ \\
\hline$K_{\mathrm{C}}$ & 70 & $7 \%$ & $(\mathrm{ksi} \sqrt{\mathrm{in}})$ \\
\hline
\end{tabular}

\subsection{Interaction model: Closure Model}

Experiments $[67,68]$ on metals have shown that fatigue cracks remain closed during part of the load cycle under constant and variable-amplitude loading. This phenomenon is known as the crack-closure concept. A model that account for crack closure can be used to calculate crack growth under constant-amplitude loading [69], and is a significant factor in causing load-interaction effects on crack growth rates and retardation/acceleration, under variable-amplitude loading [67]. Crack-closure is caused by the presence of a plastic zone ahead of a crack tip, which is generally accepted as a characteristic of crack behavior in aluminum alloys [37]. The material adjacent to the crack surfaces near a crack tip in the plastic zone become deformed in tension. Consequently, as the load is reduced, this material forces the crack surfaces to close. Figure 4.5 illustrates the crack closure concept. 


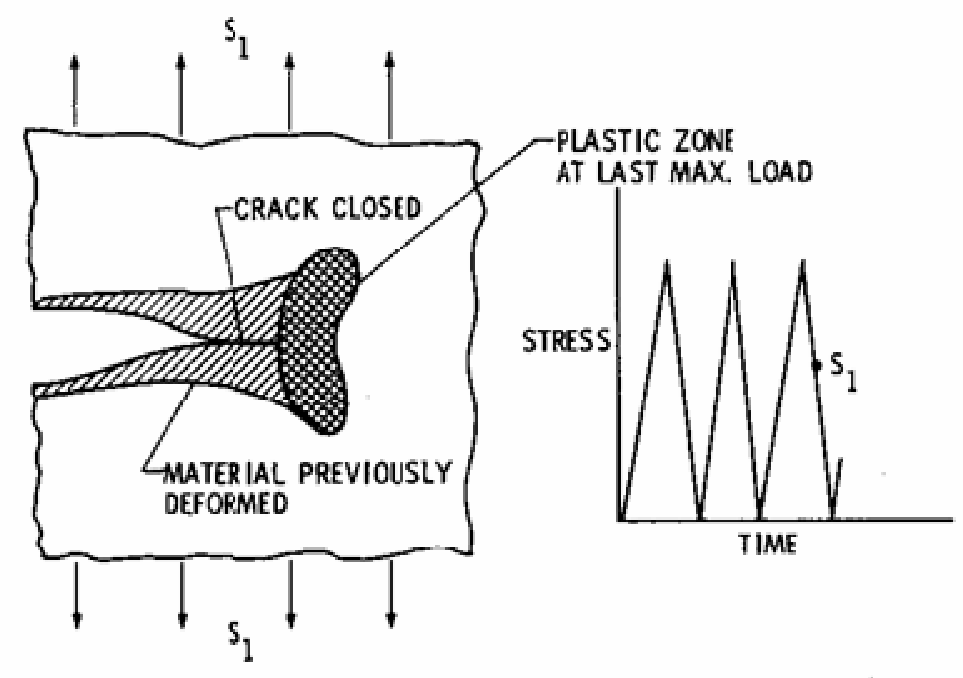

Figure 4.5 Crack closure concept [37].

Crack propagation can only occur during that portion of the loading cycle in which the crack is fully open at the crack tip; therefore, the crack opening stress is used as a reference stress level from which an effective stress range is obtained. The effective stress range is defined as

$$
\Delta S_{\text {eff }}=S_{\text {max }}-S_{\text {op }}
$$

where $S_{\mathrm{op}}$ is the crack opening stress. An effective stress ratio is then defined as [69]:

$$
U=\frac{\left(S_{\max }-S_{\mathrm{op}}\right)}{\left(S_{\max }-S_{\min }\right)}=\frac{\Delta S_{\text {eff }}}{\Delta S}=\frac{\Delta K_{\text {eff }}}{\Delta K}
$$

Constant amplitude loading tests were conducted to establish the relationship between $U$ and three variables which were anticipated to have a significant effect on $U$ (stress intensity range, crack length, and stress ratio). For the given range of testing conditions $R$ is the significant variable. Empirical relations for some aluminum alloys are as follows $[69,70]$ :

- 2024-T3, $U=0.5+0.4 R(-0.1<R<0.7)$ 
- $7175-\mathrm{T} 651, U=0.4+0.4 R[71]$

- $7475-\mathrm{T} 73, U=0.618+0.365 R+0.139 R^{2}[72]$

- $\quad$ mild steel, $U=1 /(1.5-R)[73]$

Measurements of crack opening stresses are very complex and have been taken on only a few materials and for a limited number of loading variables. In this research, the crack closure phenomenon was analyzed using the state-space model proposed by Ray and Patankar $[74,75]$. This model is structurally similar to the Paris Equation [14], which was modified for crack closure, and subsequently used in the FASTRAN code [27]. The major difference is in the formulation of transient behavior of the crack opening stress. The crack opening stress in FASTRAN is calculated asynchronously based on a relatively long history of stress excitation over the past $(\sim 300)$ cycles, while the state-space model captures the effects of stress overload and reverse plastic flow, and is applicable to various types of loading including single-cycle overloads, irregular sequences and random loads [75]. It is important to note that when the model predictions of using the state-space model were compared with those of FASTRAN and AFGROW codes for identical input stress excitation, results were close [75].

The FASTRAN model is based on the equation for the effective stressintensity factor range given by [76],

$$
\Delta K_{\text {eff }}=\left[\left(1-S_{0 \mathrm{p}} / S_{\max }\right) /(1-R)\right] \Delta K
$$

where

$$
S_{0 \mathrm{p}} / S_{\text {max }}=A_{0}+A_{1} R+A_{2} R^{2}+A_{3} R^{3} \quad \text { for } R \geq 0
$$

and 


$$
S_{0 \mathrm{p}} / S_{\max }=A_{0}+A_{1} R \quad \text { for }-1 \leq R<0
$$

when $S_{0 \mathrm{p}} \geq S_{\min }$. The coefficients are defined by

$$
\begin{aligned}
& A_{0}=\left(0.825-0.34 \alpha+0.05 \alpha^{2}\right)\left[\cos \left(\frac{\pi}{2} \frac{S_{\text {max }}}{S_{\text {flow }}}\right)\right]^{1 / \alpha} \\
& A_{1}=(0.415-0.071 \alpha)\left(\frac{S_{\text {max }}}{S_{\text {flow }}}\right) \\
& A_{2}=\left(1-A_{0}-A_{1}-A_{3}\right) \\
& A_{3}=\left(2 A_{0}+A_{1}-1\right) \\
& S_{\text {flow }}=\frac{S_{y}+S_{\text {ult }}}{2}
\end{aligned}
$$

where $R$ is the stress ratio, $S_{y}$ is the yield strength, $S_{u l t}$ is the ultimate strength, and the constants $A_{0}, A_{1}, A_{2}, A_{3}$ are functions of the stress ratio, the stress level, $S_{\text {flow }}$ the flow stress, and the constraint factor $\alpha$.

The state-space model states that the information for calculating the memorydependent variable in damage crack growth can be modeled in a finite-dimensional state-space setting by an ordinary differential equation, and is formulated based on the crack closure concept, where the crack length and the crack opening stress are the state variables. It is a modification of the Paris equation defined earlier in section 2.3, in which the inputs are $S_{k}^{\max }$ and $S_{k}^{\min }$ in the $k_{\text {th }}$ cycle and the output is the crack length increment $\Delta a_{k}$.

By expressing the dynamic behavior of damage crack growth as a derivative $\mathrm{d} a / \mathrm{d} N$ with respect to the number of cycles, i.e., $\Delta a_{k}$ in the $k_{\mathrm{th}}$ cycle as, 


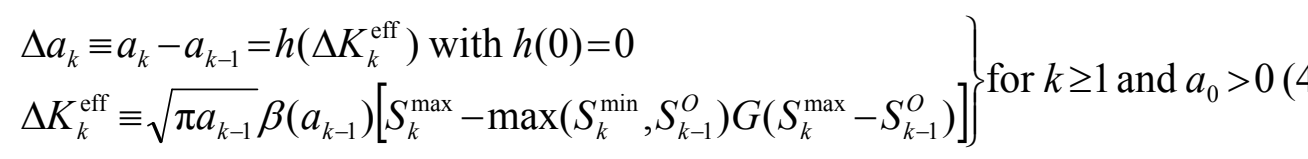

where $a_{k-1}$ and $S_{k-1}^{O}$ are the crack length and the crack-opening stress, respectively, during the $k_{\text {th }}$ cycle and change to $a_{k}$ and $S_{k}^{O}$ at the end of the $k_{\text {th }}$ cycle; $\beta(\cdot, \cdot)$ is related to geometry of the structure as shown in Figures 2.6 and 2.7, and $G(x)$ is the Heaviside function, defined by

$$
G(x)=\left\{\begin{array}{l}
0 \text { if } x<0 \\
1 \text { if } x \geq 0
\end{array}\right.
$$

The non-negative monotonically increasing function $h(\cdot)$ can be represented either by a closed form algebraic equation or by lookup table [27];

In this research, the following lookup table is used to represent the nonnegative function $h\left(\Delta K_{k}^{\text {eff }}\right)$ in Equation 4.29 for the aluminum 7075-T6,

Table 4.3 Crack growth lookup table for 7076-T6 [75].

\begin{tabular}{|c|c|c|c|}
\hline $\begin{array}{c}\Delta \mathrm{K}_{\text {eff }} \\
{\left[{\left.\mathrm{Mpa}-\mathrm{m}^{1 / 2}\right]}^{1 / 2}\right.}\end{array}$ & $\begin{array}{c}\mathrm{d} a / \mathrm{d} N \\
{[\mathrm{~m} / \text { cycle }]}\end{array}$ & $\begin{array}{c}\Delta \mathrm{K}_{\text {eff }} \\
{\left[\mathrm{Ksi}_{-1}{ }^{1 / 2}\right]}\end{array}$ & $\begin{array}{c}\mathrm{d} a / \mathrm{d} N \\
{[\mathrm{in} / \text { cycle }]}\end{array}$ \\
\hline 0.90 & $1.00 \mathrm{E}-11$ & 0.82 & $3.94 \mathrm{E}-10$ \\
\hline 1.35 & $1.20 \mathrm{E}-09$ & 1.23 & $4.72 \mathrm{E}-08$ \\
\hline 3.40 & $1.00 \mathrm{E}-08$ & 3.09 & $3.94 \mathrm{E}-07$ \\
\hline 5.20 & $1.00 \mathrm{E}-07$ & 4.73 & $3.94 \mathrm{E}-06$ \\
\hline 11.90 & $1.00 \mathrm{E}-06$ & 10.83 & $3.94 \mathrm{E}-05$ \\
\hline 18.80 & $1.00 \mathrm{E}-05$ & 17.11 & $3.94 \mathrm{E}-04$ \\
\hline 29.00 & $1.00 \mathrm{E}-04$ & 26.39 & $3.94 \mathrm{E}-03$ \\
\hline
\end{tabular}

The rest of the formulation for the state-space model can be found in references $[74,75]$

Parameters $S_{y}, S_{u l t}$ and $E$ [38], are sampled from normal distributions with the characteristics described in Table 4.4. 
Table 4.4 Materials properties for the 7057-T6.

\begin{tabular}{|c|c|c|c|}
\hline Parameter & Mean & $\begin{array}{c}\text { Coefficient of } \\
\text { variation }\end{array}$ & Units. \\
\hline$S_{y}$ & 72 & $7 \%$ & $\mathrm{ksi}$ \\
\hline$S_{\text {ult }}$ & 85 & $5 \%$ & $\mathrm{ksi}$ \\
\hline$E$ & $1.00 \mathrm{E}+04$ & $5 \%$ & $\mathrm{ksi}$ \\
\hline
\end{tabular}

In the preceding section the process of combining material and parameters uncertainties through the damage crack growth models were discussed. The uncertainties and variabilities are accounted for using a Monte Carlo simulation of crack growth using a MatLab ${ }^{\circledR}$ routine, Probabilistic Crack GROWth (PCGROW), developed in this research.

\subsection{Failure Criteria}

Two failure criteria in the crack growth calculation can be seen in Figure 4.2. Half crack length greater than 1 inch or $K_{\max }$ greater than $K_{C}$ are determined by using Equation 2.6 and Figure 4.6 [31], respectively. For the structure analyzed in this research,

$$
B \geq 2.5\left(\frac{26 \mathrm{ksi} \sqrt{\mathrm{in}}}{72 \mathrm{ksi}}\right)^{2} \geq 0.300 \mathrm{in}
$$

The structural part of the aircraft under analysis is 0.080 inches thick. Therefore, not thick enough to meet the requirement of Equation 2.6. According to this, plane stress can better characterizes this condition than does plane strain, and the critical stress intensity factor, $K_{C}$, to be used as failure criteria is determined using Figure 4.6. 


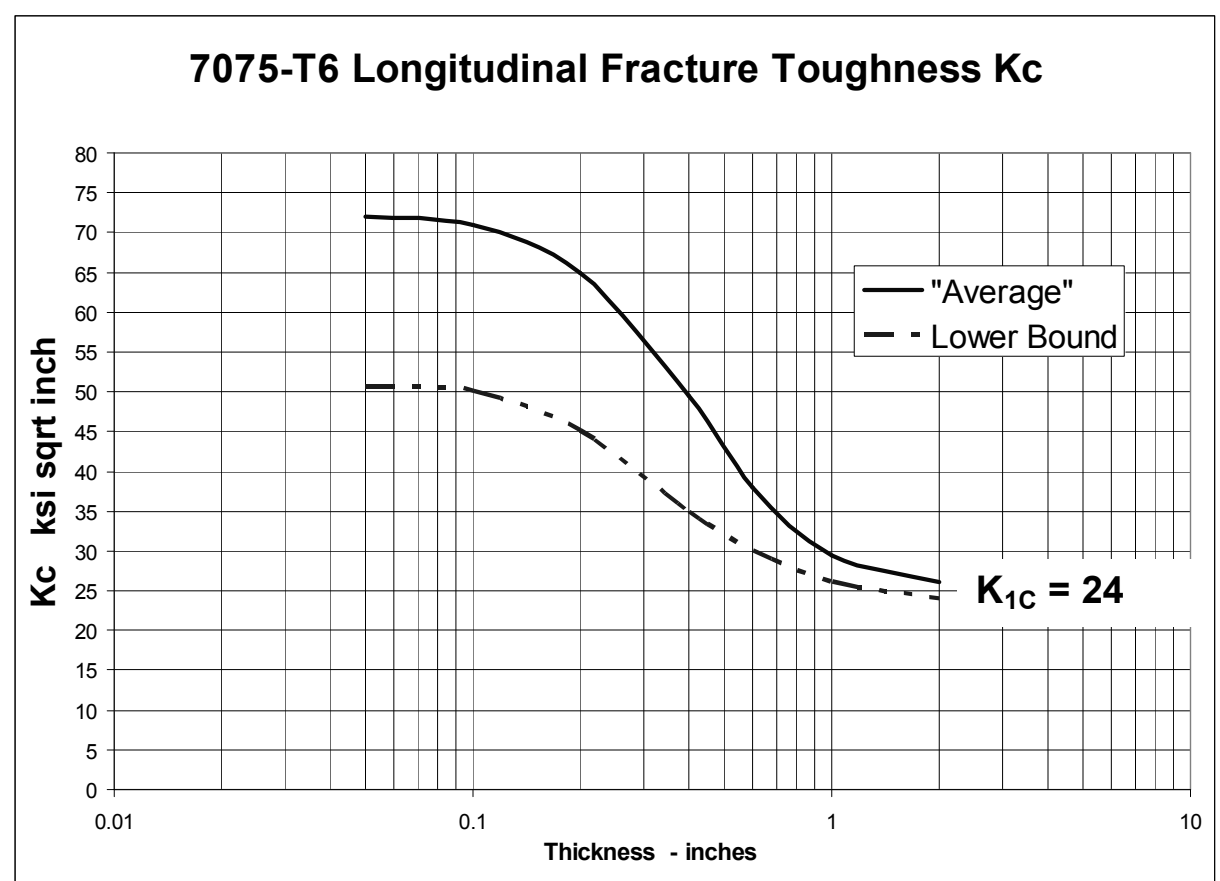

Figure 4.6 Stress intensity Factor for the 7075-T6 aluminum [31].

\subsection{Crack Growth Estimation and Uncertainty Propagation}

The damage crack growth estimation and uncertainty propagation from the input variables to the fatigue life is carried out by a simple Monte Carlo simulation. The procedure consists of four steps, shown in Figure 4.7. 


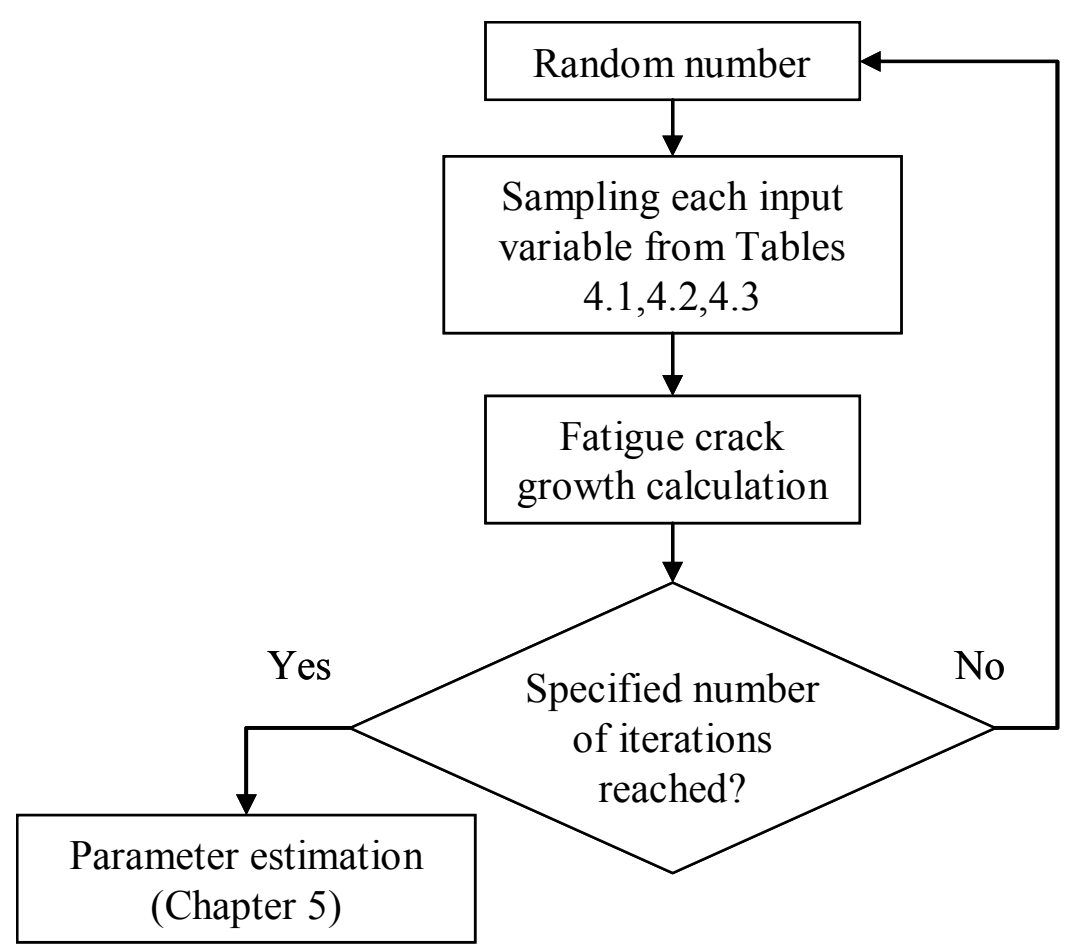

Figure 4.7 Crack growth and uncertainty propagation algorithm.

Figure 4.8 illustrates an example of the number of possible solutions when one considers the scatter of material properties and model parameters. About $50 \%$ of the crack sizes at this FLE are below 0.0172 ", and $75 \%$ are below 0.0215 ". 


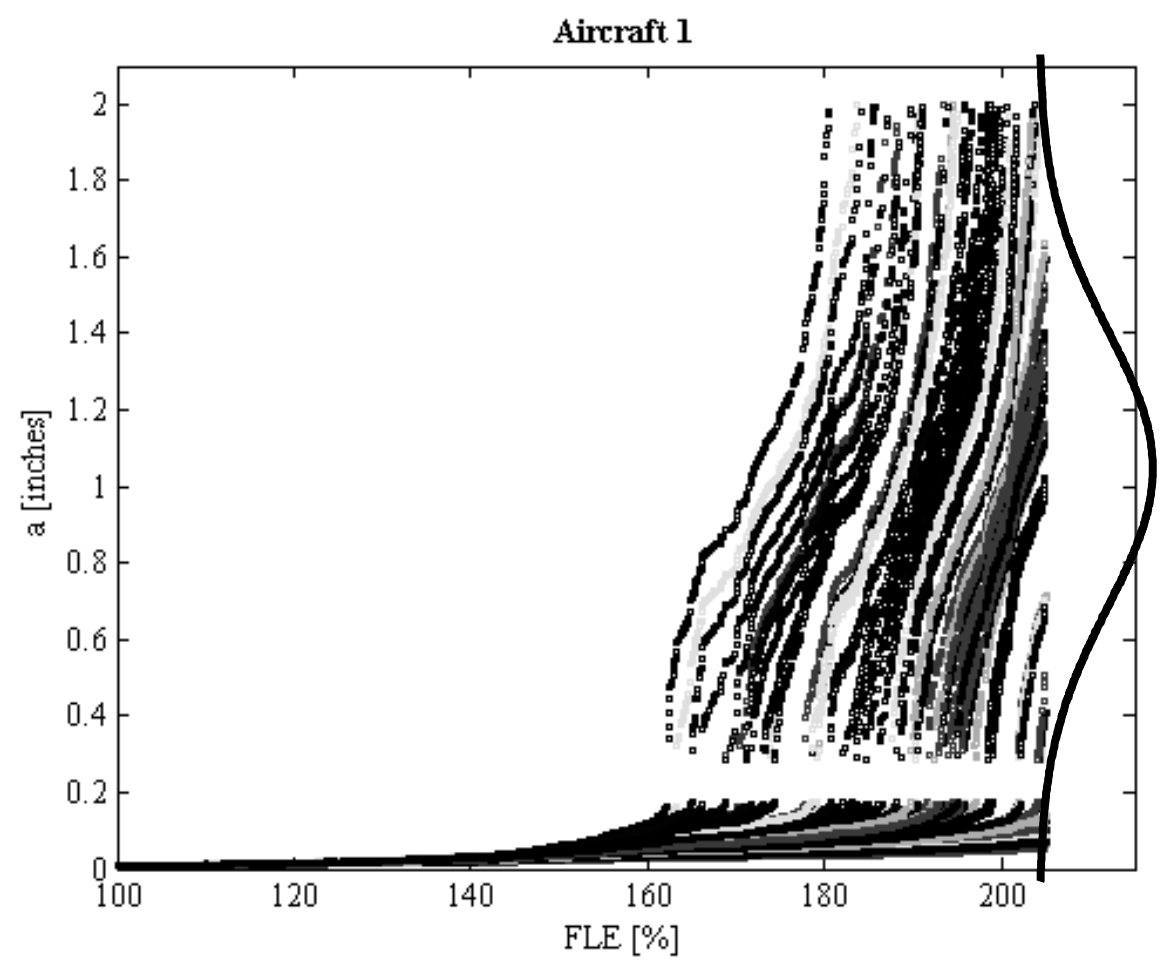

Figure 4.8 Example of Random Rack Growth trajectories at 231\% FLE.

\subsection{Model Verification}

This section validates the routines included in PCGROW by comparing different types of cycle loading with the predictions made by the models that are available in the AFGROW software package. AFGROW, developed by Harter [77], uses crack closure concept to determine $\Delta K_{\text {eff. }}$ In AFGROW, the opening stress intensity factor, $K_{\mathrm{op}}$, is determined by using a closure factor $\left(C_{f}\right)$ according to the following relationship [78],

$$
C_{f}=1-\left[\left(1-c_{f 0}\right)(1+0.6 R)(1-R)\right\rfloor
$$


where $C_{f 0}$ is the experimentally determined value of $C_{f}$ at $R=0$ and is assumed to be a material parameter.

AFGROW has an option to input the existing stress intensity factor solution in the form of a beta table for any 1-D crack, using the through crack geometry model, which is illustrated in Figure 4.9, where $w$ and $T$ are the respective width and thickness of the specimen. For the calculations, an initial crack length equal to $0.009^{\prime}$ ' is assumed.

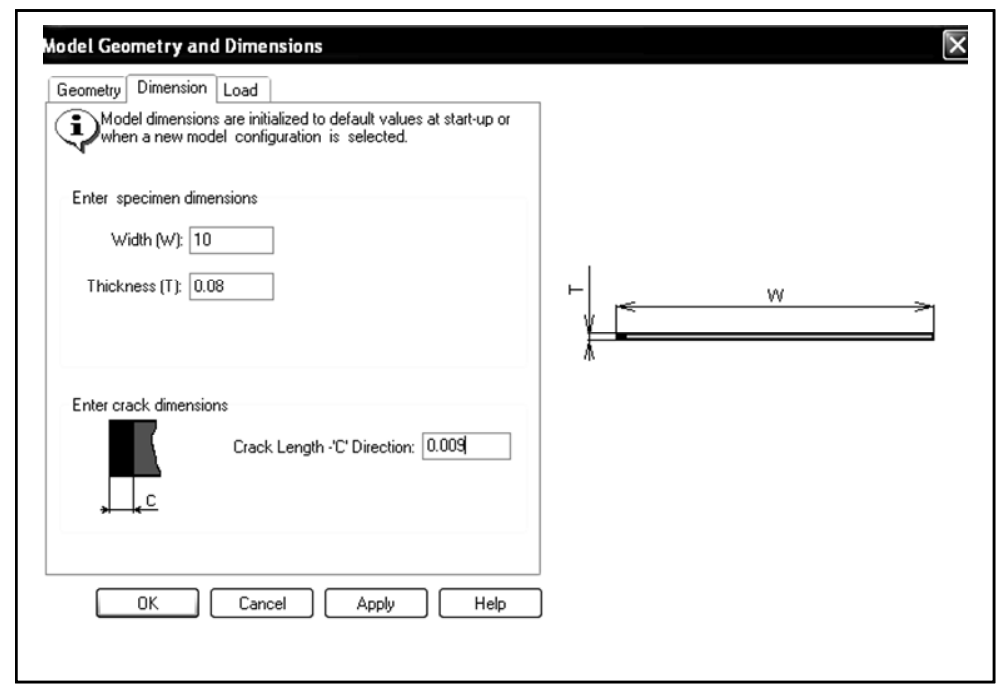

Figure 4.9 Model geometry and dimension in AFGROW.

Appropriate beta values are used at various crack lengths, so that the appropriate value at a given crack length may be interpolated. For the crack length dimension, we have,

$$
K=S \sqrt{\pi c} \beta(c)
$$

where $S$ is the stress level, $c$ is the crack length, and $\beta(c)$ is given by the following table, 
Table 4.5 Beta values.

\begin{tabular}{|c|c|}
\hline $\boldsymbol{c}$ [inches] & $\boldsymbol{\beta}$ \\
\hline 0.0000 & 2.486 \\
\hline 0.0007 & 2.237 \\
\hline 0.0130 & 2.178 \\
\hline 0.0260 & 1.929 \\
\hline 0.0390 & 1.772 \\
\hline 0.0650 & 1.779 \\
\hline 0.1040 & 1.770 \\
\hline 0.1300 & 1.769 \\
\hline 0.1500 & 2.100 \\
\hline 0.1740 & 3.000 \\
\hline
\end{tabular}

The material properties and empirical parameters for the 7075-T6 aluminun alloy are available in the library of AFGROW, under the module Nasgro equation.

Four different blocks loading spectrums were used, according to the profile shown in Figure 4.10, where $f$ and $s$, indicate that a block of $f$ constant amplitude cycles is followed by a block of $s$ different constant amplitude cycles. The values of $\mathrm{S}_{1}, \mathrm{~S}_{2}$, and $\mathrm{S}_{\min }$ were assumed in this study by considering the real values from the spectrum flight data.

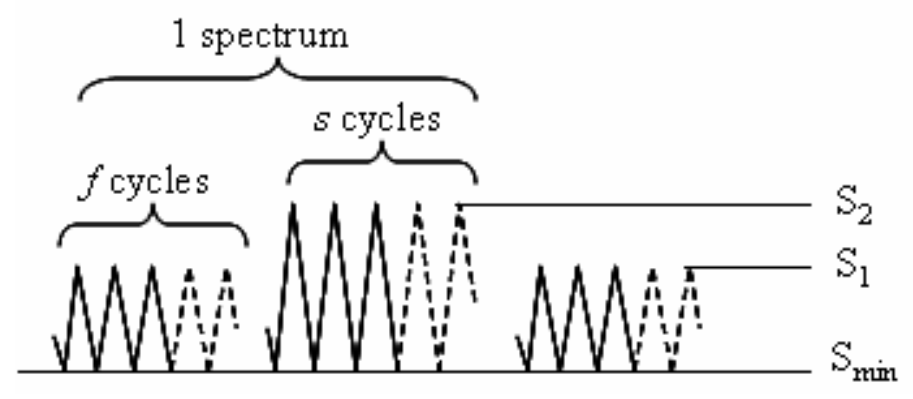

Figure 4.10 Spectrum profile. 
Figure 4.11 shows a comparison of PCGROW predictions with the predictions of AFGROW for the crack closure model. The results show that both codes produce similar results for case 1 under constant amplitude cyclic stresses. For cases 2 to 4 , under variable amplitude cyclic stresses, the results show that PCGROW predictions are close to the predictions of AFGROW. The difference is much due to the difference in computational algorithms used in those codes.
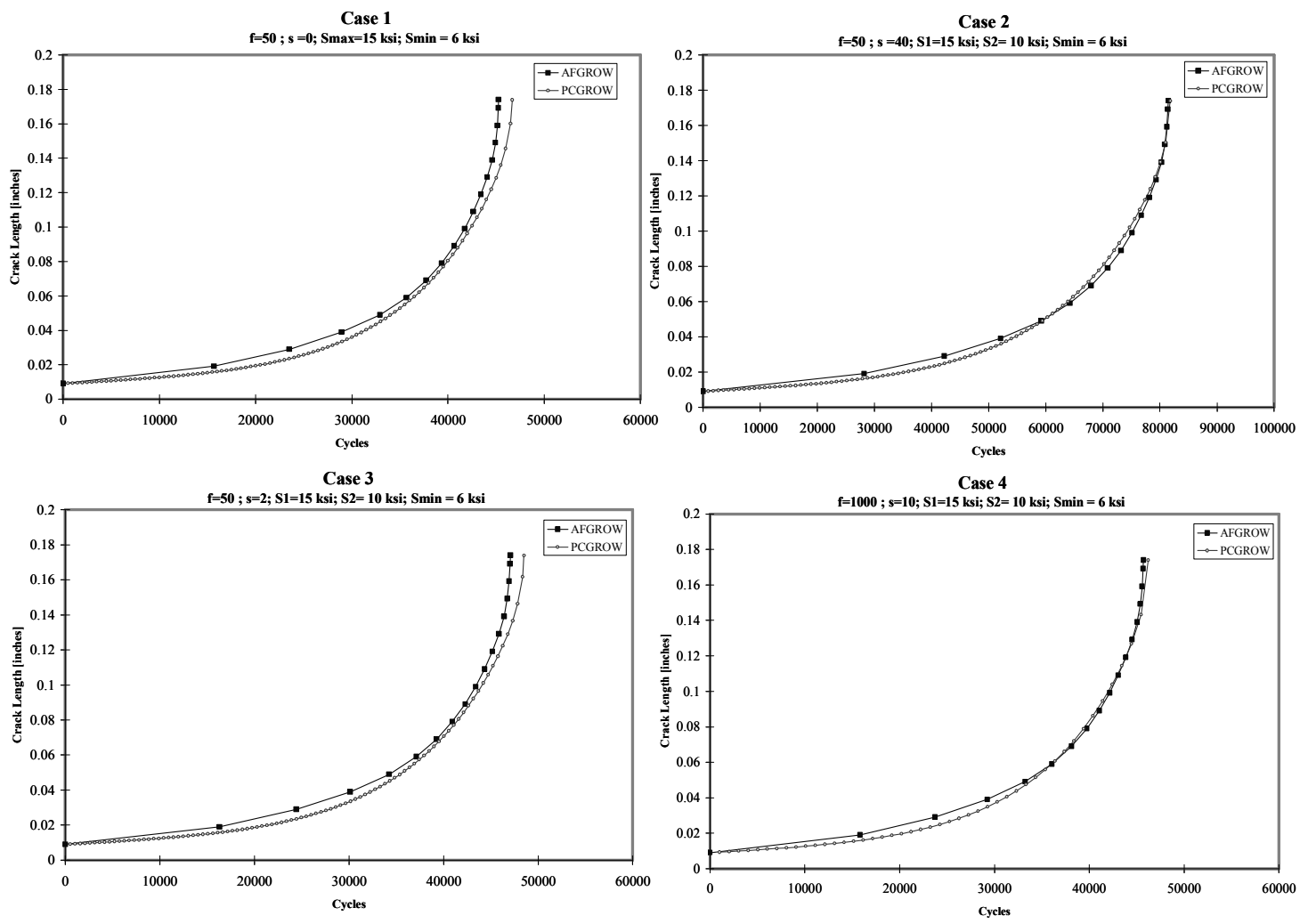

Figure 4.11 PCGROW and AFGROW comparison - Closure.

A fifth spectrum considering three overloads and one underload was also analyzed (see Figure 4.12), using the Walker, Forman and Crack Closure models. 


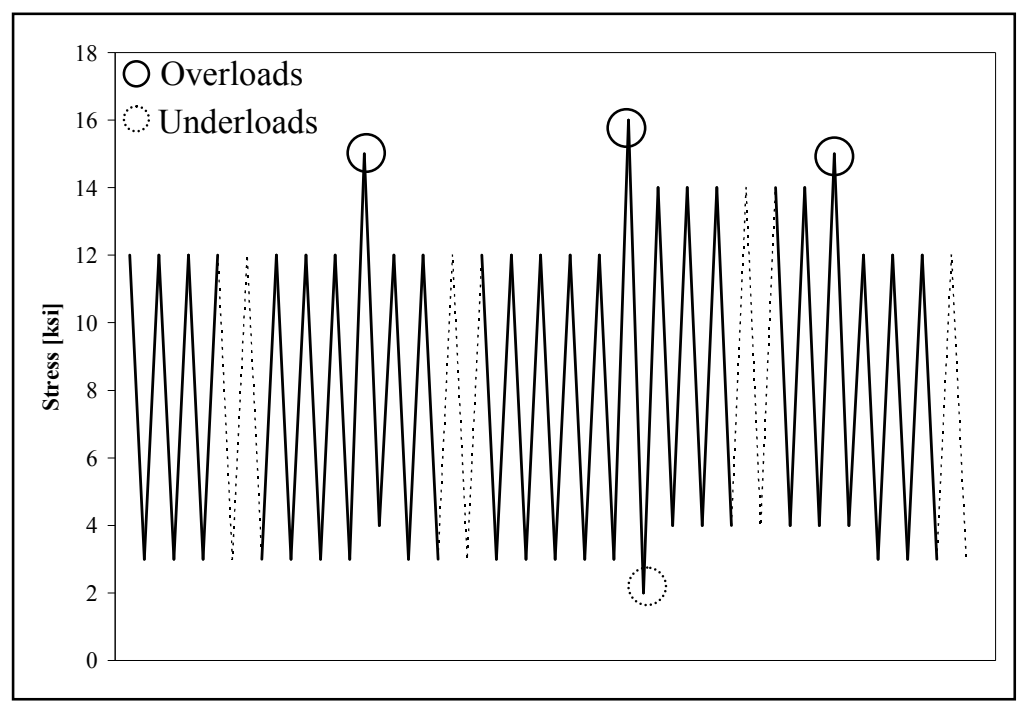

Figure 4.12 Overload and underload spectrum.

The plot in Figure 4.13 indicates that the accuracy of PCGROW is comparable to the AFGROW predictions for overload and underload amplitudes. The result from PCGROW is slightly more conservative than AFGROW.

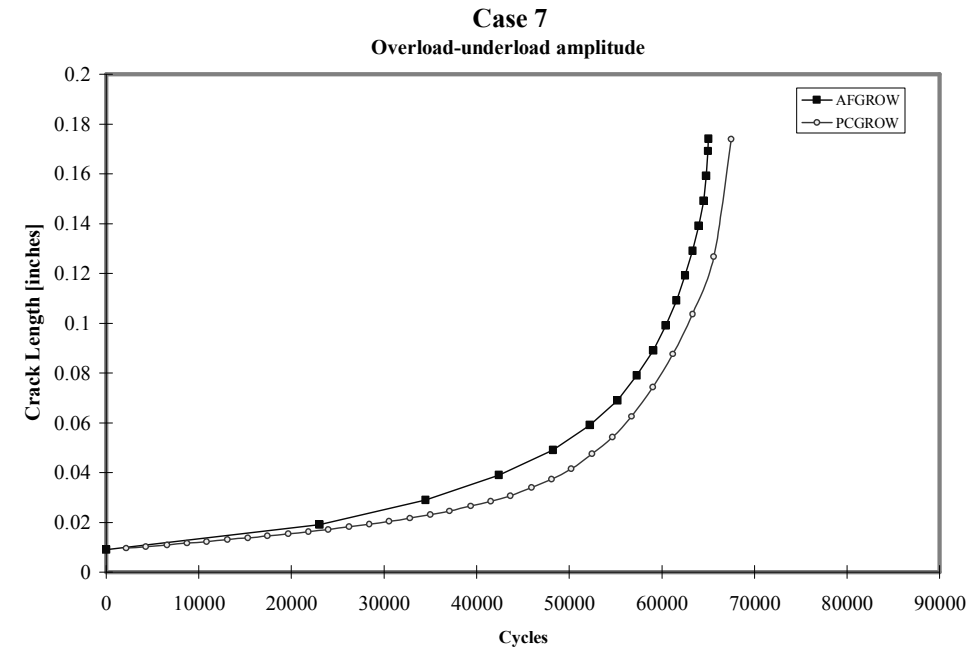

Figure 4.13 Results under overload-underload amplitude. 
The same analysis was carried out for the routines developed for noretardation effect, Walker and Forman models, and the results shown in Figures 4.14 and 4.15 indicate that PCGROW predictions are very close to the predictions obtained from AFGROW.
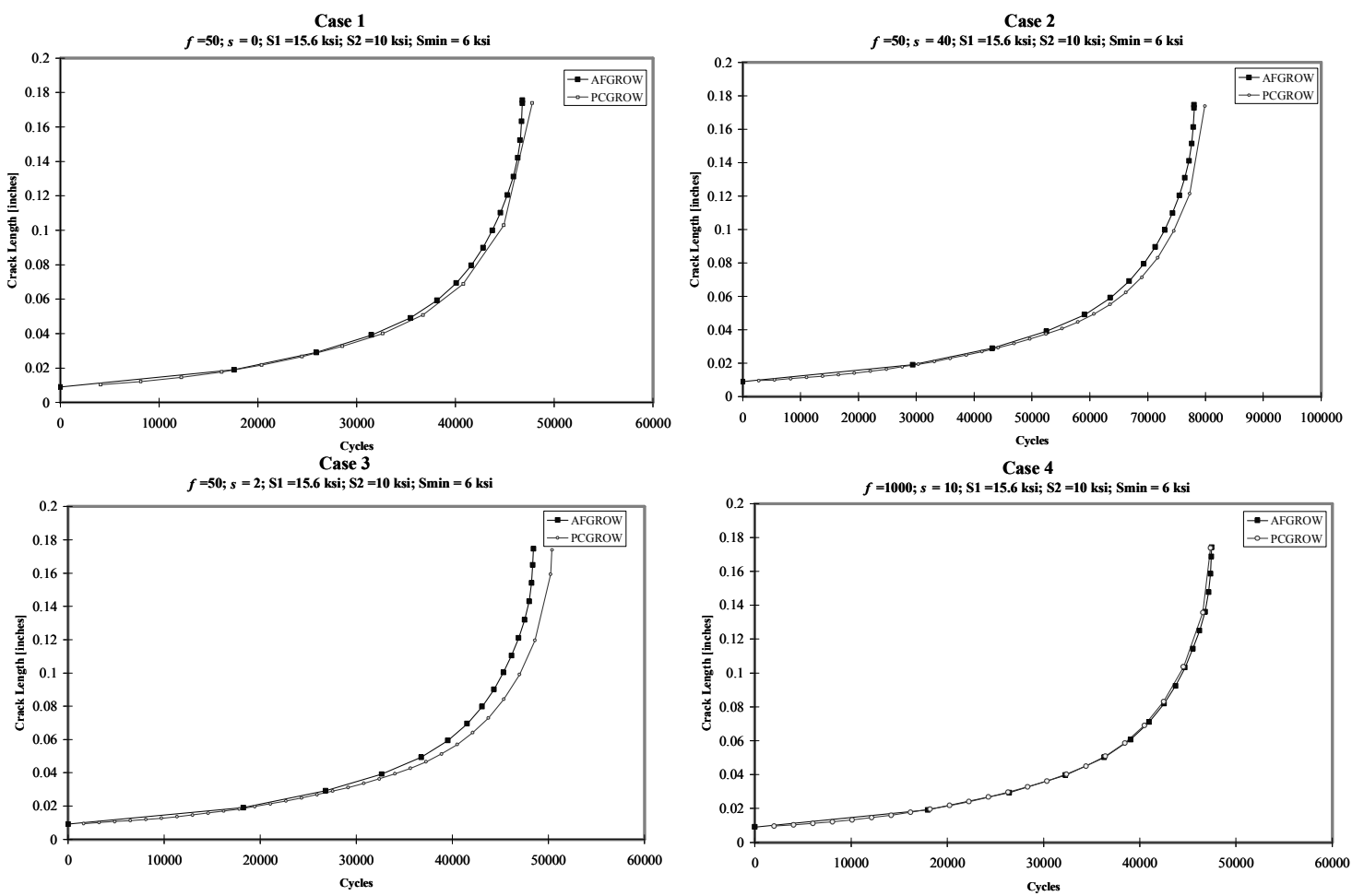

Figure 4.14 PCGROW and AFGROW comparison - Walker. 

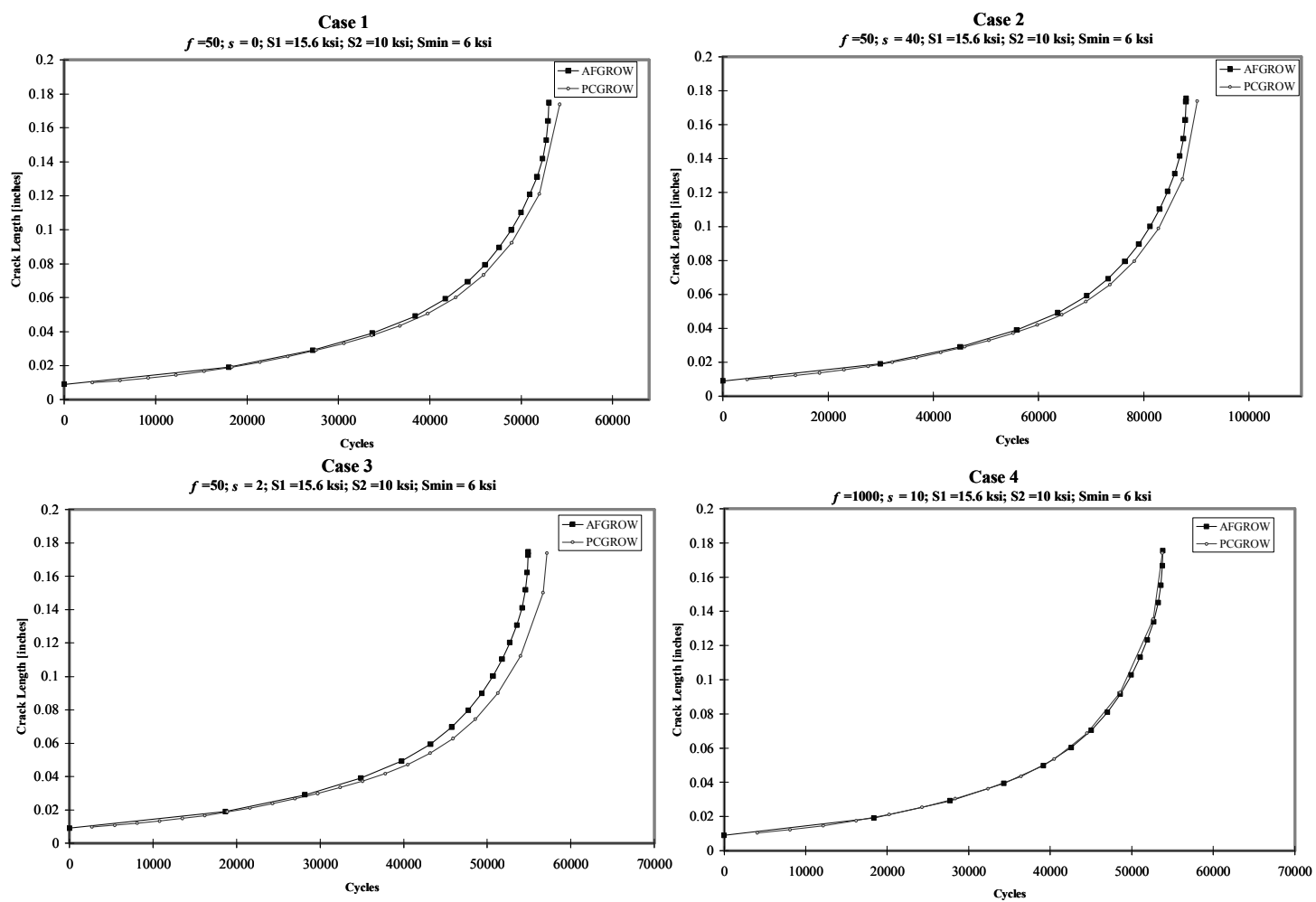

Figure 4.15 PCGROW and AFGROW comparison - Forman.

Generally in the deterministic validation effort it can be concluded that for the block and overload-underload cycling amplitudes, accuracy of PCGROW are comparable to the results obtained from the AFGROW code. Therefore, it can be concluded that the results from the models used is this study are acceptable for further use in probabilistic analysis. 


\section{Chapter 5 Probabilistic Parameter Estimation of the}

\section{Models.}

The objective of this research is to analyze the behavior of the damage crack growth under random loads and by considering material variability (aleatory uncertainty) and by considering modeling uncertainty (epistemic uncertainty). For useful statistical inference, we are proposing a lifetime model, which consists of an underlying life distribution that describes the structure at different stress levels and a stress-life relationship that quantifies the manner in which the life distribution changes as a function of stress levels [79]. Figure 5.1 shows the elements of this approach.
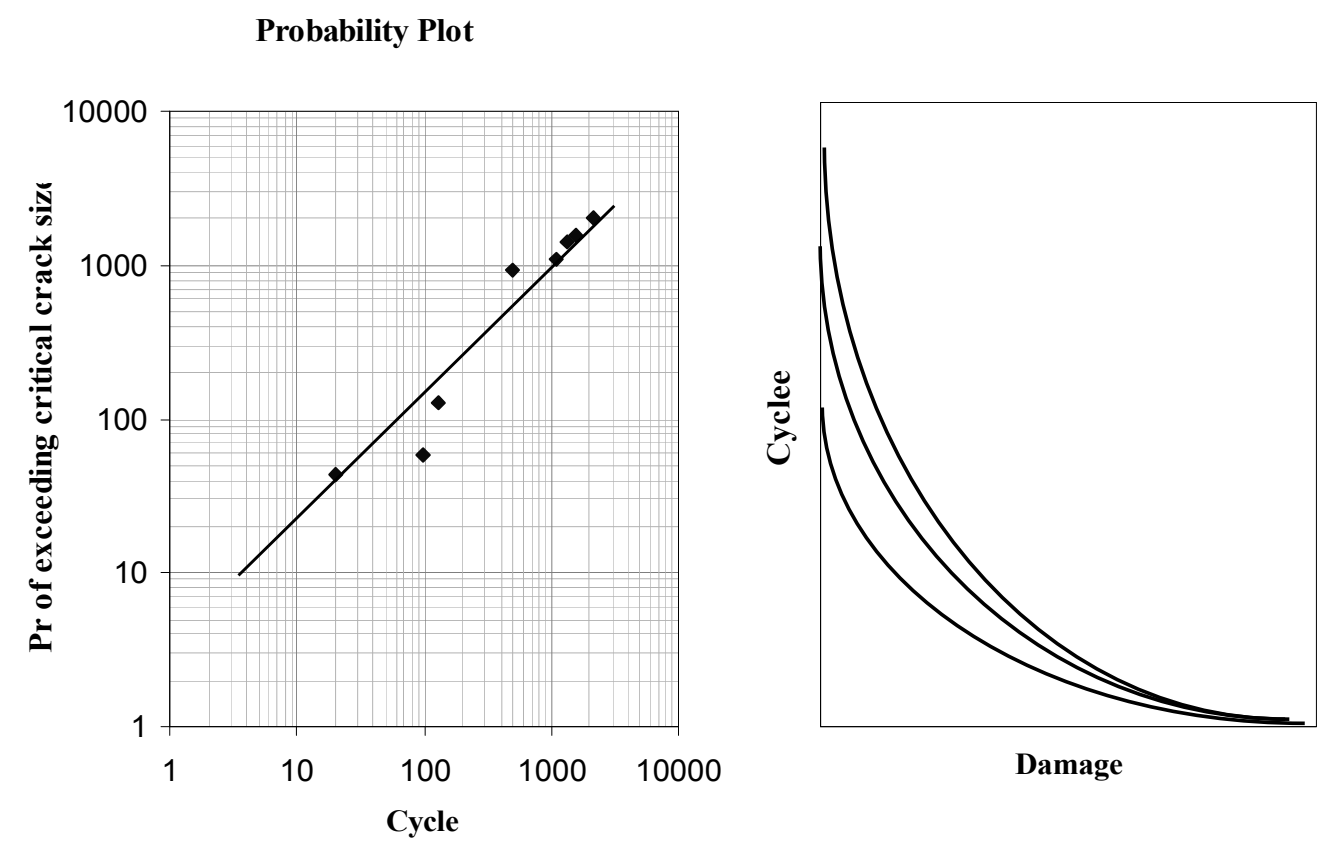

Figure 5.1 Underlying life distribution and stress-life relationship at different stress levels. 
The combination of both the life distribution and the stress-life model can be best seen in Figure 5.2 where a joint $p d f$ is plotted against both time and stress.

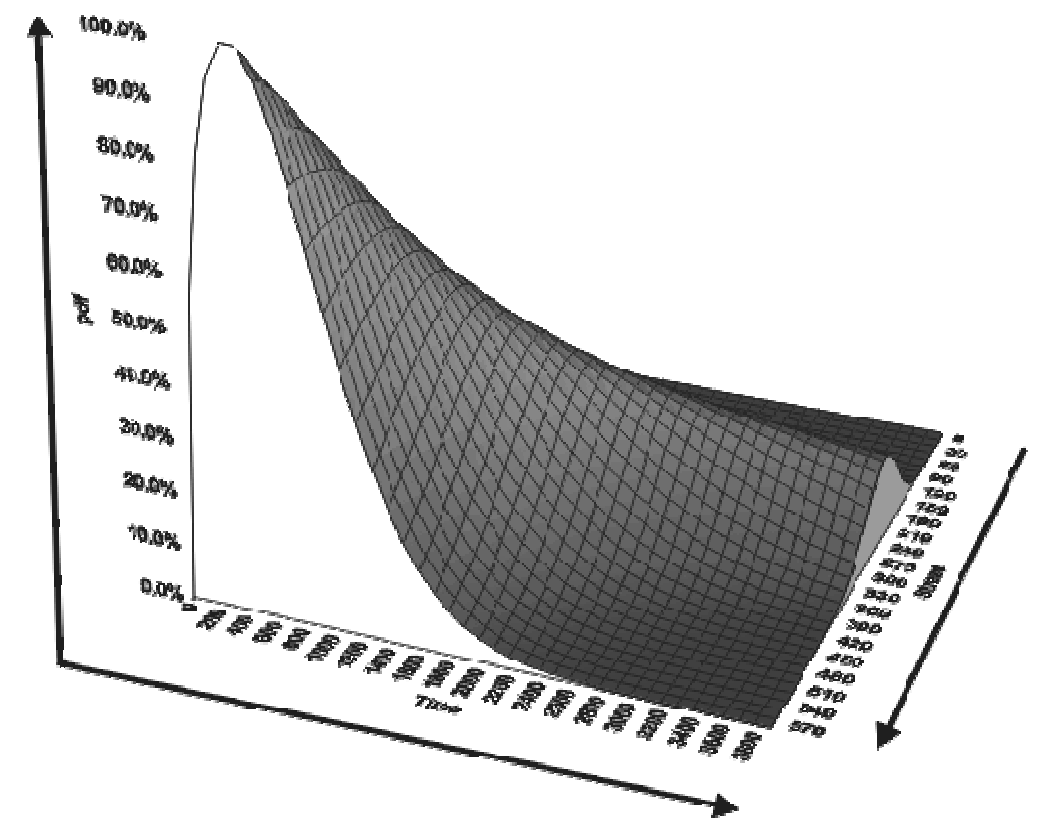

Figure $5.2 p d f$ vs. time and stress. [80].

The assumed underlying life cycle to failure distribution can be any distribution form, and the stress or damage life relationship can be any empirically derived relationships consistent with underlying physical principles describing degradation or damage. The objective then becomes to obtain the parameters of the life distribution and the life-stress damage relationship [80].

This research deals with the probability of structural failure due to fatigue damage (i.e. fracture mechanics). It models damage crack growth by assuming a lognormal life distribution, and using the empirical Inverse Power Law (IPL) to 
depict the form of damage median life relationship. Lognormal distribution and IPL relationships have shown to best fit the crack size (damage) and FLE (life expended). In the following paragraphs, we will explain the theory that supports this assumption.

\subsection{Lognormal Distribution as a Representation of Crack Size}

\section{Distribution.}

One of the commonly used crack size distribution in fatigue is the lognormal distribution [81]. This distribution is particularly appropriate when the cycle to failure or repair has cumulative contributing factors. This property can be seen in several degradation processes associated with fatigue and creep failure mechanisms. The degradation in such cases is generally progressive. For example, a crack grows rapidly under high stress, because the stress intensity increases progressively (damage accumulation) as the crack grows [82]. The basic properties of lognormal distribution were established long ago (Weber [83], Fechner [84], Galton [85], McAlister [86]), and it is not difficult to characterize lognormal distributions mathematically. A random variable, $\boldsymbol{X}$, is said to be $\operatorname{lognormally}$ distributed if $\log (\boldsymbol{X})$ (i.e. natural or based-10 logarithm) is normally distributed. Only positive values are possible for the variable, and the distribution is skewed to the left. Two parameters are needed to specify a lognormal distribution. Traditionally, the mean $\mu$ and the standard deviation $\sigma\left(\right.$ or the variance $\left.\sigma^{2}\right)$ of $\log (\boldsymbol{X})$ are used [87].

In the fatigue material field, Sinclair and Dolan [2] have shown that lognormal distribution is a good fit to model fatigue life. Their experiment consisted of testing 
174 identical, highly polished, unnotched, 7075 aluminum specimens under six different stress levels. Figures 5.3 and 5.4 show the results obtained.

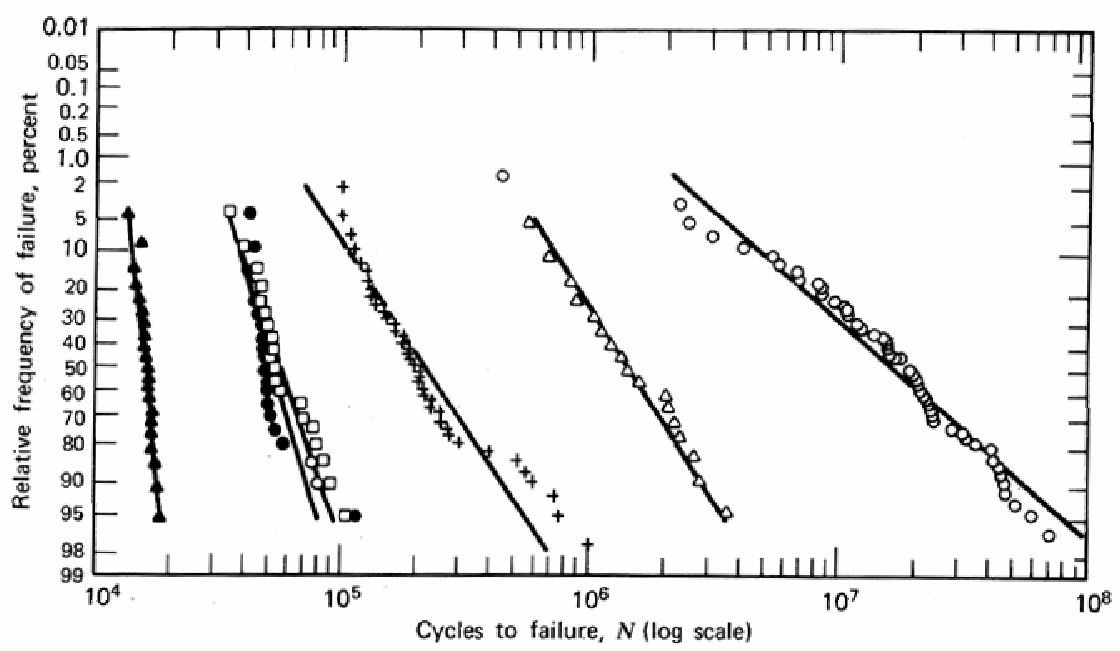

Figure 5.3 Lognormal fit for 7075 aluminum specimens under six different stress levels [2].

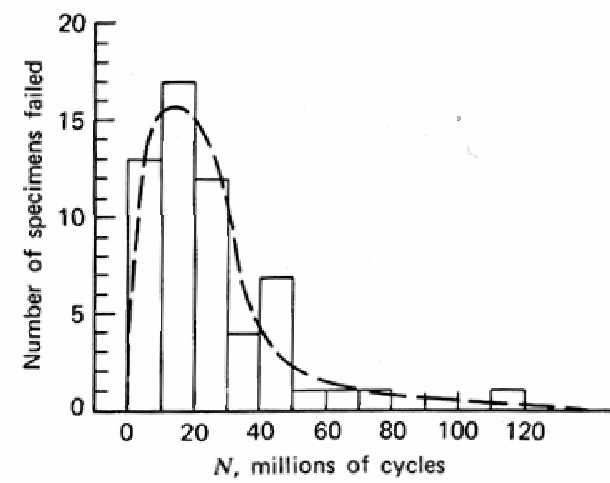

(a)

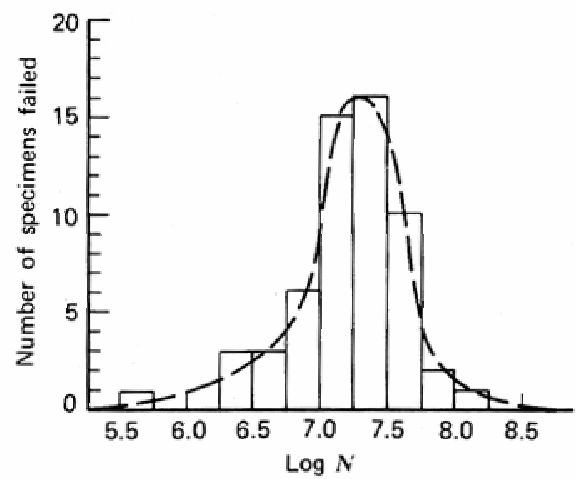

(b)

Figure 5.4 Lognormal fit for 7075 aluminum specimens [2].

In this study, to determine appropriateness of lognormal distribution, crack size data obtained from the Monte Carlo simulation at different $F L E$ 's were analyzed using two statistical goodness of fit tests: Chi-Square and Anderson-Darling at 0.05 
confidence level $[88,89]$. These statistics measure how good the distribution fits the input data and how confident one can be that the data was produced by the distribution function. For each of these statistics, the smaller the value, the better the fit. In this case, any fit that has a test value above the critical value is rejected, while fits with tests values below the critical value are accepted. The critical value for any hypothesis test depends on the significance level at which the test is carried out.

Table 5.1 shows that the best fit is the lognormal distribution, followed by the normal distribution. Weibull distribution does not show good fit at all.

Table 5.1 Goodness of fit results.

\begin{tabular}{|c|c|c|c|c|c|c|c|}
\hline \multirow[b]{2}{*}{$F L E$} & \multirow[b]{2}{*}{ Statistics } & \multicolumn{2}{|c|}{ Lognormal } & \multicolumn{2}{|c|}{ Normal } & \multicolumn{2}{|c|}{ Weibull } \\
\hline & & Chi-Sq & $\begin{array}{l}\text { Anderson- } \\
\text { Darling }\end{array}$ & Chi-Sq & $\begin{array}{l}\text { Anderson } \\
\text {-Darling }\end{array}$ & Chi-Sq & $\begin{array}{c}\text { Anderson- } \\
\text { Darling }\end{array}$ \\
\hline \multirow[b]{2}{*}{100} & Test Value & 11.600 & 0.246 & 18.320 & 0.499 & 16.240 & 0.768 \\
\hline & $\begin{array}{c}\text { C.Val @ } \\
0.05\end{array}$ & 35.173 & 0.752 & 35.173 & 0.751 & 35.173 & 0.757 \\
\hline \multirow[b]{2}{*}{112} & Test Value & 25.600 & 0.238 & 24.480 & 0.559 & 17.840 & 0.520 \\
\hline & $\begin{array}{c}\text { C.Val @ } \\
0.05\end{array}$ & 35.173 & 0.752 & 35.173 & 0.751 & 35.173 & 0.757 \\
\hline \multirow[b]{2}{*}{139} & Test Value & 18.160 & 1.109 & 44.880 & 1.898 & 18.240 & 0.495 \\
\hline & $\begin{array}{c}\text { C.Val@ } \\
0.05\end{array}$ & 35.173 & 0.752 & 35.173 & 0.751 & 35.173 & 0.757 \\
\hline \multirow[b]{2}{*}{152} & Test Value & 2.000 & 0.275 & 2.000 & 0.264 & 2.907 & 0.167 \\
\hline & $\begin{array}{c}\text { C.Val@ } \\
0.05\end{array}$ & 18.307 & 0.752 & 18.307 & 0.746 & 18.307 & 0.757 \\
\hline \multirow[b]{2}{*}{182} & Test Value & 5.856 & 0.219 & 11.300 & 0.747 & 6.082 & 0.227 \\
\hline & $\begin{array}{c}\text { C.Val @ } \\
0.05\end{array}$ & 18.307 & 0.752 & 18.307 & 0.746 & 18.307 & 0.757 \\
\hline \multirow[b]{2}{*}{213} & Test Value & 10.000 & 0.297 & 6.211 & 0.746 & 13.370 & 0.308 \\
\hline & $\begin{array}{c}\text { C.Val @ } \\
0.05\end{array}$ & 14.067 & 0.752 & 14.067 & 0.736 & 14.067 & 0.757 \\
\hline \multirow[b]{2}{*}{232} & Test Value & 4.000 & 0.344 & 5.600 & 0.725 & 4.400 & 0.334 \\
\hline & $\begin{array}{c}\text { C.Val@ } \\
0.05\end{array}$ & 14.067 & 0.752 & 14.067 & 0.737 & 14.067 & 0.757 \\
\hline
\end{tabular}


Moreover, one set from the above data is plotted on probability papers to confirm the results obtained from the goodness of fit tests. Figure 5.5 confirms that lognormal distribution is a good fit for this dataset.
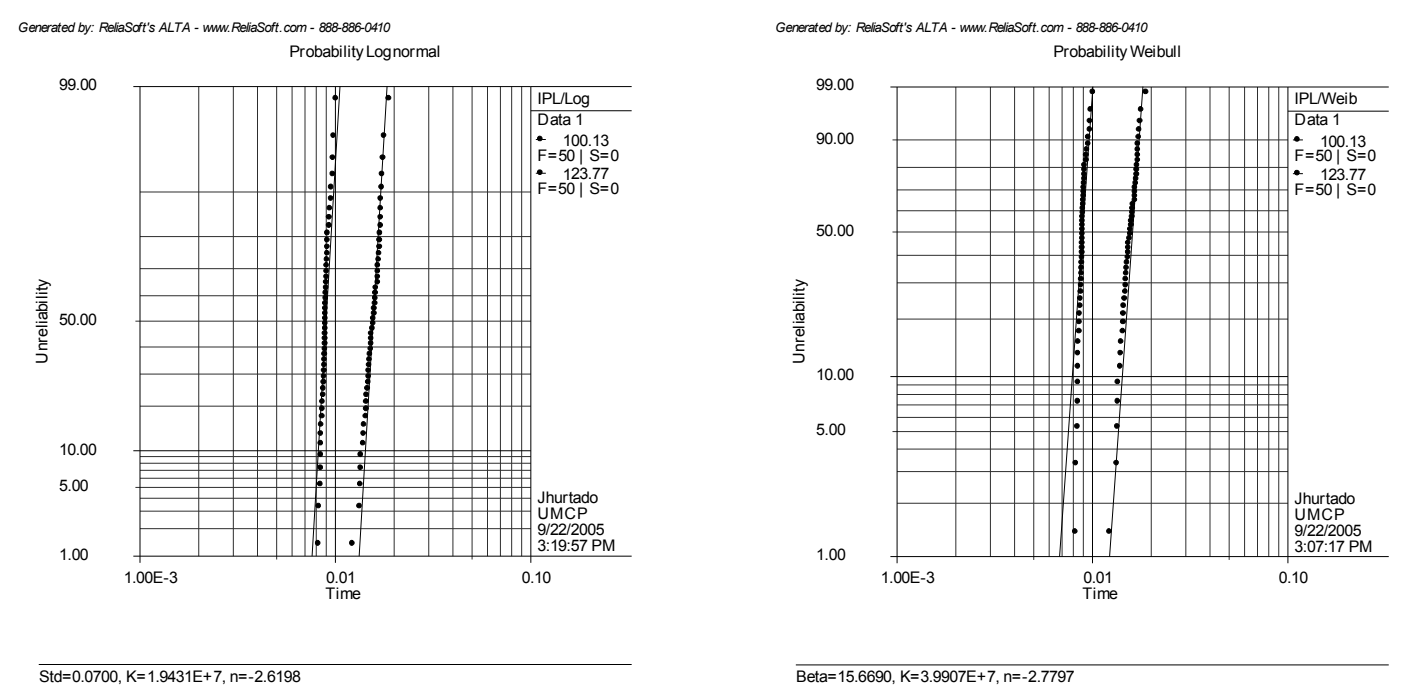

Figure 5.5 Lognormal and Weibull graphical comparison.

\subsection{Inverse Power Law Relationship (IPL) as a Representation of Life (life expended) and Crack Size.}

According to the physical relations discussed in Chapter 4, the IPL model is the appropriate form that can be used to represent crack growth as a function of time, cycle, or life-expended [90]. An IPL form showing two scalar quantities $x$ and $y$ can be written as

$$
y=a x^{b}
$$

where $a$ is the constant of proportionality and $b$ is the exponent of the power law. It can be displayed as a straight line on a log-log (natural logarithm is used in this study) relationship as,

$$
\operatorname{Ln}(y)=b \operatorname{Ln}(x)+\operatorname{Ln}(a)
$$


The special form of the IPL given by [91] is used in this research

$$
L(V)=\frac{1}{k V^{n}}
$$

where, $L$ is a quantifiable index of life measure (in our case crack size). May be hours as a function of "damage" index (in our case FLE) $V$. In (5.3) $k$ and $n$ are constant parameters of the model to be estimated.

Where fatigue crack occurs, the crack growth rate $(\mathrm{d} a / \mathrm{d} N)$ has a IPL relationship such as Equation 2.7.

Now that life distribution and damage-life (crack size vs. life expended) relationship have been defined, the relationship between these two will be discussed.

\subsection{IPL -Lognormal Relationship.}

The model for crack growth-damage is defined by Equations 4.6, 4.18 and 4.21 in the following form [38]

$$
N_{i f}=\frac{a_{f}^{1-m / 2}-a_{i}^{1-m / 2}}{C(F \Delta S \sqrt{\pi})^{m}(1-m / 2)}
$$

where $N_{i f}$ is the number of load cycles causing crack to grow from an initial crack size

$a_{i}$ to final crack size $a_{f}$. According to Equation 5.4, $N \propto a^{1 / n}$. Using FLE to measure life expended, one can conclude that

$$
F L E \propto \frac{N}{N_{\mathrm{f}}} \propto \frac{a^{1 / n}}{N_{\mathrm{f}}} \text { or } F L E \propto a^{1 / n}
$$

where $N_{f}$ is the number of cycles that the strain-based life model estimate for a crack to initiate. 
To incorporate the IPL model (given by Equation 5.3) into the model for crack growth life, crack growth can be itself the representation of the characteristic life, and since $\frac{1}{V} \propto a^{1 / n}$ (as noted in Equation 5.5), one may conclude that $F L E \propto a^{1 / n} \propto \frac{1}{V}$, where the life expended index $V=(F L E)^{-1 .}$

In summary, if $a \propto \frac{1}{V^{n}}$, the IPL model for the crack growth can be defined as:

$$
a\left(F L E^{-1}\right)=\frac{1}{k(F L E)^{-n}}
$$

Moreover, the IPL model and lognormal relationship are used to calculate the joint prior distribution of crack size $(a)$ and damage index $(V)$ as

$$
f(a, F L E)=\frac{1}{a \cdot \sigma_{a} \sqrt{2 \pi}} \exp \left[-\frac{1}{2}\left(\frac{a^{\prime}-\bar{a}^{\prime}}{\sigma_{a}}\right)^{2}\right]
$$

where,

$$
\begin{gathered}
a^{\prime}=\operatorname{Ln}(a) \\
\bar{a}^{\prime}=-\operatorname{Ln}(k)-n \operatorname{Ln}(V)=-\operatorname{Ln}(k)+n \operatorname{Ln}(F L E)
\end{gathered}
$$

Accordingly, the joint prior lognormal model $p d f$ for crack size " $a$ " and life expended index FLE is

$$
f(a, F L E)=\frac{1}{a \cdot \sigma_{a} \sqrt{2 \pi}} \exp \left[-\frac{1}{2}\left(\frac{a^{\prime}+\operatorname{Ln}(k)-n \operatorname{Ln}(F L E)}{\sigma_{a}}\right)^{2}\right]
$$

The parameters $k, n$, and $\sigma_{a}$ may be estimated using one of several possible methods. The data needed as inputs are the crack size, and the corresponding FLE values. The parameters for the prior $p d f$ are calculated using Equation 5.9. It should 
be noted that for an aircraft with a given value of $F L E=j \%$, the prior crack size distribution can be obtained using the following equation:

$$
f\left(a \mid F L E_{j \%}\right)=\frac{1}{a \cdot \sigma_{a} \sqrt{2 \pi}} \exp \left[-\frac{1}{2}\left(\frac{a^{\prime}+\operatorname{Ln}(k)-n \operatorname{Ln}\left(F L E_{j \%}\right)}{\sigma_{a}}\right)^{2}\right]
$$

The joint distribution of the crack growth and the IPL model is shown in Figure 5.6, where a typical $p d f$ of crack size is plotted and damage (FLE) is shown.

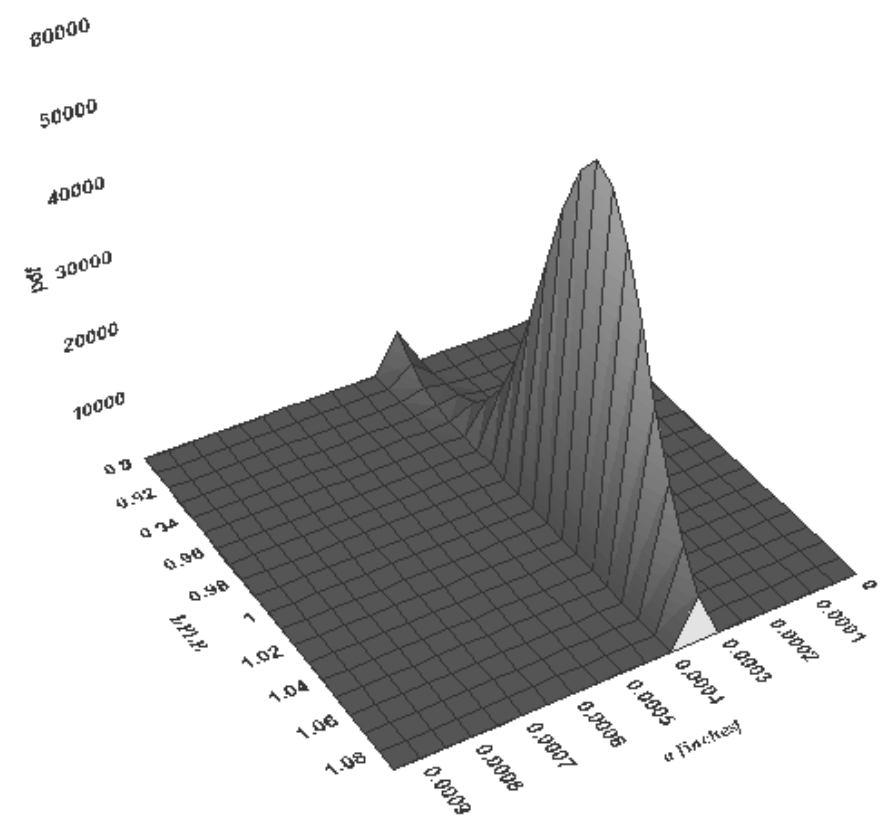

Figure 5.6 A three dimensional representation of the $p d f$ vs. crack size and FLE.

The next step is to choose one of the several methods to estimate the parameters of the $p d f$ model described by (5.10). Among these classical methods, we have the probability plotting, the least square, and the maximum likelihood estimation (MLE). In this work, the MLE is the method chosen. In addition, two relatively new techniques are also used. These methodologies are the Genetics Algorithm (GA) 
approach and Markov Chain Monte Carlo (MCMC), which is based on the subjectivist Bayesian estimation notion.

\subsection{Parameter Estimation Using MLE}

The most common inference technique used to estimate the "true value" of a parameter is the MLE, which uses values that maximize the likelihood $(L)$ of a sample [92]. The likelihood of a sample of $n$ observations $x_{1}, x_{2}, \ldots, x_{n}$, is the joint probability function $p\left(x_{1}, x_{2}, \ldots, x_{n}\right)$ where $x_{1}, x_{2}, \ldots, x_{n}$ are discrete random variables. If $x_{1}, x_{2}, \ldots, x_{n}$ are continuous random variables, then the likelihood of a sample of $n$ observations, $x_{1}, x_{2}, \ldots, x_{n}$, is the joint density function $f\left(x_{1}, x_{2}, \ldots, x_{n}\right)$. If $L$ is a

function of the parameters $\theta_{1}, \theta_{2}, \ldots \theta_{\mathrm{k}}$, the maximum likelihood estimators are the values of $\theta_{1}, \theta_{2}, \ldots \theta_{\mathrm{k}}$ that maximize $L$.

The IPL-lognormal log-likelihood function of Equation 5.10 is given by,

$$
\ln (L)=\Lambda=\sum_{i=1}^{F_{e}} N_{i} \ln \left[\frac{1}{\sigma_{a} T_{i}} \phi\left(\frac{\ln \left(a_{i}\right)+\ln (k)-n \ln \left(F L E_{i}\right)}{\sigma_{a}}\right)\right]
$$

where:

- $F_{e}$ is the number of groups of exact crack growth rate data points.

- $N_{i}$ is the number of crack growth rate data points in the $i^{\text {th }}$ crack growth rate data group.

- $\sigma_{a}$ is the standard deviation of the natural logarithm of the crack growth rate (unknown, the first of three parameters to be estimated). 
- $k$ is the IPL parameter (unknown, the second of three parameters to be estimated).

- $n$ is the second IPL power parameter (unknown, the third of three parameters to be estimated).

- $F L E_{i}$ is the damage level of the $i^{\text {th }}$ group.

- $a_{i}$ is the exact crack growth rate of the group.

The solution for $\hat{\sigma}_{a^{\prime}}, \hat{K}, \hat{n}$ will be found by solving

$\frac{\partial \Lambda}{\partial \hat{\sigma}_{a}}=0, \frac{\partial \Lambda}{\partial \hat{K}}=0$, and $\frac{\partial \Lambda}{\partial \hat{n}}=0$,

where

$$
\begin{aligned}
& \frac{\partial \Lambda}{\partial K}=-\frac{1}{K \sigma_{a}^{2}} \sum_{i=1}^{F e} N_{i}\left(\ln \left(a_{i}\right)+\ln (K)-n \ln \left(F L E_{i}\right)\right) \\
& \frac{\partial \Lambda}{\partial n}=-\frac{1}{\sigma_{a}^{2}} \sum_{i=1}^{F e} N_{i} \ln \left(F L E_{i}^{-1}\right)\left[\ln \left(a_{i}\right)+\ln (K)-n \ln \left(F L E_{i}\right)\right] \\
& \frac{\partial \Lambda}{\partial \sigma_{a}}=\sum_{i=1}^{F e} N_{i}\left[\frac{\left(\ln \left(a_{i}\right)+\ln (K)-n \ln \left(F L E_{i}\right)\right)^{2}}{\sigma_{a}^{3}}-\frac{1}{\sigma_{a}^{2}}\right]
\end{aligned}
$$

and,

$$
\begin{aligned}
& \phi(x)=\frac{1}{\sqrt{2 \pi}} e^{-\frac{1}{2}(x)^{2}} \\
& \Phi(x)=\frac{1}{\sqrt{2 \pi}} \int_{-\infty}^{x} e^{-\frac{1}{2}(t)^{2}} d t
\end{aligned}
$$

The MLE method has many advantages over other methods when dealing with large samples. Some of those advantages are [ 94]:

- It is asymptotically consistent, which means that as the sample size gets larger, the estimates converge to the right values. 
- It is asymptotically efficient, which means that for large samples, it produces the most precise estimates.

- It is asymptotically unbiased, which means that for large samples one expects to get the right value on average. The distribution of the estimates themselves is normal, if the sample is large enough, and this is the basis for the usual Fisher Matrix confidence bounds.

\subsection{Estimation of the Prior Distribution of Crack Size as Crack Grows for Bayesian Parameter Estimation}

The MLE estimation of the parameters of the model described in equation 5.10 for each model are shown in Tables $5.2-5.4$ at the FLE of interest. These can be considered as the prior distribution for additional Bayesian analysis discussed later.

Table 5.2 IPL-Lognormal mean parameters at the FLE showed - Based on Walker crack growth model.

\begin{tabular}{|c|c|c|c|c|c|c|}
\hline Aircraft & Location & $F L E_{j \%}$ & $k$ & $n$ & $\sigma_{a}$ & $\bar{a}_{\mathrm{p}}{ }^{\prime}$ \\
\hline 1 & 351 & 262.070 & $3.781 \mathrm{E}+09$ & 3.766 & 0.918 & -1.083 \\
\hline 2 & 351 & 249.969 & $5.157 \mathrm{E}+12$ & 5.275 & 0.799 & -0.146 \\
\hline 3 & 351 & 266.774 & $3.192 \mathrm{E}+12$ & 5.195 & 0.903 & 0.228 \\
\hline 4 & 351 & 167.107 & $4.594 \mathrm{E}+16$ & 7.205 & 0.754 & -1.487 \\
\hline 5 & 351 & 261.734 & $1.892 \mathrm{E}+12$ & 5.084 & 0.913 & 0.035 \\
\hline 6 & 351 & 286.539 & $8.164 \mathrm{E}+10$ & 4.428 & 0.970 & -0.074 \\
\hline 7 & 351 & 184.818 & $1.646 \mathrm{E}+13$ & 5.574 & 0.907 & -1.339 \\
\hline 8 & 351 & 227.468 & $1.448 \mathrm{E}+13$ & 5.540 & 0.900 & -0.238 \\
\hline 9 & 351 & 282.209 & $1.016 \mathrm{E}+12$ & 4.952 & 0.887 & 0.296 \\
\hline 10 & 351 & 231.367 & $2.608 \mathrm{E}+13$ & 5.691 & 0.940 & 0.092 \\
\hline 11 & 351 & 260.811 & $7.284 \mathrm{E}+10$ & 4.442 & 0.947 & -0.300 \\
\hline 12 & 351 & 259.977 & $3.021 \mathrm{E}+11$ & 4.701 & 0.917 & -0.295 \\
\hline 13 & 351 & 261.406 & $4.371 \mathrm{E}+11$ & 4.820 & 0.912 & 0.027 \\
\hline 14 & 351 & 257.347 & $3.044 \mathrm{E}+12$ & 5.212 & 0.943 & 0.184 \\
\hline
\end{tabular}




\begin{tabular}{|c|c|c|c|c|c|c|}
\hline 15 & 351 & 215.797 & $7.187 \mathrm{E}+12$ & 5.404 & 0.929 & -0.563 \\
\hline 16 & 351 & 204.943 & $4.033 \mathrm{E}+14$ & 6.300 & 0.929 & -0.098 \\
\hline 17 & 351 & 184.989 & $6.611 \mathrm{E}+17$ & 7.883 & 0.885 & 0.118 \\
\hline 18 & 351 & 178.613 & $7.562 \mathrm{E}+12$ & 5.404 & 0.997 & -1.633 \\
\hline 19 & 351 & 186.131 & $1.354 \mathrm{E}+08$ & 3.040 & 0.723 & -2.834 \\
\hline 20 & 351 & 204.518 & $7.874 \mathrm{E}+12$ & 5.396 & 0.939 & -0.985 \\
\hline 21 & 351 & 208.503 & $2.116 \mathrm{E}+12$ & 5.087 & 0.891 & -1.215 \\
\hline 22 & 351 & 210.690 & $1.145 \mathrm{E}+11$ & 4.447 & 0.999 & -1.670 \\
\hline 23 & 351 & 217.473 & $7.853 \mathrm{E}+09$ & 3.861 & 0.911 & -2.005 \\
\hline 24 & 351 & 153.734 & $7.649 \mathrm{E}+06$ & 2.400 & 0.280 & -3.764 \\
\hline 25 & 351 & 166.920 & $3.037 \mathrm{E}+09$ & 3.737 & 0.552 & -2.712 \\
\hline
\end{tabular}

Table 5.3. IPL-Lognormal mean parameters at the FLE showed - Based on Forman crack growth model.

\begin{tabular}{|c|c|c|c|c|c|c|}
\hline Aircraft & Location & $F L E_{j \%}$ & $K$ & $n$ & $\sigma_{a}$ & $\bar{a}_{\mathrm{p}}{ }^{\prime}$ \\
\hline 1 & 351 & 262.070 & $7.401 \mathrm{E}+10$ & 4.384 & 0.838 & -0.615 \\
\hline 2 & 351 & 249.969 & $8.504 \mathrm{E}+13$ & 5.869 & 0.785 & 0.329 \\
\hline 3 & 351 & 266.774 & $3.960 \mathrm{E}+14$ & 6.200 & 0.762 & 1.021 \\
\hline 4 & 351 & 167.107 & $1.915 \mathrm{E}+18$ & 7.999 & 0.747 & -1.154 \\
\hline 5 & 351 & 261.734 & $1.497 \mathrm{E}+14$ & 5.992 & 0.804 & 0.720 \\
\hline 6 & 351 & 286.539 & $1.326 \mathrm{E}+13$ & 5.498 & 0.784 & 0.894 \\
\hline 7 & 351 & 184.818 & $2.347 \mathrm{E}+15$ & 6.614 & 0.739 & -0.870 \\
\hline 8 & 351 & 227.468 & $4.734 \mathrm{E}+15$ & 6.749 & 0.745 & 0.531 \\
\hline 9 & 351 & 282.209 & $5.372 \mathrm{E}+13$ & 5.789 & 0.769 & 1.051 \\
\hline 10 & 351 & 231.367 & $2.269 \mathrm{E}+16$ & 7.107 & 0.721 & 1.029 \\
\hline 11 & 351 & 260.811 & $2.230 \mathrm{E}+13$ & 5.634 & 0.773 & 0.611 \\
\hline 12 & 351 & 259.977 & $2.011 \mathrm{E}+13$ & 5.561 & 0.794 & 0.289 \\
\hline 13 & 351 & 261.406 & $2.840 \mathrm{E}+13$ & 5.693 & 0.700 & 0.711 \\
\hline 14 & 351 & 257.347 & $1.093 \mathrm{E}+14$ & 5.968 & 0.757 & 0.797 \\
\hline 15 & 351 & 215.797 & $2.629 \mathrm{E}+15$ & 6.637 & 0.809 & 0.166 \\
\hline 16 & 351 & 204.943 & $3.945 \mathrm{E}+17$ & 7.755 & 0.806 & 0.760 \\
\hline 17 & 351 & 184.989 & $1.454 \mathrm{E}+21$ & 9.501 & 0.754 & 0.872 \\
\hline 18 & 351 & 178.613 & $1.651 \mathrm{E}+15$ & 6.527 & 0.915 & -1.197 \\
\hline 19 & 351 & 186.131 & $3.758 \mathrm{E}+08$ & 3.262 & 0.604 & -2.699 \\
\hline 20 & 351 & 204.518 & $5.168 \mathrm{E}+14$ & 6.282 & 0.837 & -0.453 \\
\hline 21 & 351 & 208.503 & $9.709 \mathrm{E}+13$ & 5.889 & 0.838 & -0.758 \\
\hline 22 & 351 & 210.690 & $1.128 \mathrm{E}+12$ & 4.953 & 0.902 & -1.250 \\
\hline 23 & 351 & 217.473 & $2.189 \mathrm{E}+11$ & 4.538 & 0.807 & -1.686 \\
\hline 24 & 351 & 153.734 & $1.916 \mathrm{E}+07$ & 2.600 & 0.174 & -3.676 \\
\hline 25 & 351 & 166.920 & $3.349 \mathrm{E}+09$ & 3.744 & 0.270 & -2.771 \\
\hline & & & & & & \\
\hline
\end{tabular}


Table 5.4 IPL-Lognormal mean parameters at the FLE showed - Based on Closure crack growth model.

\begin{tabular}{|c|c|c|c|c|c|c|}
\hline Aircraft & Location & $F L E_{j \%}$ & $K$ & $n$ & $\sigma_{a}$ & $\bar{a}_{\mathrm{p}}{ }^{\prime}$ \\
\hline 1 & 351 & 262.070 & $2.422 \mathrm{E}+05$ & 1.658 & 0.216 & -3.165 \\
\hline 2 & 351 & 249.969 & $1.305 \mathrm{E}+11$ & 4.391 & 0.795 & -1.352 \\
\hline 3 & 351 & 266.774 & $1.548 \mathrm{E}+11$ & 4.440 & 0.766 & -0.962 \\
\hline 4 & 351 & 167.107 & $4.355 \mathrm{E}+08$ & 3.246 & 0.196 & -3.279 \\
\hline 5 & 351 & 261.734 & $1.460 \mathrm{E}+10$ & 3.937 & 0.782 & -1.483 \\
\hline 6 & 351 & 286.539 & $8.448 \mathrm{E}+09$ & 3.818 & 0.783 & -1.256 \\
\hline 7 & 351 & 184.818 & $2.276 \mathrm{E}+07$ & 2.636 & 0.194 & -3.184 \\
\hline 8 & 351 & 227.468 & $1.826 \mathrm{E}+11$ & 4.492 & 0.718 & -1.553 \\
\hline 9 & 351 & 282.209 & $1.160 \mathrm{E}+10$ & 3.891 & 0.733 & -1.217 \\
\hline 10 & 351 & 231.367 & $2.544 \mathrm{E}+11$ & 4.581 & 0.729 & -1.323 \\
\hline 11 & 351 & 260.811 & $1.269 \mathrm{E}+09$ & 3.453 & 0.735 & -1.752 \\
\hline 12 & 351 & 259.977 & $9.135 \mathrm{E}+09$ & 3.841 & 0.786 & -1.577 \\
\hline 13 & 351 & 261.406 & $7.683 \mathrm{E}+09$ & 3.826 & 0.787 & -1.469 \\
\hline 14 & 351 & 257.347 & $3.202 \mathrm{E}+10$ & 4.124 & 0.743 & -1.301 \\
\hline 15 & 351 & 215.797 & $8.439 \mathrm{E}+10$ & 4.340 & 0.838 & -1.835 \\
\hline 16 & 351 & 204.943 & $5.132 \mathrm{E}+11$ & 4.757 & 0.687 & -1.643 \\
\hline 17 & 351 & 184.989 & $1.235 \mathrm{E}+15$ & 6.415 & 0.664 & -1.264 \\
\hline 18 & 351 & 178.613 & $1.978 \mathrm{E}+07$ & 2.603 & 0.221 & -3.305 \\
\hline 19 & 351 & 186.131 & $1.724 \mathrm{E}+04$ & 1.102 & 0.094 & -3.995 \\
\hline 20 & 351 & 204.518 & $4.099 \mathrm{E}+10$ & 4.171 & 0.723 & -2.243 \\
\hline 21 & 351 & 208.503 & $9.723 \mathrm{E}+06$ & 2.430 & 0.234 & -3.113 \\
\hline 22 & 351 & 210.690 & $7.366 \mathrm{E}+05$ & 1.883 & 0.180 & -3.434 \\
\hline 23 & 351 & 217.473 & $7.311 \mathrm{E}+04$ & 1.397 & 0.127 & -3.684 \\
\hline 24 & 351 & 153.734 & $9.987 \mathrm{E}+03$ & 0.970 & 0.054 & -4.323 \\
\hline 25 & 351 & 166.920 & $6.769 \mathrm{E}+04$ & 1.404 & 0.086 & -3.940 \\
\hline
\end{tabular}




\subsection{Markov Chain Monte Carlo (MCMC)}

The Markov Chain Monte Carlo simulations started in earnest with the 1953 publication of an article by Nicolas Metropolis et al. [95]. Since then simulations have become an indispensable tool in many branches of science. They allow integration over the posterior distribution of model parameters to make inferences or predictions about model parameters. The MCMC simulations are based on the Metropolis algorithm which makes use of the Gibb sampler [96].

Before introducing the Metropolis algorithm and the Gibbs sampler, a few introductory comments on the Monte Carlo integration must be made, because the Monte Carlo integration is used to approximate posterior (or marginal posterior) distributions required by a Bayesian analysis.

\subsubsection{Monte Carlo integration}

The original Monte Carlo approach was a method developed by the physicists to use random number generation to compute complex integrals [97]. This is done using the following,

$$
\int_{a}^{b} s(x) d x
$$

If $s(x)$ is defined as the product of a function $f(x)$ and a probability density function $p(x)$, over the interval $(a, b)$, then

$$
\int_{a}^{b} s(x) d x=\int_{a}^{b} f(x) p(x) d x=E_{p(x)}[f(x)]
$$


Hence that the integral can be expressed as an expectation of $f(x)$ over the density $p(x)$. Thus, if we draw a large number $x_{1}, \ldots, x_{n}$ of random variables from the density $p(x)$, then

$$
\int_{a}^{b} h(x) d x=E_{p(x)}[f(x)] \cong \frac{1}{n} \sum_{i=1}^{n} f\left(x_{i}\right)
$$

This is known as the Monte Carlo integration, and it can be used to approximate posterior distributions required for a Bayesian analysis. Consider the integral, $I(y)=\int f(y \mid x i) p(x) d x$, which we approximate by

$$
\hat{I}(y)=\frac{1}{n} \sum_{i=1}^{n} f\left(y \mid x_{i}\right)
$$

where $x_{i}$ are draws from the density $p(x)$.

The Monte Carlo integration draws samples from the required distribution and then forms sample averages to approximate expectations. MCMC draws these samples by running a Markov chain for a long time. Although the chains may be constructed using various methods, Gibbs sampling is one of the most popular methods (a special case of the Metropolis-Hastings method) [98].

\subsubsection{Metropolis-Hasting and Gibbs Sampling}

One problem with applying the Monte Carlo integration is in obtaining samples from complex $p d f p(x)$. Mathematical physicists attempted to solve this problem by integrating very complex functions by random sampling $[98,99,100]$. The result was the Metropolis-Hastings algorithm. A detailed review of this method is given by Chib and Greenberg [101]. 
The Metropolis-Hasting algorithm can draw samples from any probability distribution $p(x)$, requiring only that the density can be calculated at $x$. The algorithm generates a Markov chain in which each state $x^{t}$ depends only on the previous state $x^{t-}$

1. The algorithm uses a proposal density $q\left(x^{*}, x^{t}\right)$, which depends on the current state $x^{t}$, to generate a new proposed sample $x^{*}$. By using the following procedure, the algorithm generates a sequence of draws from this distribution:

1. At $t=1$, fix $x^{0}$.

2. Simulate state $x^{*}$ from density $\mathrm{x}^{*} \sim q\left(x^{t-1}, \cdot\right)$.

3. Given the candidate point $x^{*}$, calculate the ratio of the density at the candidate $\left(x^{*}\right)$ and current $\left(x^{t-1}\right)$ points,

$$
\alpha=\min \left(\frac{p\left(x^{*}\right)}{p\left(x^{t-1}\right)} \frac{q\left(x^{*}, x^{t-1}\right)}{q\left(x^{t-1}, x^{*}\right)}, 1\right)
$$

4. Decide acceptance.

If $u \leq \alpha\left(x^{t-1}, x^{*}\right)$, then accept transition: $x^{t}=x^{*}$

else $u>\alpha\left(x^{t-1}, x^{*}\right)$, then stay in the present state $x^{t}=x^{t-1}$.

5. $t=t+1$, go to step 2 .

This generates a Markov chain $\left(x^{0}, x^{1}, \ldots, x^{q}, \ldots\right)$, as the transition probabilities from $x^{t}$ to $x^{t+1}$ depends only on $x^{t}$. Following a sufficient burn-in period (of, say, $q$ steps), the chain approaches its stationary distribution and, samples from the vector $\left(x_{q+1}, \ldots, x_{q+n}\right)$ are samples from $p(x)$.

This is the Metropolis-Hastings algorithm. By assuming that the proposal distribution is symmetric, i.e., $q(x, y)=q(y, x)$, the original Metropolis algorithm may be recovered. 
The Gibbs sampler is a special case of Metropolis-Hastings sampling where the acceptance probability is always 1(i.e. $\alpha=1$ ) [102]. The task remains to specify how to construct a Markov Chain whose values converge to the target distribution. The key to the Gibbs sampler is that one considers only univariate conditional distributions (the distribution when all of the random variables but one is assigned fixed values). Such conditional distributions are far easier to simulate than complex joint distributions and usually have simple forms (often being normal, inverse $\chi^{2}$, or other common prior distributions). Thus, one simulates $n b$ random variables sequentially from the $n b$ univariate conditionals rather than generating a single $n b$ dimensional vector in a single pass using the full joint distribution.

To introduce the Gibbs sampler, consider a bivariate random variable $(x, y)$, and suppose we wish to compute one or both marginals, $p(x)$ and $p(y)$. The idea behind the sampler is that it is far easier to consider a sequence of conditional distributions, $p(x \mid y)$ and $p(y \mid x)$, than it is to obtain the marginal by integration of the joint density $p(x, y)$, e.g., $p(x)=\int p(x, y) d y$. The sampler starts with an initial value $y_{0}$ for $y$ and obtains $x_{0}$ by generating a random variable from the conditional distribution $p\left(x \mid y=y_{0}\right)$. The sampler then uses $x_{0}$ to generate a new value of $y_{1}$, drawing from the conditional distribution based on the value $x_{0}, p\left(y \mid x=x_{0}\right)$. The sampler proceeds as follows

$$
x_{i} \sim p\left(x \mid y=y_{i-1}\right) \text { and } y_{i} \sim p\left(y \mid x=x_{i}\right)
$$

Repeating this process $r$ times, generates a Gibbs sequence of length $q$, where a subset of points $\left(x_{j}, y_{j}\right)$ for $1 \leq j \leq m<q$ are taken as our simulated draws from the full joint distribution. (One iteration of all the univariate distributions is often called a 
scan of the sampler. To obtain the desired total of $m$ sample points (here each "point" on the sampler is a vector of the two parameters), one samples the chain (i) after a sufficient burn-in to remove the effects of the initial sampling values and (ii) at set time points (say every $n b$ samples) following the burn-in. The Gibbs sequence converges to a stationary (equilibrium) distribution that is independent of the starting values. By construction this stationary distribution is the target distribution being simulated [103]

For evaluating the IPL-lognormal parameters, Equation 5.12 is programmed in the code WinBUGS [104], which is a free software package that implements Gibbs sampling under a wide variety of conditions.

\subsubsection{Burn-in period}

A key issue in the successful implementation of the MCMC sampler is the number of runs (steps) taken until the chain approaches stationarity (the length of the burn-in period). Typically the first 1000 to 5000 elements are thrown out. A poor choice of starting values and/or proposal distribution can greatly increase the required burn-in time, and an area of much current research is whether an optimal starting point and proposal distribution can be found. One suggestion for a starting value is to start the chain as close to the center of the distribution as possible, for example taking a value close to the distribution's mode (such as using an approximate MLE as the starting value) [97]. 


\subsection{Genetic Algorithm (GA)}

A revolution in biological thought, and indeed in human philosophy, began when Charles Darwin and Alfred Russel Wallace presented their evidence for the theory of evolution before the Linnean Society of London on July 1, 1858. Classical Darwinian evolutionary theory, combined with the selectionism of Weismann and the genetics of Mendel, has now become a rather universally accepted set of arguments known as the neo-Darwinian paradigm [105].

This paradigm asserts that the history of the vast majority of life is fully accounted for by only a very few statistical processes acting on and within populations and species. These processes are reproduction, mutation, competition, and selection. Reproduction is an obvious property of all life; mutation is guaranteed in any system that continuously reproduces itself in a positively entropic universe. Competition and selection become the inescapable consequences of any expanding population constrained to a finite arena. Evolution is then the result of these fundamental, interacting, stochastic processes as they act on populations, generation after generation [105].

The idea of using evolutionary computation as a problem solving technique has existed since the 1950s. Since then, four major approaches have evolved: Evolutionary Programming, Evolutionary Strategies, Genetic Algorithms and Genetic Programming. All of these are algorithms that have been inspired by the notions of evolution and survival in nature [106]. In this research, GA is used to estimate the parameters of the IPL-lognormal model. 
The GA was first introduced by Holland [107] to find approximate solutions to optimization problems. This methodology is designed to mimic the natural genetic behaviors by incorporating specific mathematical operators to replicate processes such as crossover, mutation, and recombination. The generic approach to GA is implemented as follows:

1. The problem to be addressed is defined as an objective function that indicates the fitness of any potential solution.

2. A population of candidate solutions (in our case parameters of the IPLlognormal model) is initialized. Typically, each trial is coded as a vector $\mathrm{x}$,(chromosome) with its elements (genes) and varying values at specific positions (alleles).

3. Each chromosome in the population is decoded into a form appropriate for evaluation and is then assigned a fitness score, according to the objective.

4. A probability of reproduction is assigned to each chromosome. The likelihood of being selected is proportional to its fitness relative to the other chromosomes in the population. Techniques such as the roulette wheel, tournament, and stochastic universal sampling are used to select the parents.

a. Roulette wheel: In order to reproduce offspring, parents need to be selected. The most commonly used methods are roulette wheel selection and rank selection. The key for roulette wheel selection is fitness. The fitter the chromosomes are, the more chances they will have to be selected. Imagine a roulette where each chromosome is placed in its own section 
according to its fitness function. The fittest one gets the largest area, while the least fit gets the smallest area [108].

b. Tournament: In tournament, the selection of individuals is randomly chosen from the population. They may be drawn from the population with or without replacement. These individuals take part in a tournament whereby the winning individual is determined depending on its fitness value. The individual having the highest fitness value is usually chosen deterministically although a stochastic selection may occasionally be made. In both cases only the winner is inserted into the next population and the process is repeated $\mathrm{n}$ times to obtain a new population [109].

c. Stochastic universal sampling (SUS): This is a simple, single-phase sampling algorithm. This method provides zero bias and minimum spread. The individuals are mapped to contiguous segments of a line, such that each individual's segment is equal in size to its fitness exactly as in roulette-wheel selection. Hence, SUS has minimal spread. Furthermore, in a randomly ordered population, an individual's selection probability is based solely on the initial spin and the magnitude of his expected value. Hence, SUS has zero bias [110].

5. A new population of chromosomes is generated according to the assigned probabilities of reproduction. Operators such as discrete recombination, intermediate recombination, extended line recombination and mutation are used to generate the new chromosome or offspring. These operators work in the following way: 
a. Discrete recombination: This operator performs an exchange of variable values between the individuals [111]. For each variable, the parent who contributes its variable to the offspring is chosen randomly with equal probability. Typical values for the probabilities of recombination are 0.6 [112], 0.75 to 0.95 [113] and 0.95 [114]. Discrete recombination can be used with any kind of variables (binary, real or symbols).

b. Intermediate recombination: Here the variable values of the offspring are chosen somewhere around and between the variable values of the parents [109]. Offspring are produced according to the rule:

Offspring $=$ parent $1+\kappa($ parent $2-$ parent 1$)$ where $\kappa$ is a scaling factor chosen uniformly at random over an interval [$d, 1+d]$. In intermediate recombination $d=0$, for extended intermediate recombination $d>0$. A good choice is $d=0.25$. Each variable in the offspring is the result of combining the variables according to the above expression with a new $\alpha$ chosen for each variable. It is only applicable to real variables.

c. Extended line recombination: It generates offspring in a direction defined by the parents (line recombination). It tests more often outside the area defined by the parents and in the direction of parent 1 . The point for the offspring is defined by features of the mutation operator of the Breeder GA [113]. It is only applicable to real variables.

d. Mutation: This operator is applied after recombination, and plays an important role in genetic algorithm, because it helps to prevent the 
problems of premature convergence associated with the repeated use of crossover. Offspring variables are mutated by the addition of small random values (size of the mutation step) with low probability [116]. Typical values for the probabilities of mutation are 0.001 [110], 0.005 to 0.01 [113] and 0.01 [114]. Mutation is considered a background operator, ensuring that the crossover has a full range of alleles and that the adaptive plan is not trapped on local optima. Typically, a point is considered locally optima if no improvement can be made by searching in a nonempty neighborhood around that point. This may not be the case for genetic algorithms relying solely on crossover. The sequence of trials may stagnate at any homogeneous collection of points. Under such conditions, the point is locally optima only because the search algorithm is incapable of proceeding further [105].

6. The process stops if a suitable solution has been achieved or if the computer time has expired. Otherwise, the process proceeds to step three, where the new chromosomes are scored.

A numerical function calculating the parameters of the IPL-lognormal model was developed on MATLAB ${ }^{\circledR}$ and the Genetic and Evolutionary Algorithm Toolbox created for use with MATLAB $^{\circledR}$ [116]. Figure 5.7 shows the structure of the algorithm. 


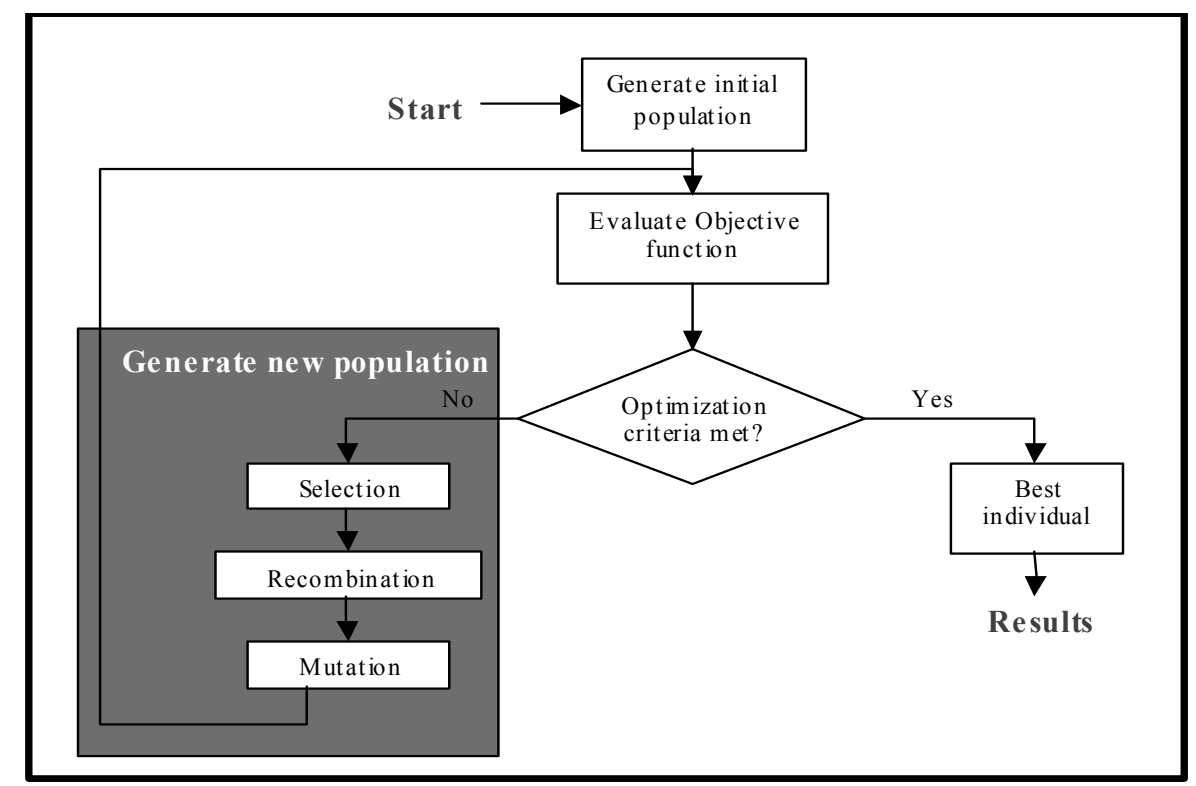

Figure 5.7 Genetic Algorithm structure.

The assumptions, values and functions used in the calculations are explained in the following paragraphs:

- Coding: A real variable coding is used in the algorithms. The use of binary strings is not universally accepted in the GA literature. Michalewicz [117] indicated that for real-valued numerical optimization problems, floating-point representations outperform binary representation, because they are more consistent, more precise, and more quickly executed.

- Generations and Population size: These two variables are tightly intertwined in the GA solutions. A big population size will increase the computational time for each generation. As part of this research, different 
population sizes and generation numbers were tested. A size population of 60 and generation number of 250 showed the best results.

- Parent Selection technique: In this research we used Roulette wheel, Tournament, and Stochastic Universal Sampling functions.

- Recombination operator: A probability of 0.6 was used in the routine for the operators: Discrete, Intermediate and Extended line recombination.

- Mutation operator: In this case, we use the mutation function for real values.

- Objective function: In this case, the objective function is the likelihood function of the IPL-Lognormal model.

The likelihood equation of the IPL-lognormal model is obtained through Equation 5.12, which is implemented in the GA routine to estimate the parameters $k$, $n$ and $\sigma_{a}$ such that $\Lambda$ becomes maximum in Equation 5.12. Ten data sets were analyzed using all parent selection techniques, and genetic operators to find the best combination that maximize the likelihood. From this analysis, it was observed that the three parent selection and the discrete recombination operator showed the best results. Figure 5.8 and Figure 5.9 illustrate how the likelihood equation converges to the optimum value in two examples. 


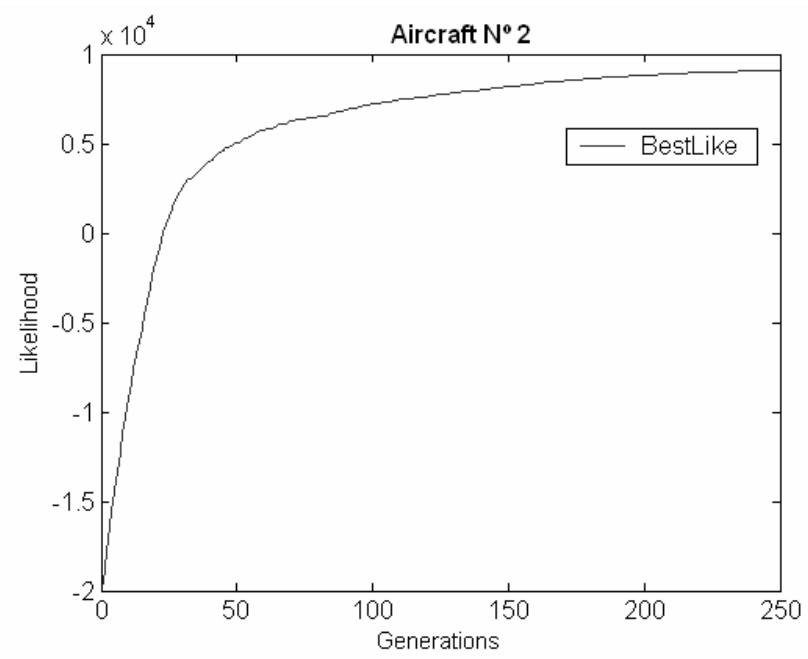

Figure 5.8 Likelihood and average likelihood for aircraft 2.

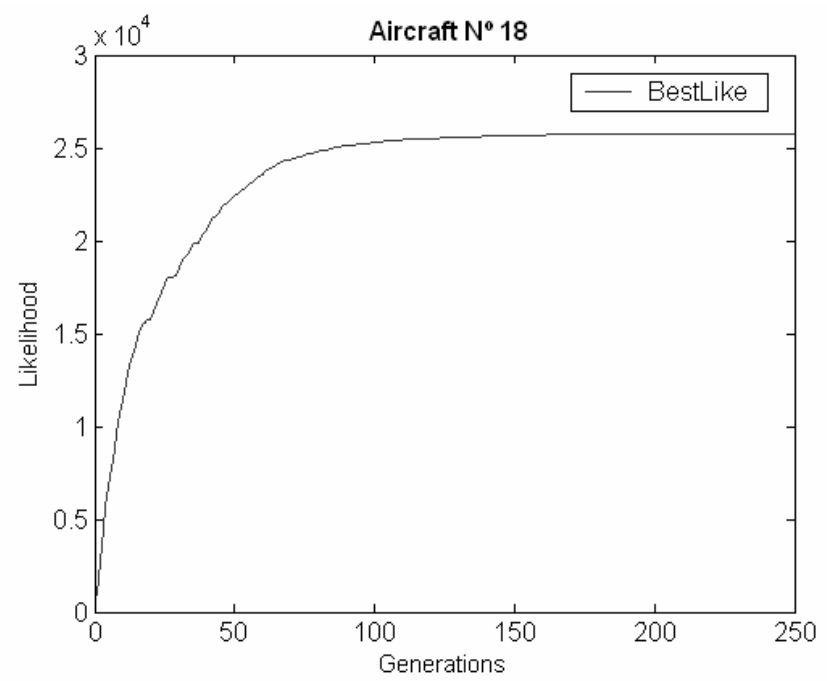

Figure 5.9 Likelihood and average likelihood for aircraft 18 .

Table 5.4 shows a comparison of the mean values of parameters estimated for 10 aircrafts using the three methodologies discussed. The MLE and MCMC methods produce very close estimates; however GA results are closed but somewhat different. Determining the best results depends on the characteristics of the data and the application in which the parameters will be used. 
As was mentioned previously, several methods may be used to estimate the model's parameters (MLE, MCMC, GA, etc). Of these, the MLE method is preferred because; it is very precise when dealing with large amount of data, which is the case in this research ( $>600$ data points). One of the weaknesses of the MCMC method is the starting values for the empirical distribution, and to solve this, we recommend that values from a previous MLE analysis be used. Although GA has proven to be an efficient and powerful problem-solving strategy, it also has certain limitations. If the population size is too small, the genetic algorithm may not explore enough of the solution space to consistently find good solutions. If the rate of genetic change is too high or the parent selection is chosen poorly, the results may be poor.

Table 5.5 IPL-Lognormal mean parameters values using three estimation methodologies.

\begin{tabular}{|l|c|c|c|c|c|c|c|c|c|}
\hline & \multicolumn{3}{|c}{ MLE } & \multicolumn{3}{c|}{ MCMC } & \multicolumn{3}{c|}{ GA } \\
\hline & $K$ & $n$ & $\sigma_{a}$ & $K$ & $n$ & $\sigma_{a}$ & $K$ & $n$ & $\sigma_{a}$ \\
\hline 1 & $2.422 \mathrm{E}+05$ & 1.658 & 0.216 & $2.374 \mathrm{E}+05$ & 1.654 & 0.216 & $3.001 \mathrm{E}+05$ & 1.704 & 0.341 \\
\hline 2 & $1.305 \mathrm{E}+11$ & 4.391 & 0.795 & $1.270 \mathrm{E}+11$ & 4.385 & 0.796 & $2.467 \mathrm{E}+11$ & 4.520 & 0.759 \\
\hline 3 & $1.548 \mathrm{E}+11$ & 4.440 & 0.766 & $1.574 \mathrm{E}+11$ & 4.444 & 0.767 & $3.285 \mathrm{E}+11$ & 4.583 & 0.795 \\
\hline 4 & $4.355 \mathrm{E}+08$ & 3.246 & 0.196 & $4.308 \mathrm{E}+08$ & 3.243 & 0.197 & $3.331 \mathrm{E}+08$ & 3.191 & 0.198 \\
\hline 7 & $2.276 \mathrm{E}+07$ & 2.636 & 0.194 & $2.162 \mathrm{E}+07$ & 2.625 & 0.194 & $2.501 \mathrm{E}+07$ & 2.654 & 0.228 \\
\hline 13 & $7.683 \mathrm{E}+09$ & 3.826 & 0.787 & $7.541 \mathrm{E}+09$ & 3.822 & 0.788 & $6.860 \mathrm{E}+09$ & 3.802 & 0.732 \\
\hline 14 & $3.202 \mathrm{E}+10$ & 4.124 & 0.743 & $3.319 \mathrm{E}+10$ & 4.131 & 0.744 & $2.801 \mathrm{E}+10$ & 4.097 & 0.740 \\
\hline 18 & $1.978 \mathrm{E}+07$ & 2.603 & 0.221 & $1.958 \mathrm{E}+07$ & 2.600 & 0.221 & $2.504 \mathrm{E}+07$ & 2.645 & 0.244 \\
\hline 20 & $4.099 \mathrm{E}+10$ & 4.171 & 0.723 & $4.244 \mathrm{E}+10$ & 4.178 & 0.723 & $4.088 \mathrm{E}+10$ & 4.171 & 0.727 \\
\hline 25 & $6.769 \mathrm{E}+04$ & 1.404 & 0.086 & $6.777 \mathrm{E}+04$ & 1.404 & 0.086 & $6.312 \mathrm{E}+04$ & 1.389 & 0.095 \\
\hline
\end{tabular}

Because of this, MLE is the preferred technique to estimate the model parameters (in the prior joint density function) in this research. The results are 
considered reasonable because of the large size of the sample and of the small amount of time and computational effort that it takes to do the calculation [118].

Accordingly, for a given aircraft with $F L E=j \%$, the prior $p d f$ of crack size can be determined by Equation 5.11. By combining the evidence of cracks for an aircraft at $F L E=j \%$ with this prior $p d f$, Bayesian updating mathematics can be used to generate the posterior distribution. This procedure is discussed in the Chapter 6 . 


\section{Chapter 6 Bayesian Updating Process of the Crack Size}

\section{Distribution}

Classical inferential models do not permit the introduction of prior knowledge into the calculations. For the rigor of the scientific method, this is an appropriate response to prevent the introduction of extraneous data that might skew the experimental results. However, there are times when the use of prior knowledge would be a useful contribution to the evaluation process [119].

Bayesian inference is proposed as a model for updating probabilities via Bayes' Theorem, in which one starts with an initial set of beliefs about the relative plausibility of various hypotheses, collects new information, and adjusts the original set of beliefs in light of the new information to produce a more refined set of beliefs.

Bayes' Theorem has proven to be a coherent method of mathematically expressing a decrease in uncertainty gained by an increase in knowledge [120]. Bayes' Theorem is mathematically expressed as:

$$
P\left(H_{0} \mid E\right)=\frac{P\left(H_{0}\right) \cdot\left(P\left(E \mid H_{0}\right)\right.}{P(E)}
$$

where $H_{0}$ represents a hypothesis, called a null hypothesis that was inferred before new evidence $E$, became available; $P\left(H_{0}\right)$ is called the prior probability of $H_{0} ; P\left(E \mid H_{0}\right)$ is called the conditional probability of seeing the evidence $E$ given that the hypothesis $H_{0}$ is true. It is also called the likelihood function; $P(E)$ is the probability of witnessing the new evidence $E$ under all mutually exclusive hypotheses. It is also called the marginal probability and can be calculated as the sum of the product of all probabilities of 
mutually exclusive hypotheses and corresponding conditional probabilities; $P\left(H_{0} \mid E\right)$ is called the posterior probability of $H_{0}$ given $E$.

It is important to note that the factor $P\left(E \mid H_{0}\right) / P(E)$ represents the impact that the evidence has on the belief in the hypothesis. Multiplying the prior probability of the hypothesis by this factor would result in a large posterior probability of the hypothesis given the evidence. Bayes' theorem therefore measures how much new evidence should alter a belief in a hypothesis [121].

In this research, the prior knowledge about the initial crack size at $100 \%$ FLE is represented by a probability density function in Chapter 3. If further inspection is carried out on the aircrafts, and new evidence of cracks obtained, the Bayes' theorem will allow us to calculate a new $p d f$ that reflects this update of knowledge.

\subsection{Bayesian Updating of Crack Size Distribution}

The Updating of the prior initial crack size $p d f$ information is carried out using crack evidence from the inspection of the aircrafts coupled with a proper prior distribution of such cracks. The updating process is based on the Bayesian approach, which provides a mechanism of updating one's degree of belief about the crack size in light of any new crack evidence data such as inspection results, according to,

$$
\pi(a \mid E)=\frac{1}{h} L(E \mid a) F(a)
$$

and

$$
h=L(E)=\int L(E \mid a) F(a) \mathrm{d} a
$$


where $\pi(a \mid E)=$ posterior updated distribution of crack size, $a$, given evidence $E, L(E \mid a)=$ likelihood function or probability of observing evidence $E$ given $a$, and $F(a)=$ prior probability distribution of $a$.

The calculations are performed assuming non-conjugate lognormal prior distribution of crack size at $F L E=j \%$, which is defined by [122]

$$
F\left(a \mid \lambda_{0}, \delta_{0}\right)_{F L E=J \%}=\frac{1}{a \delta_{0} \sqrt{2 \pi}} \exp \left[-\frac{1}{2}\left(\frac{\operatorname{Ln}(a)-\operatorname{Ln}\left(\lambda_{0}\right)}{\delta_{0}}\right)^{2}\right]
$$

where $\lambda_{0}$ and $\delta_{0}$ are the parameters of the prior distribution

Assume a lognormal likelihood function $(L)$ :

$$
L\left(a_{*} \mid a\right)_{F L E=J \%}=\frac{1}{a \delta_{*} \sqrt{2 \pi}} \exp \left[-\frac{1}{2}\left(\frac{\operatorname{Ln}(a)-\operatorname{Ln}\left(\lambda_{*}\right)}{\delta_{*}}\right)^{2}\right]
$$

$\lambda_{*}$, and $\delta_{*}$ are parameters of the likelihood distribution and, $a_{*}$ is the distribution of evidence (i.e. crack sizes observed).

Using the Bayes Theorem, posterior distribution is:

$$
\pi\left(a \mid a_{*}\right)_{F L E=J \%}=\frac{L\left(a_{*} \mid \lambda_{*} \delta_{*}\right) F\left(a \mid \lambda_{0} \delta_{0}\right)}{L\left(a_{*}\right)}
$$

where

$$
L\left(a_{*}\right)=\int_{0}^{\infty} L\left(a_{*} \mid a\right) F\left(a \mid \lambda_{0} \delta_{0}\right) \mathrm{d} a
$$

Posterior distribution is also lognormal:

$$
\pi\left(a \mid \lambda^{\prime}, \delta^{\prime}\right)_{F L E=j^{\circ} \%}=\frac{1}{a \delta^{\prime} \sqrt{2 \pi}} \exp \left[-\frac{1}{2}\left(\frac{\operatorname{Ln}(a)-\operatorname{Ln}\left(\lambda^{\prime}\right)}{\delta^{\prime}}\right)^{2}\right]
$$

In this particular case (i.e. lognormal prior and likelihood), $\lambda^{\prime}$ and $\delta^{\prime}$, parameters of the posterior distribution function are calculated from [123]:

$$
\lambda^{\prime}=\lambda_{0}^{w_{0}} \cdot \lambda_{*}^{w_{1}} \quad \text { and } \delta^{\prime}=\frac{1}{\sqrt{\frac{1}{\delta_{0}^{2}}+\frac{1}{\delta_{*}^{2}}}}
$$


where

$$
w_{0}=\frac{\delta_{*}^{2}}{\delta_{0}^{2}+\delta_{*}^{2}} \quad w_{1}=\frac{\delta_{0}^{2}}{\delta_{0}^{2}+\delta_{*}^{2}} \text { and } w_{0}+w_{1}=1
$$

Accordingly, for a given aircraft with $F L E=j \%$, the prior $p d f$ of crack size can be described by Equation 5.11. Through Bayesian mathematics, the prior $p d f$ is updated with the evidence of cracks for an aircraft at $F L E=j \%$, resulting in the posterior distribution for crack sizes at $F L E=j \%$. Consequently, the same updated parameters in Equation 5.11 are applicable for updated parameters to Equation 5.10. Figure 6.1 illustrates this process.

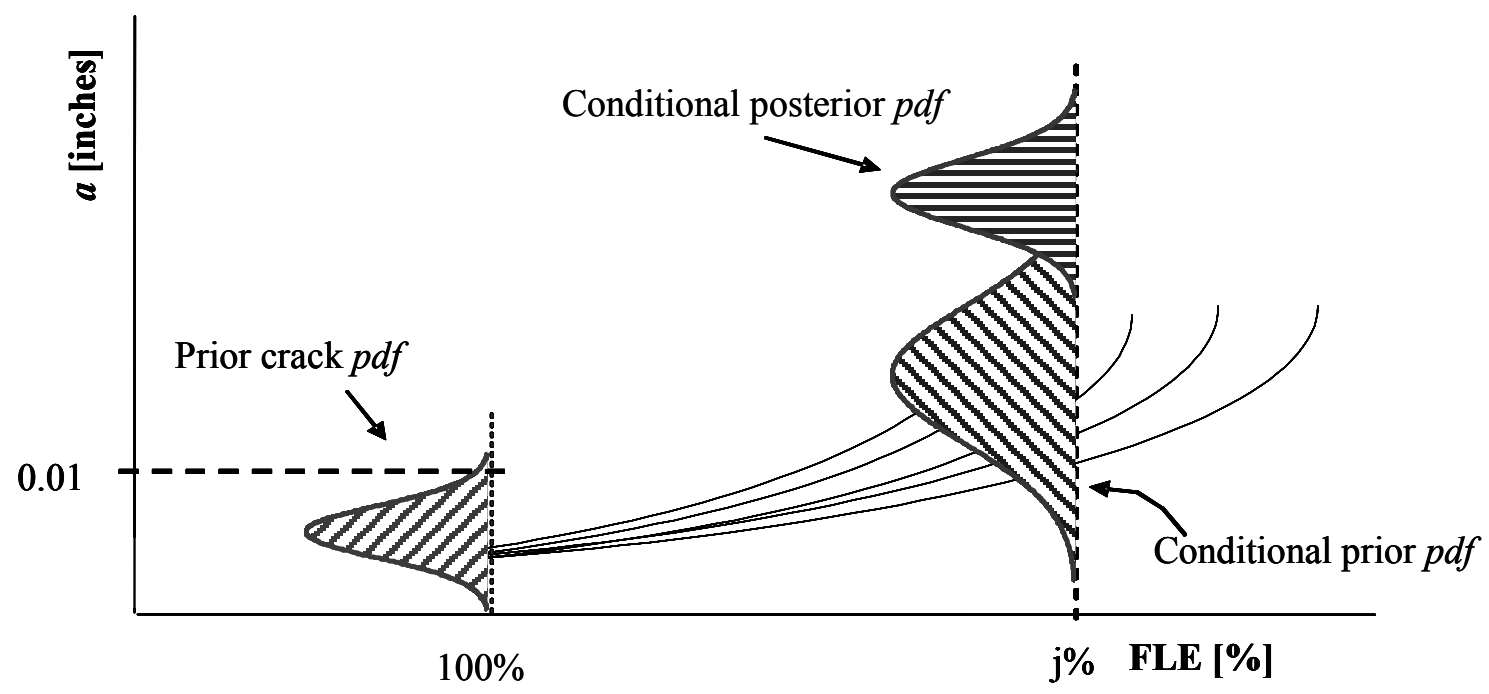

Figure 6.1 Updating process at the FLE of interest.

The parameters of the prior distribution are calculated from Equation 5.13 and are tabulated in Tables 5.1 - 5.3. The likelihood distribution function is calculated using the methodology developed by Groen [123], which is based on the sequential conjugate of the lognormal. This methodology uses the evidence, assuming that each value is an observation to be used in characterizing the variability in the parameter of interest. Such 
variability is represented by a range of values around the observations (evidence) made. This range, which is considered the uncertainty of the inspection findings, is calculated through an Error Factor (EF). To deal with this type of data, the methodology has two probability distribution options: normal, for evidence in the form of an observation and additive error factor, and lognormal, for evidence in the form of an observation and multiplicative error factor.

In this research, a lognormal $p d f$ is used, according to,

$$
\begin{aligned}
& \text { Upper bound }=\text { median } \cdot \mathrm{EF} \text {. } \\
& \text { Lower bound }=\text { median } / \mathrm{EF} \text {. }
\end{aligned}
$$

As the crack sizes are defined in the inspection data report (evidence) as: $\mathrm{XS}=$ $0.04 " ; \mathrm{S}=0.09 ", \mathrm{~L}=0.17$ "; $\mathrm{XL}=0.35$ ", then assuming these are mean values, the following $\mathrm{EF}$ 's are assigned to cover the possible ranges of crack size $\mathrm{EF}_{\mathrm{XS}}=1.5 ; \mathrm{EF}_{\mathrm{S}}=$ 1.5, $\mathrm{EF}_{\mathrm{L}}=1.25 ; \mathrm{EF}_{\mathrm{XL}}=1.6$. Figure 6.2 shows the ranges assigned to each crack size bin category.

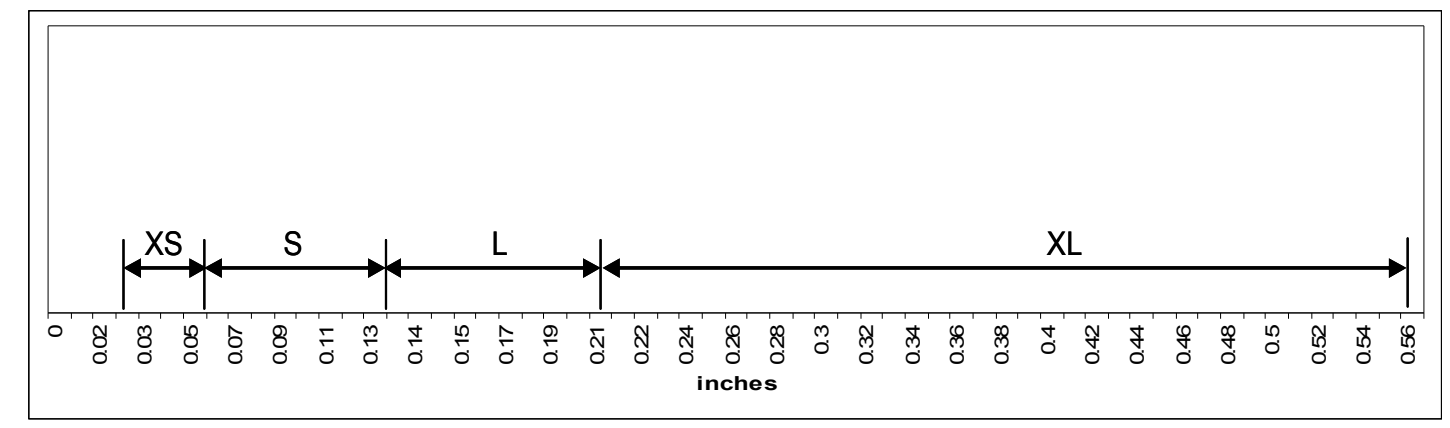

Figure 6.2 Crack size ranges.

Once the evidence is introduced, in combination with the prior $p d f$ model calculated in Chapter 5, the posterior (updated) distribution of crack size is obtained 
through the conjugate of the lognormal distribution. Table 6.1 shows the updated values for the three models, and Figure 6.3 displays the results.

Table 6.1 Updated Lognormal Parameters for the Three Crack Growth models -

Conjugate.

\begin{tabular}{|r|c|c|c|c|c|c|c|c|c|}
\cline { 2 - 11 } \multicolumn{1}{c|}{} & \multicolumn{3}{|c|}{ Walker } & \multicolumn{3}{c|}{ Forman } & \multicolumn{3}{c|}{ Closure } \\
\cline { 2 - 11 } \multicolumn{1}{c|}{} & $\mu_{\text {up }}$ & $\sigma_{\text {up }}$ & $\mu_{\text {upLn }}$ & $\mu_{\text {up }}$ & $\sigma_{\text {up }}$ & $\mu_{\text {upLn }}$ & $\mu_{\text {up }}$ & $\sigma_{\text {up }}$ & $\mu_{\text {upLn }}$ \\
\hline 1 & 0.064 & 0.171 & -2.754 & 0.066 & 0.171 & -2.723 & 0.052 & 0.136 & -2.953 \\
\hline 2 & 0.042 & 0.087 & -3.182 & 0.042 & 0.087 & -3.175 & 0.041 & 0.087 & -3.197 \\
\hline 3 & 0.045 & 0.171 & -3.094 & 0.049 & 0.170 & -3.008 & 0.045 & 0.170 & -3.108 \\
\hline 4 & 0.091 & 0.093 & -2.394 & 0.092 & 0.093 & -2.389 & 0.077 & 0.084 & -2.568 \\
\hline 5 & 0.043 & 0.141 & -3.142 & 0.045 & 0.140 & -3.099 & 0.042 & 0.140 & -3.163 \\
\hline 6 & 0.104 & 0.239 & -2.266 & 0.121 & 0.235 & -2.111 & 0.100 & 0.235 & -2.305 \\
\hline 7 & 0.041 & 0.122 & -3.184 & 0.043 & 0.122 & -3.156 & 0.040 & 0.104 & -3.209 \\
\hline 8 & 0.164 & 0.107 & -1.806 & 0.169 & 0.107 & -1.780 & 0.162 & 0.107 & -1.823 \\
\hline 9 & 0.052 & 0.238 & -2.966 & 0.060 & 0.235 & -2.822 & 0.049 & 0.234 & -3.018 \\
\hline 10 & 0.123 & 0.126 & -2.098 & 0.130 & 0.126 & -2.043 & 0.121 & 0.126 & -2.114 \\
\hline 11 & 0.373 & 0.274 & -0.987 & 0.427 & 0.268 & -0.850 & 0.319 & 0.266 & -1.142 \\
\hline 12 & 0.057 & 0.110 & -2.858 & 0.059 & 0.109 & -2.834 & 0.057 & 0.109 & -2.868 \\
\hline 13 & 0.086 & 0.105 & -2.450 & 0.090 & 0.104 & -2.412 & 0.085 & 0.104 & -2.465 \\
\hline 14 & 0.040 & 0.038 & -3.214 & 0.040 & 0.038 & -3.209 & 0.040 & 0.038 & -3.214 \\
\hline 15 & 0.041 & 0.100 & -3.187 & 0.042 & 0.100 & -3.168 & 0.041 & 0.100 & -3.199 \\
\hline 16 & 0.042 & 0.100 & -3.182 & 0.043 & 0.100 & -3.158 & 0.041 & 0.100 & -3.187 \\
\hline 17 & 0.049 & 0.109 & -3.008 & 0.051 & 0.109 & -2.974 & 0.049 & 0.109 & -3.008 \\
\hline 18 & 0.041 & 0.141 & -3.187 & 0.042 & 0.141 & -3.170 & 0.039 & 0.120 & -3.244 \\
\hline 19 & 0.040 & 0.092 & -3.211 & 0.041 & 0.092 & -3.206 & 0.027 & 0.066 & -3.605 \\
\hline 20 & 0.064 & 0.082 & -2.755 & 0.064 & 0.082 & -2.746 & 0.063 & 0.082 & -2.761 \\
\hline 21 & 0.094 & 0.171 & -2.364 & 0.096 & 0.171 & -2.339 & 0.070 & 0.140 & -2.659 \\
\hline 22 & 0.094 & 0.239 & -2.366 & 0.098 & 0.238 & -2.328 & 0.046 & 0.145 & -3.077 \\
\hline 23 & 0.091 & 0.067 & -2.395 & 0.091 & 0.067 & -2.393 & 0.069 & 0.059 & -2.675 \\
\hline 24 & 0.038 & 0.083 & -3.268 & 0.037 & 0.078 & -3.310 & 0.018 & 0.046 & -4.017 \\
\hline 25 & 0.125 & 0.092 & -2.079 & 0.118 & 0.088 & -2.137 & 0.046 & 0.063 & -3.073 \\
\hline
\end{tabular}




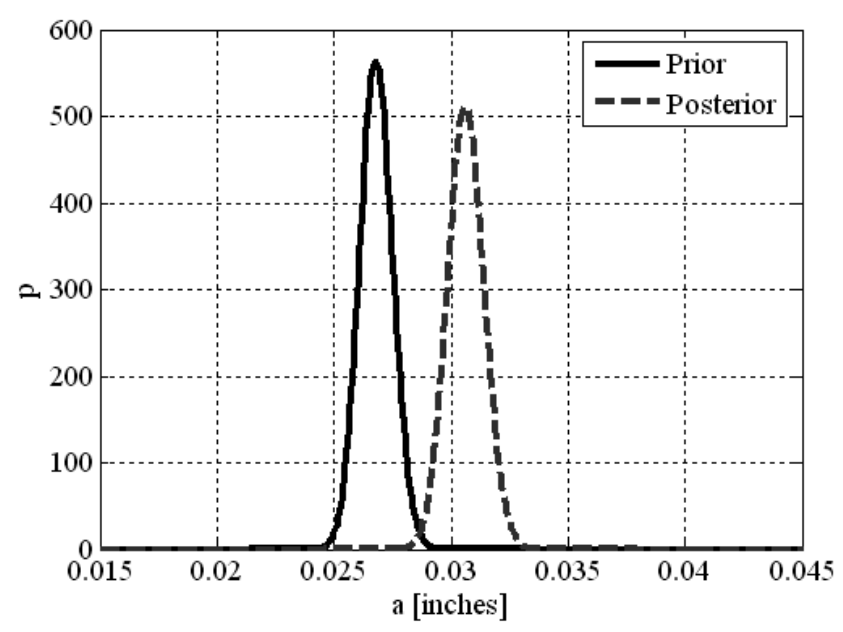

Figure 6.3 Bayesian Updating Results.

If one uses the estimated parameters and the mean of the posterior distribution at $F L E=j \%$, a line parallel to the "prior" line shown in Figure 6.1 describes the posterior mean crack size growth as a function of FLE.

A comparison of the results was carried out using the MCMC methodology discussed in Chapter 5. The Bayesian approach based on the MCMC methodology requires a number of components: the likelihood, the data or evidence and parameter distributions. The methodology estimate the posterior parameter distribution $p(\theta \mid D)$ implied by the observed data $D$. A prior distribution $p(\theta)$ represents any known information regarding the parameters before $D$ is observed; in this case, a noninformative prior is used to assign equal probability density to all combinations of parameter values $k, n$, and $\sigma$. The likelihood distribution is represented by the Equation 5.12. With an increasing length of sequence data, $p(\theta \mid D)$ approaches a normal distribution centered on the MLE of the parameters, independently of the prior distribution. 
A Markov chain with stationary distribution $p(\theta \mid D)$ is used to generate the sample $\left(\theta_{1}, \theta_{2}, \ldots, \theta_{\mathrm{i}}\right)$. At each step of this chain, we update a single parameter $\theta_{\mathrm{i}}$. After a large number of updates, the sample of realizations is effectively drawn from $p(\theta \mid D)$.

Once the parameters, $k, n, \sigma$ are obtained, the lognormal distribution of crack size at $F L E_{j \%}$ for each aircraft is calculated using the following equations.

$$
f\left(a \mid F L E_{j \%}\right)=\iint_{k} \int_{n} \frac{1}{a \cdot \sigma_{a} \sqrt{2 \pi}} \exp \left[-\frac{1}{2}\left(\frac{\operatorname{Ln}(a)+\operatorname{Ln}(k)-n \operatorname{Ln}(F L E \quad j \%)}{\sigma_{a}}\right)^{2}\right]
$$

Table 6.2 shows the results of the updating the 25 aircrafts.

Table 6.2 Lognormal distribution parameters at $F L E_{j \%}$ for each aircraft - MCMC.

\begin{tabular}{|c|c|c|c|}
\hline Aircraft & $\mu_{\text {up }}$ & $\sigma_{\text {up }}$ & $\mu_{\text {upLn }}$ \\
\hline 1 & 0.059 & 0.566 & -2.818 \\
\hline 2 & 0.039 & 0.202 & -3.222 \\
\hline 3 & 0.043 & 1.057 & -3.138 \\
\hline 4 & 0.090 & 0.247 & -2.403 \\
\hline 5 & 0.040 & 1.548 & -3.220 \\
\hline 6 & 0.089 & 0.536 & -2.413 \\
\hline 7 & 0.039 & 1.290 & -3.220 \\
\hline 8 & 0.178 & 0.433 & -1.721 \\
\hline 9 & 0.040 & 0.533 & -3.215 \\
\hline 10 & 0.125 & 0.545 & -2.078 \\
\hline 11 & 0.376 & 0.507 & -0.978 \\
\hline 12 & 0.055 & 0.554 & -2.887 \\
\hline 13 & 0.093 & 0.392 & -2.375 \\
\hline 14 & 0.039 & 0.026 & -3.220 \\
\hline 15 & 0.040 & 0.334 & -3.217 \\
\hline 16 & 0.039 & 0.322 & -3.222 \\
\hline 17 & 0.053 & 0.504 & -2.927 \\
\hline 18 & 0.039 & 1.550 & -3.222 \\
\hline 19 & 0.040 & 0.263 & -3.218 \\
\hline 20 & 0.089 & 0.314 & -2.413 \\
\hline 21 & 0.170 & 1.056 & -1.772 \\
\hline 22 & 0.173 & 0.537 & -1.751 \\
\hline 23 & 0.066 & 0.315 & -2.716 \\
\hline 24 & 0.040 & 0.207 & -3.220 \\
\hline 25 & 0.145 & 0.335 & -1.929 \\
\hline
\end{tabular}




\subsection{Posterior $p d f$ for $F L E=100 \%$}

This step is carried out in order to improve the estimation of the initial crack distribution at $100 \%$ FLE by taking into account the evidence observed. It consists of first updating the conditional $p d f$ of the prior crack size (at $F L E=j \%$ ) and extrapolating the resulting posterior $p d f$ (at $F L E=j \%$ ) back to $F L E=100 \%$, thus calculating the posterior crack size distribution at $F L E=100 \%$. However, it is important to note that multiple combinations of the initial model parameters and initial crack size need to be determined in order to reach the mean of posterior distribution where $F L E=j \%$. Figure 6.4 illustrates the process used to obtain these combinations. Initial random values of the parameters and initial crack size are sampled from their distributions and used in the crack growth model. If the result is equal or within the range of the target resulting from the updating process, the initial values are considered as a combination that meet the requirement, and a new combination is tested. 


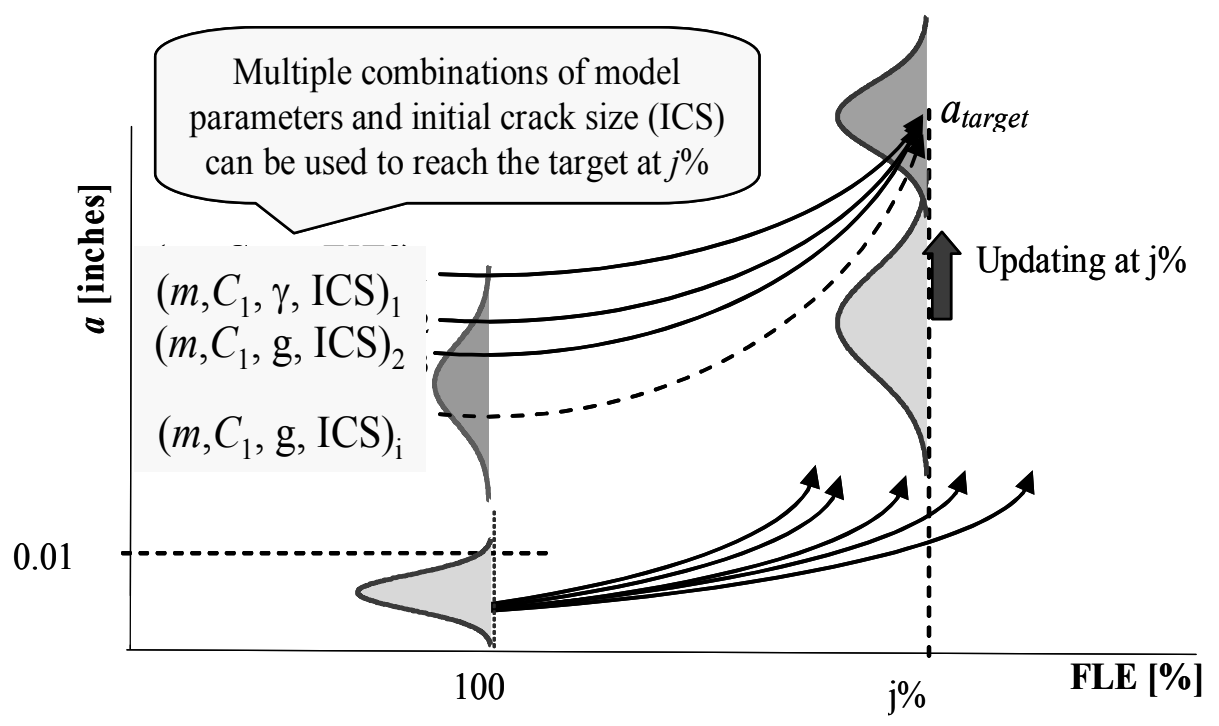

Figure 6.4 Updating Prior Crack Size Distributions.

Figure 6.5 shows a simplified algorithm used in the PCGROW code for the Walker model. The same algorithm was used for the Forman and Closure models. It is important to recall that a table lockup was used for the crack closure model; therefore, only three parameters are updated for this model. 


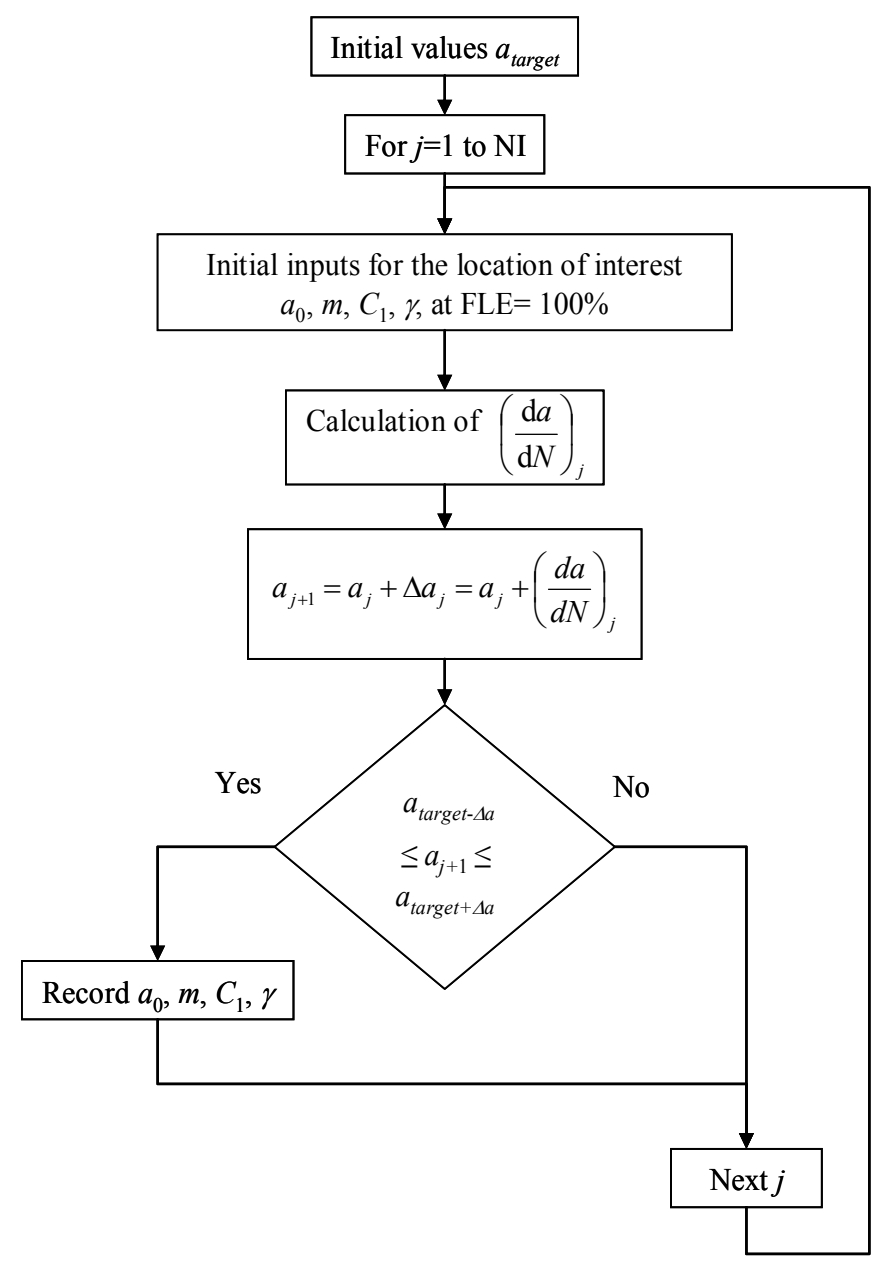

The $\Delta a$ used in the calculations is based on the $2.5 \%$ and $97.5 \%$ from the Winbugs results.

Figure 6.5 Algorithm used in PCGROW code for the parameters updating.

From the Monte Carlo Simulations, any number of parameter estimates and initial crack size may be calculated. From these trial estimates, a distribution for each parameter and initial crack size can be estimated. Applying the same methodology for the rest of the aircrafts, we will obtain $25 p d f s$ for each parameter. See Figure 6.6. 

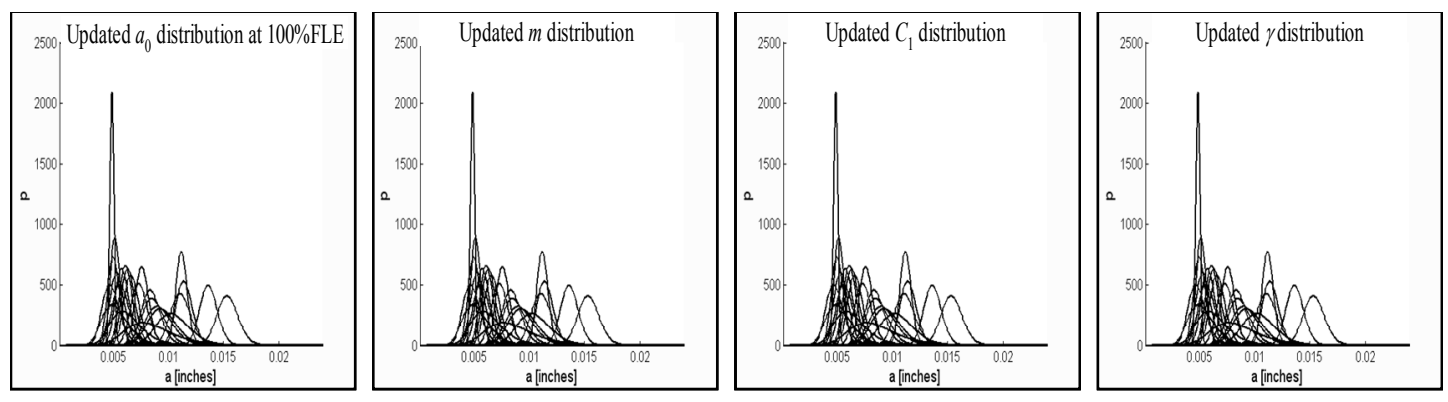

Figure 6.6 Updated distributions at 100\% FLE.

To obtain the combined updated initial crack length distribution, a linear arithmetic weighted approach is used, by assigning equal weight to each posterior initial crack size probability. As such

$$
w=w_{1} \cdot p_{1}(\Delta a)+w_{2} \cdot p_{2}(\Delta a)+\ldots . w_{\mathrm{n}} \cdot p_{\mathrm{n}}(\Delta a)
$$

where $w$ is the weight $\left(w_{\mathrm{i}}=1 / \mathrm{na}\right)$ and $p(\Delta a)$ is the probability that a random initial crack will have a size in the interval $\Delta a$, and na is the number of aircrafts analyzed (in this case na=25).

The following paragraphs show the results for the initial crack size and the model parameters after updating at $100 \%$ FLE.

\subsubsection{Posterior Initial Crack Size $p d f$ for $F L E=100 \%$}

Each aircraft analyzed yields a posterior crack size distribution corresponding to $F L E=100 \%$. Hence, for the 25 aircraft studied, the analysis estimates 25 corresponding posterior crack size distributions at $F L E=100 \%$. Combination of these 25 posterior distributions leads to an estimate of "fleet" crack size distribution at $F L E=100 \%$. This distribution may be used for any aircraft in the fleet.

Figures 6.8- 6.10 show the combined $p d f$ for the 25 aircraft according to Equation 
6.9. Because this $p d f$ is multimodal, assuming that the resulting combined distribution should be smooth (i.e., only one underlying failure mechanism of strain-caused crack imitation is active), the combined distribution is "smoothed" by fitting a lognormal distribution developed by way of moment matching, which is a technique for constructing estimators of the parameters that is based on matching the sample moments with the corresponding distribution moments (see Figure 6.7)[124 ].

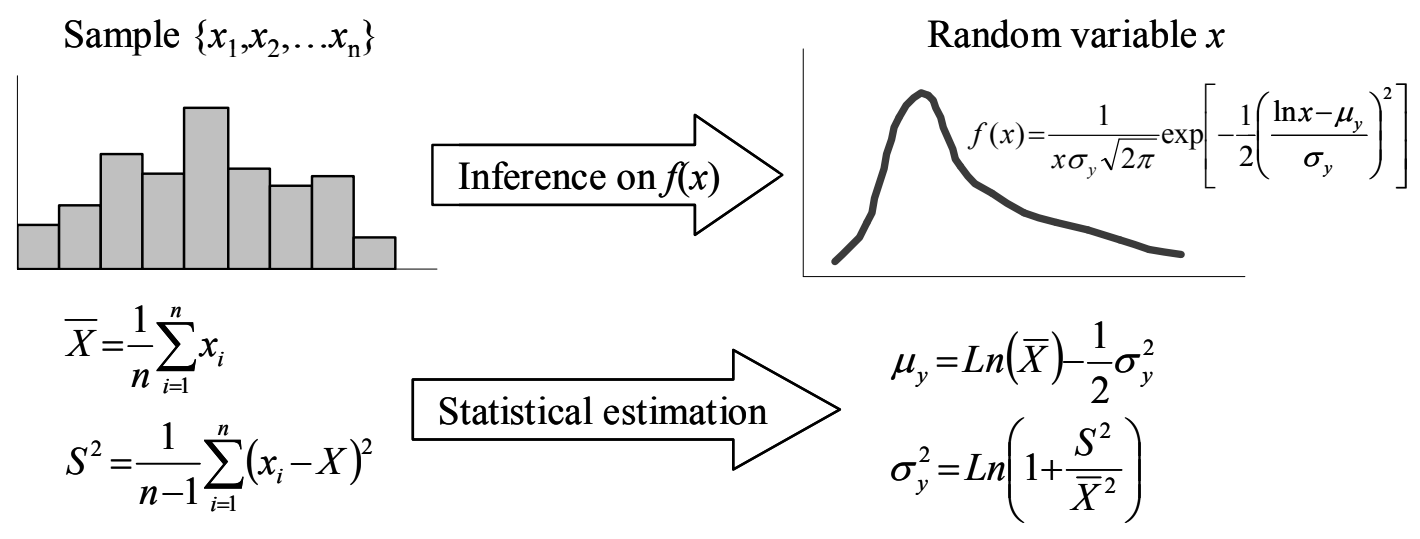

Figure 6.7 Method of moments. 


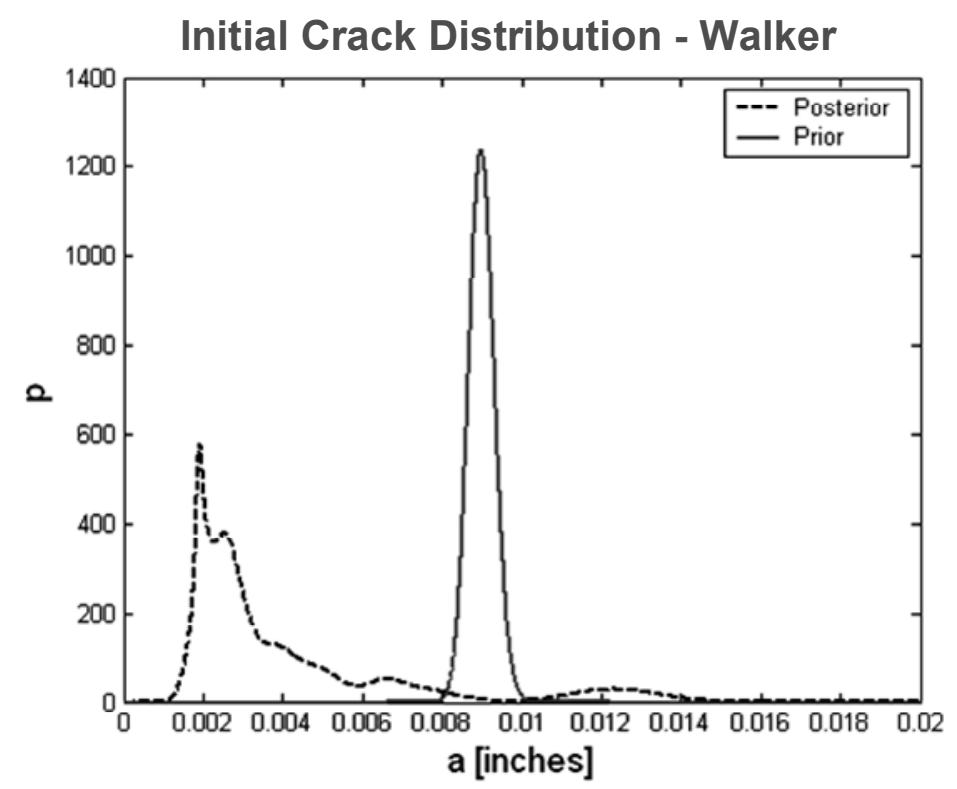

Figure 6.8 Prior \& Posterior crack size $p d f, F L E=100 \%$ - Walker model.

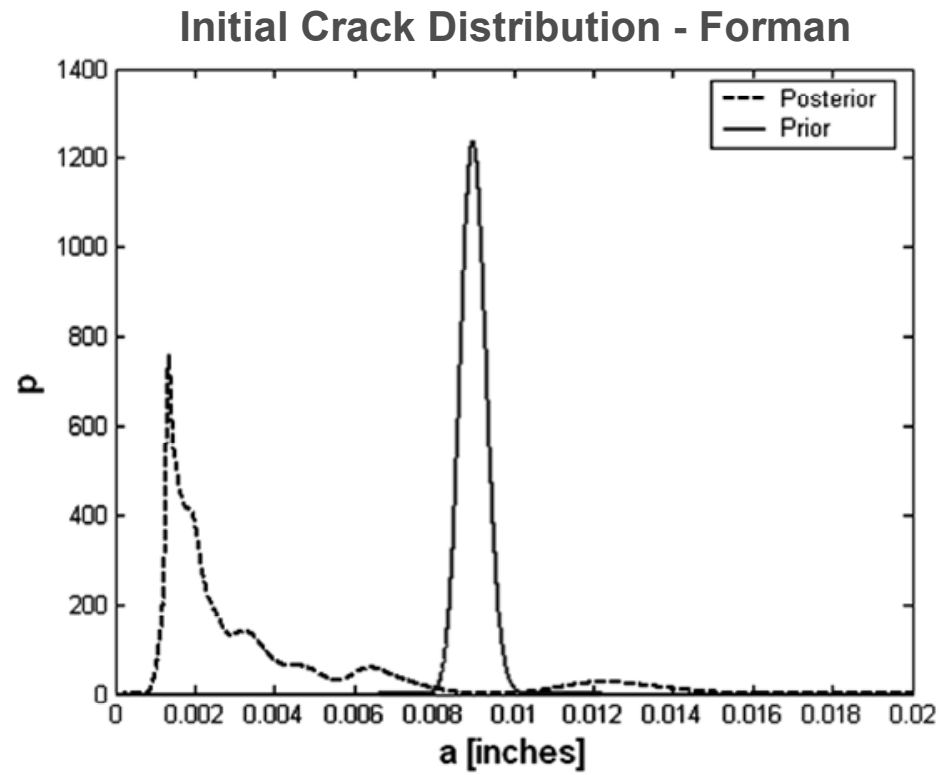

Figure 6.9 Prior \& Posterior crack size $p d f, F L E=100 \%$ - Forman model. 


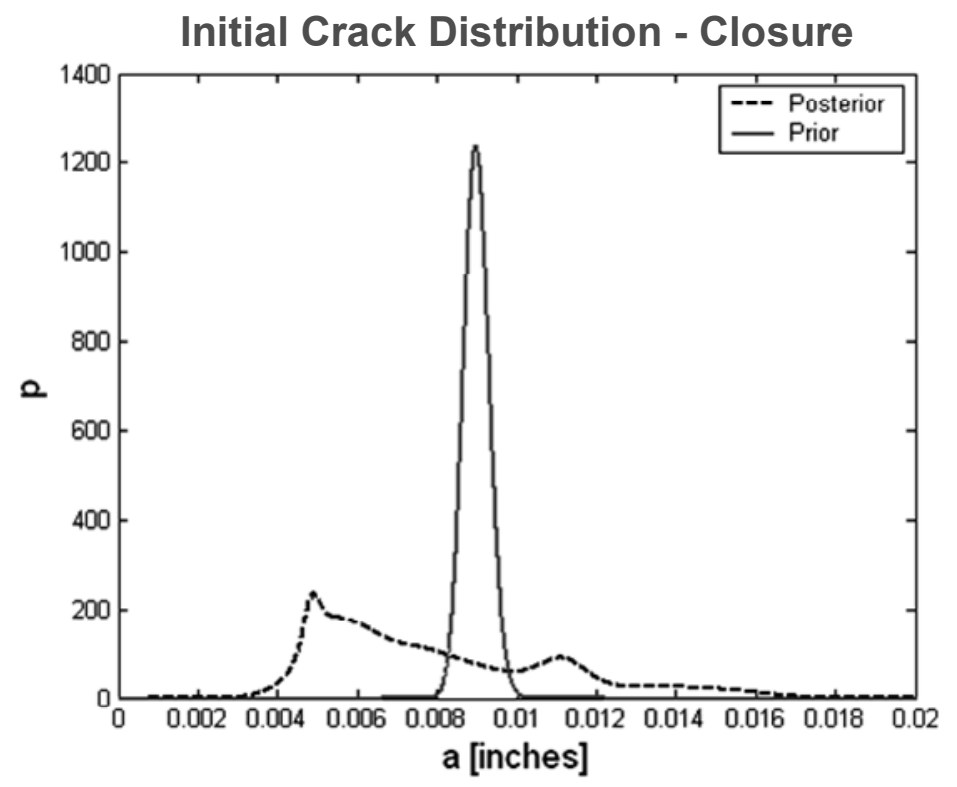

Figure 6.10 Prior \& Posterior crack size $p d f, F L E=100 \%$ - Closure model.

Figures $6.11-6.13$ show the resulting smooth lognormal $p d f$ of the initial crack size distribution for the aircraft population, based on the 25 sample data points for each model.

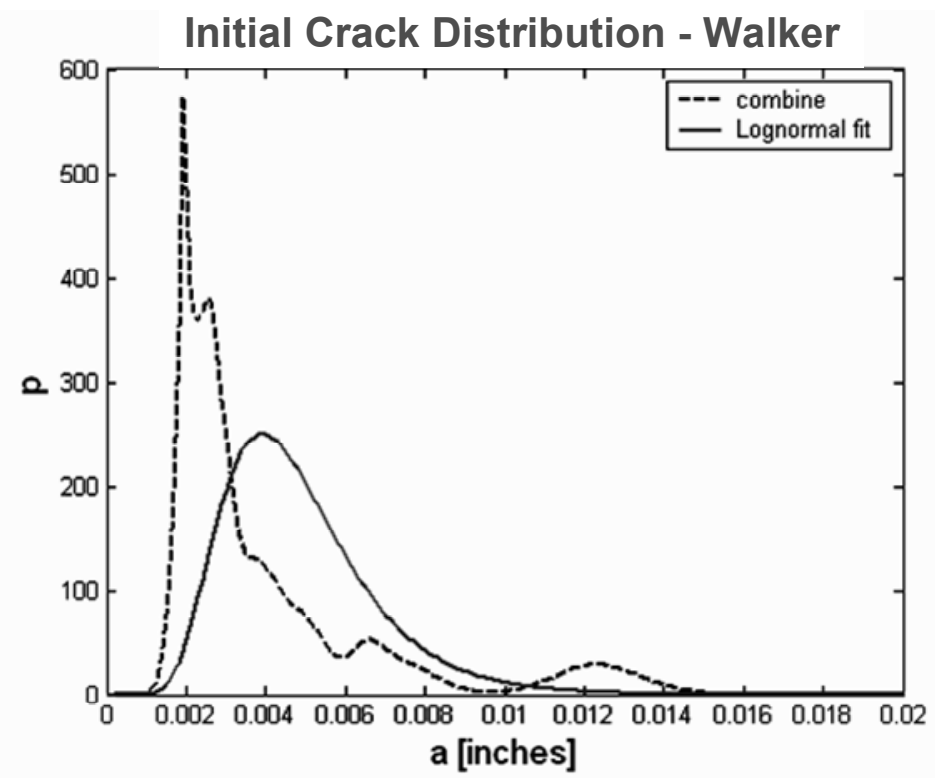

Figure 6.11 Posterior versus smooth crack size $p d f, F L E=100 \%$ - Walker model 


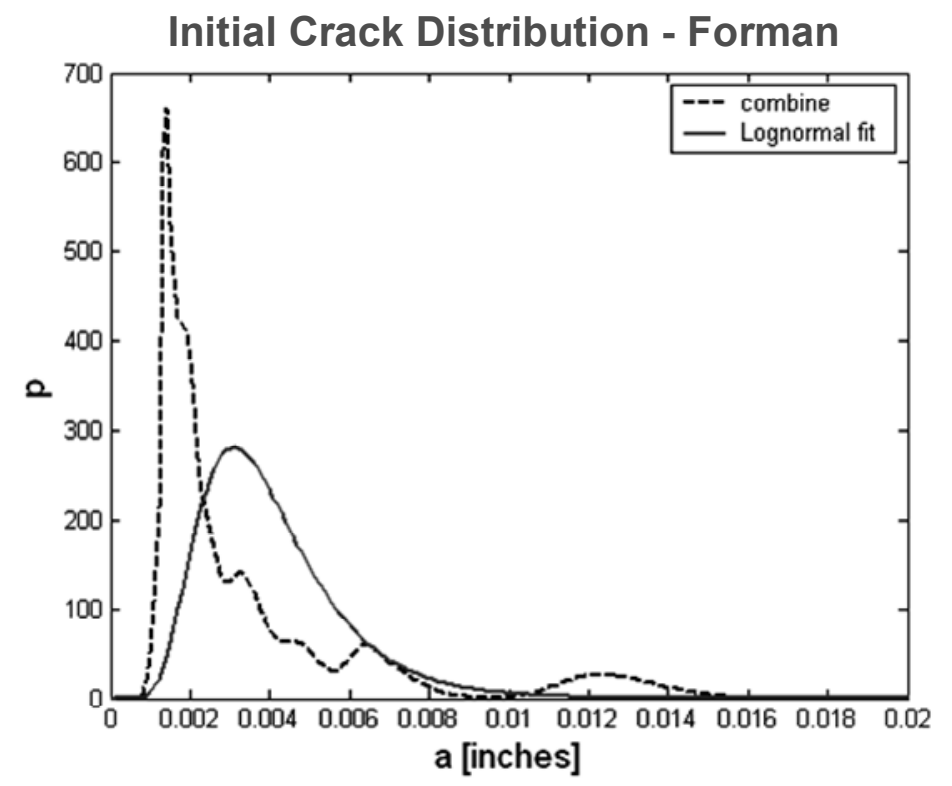

Figure 6.12 Posterior versus smooth crack size $p d f, F L E=100 \%$ - Forman model

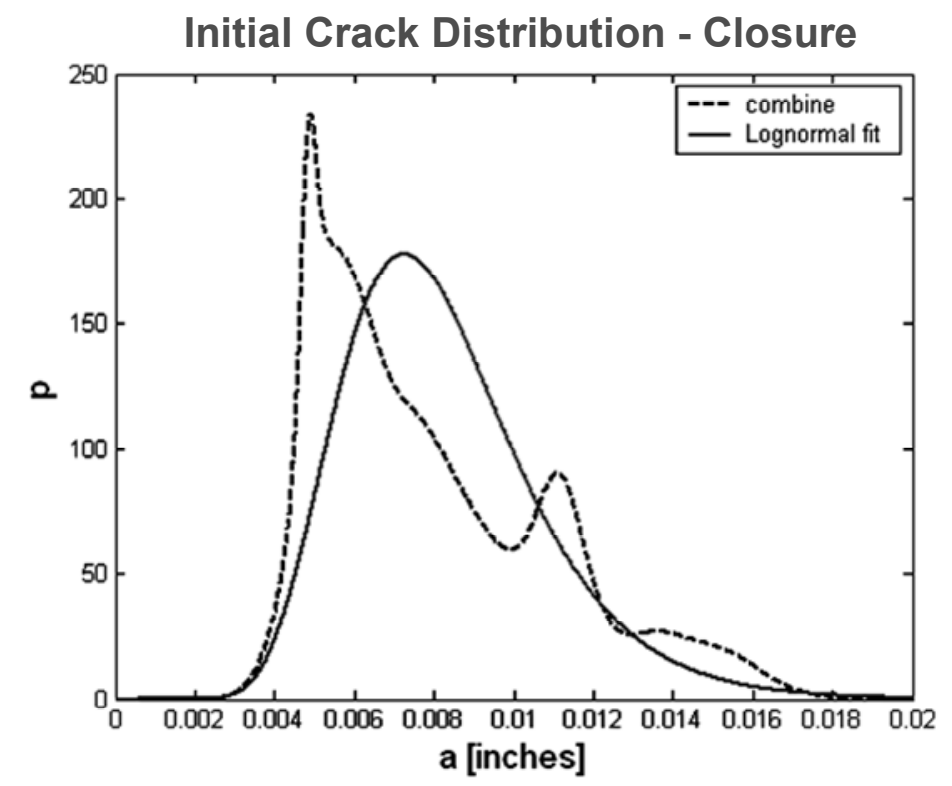

Figure 6.13 Posterior versus smooth crack size pdf, $F L E=100 \%$ - Closure model. 


\subsubsection{Posterior Model Parameters}

Like the initial crack size, a combination of the 25 posterior distributions of parameters leads to a new distribution, which can be taken as representative of the fleet initial crack size. As $p d f$ mentioned in section 6.2, from the Monte Carlo results, a distribution for each parameter can be estimated. Applying the same methodology for the rest of the aircraft, we will obtain $25 \mathrm{pdfs}$ for each parameter. Figures $6.14-6.16$ show the $p d f s$ for the 25 aircrafts and the prior and posterior $p d f$ at $100 \%$ FLE for the Walker model, Figures 6.16 - 6.18 for the Forman model, and Figures 6.20 - 6.22 for the Closure model.
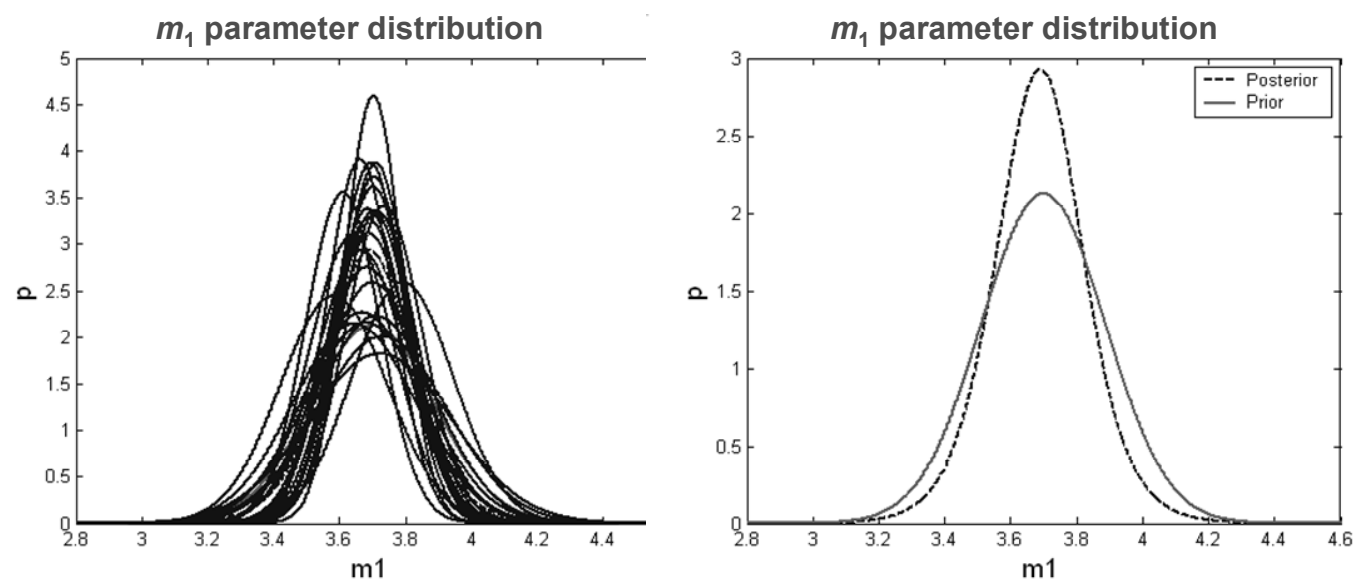

Figure 6.14 $m_{1}$ parameter. $p d f, F L E=100 \%$. 

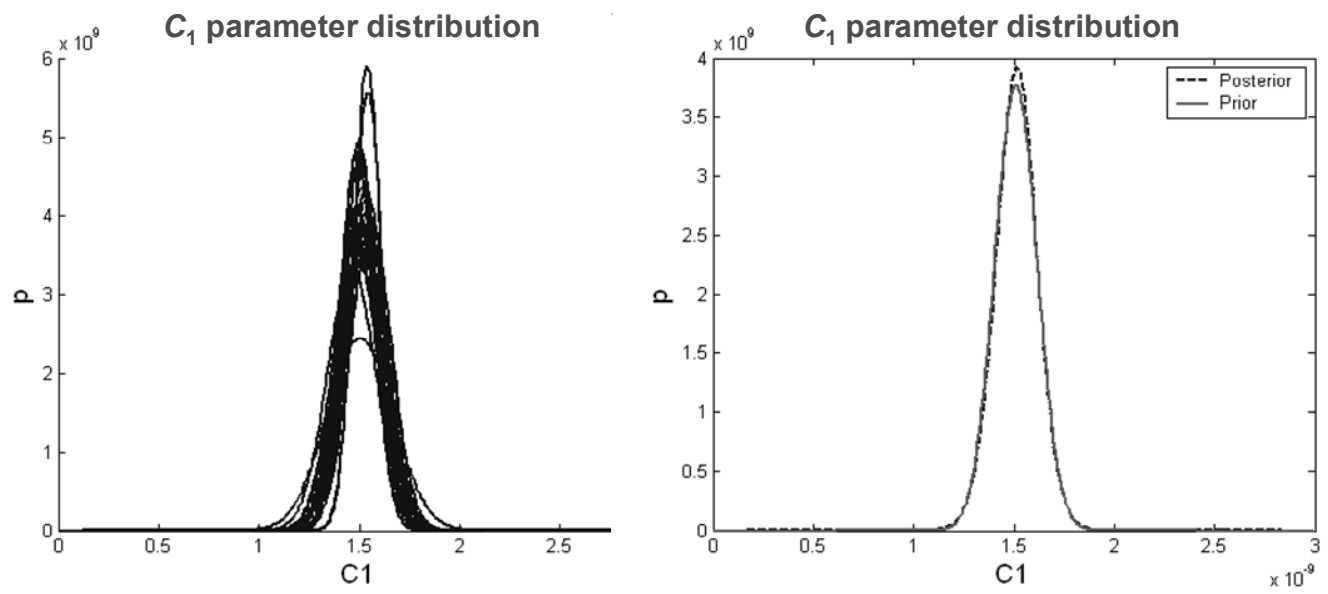

Figure 6.15 $C_{1}^{*}$ parameter $p d f, F L E=100 \%$.
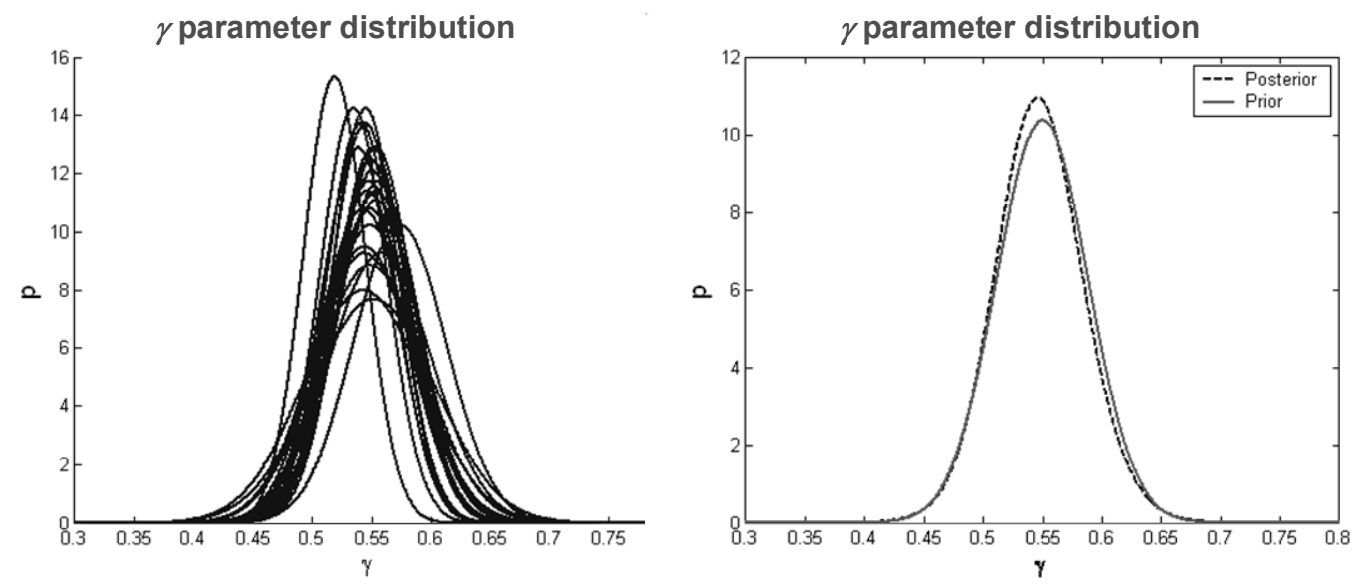

Figure 6.16 $\gamma$ parameter. $p d f, F L E=100 \%$. 

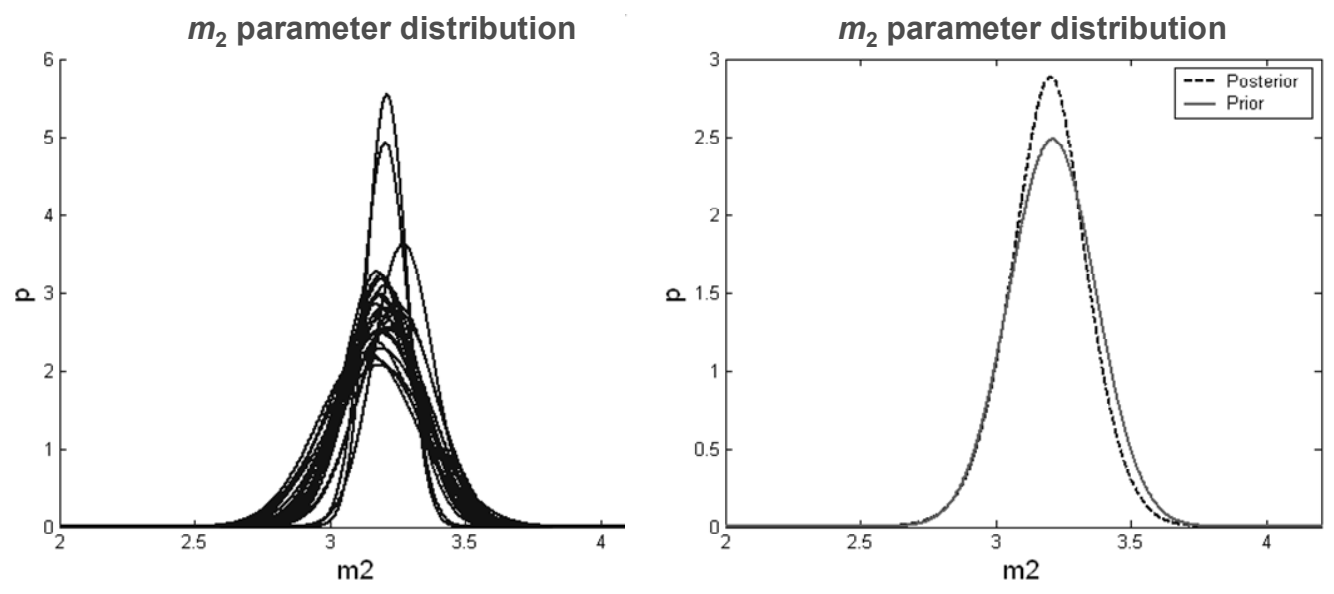

Figure 6.17 $m_{2}$ parameter. $p d f, F L E=100 \%$.
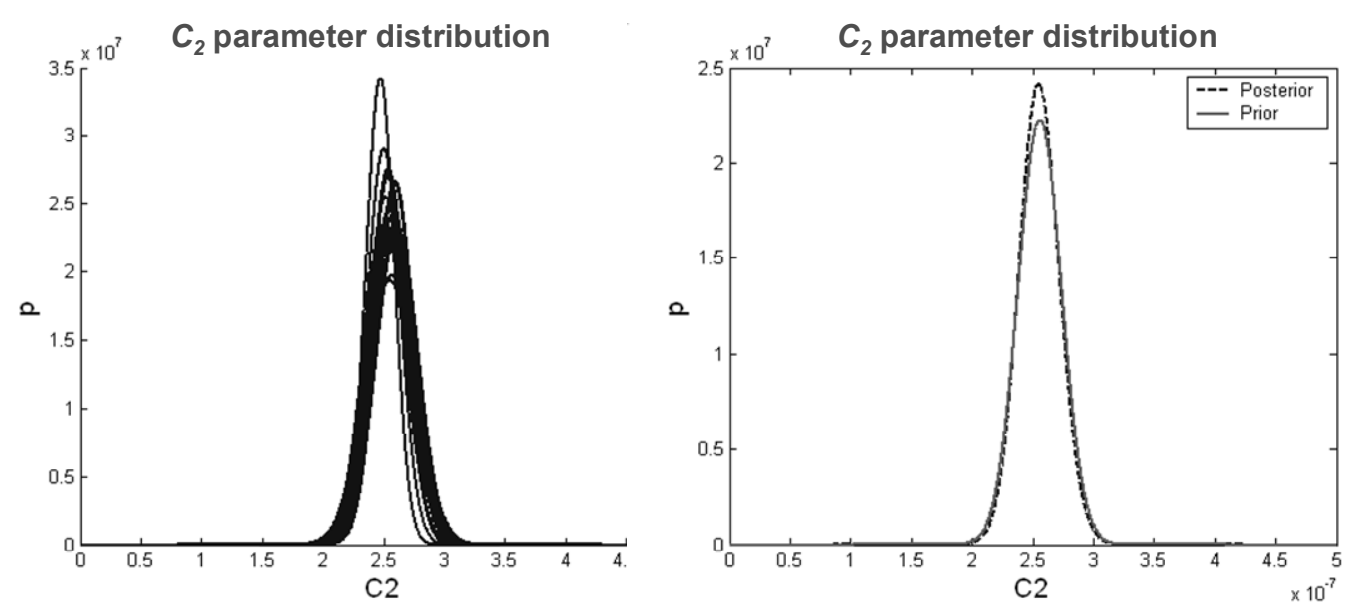

Figure 6.18 $C_{2}$ parameter. $p d f, F L E=100 \%$. 

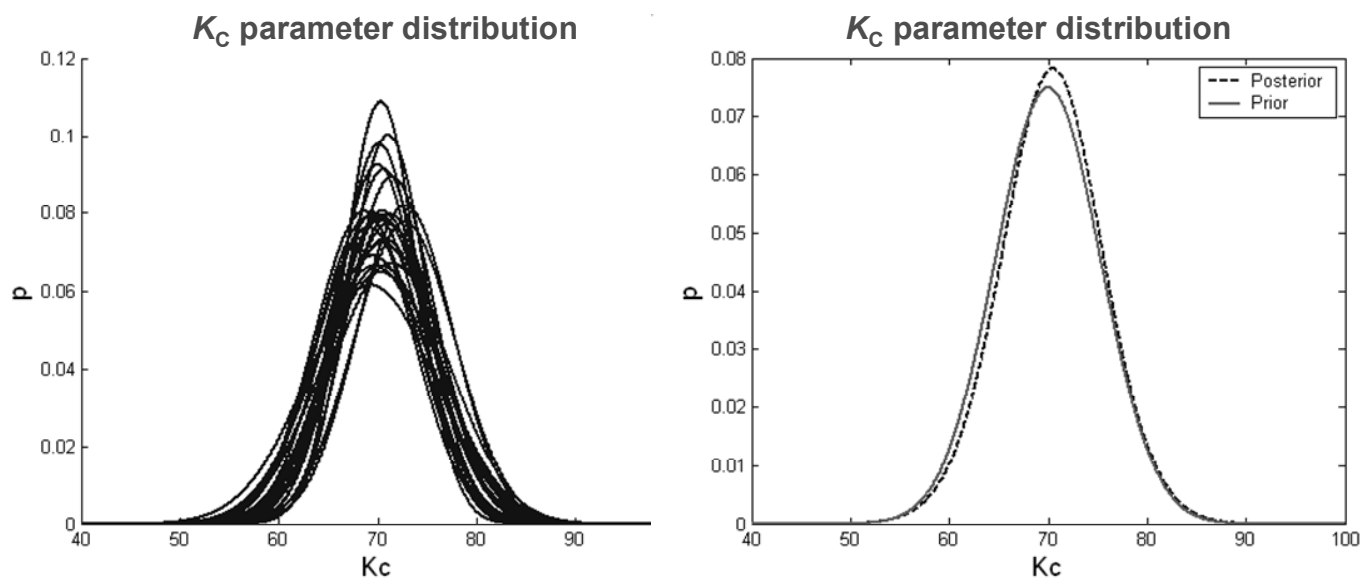

Figure 6.19 $K_{\mathrm{C}}$ parameter. $p d f, F L E=100 \%$.
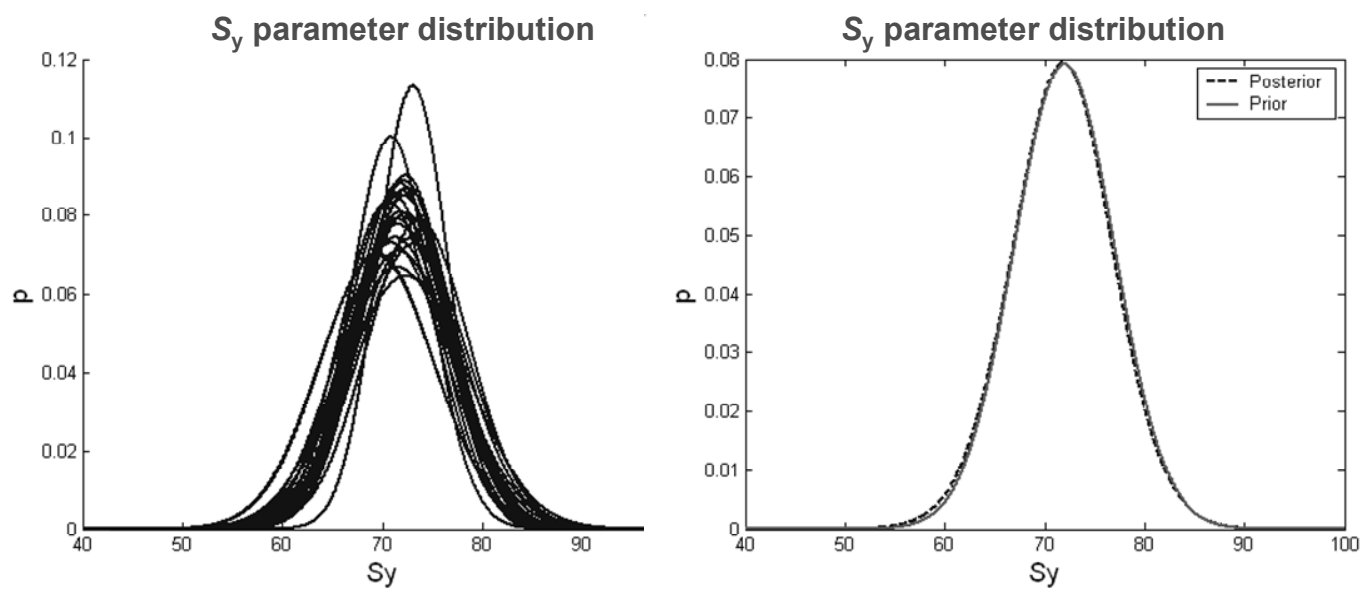

Figure 6.20 $S_{\mathrm{y}}$ parameter. $p d f, F L E=100 \%$. 

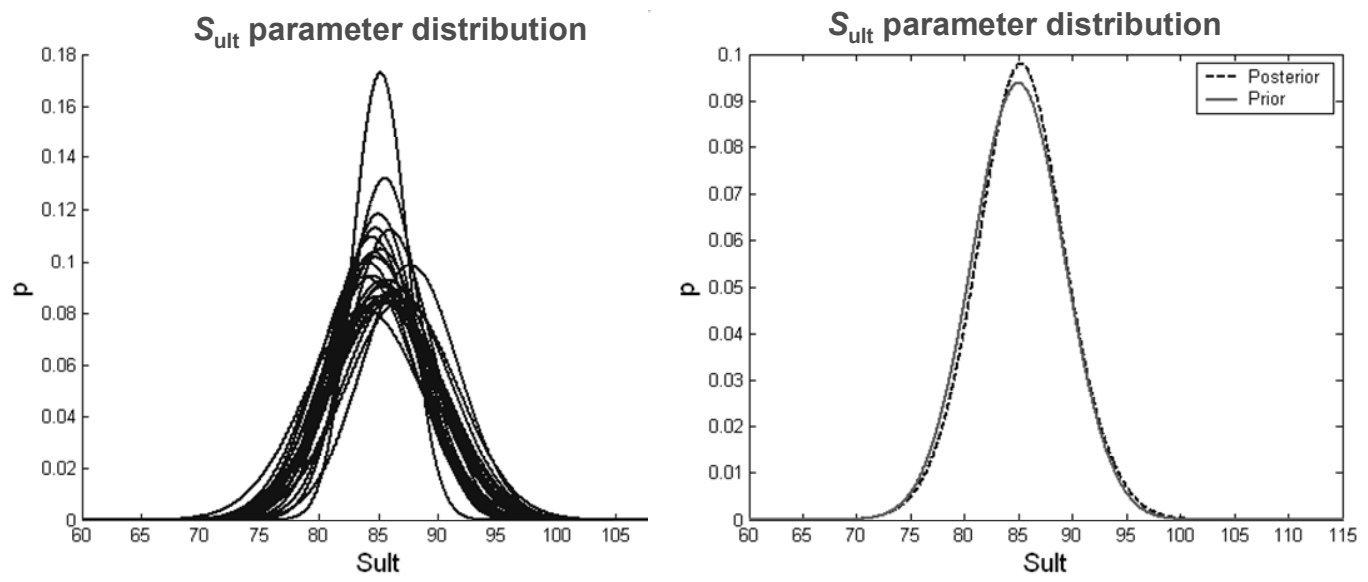

Figure 6.21 $S_{\text {ult }}$ parameter. $p d f, F L E=100 \%$.
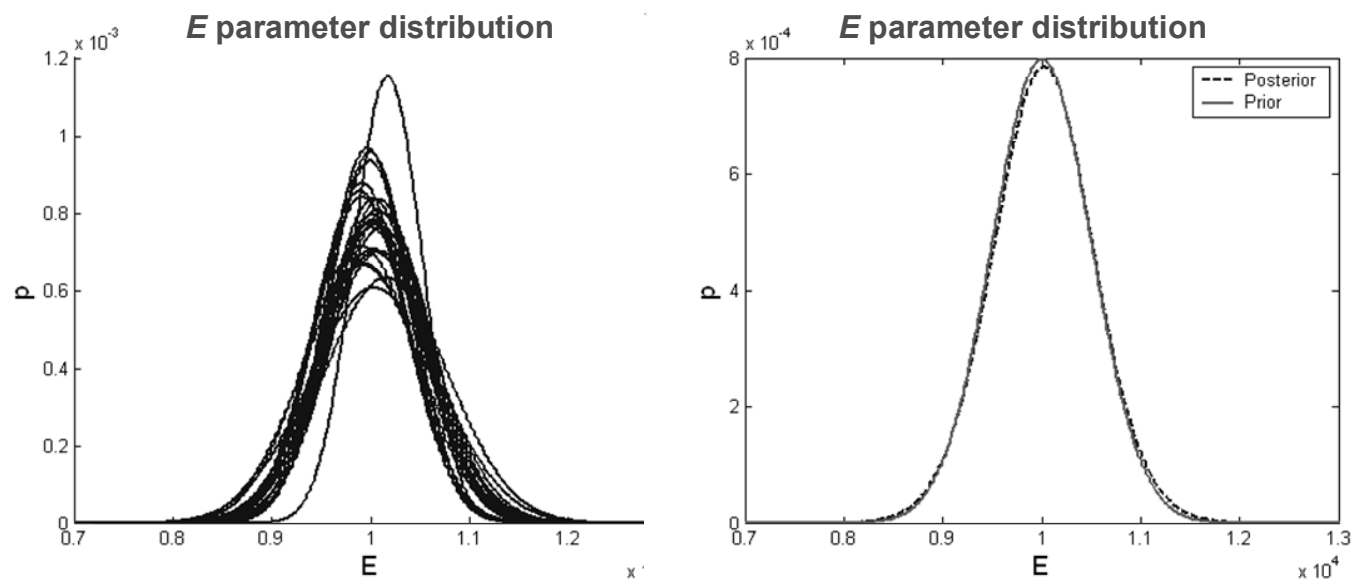

Figure 6.22 $E$ parameter. $p d f, F L E=100 \%$.

If the posterior $p d f s$ are multimodal, then keeping the same assumption as the initial crack size, the resulting combined distribution should be "smoothed" by fitting a normal distribution (developed by way of moment matching). Table 6.3 shows the prior and posterior values. 
Table 6.3 Model parameters updating.

\begin{tabular}{|c|c|c|c|c|c|c|}
\hline \multirow{2}{*}{ Parameter } & \multicolumn{3}{|c|}{ Prior } & \multicolumn{3}{c|}{ Posterior } \\
\cline { 2 - 7 } & mean & SD & CV & mean & SD & CV \\
\hline$m_{1}$ & 3.70 & 0.19 & $5.00 \%$ & 3.69 & 0.14 & $3.73 \%$ \\
\hline$C_{1}^{*}$ & $1.51 \mathrm{E}-09$ & $1.06 \mathrm{E}-10$ & $7.00 \%$ & $1.53 \mathrm{E}-09$ & $1.03 \mathrm{E}-10$ & $6.71 \%$ \\
\hline$\gamma$ & 0.55 & 0.04 & $7.00 \%$ & 0.54 & 0.04 & $6.73 \%$ \\
\hline$m_{2}$ & 3.21 & 0.16 & $5.00 \%$ & 3.19 & 0.14 & $4.39 \%$ \\
\hline$C_{2}$ & $2.56 \mathrm{E}-07$ & $1.79 \mathrm{E}-08$ & $7.00 \%$ & $2.54 \mathrm{E}-07$ & $1.66 \mathrm{E}-08$ & $6.54 \%$ \\
\hline$K_{\mathrm{c}}$ & 70.00 & 4.9 & $7.00 \%$ & 70.50 & 5.10 & $7.23 \%$ \\
\hline$S_{\mathrm{y}}$ & 72.00 & 5.04 & $7.00 \%$ & 71.60 & 5.01 & $7.00 \%$ \\
\hline$S_{\text {ult }}$ & 85.00 & 4.25 & $5.00 \%$ & 85.30 & 4.08 & $4.78 \%$ \\
\hline$E$ & 10000.00 & 500.00 & $5.00 \%$ & 10030.00 & 506.00 & $5.04 \%$ \\
\hline
\end{tabular}

\subsubsection{Posterior Initial Crack Size $p d f$ for $F L E=100 \%$ - MCMC}

The same methodology used in section 6.2.1 was applied to obtain the initial crack size through the use of the MCMC approach. Figures $6.23-6.25$ show the resulting smooth $p d f$ of the initial crack size distribution for the aircraft population, based on the 25 sample data points for each model. 


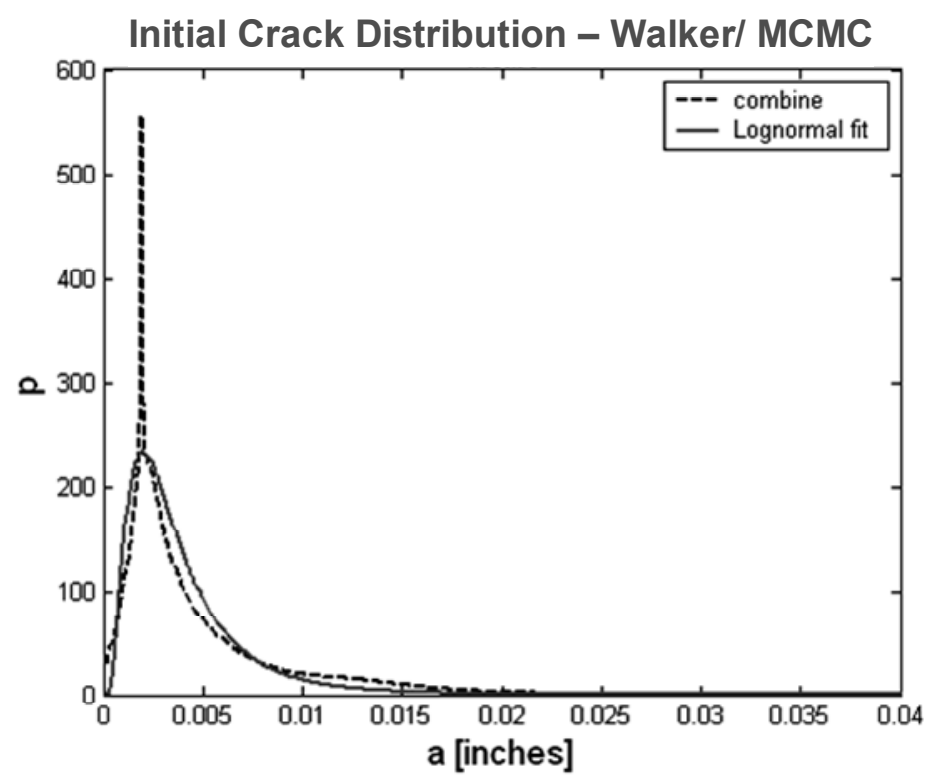

Figure 6.23 Posterior versus smooth crack size $p d f, F L E=100 \%$ - Walker model, MCMC approach.

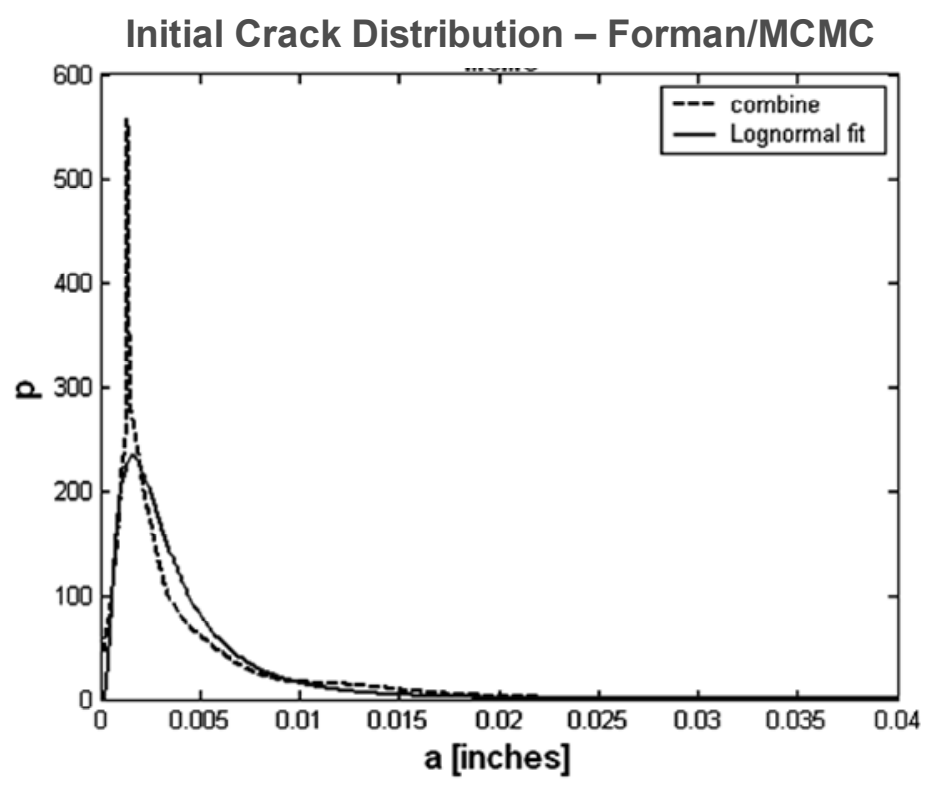

Figure 6.24 Posterior versus smooth crack size $p d f, F L E=100 \%$ - Forman model, MCMC approach. 


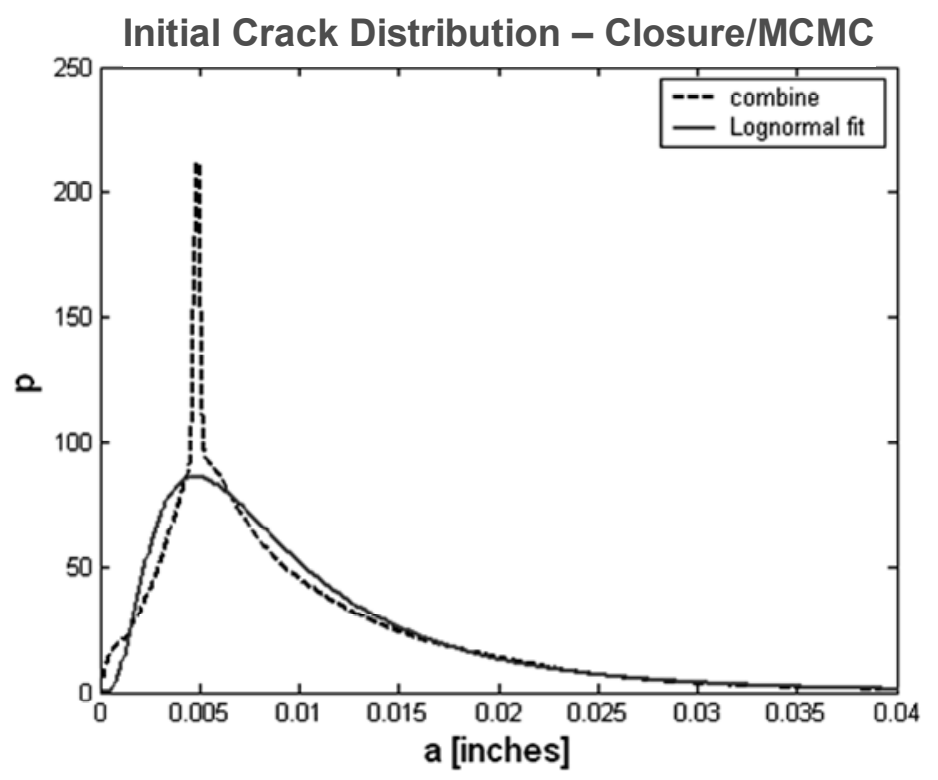

Figure 6.25 Posterior versus smooth crack size $p d f$, FLE $=100 \%$ - Closure model,

MCMC approach.

The differences between Conjugate and MCMC approach will be discussed in Chapter 7.

\subsection{Forecasting Probability of Failure for a Specific $F L E_{j \%}$}

Given that the updated combined initial crack size distribution represents the total population of the aircrafts, and assuming no information exits other than the loads experienced by aircrafts to reach its present $F L E_{j \%}$, a Monte Carlo simulation might be used to project crack size distribution that results from additional flight hours $(\Delta \mathrm{FH})$ beyond $F L E_{j \%}$.

The procedure takes the recorded loads for this aircraft sequentially to reach $F L E_{j \%}$. Once reached, the loads are sampled randomly to grow cracks beyond $F L E_{\mathrm{j} \%}$ until they reach the desired $\triangle F H$. The resulting distribution is used to determine the 
probability that it exceeds critical crack size for failure. The critical crack size is calculated using the applicable fracture toughness $K_{C}$ associated with the dome nut hole. Figure 6.26 illustrates the process.

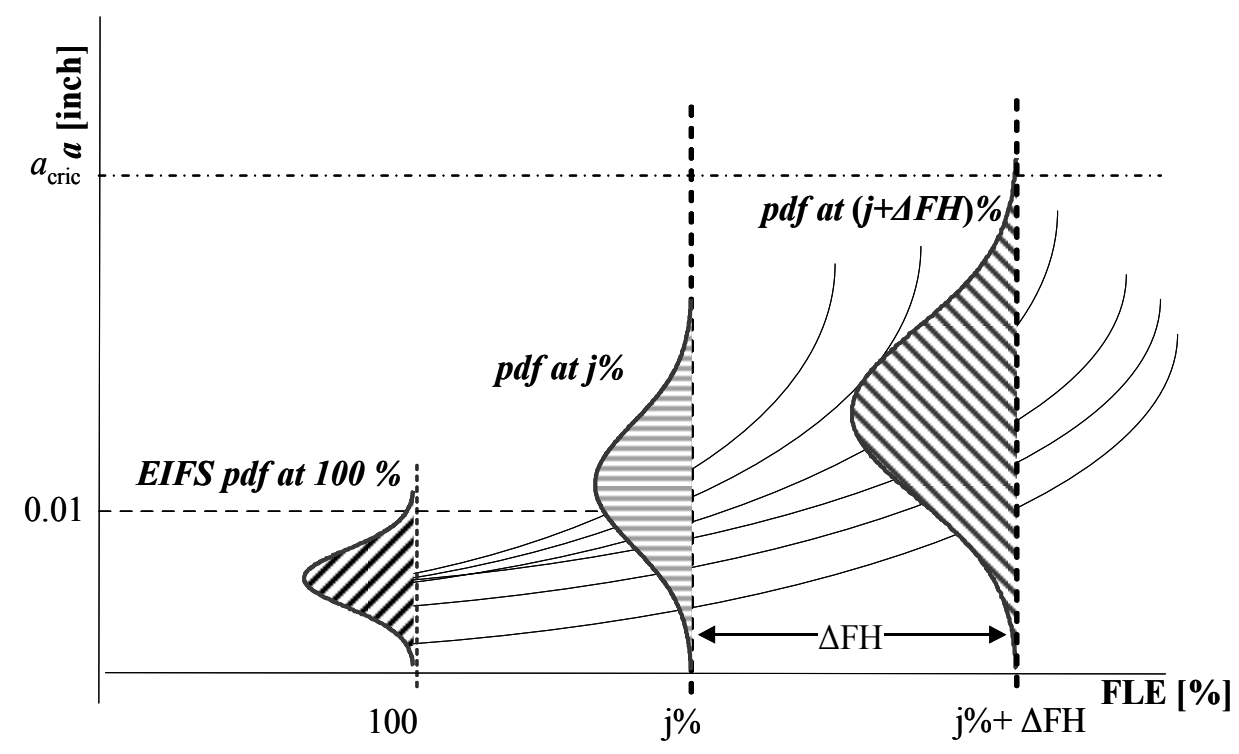

Figure 6.26 Forecasting process for a specific $F L E_{j \%}$.

\subsection{Decision Making Methodology}

Uncertainties in this research arise when dealing with the variables that affect the damage crack growth models, and when dealing with the different outputs that result when different damage crack growth model are applied. Fleet managers responsible for maintaining the existing aircrafts have to face this, in order to ensure the structural integrity of the aircraft.

In this research, we propose a decision making process based on three elements, which are explained in the following paragraphs. 
First one must determine the maximum crack size or damage that can be repaired or restored at a reasonable cost. A cost benefit analysis needs to be done based on the maintenance history of the fleet.

Second element is to define the probability of crack size that the decision maker is willing to accept (or actual crack size he/she accepts).

Third factor to address is characterization of the uncertainties of the crack growth models. There is no universally accepted model that represents the phenomenon of fatigue. Therefore, it is up to the decision maker to determine which model represents the best one for the decision making. As mentioned in Chapter 1, this implies a trade-off between precision and the uncertainty propagation due to the number of parameters involved in the model.

Since the work of Paul Paris in 1961, who postulated his law under conditions of constant amplitude loading and long cracks, a great number of different models have been proposed to handle the cases that do not meet the Paris law conditions. Fatigue life prediction remains very much an empirical science at present [125]. Model uncertainty arises when different models, using the same set of data lead to different predictions. The proliferation of models would appear to suggest that the essential physics of fatigue-crack growth is not completely captured by only one model.

Although model uncertainty is a field that requires more research, it is possible to make a classification based on the prediction of a property of interest: single model and multiple models methods [126]. Figure 6.27 illustrates this classification.

One model case is out of this scope; therefore the analysis in this research is focus on the case of multiple models where the same data, information and knowledge is used 
to develop a model, but with different assumptions. The damage crack growth estimation developed in this work meets the conditions because the three models assume totally different phenomena of crack growth due to fatigues, and they may satisfy the same data and information available. In this case, the averaging approach (used when the assumptions in developing the model are the same) is meaningless, because for the given problem of interest, only one of these models may be appropriate. The characterization of epistemic uncertainty of the model accuracy can only be done separately for each model, and only qualitatively (or semi-quantitatively) explain the goodness of each model. It becomes the final decision maker's partiality (e.g., fleet manager's decision as to which of the model output he/she prefers to use) [127].

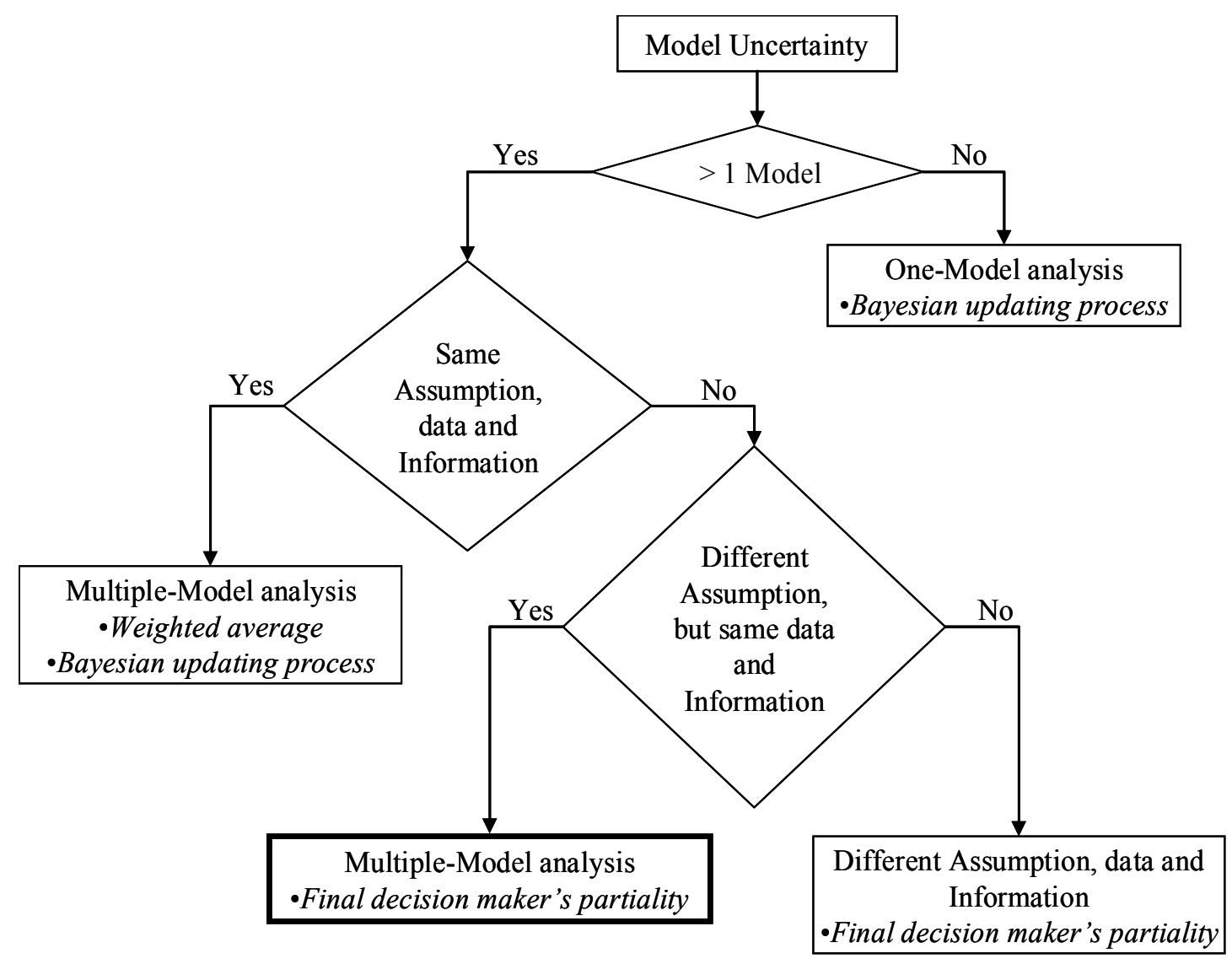

Figure 6.27 Model uncertainty analysis. 
Moreover, we identified some sources of model uncertainty applicable to damage crack growth model, which should be considered by the decision maker. These criteria are based on the work developed by Isukapalli [127]:

- Model details: Often, models are simplified for the purpose of tractability. When used to calculate the crack growth caused by fatigue, this simplification can produce conservative results, because parameters that affect the output of interests could not be taken into account. On the other hand, to include too much detail could increase the uncertainty. For example, if the manager decides to use the Forman model, the results will be considered conservative in comparison with the other two models, because the Forman model does not take into account the retardation effect.

- Model Boundaries: All models have boundaries in terms of time, space, number of chemical species, types of pathways, and so on. The selection of a boundary may be a type of simplification. Within the boundary, the model may be an accurate representation, but other overlooked phenomenon may play a role in the scenario being modeled.

In the case of crack growth, there are models whose boundary may be the intermediate region, the threshold region or the unstable rapid growth region. For example, if the manager decides to use the Walker model, the results will not be accurate, if the stress intensity factors range is close to the unstable rapid growth region. 
- Model Extrapolation: Models that are validated for one portion of input space may be completely inappropriate for making predictions in other regions of the parameter space. In the case of crack growth, it is important to evaluate if the model is able to make good predictions considering the three region of the crack growth behavior. For example, if the manager is aware that stress intensity factors range is close to the unstable rapid growth region, he/she should discard the Walker model and use the Forman and/or Closure model, which take into account this effect.

In the following chapter, the results at $1000 \Delta \mathrm{FH}$ will be discussed. In this analysis, the loads were the same as those used in the previous calculations. The PCGROW code was modified in order to sample random loads when the $F L E=j \%$ is reached. 


\section{Chapter $7 \quad$ Analysis of the results}

\subsection{Monte Carlo Simulation}

From the Monte Carlo simulation results, significant facts are worth summarizing. First, the crack growth graph shows that there are a great number of solutions where the variability in material properties should be considered. Therefore, the use of this factor will improve the damage crack growth calculation. Figure 7.1 illustrates an example of 10 iterations for a specific aircraft at $297 \%$ FLE. The variations in crack size are due to material variability, about $50 \%$ of the crack sizes at this FLE are below 0.0393 ", and $75 \%$ are below 0.0613 ".

Second, the results highlight the importance of using a probabilistic approach to analyze this kind of problems. A deterministic approach would base the prediction on the mean of the crack size, and it would not account for the fact that there is a $50 \%$

probability to have a crack size above that value. Clearly this additional information benefits the decision making process. 


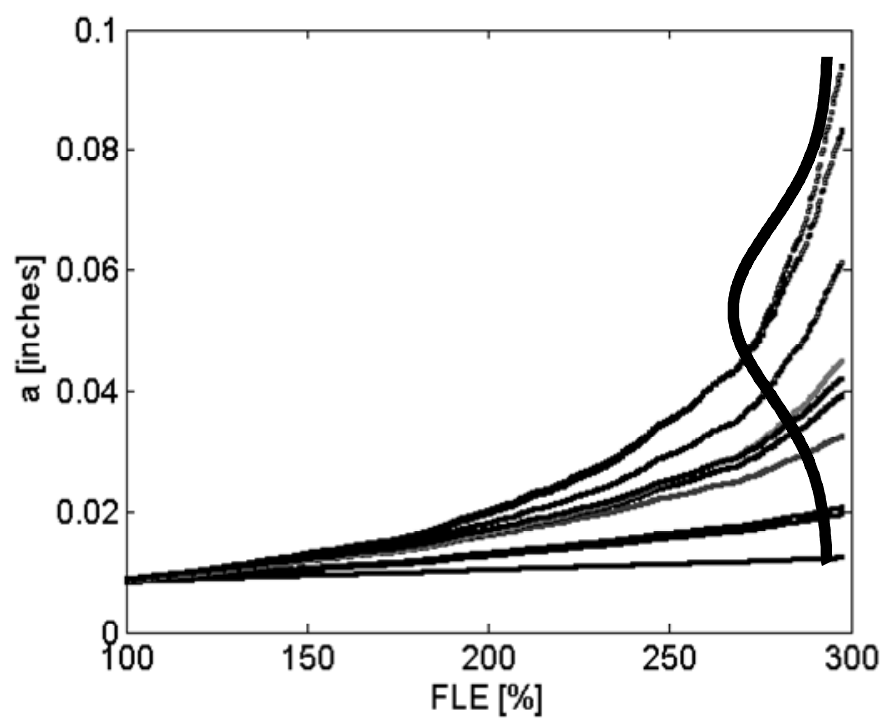

Figure 7.1 Examples of random crack growth trajectories at 297\% FLE.

\subsection{Crack Growth Model Comparisons}

Figure 7.2 illustrates the crack growth versus FLE for two aircrafts. The results show the effect of model uncertainty. The closure model is the least conservative one and reaches the critical crack size much later than the other two models. This model account for the retardation phenomenon, which realistically exits and extends the life substantially. The Forman model, which include the effect of the ratio stress $(R)$ and the instability of the crack growth when the stress-intensity factor approaches the $K_{\mathrm{C}}$ value for the material, and the Walker model, which only consider the effect of the ratio stress $(R)$ are far more conservative. This is important information for the decision maker, because it allows comparing the damage crack models determining that (in general) for all the crack size ranges, the Walker model is 
the most conservative, followed by the Forman model, and the closure model being the least conservative.
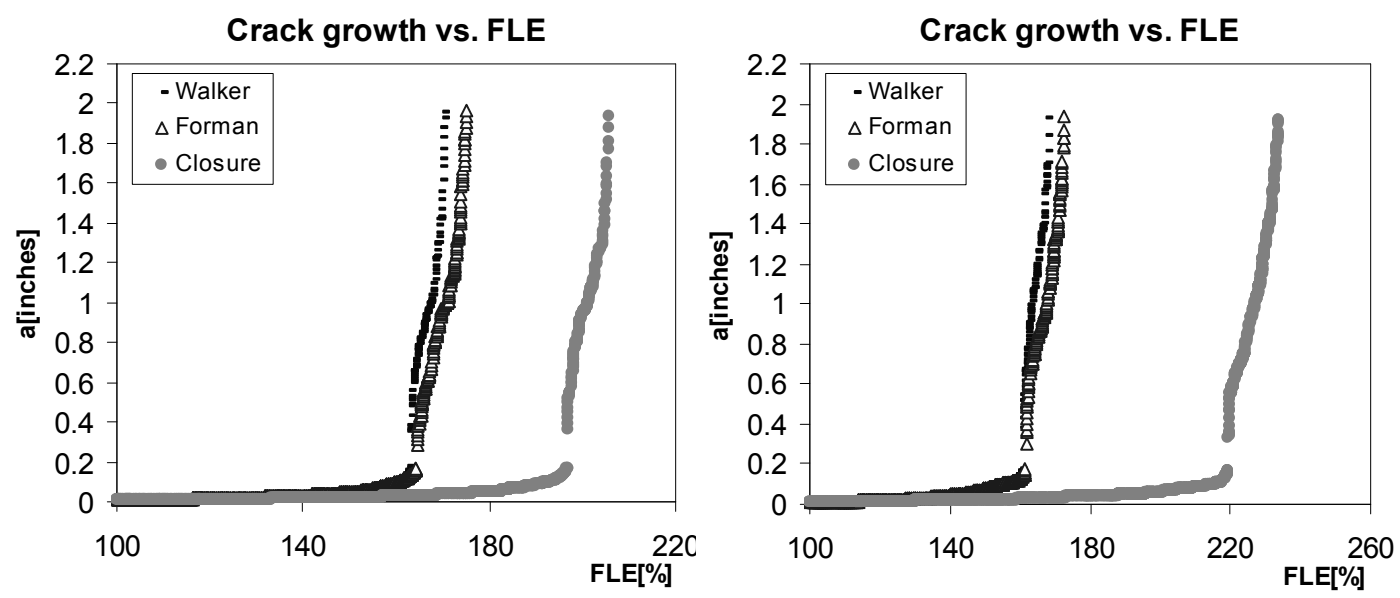

Figure 7.2 Crack growth vs. FLE.

\subsection{Selection of Lognormal Distribution}

Two goodness of fit techniques were used to determine the distribution that best represent the damage crack growth at the FLE of interest. From these results, the lognormal distribution proves to be the best fit. The normal distribution also shows good fit for some cases. In contrast, Weibull distribution does not show a good fit for the data. This supports the assumption of modeling the prior distribution of crack size as lognormal distribution in the proposed Bayesian updating calculation. Figure 7.3 shows an example of the goodness of fit analysis in a probability plot paper. This comparison graph superimposes the input data and fitted distribution on the same graph, allowing you to visually compare them as density curves [88]. In addition, this graph allows you to determine that the fitted distribution matches the input data in specific areas. For example, it is important to have a good match around the mean or in the tails. 


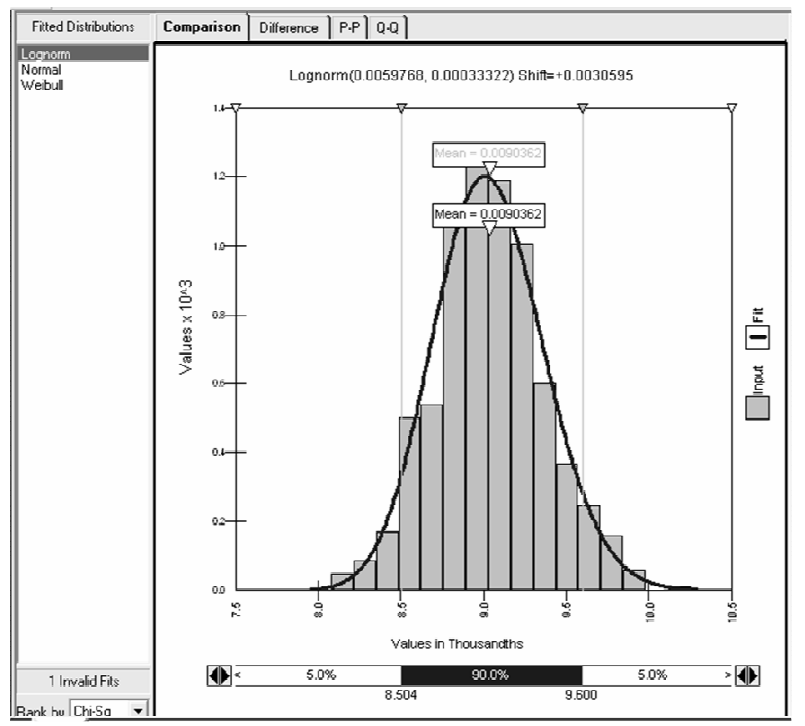

Figure 7.3 Example of lognormal fit for aircraft 4.

\subsection{Parameter Estimation}

Three methodologies were used to estimate the parameters of the IPLLognormal model. 1) GA (deterministic method), 2) MLE (a statistical method), and 3) MCMC (a probabilistic method). The results (mean values) from the three methods although valid provide the following insights. The MLE is the preferred method, because it gives very precise estimate when dealing with large data (within a 95\% confidence level). Recall that the MLE estimates can be very precise when the sample size is sufficiently large. The dependency of the GA and MCMC methods on the MLE for initial value, and their level of computational intensity requirements also support the choice for the MLE. Figure 7.4 illustrate these methods, and Figure 7.5 shows an example of the parameter estimation. 


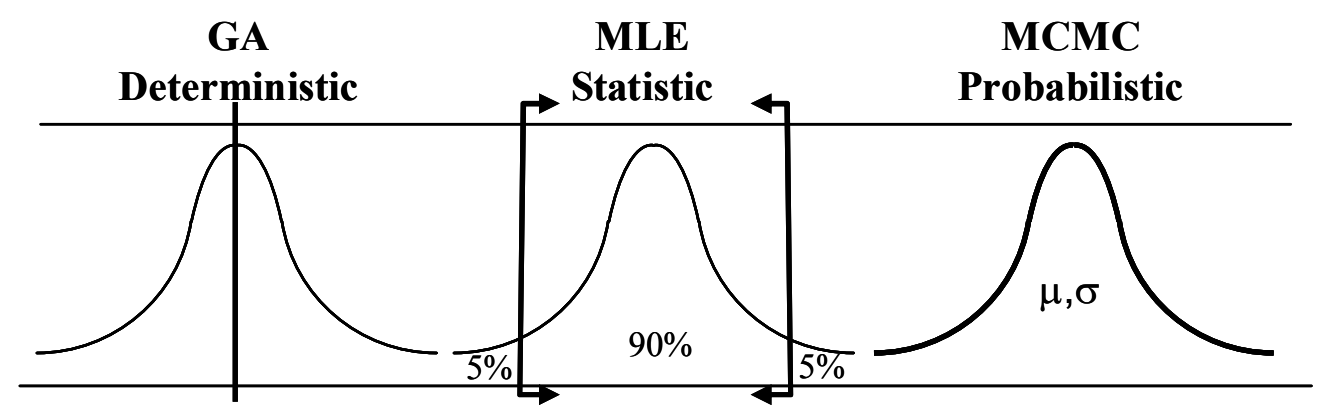

Figure 7.4 Comparison of parameter estimation methodologies.
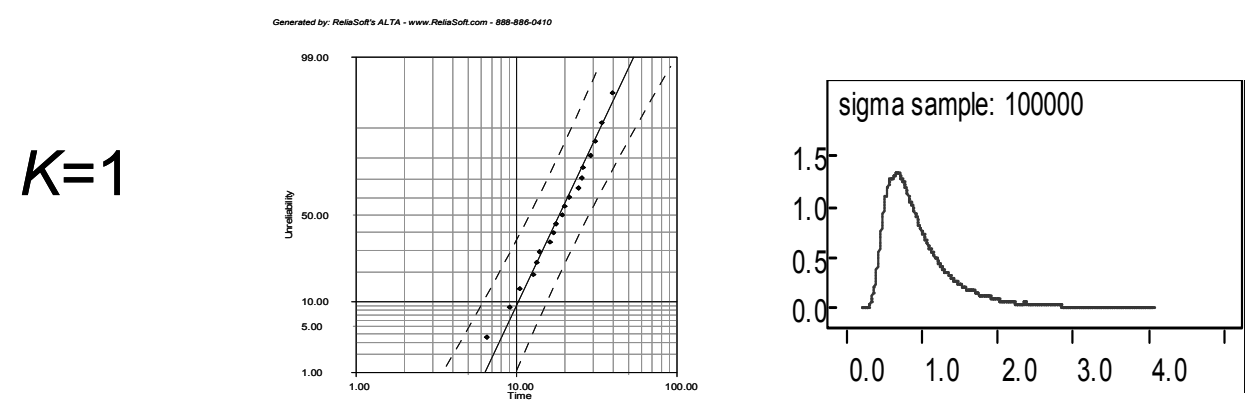

Figure 7.5 Examples of parameter estimation.

\subsection{Bayesian Analysis}

The Bayesian updating results in Chapters 5 and 6 depend on the quality and amount of evidence (inspection data) available for the analysis. Bayes' theorem measures how much new evidence would alter a belief in a hypothesis. In this study, the evidence was reported using four bin of crack sizes (extra small, small, large and extra large) and the database reports the number of cracks in each bin observed for each aircraft inspected. Figures $7.6-7.7$ illustrate that the aircrafts with larger 
number of cracks have their posterior distribution shifted to the right, which contribute more to the adjustment of the initial crack size.
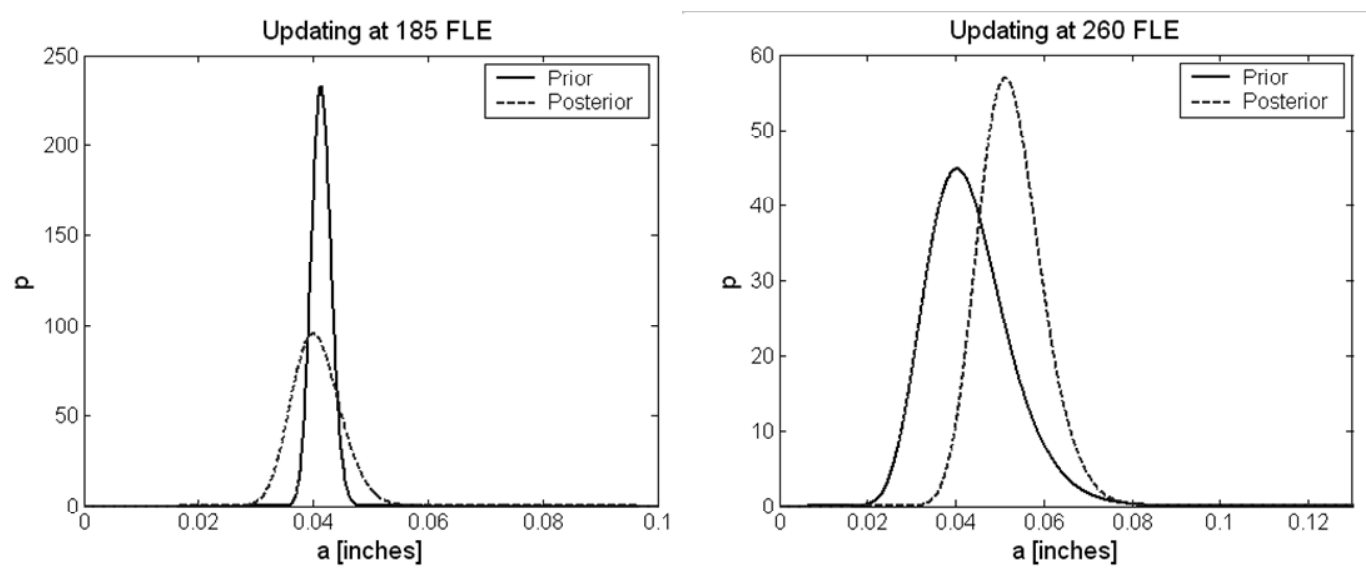

Figure 7.6 Prior and post for two aircraft evidential datasets (4 cracks size "XS") and (1 crack size "XS", 1 crack size "S".)
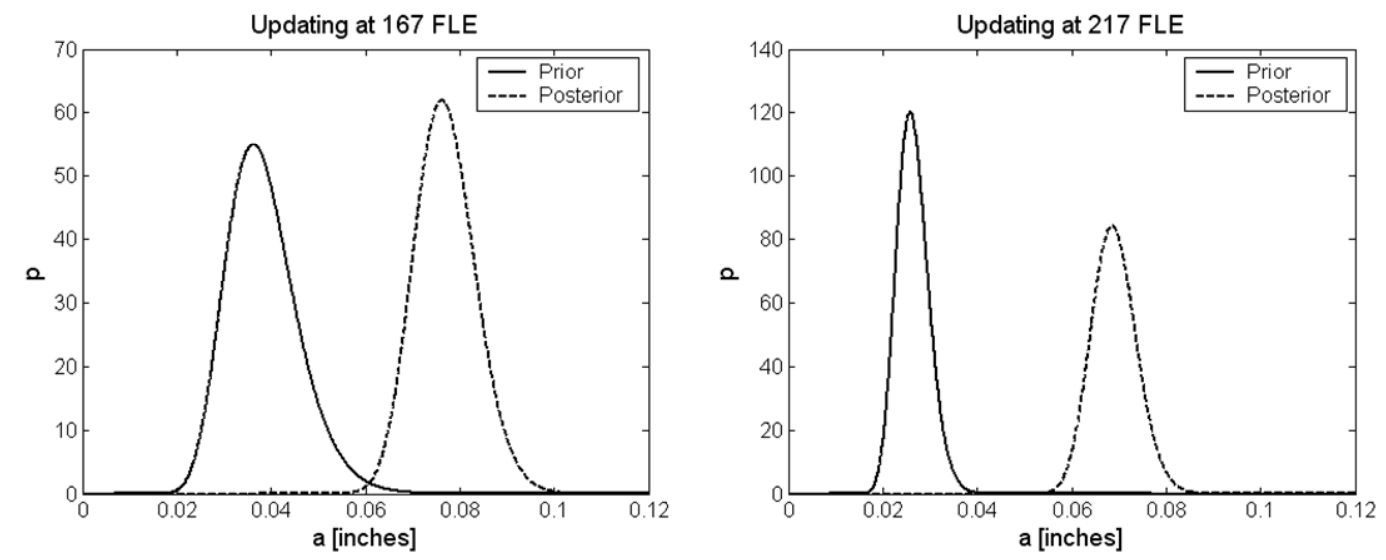

Figure 7.7 Prior and post for two aircraft evidential datasets ( 7 cracks size "S") and (5 cracks size "XS", 2 cracks size "S", 2 cracks size "L".)

The results of the posterior initial crack distribution using the conjugate approach are different from the MCMC approach. The difference is because both methods used two different likelihood functions, which are determined by the way of treating the evidences. In addition, the difference in the results is attributed to the fact 
that the posterior distributions were calculated using different procedures (See Table 7.1). Figure 7.8 illustrate the difference for two aircrafts.
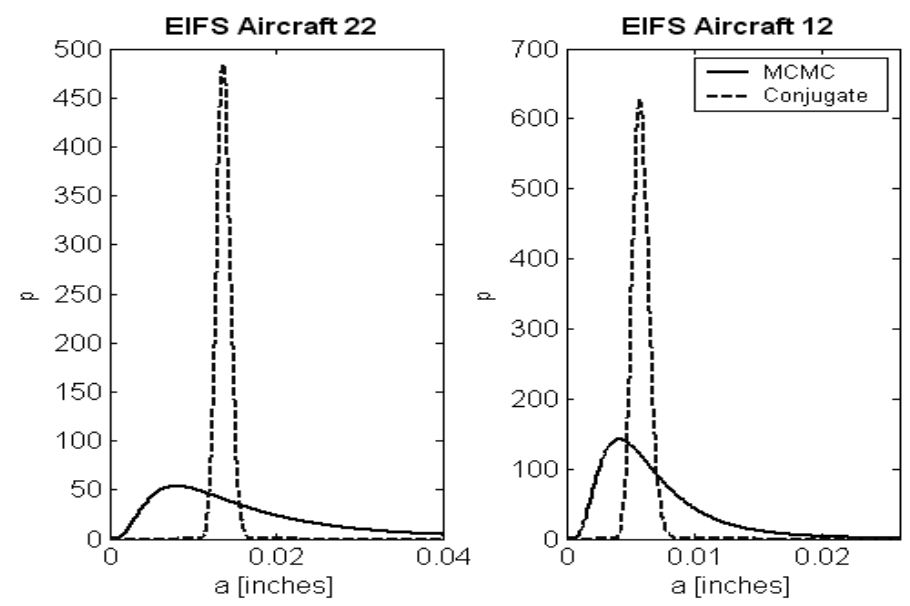

Figure 7.8 initial crack size for aircrafts 12 and 22 using conjugate and MCMC approach.

For the Conjugate approach, a set of posterior lognormal parameters is obtained for each crack growth model (Forman, Walker, Closure). They are the outcome of combining the evidence with the prior $p d f$ ' resulting from the Monte Carlo simulation (for each model) at the FLE of interest. The result from the conjugate is a lognormal distribution with its parameters. For the MCMC approach, only one set of posterior IPL-Lognormal parameters is obtained, which are used in Equation 5.12 to obtain the posterior lognormal distribution. The prior IPLlognormal parameters are originally assumed from a non-informative distribution, and they are updated directly with the evidence. Furthermore, both approaches handle the evidence in a different manner. In the conjugate approach, a multiplicative error factor is used to build the likelihood function. In the MCMC, the evidence is used 
without any modification. Table 7.1 shows the difference between the two approaches.

Table 7.1 Differences between Conjugate and MCMC approach.

\begin{tabular}{|c|c|c|}
\hline & \multicolumn{2}{|c|}{ Approach } \\
\hline & Conjugate & МСМC \\
\hline Prior & $\begin{array}{l}\text { The prior } p d f \text { is the results of the } \\
\text { IPL-Lognormal parameters } \\
\text { estimated from the Monte Carlo } \\
\text { simulation and Equation } 5.10 \text {. }\end{array}$ & $\begin{array}{l}\text { The parameters of the IPL- } \\
\text { lognormal are sampled from non- } \\
\text { informative } p d f s \text {. }\end{array}$ \\
\hline Likelihood & $\begin{array}{l}\text { A multiplicative error factor is } \\
\text { used to cover the possible crack } \\
\text { size ranges. }\end{array}$ & $\begin{array}{l}\text { The evidence is used without } \\
\text { ranges. }\end{array}$ \\
\hline Posterior & $\begin{array}{l}\text { The posterior } p d f \text { is obtained from } \\
\text { the conjugated Equations } 6.9 \text { and } \\
6.10\end{array}$ & $\begin{array}{l}\text { The posterior } p d f \text { is obtained from } \\
\text { the Equation } 5.9\end{array}$ \\
\hline
\end{tabular}

Three different initial crack size distributions at 100\% FLE were obtained, one from each crack growth model used in the calculations. These results are expected because they should reach the same evidence at the FLE where the data were recorded, even if they are based on different assumptions. Furthermore, these results confirm the conclusions of the Bell study [125] that initial distributions depend on the crack growth method used. Figure 7.9 illustrates that the closure model, which consider the retardation effect, is shifted to the right. In the same manner, the model with less variables affecting the growth, such as Walker model (only consider the ratio effect), is the one shifted to the left. 


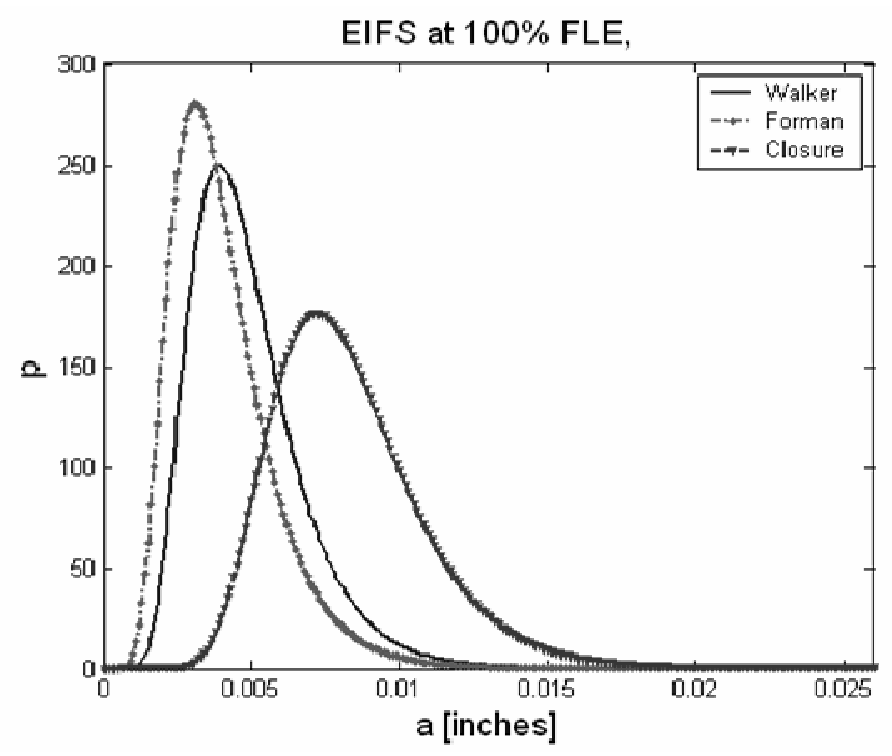

Figure 7.9 Initial crack size at $100 \%$ FLE for the three models.

The above results were obtained using the conjugate approach. A set of initial crack size distributions also were obtained using the MCMC approach. Table 7.2 shows a comparison between both approaches. The difference in the results is a consequence of the posterior distribution explained in the above paragraphs.

Table 7.2 Initial crack size Conjugate and MCMC at 100\% FLE.

\begin{tabular}{|l|c|c|c|c|}
\hline \multirow{2}{*}{ Model } & \multicolumn{2}{|c|}{ Conjugate } & \multicolumn{2}{c|}{ MCMC } \\
\cline { 2 - 5 } & $\mu_{\mathbf{L N}}$ & $\sigma_{\mathbf{L N}}$ & $\mu_{\mathbf{L N}}$ & $\sigma_{\mathbf{L N}}$ \\
\hline Walker & -5.40 & 0.38 & -5.76 & 0.69 \\
\hline Forman & -5.60 & 0.42 & -5.82 & 0.79 \\
\hline Closure & -4.84 & 0.30 & -4.80 & 0.79 \\
\hline
\end{tabular}

The same procedure to estimate the initial crack size at $100 \%$ FLE was used to estimate the parameters of the damage crack growth models. The graphs in Chapter 6 show that $m_{1}, m_{2}$, and $\gamma$ are the parameters most affected by the evidence, while the $E$ 
and $S_{y}$ do not show significant variation. Figure 7.10 shows the parameter with most variation and the parameter with the least variation.
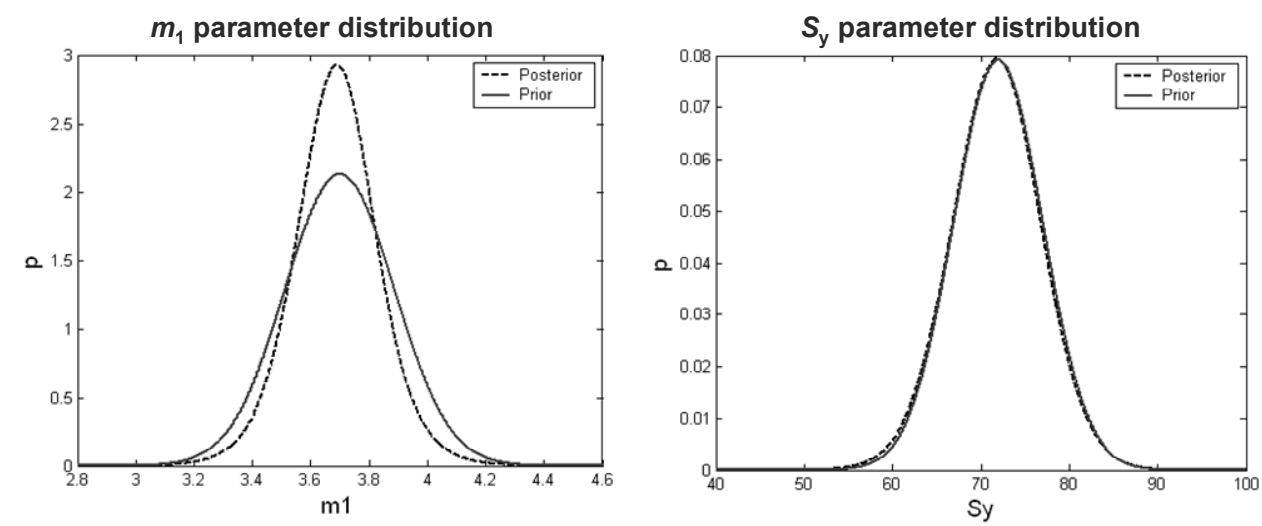

Figure 7.10 Example of posterior $p d f$ crack growth models parameter.

\subsection{Decision Making}

This research uses three different damage crack growth models to estimate the probability of having cracks of different sizes. Tables 7.3 to 7.4 show three sets of predictions at $\Delta \mathrm{H}=1000$ of additional flight hours for a given aged aircraft. This reflects the fact that there is not standard methodology used to perform fatigue life predictions for aircraft structures spectrum loading. In the majority of the cases, the Forman model yields the highest probability and the Closure model the lowest probability. These results correspond to the facts that The Forman and Walker models are more conservative than the Closure model. 
Table 7.3 Prediction results $\Delta \mathrm{FH}=1000$ - crack size XS.

\begin{tabular}{|c|c|c|c|}
\hline & Forman & Walker & Closure \\
\hline & $\operatorname{Pr}(a>$ XS $)$ & $\operatorname{Pr}(a>$ XS $)$ & $\operatorname{Pr}(a>$ XS $)$ \\
\hline 1 & $7.23 \mathrm{E}-01$ & $6.23 \mathrm{E}-01$ & $5.43 \mathrm{E}-01$ \\
\hline 2 & $9.35 \mathrm{E}-01$ & $8.43 \mathrm{E}-01$ & $8.42 \mathrm{E}-01$ \\
\hline 3 & $9.78 \mathrm{E}-01$ & $9.24 \mathrm{E}-01$ & $9.13 \mathrm{E}-01$ \\
\hline 4 & $7.38 \mathrm{E}-01$ & $6.23 \mathrm{E}-01$ & $5.76 \mathrm{E}-01$ \\
\hline 5 & $9.33 \mathrm{E}-01$ & $8.46 \mathrm{E}-01$ & $8.81 \mathrm{E}-01$ \\
\hline 6 & $9.47 \mathrm{E}-01$ & $8.75 \mathrm{E}-01$ & $8.46 \mathrm{E}-01$ \\
\hline 7 & $6.74 \mathrm{E}-01$ & $6.26 \mathrm{E}-01$ & $5.11 \mathrm{E}-01$ \\
\hline 8 & $9.34 \mathrm{E}-01$ & $8.35 \mathrm{E}-01$ & $8.58 \mathrm{E}-01$ \\
\hline 9 & $9.61 \mathrm{E}-01$ & $8.83 \mathrm{E}-01$ & $8.46 \mathrm{E}-01$ \\
\hline 10 & $9.75 \mathrm{E}-01$ & $8.95 \mathrm{E}-01$ & $8.98 \mathrm{E}-01$ \\
\hline 11 & $8.60 \mathrm{E}-01$ & $7.58 \mathrm{E}-01$ & $7.94 \mathrm{E}-01$ \\
\hline 12 & $9.33 \mathrm{E}-01$ & $8.59 \mathrm{E}-01$ & $8.45 \mathrm{E}-01$ \\
\hline 13 & $9.24 \mathrm{E}-01$ & $8.37 \mathrm{E}-01$ & $8.64 \mathrm{E}-01$ \\
\hline 14 & $9.61 \mathrm{E}-01$ & $8.65 \mathrm{E}-01$ & $8.86 \mathrm{E}-01$ \\
\hline 15 & $8.32 \mathrm{E}-01$ & $7.45 \mathrm{E}-01$ & $7.33 \mathrm{E}-01$ \\
\hline 16 & $9.24 \mathrm{E}-01$ & $8.21 \mathrm{E}-01$ & $8.48 \mathrm{E}-01$ \\
\hline 17 & $9.65 \mathrm{E}-01$ & $8.76 \mathrm{E}-01$ & $9.22 \mathrm{E}-01$ \\
\hline 18 & $5.37 \mathrm{E}-01$ & $4.96 \mathrm{E}-01$ & $3.54 \mathrm{E}-01$ \\
\hline 19 & $9.00 \mathrm{E}-02$ & $1.17 \mathrm{E}-01$ & $6.92 \mathrm{E}-03$ \\
\hline 20 & $8.30 \mathrm{E}-01$ & $7.51 \mathrm{E}-01$ & $7.14 \mathrm{E}-01$ \\
\hline 21 & $7.22 \mathrm{E}-01$ & $5.80 \mathrm{E}-01$ & $5.32 \mathrm{E}-01$ \\
\hline 22 & $6.64 \mathrm{E}-01$ & $5.67 \mathrm{E}-01$ & $4.27 \mathrm{E}-01$ \\
\hline 23 & $5.47 \mathrm{E}-01$ & $4.16 \mathrm{E}-01$ & $1.90 \mathrm{E}-01$ \\
\hline 24 & $6.85 \mathrm{E}-03$ & $1.63 \mathrm{E}-02$ & $1.12 \mathrm{E}-03$ \\
\hline 25 & $5.50 \mathrm{E}-02$ & $1.19 \mathrm{E}-01$ & $1.59 \mathrm{E}-02$ \\
\hline & & & \\
\hline
\end{tabular}


Table 7.4 Prediction results $\Delta \mathrm{FH}=1000-$ crack size S.

\begin{tabular}{|c|c|c|c|}
\hline & Forman & Walker & Closure \\
\hline & $\operatorname{Pr}(a>$ S $)$ & $\operatorname{Pr}(a>$ S $)$ & $\operatorname{Pr}(a>$ S $)$ \\
\hline 1 & $4.30 \mathrm{E}-01$ & $3.37 \mathrm{E}-01$ & $1.81 \mathrm{E}-01$ \\
\hline 2 & $7.94 \mathrm{E}-01$ & $6.18 \mathrm{E}-01$ & $5.90 \mathrm{E}-01$ \\
\hline 3 & $9.01 \mathrm{E}-01$ & $7.64 \mathrm{E}-01$ & $7.07 \mathrm{E}-01$ \\
\hline 4 & $4.23 \mathrm{E}-01$ & $3.05 \mathrm{E}-01$ & $1.92 \mathrm{E}-01$ \\
\hline 5 & $7.94 \mathrm{E}-01$ & $6.36 \mathrm{E}-01$ & $6.60 \mathrm{E}-01$ \\
\hline 6 & $8.22 \mathrm{E}-01$ & $6.79 \mathrm{E}-01$ & $5.95 \mathrm{E}-01$ \\
\hline 7 & $3.51 \mathrm{E}-01$ & $3.26 \mathrm{E}-01$ & $1.30 \mathrm{E}-01$ \\
\hline 8 & $7.90 \mathrm{E}-01$ & $6.15 \mathrm{E}-01$ & $6.09 \mathrm{E}-01$ \\
\hline 9 & $8.49 \mathrm{E}-01$ & $6.88 \mathrm{E}-01$ & $5.98 \mathrm{E}-01$ \\
\hline 10 & $8.87 \mathrm{E}-01$ & $7.06 \mathrm{E}-01$ & $6.76 \mathrm{E}-01$ \\
\hline 11 & $6.44 \mathrm{E}-01$ & $5.07 \mathrm{E}-01$ & $5.00 \mathrm{E}-01$ \\
\hline 12 & $7.81 \mathrm{E}-01$ & $6.29 \mathrm{E}-01$ & $5.73 \mathrm{E}-01$ \\
\hline 13 & $7.69 \mathrm{E}-01$ & $6.19 \mathrm{E}-01$ & $6.13 \mathrm{E}-01$ \\
\hline 14 & $8.53 \mathrm{E}-01$ & $6.60 \mathrm{E}-01$ & $6.49 \mathrm{E}-01$ \\
\hline 15 & $6.11 \mathrm{E}-01$ & $4.82 \mathrm{E}-01$ & $4.31 \mathrm{E}-01$ \\
\hline 16 & $7.65 \mathrm{E}-01$ & $5.89 \mathrm{E}-01$ & $5.73 \mathrm{E}-01$ \\
\hline 17 & $8.58 \mathrm{E}-01$ & $6.79 \mathrm{E}-01$ & $7.11 \mathrm{E}-01$ \\
\hline 18 & $2.31 \mathrm{E}-01$ & $2.16 \mathrm{E}-01$ & $6.44 \mathrm{E}-02$ \\
\hline 19 & $6.60 \mathrm{E}-03$ & $1.30 \mathrm{E}-02$ & $2.40 \mathrm{E}-06$ \\
\hline 20 & $5.82 \mathrm{E}-01$ & $4.76 \mathrm{E}-01$ & $3.88 \mathrm{E}-01$ \\
\hline 21 & $4.15 \mathrm{E}-01$ & $2.84 \mathrm{E}-01$ & $1.59 \mathrm{E}-01$ \\
\hline 22 & $3.73 \mathrm{E}-01$ & $2.84 \mathrm{E}-01$ & $9.16 \mathrm{E}-02$ \\
\hline 23 & $2.32 \mathrm{E}-01$ & $1.47 \mathrm{E}-01$ & $8.78 \mathrm{E}-03$ \\
\hline 24 & $4.41 \mathrm{E}-05$ & $2.18 \mathrm{E}-04$ & $4.46 \mathrm{E}-08$ \\
\hline 25 & $2.34 \mathrm{E}-03$ & $1.12 \mathrm{E}-02$ & $1.74 \mathrm{E}-05$ \\
\hline
\end{tabular}


Table 7.5 Prediction results $\Delta \mathrm{FH}=1000-$ crack size L.

\begin{tabular}{|c|c|c|c|}
\hline & Forman & Walker & Closure \\
\hline & $\operatorname{Pr}(a>\mathrm{L})$ & $\operatorname{Pr}(a>\mathrm{L})$ & $\operatorname{Pr}(a>\mathrm{L})$ \\
\hline 1 & $2.18 \mathrm{E}-01$ & $1.60 \mathrm{E}-01$ & $4.34 \mathrm{E}-02$ \\
\hline 2 & $6.08 \mathrm{E}-01$ & $4.00 \mathrm{E}-01$ & $3.51 \mathrm{E}-01$ \\
\hline 3 & $7.65 \mathrm{E}-01$ & $5.65 \mathrm{E}-01$ & $4.62 \mathrm{E}-01$ \\
\hline 4 & $1.99 \mathrm{E}-01$ & $1.24 \mathrm{E}-01$ & $4.40 \mathrm{E}-02$ \\
\hline 5 & $6.13 \mathrm{E}-01$ & $4.29 \mathrm{E}-01$ & $4.25 \mathrm{E}-01$ \\
\hline 6 & $6.48 \mathrm{E}-01$ & $4.70 \mathrm{E}-01$ & $3.56 \mathrm{E}-01$ \\
\hline 7 & $1.50 \mathrm{E}-01$ & $1.46 \mathrm{E}-01$ & $2.13 \mathrm{E}-02$ \\
\hline 8 & $6.02 \mathrm{E}-01$ & $4.04 \mathrm{E}-01$ & $3.66 \mathrm{E}-01$ \\
\hline 9 & $6.78 \mathrm{E}-01$ & $4.77 \mathrm{E}-01$ & $3.60 \mathrm{E}-01$ \\
\hline 10 & $7.35 \mathrm{E}-01$ & $4.93 \mathrm{E}-01$ & $4.29 \mathrm{E}-01$ \\
\hline 11 & $4.26 \mathrm{E}-01$ & $3.02 \mathrm{E}-01$ & $2.60 \mathrm{E}-01$ \\
\hline 12 & $5.84 \mathrm{E}-01$ & $4.00 \mathrm{E}-01$ & $3.20 \mathrm{E}-01$ \\
\hline 13 & $5.75 \mathrm{E}-01$ & $4.08 \mathrm{E}-01$ & $3.64 \mathrm{E}-01$ \\
\hline 14 & $6.87 \mathrm{E}-01$ & $4.48 \mathrm{E}-01$ & $3.97 \mathrm{E}-01$ \\
\hline 15 & $4.01 \mathrm{E}-01$ & $2.74 \mathrm{E}-01$ & $2.12 \mathrm{E}-01$ \\
\hline 16 & $5.66 \mathrm{E}-01$ & $3.75 \mathrm{E}-01$ & $3.17 \mathrm{E}-01$ \\
\hline 17 & $6.88 \mathrm{E}-01$ & $4.68 \mathrm{E}-01$ & $4.52 \mathrm{E}-01$ \\
\hline 18 & $8.32 \mathrm{E}-02$ & $8.19 \mathrm{E}-02$ & $7.84 \mathrm{E}-03$ \\
\hline 19 & $3.75 \mathrm{E}-04$ & $1.20 \mathrm{E}-03$ & $2.34 \mathrm{E}-10$ \\
\hline 20 & $3.54 \mathrm{E}-01$ & $2.61 \mathrm{E}-01$ & $1.71 \mathrm{E}-01$ \\
\hline 21 & $1.99 \mathrm{E}-01$ & $1.20 \mathrm{E}-01$ & $3.27 \mathrm{E}-02$ \\
\hline 22 & $1.81 \mathrm{E}-01$ & $1.25 \mathrm{E}-01$ & $1.29 \mathrm{E}-02$ \\
\hline 23 & $8.11 \mathrm{E}-02$ & $4.43 \mathrm{E}-02$ & $1.94 \mathrm{E}-04$ \\
\hline 24 & $2.06 \mathrm{E}-07$ & $2.13 \mathrm{E}-06$ & $4.53 \mathrm{E}-13$ \\
\hline 25 & $7.42 \mathrm{E}-05$ & $8.12 \mathrm{E}-04$ & $5.89 \mathrm{E}-09$ \\
\hline
\end{tabular}


Table 7.6 Prediction results $\Delta \mathrm{FH}=1000$ - crack size XL.

\begin{tabular}{|c|c|c|c|}
\hline & Forman & Walker & Closure \\
\hline & $\operatorname{Pr}(a>$ XL $)$ & $\operatorname{Pr}(a>$ XL $)$ & $\operatorname{Pr}(a>$ XL $)$ \\
\hline 1 & $7.18 \mathrm{E}-02$ & $4.94 \mathrm{E}-02$ & $4.39 \mathrm{E}-03$ \\
\hline 2 & $3.65 \mathrm{E}-01$ & $1.89 \mathrm{E}-01$ & $1.42 \mathrm{E}-01$ \\
\hline 3 & $5.33 \mathrm{E}-01$ & $3.19 \mathrm{E}-01$ & $2.06 \mathrm{E}-01$ \\
\hline 4 & $5.68 \mathrm{E}-02$ & $2.95 \mathrm{E}-02$ & $3.98 \mathrm{E}-03$ \\
\hline 5 & $3.76 \mathrm{E}-01$ & $2.18 \mathrm{E}-01$ & $1.91 \mathrm{E}-01$ \\
\hline 6 & $4.07 \mathrm{E}-01$ & $2.46 \mathrm{E}-01$ & $1.44 \mathrm{E}-01$ \\
\hline 7 & $3.77 \mathrm{E}-02$ & $4.09 \mathrm{E}-02$ & $1.13 \mathrm{E}-03$ \\
\hline 8 & $3.58 \mathrm{E}-01$ & $1.97 \mathrm{E}-01$ & $1.47 \mathrm{E}-01$ \\
\hline 9 & $4.27 \mathrm{E}-01$ & $2.48 \mathrm{E}-01$ & $1.48 \mathrm{E}-01$ \\
\hline 10 & $4.87 \mathrm{E}-01$ & $2.56 \mathrm{E}-01$ & $1.84 \mathrm{E}-01$ \\
\hline 11 & $2.06 \mathrm{E}-01$ & $1.29 \mathrm{E}-01$ & $8.50 \mathrm{E}-02$ \\
\hline 12 & $3.34 \mathrm{E}-01$ & $1.79 \mathrm{E}-01$ & $1.13 \mathrm{E}-01$ \\
\hline 13 & $3.33 \mathrm{E}-01$ & $2.01 \mathrm{E}-01$ & $1.42 \mathrm{E}-01$ \\
\hline 14 & $4.41 \mathrm{E}-01$ & $2.27 \mathrm{E}-01$ & $1.61 \mathrm{E}-01$ \\
\hline 15 & $1.96 \mathrm{E}-01$ & $1.09 \mathrm{E}-01$ & $6.60 \mathrm{E}-02$ \\
\hline 16 & $3.21 \mathrm{E}-01$ & $1.74 \mathrm{E}-01$ & $1.10 \mathrm{E}-01$ \\
\hline 17 & $4.32 \mathrm{E}-01$ & $2.43 \mathrm{E}-01$ & $1.87 \mathrm{E}-01$ \\
\hline 18 & $1.69 \mathrm{E}-02$ & $1.87 \mathrm{E}-02$ & $2.97 \mathrm{E}-04$ \\
\hline 19 & $5.85 \mathrm{E}-06$ & $3.82 \mathrm{E}-05$ & $2.22 \mathrm{E}-16$ \\
\hline 20 & $1.50 \mathrm{E}-01$ & $9.73 \mathrm{E}-02$ & $4.40 \mathrm{E}-02$ \\
\hline 21 & $5.92 \mathrm{E}-02$ & $3.11 \mathrm{E}-02$ & $2.53 \mathrm{E}-03$ \\
\hline 22 & $5.74 \mathrm{E}-02$ & $3.55 \mathrm{E}-02$ & $5.73 \mathrm{E}-04$ \\
\hline 23 & $1.56 \mathrm{E}-02$ & $7.22 \mathrm{E}-03$ & $5.29 \mathrm{E}-07$ \\
\hline 24 & $1.01 \mathrm{E}-10$ & $2.82 \mathrm{E}-09$ & $6.25 \mathrm{E}-16$ \\
\hline 25 & $5.05 \mathrm{E}-07$ & $1.76 \mathrm{E}-05$ & $3.80 \mathrm{E}-14$ \\
\hline
\end{tabular}

Figures 7.11 to 7.13 show the results for each aircraft using the three models with their uncertainties. As was defined in Chapter 6, this is a multiple model uncertainty case where it requires management's decision as to which of the model output would be used. 

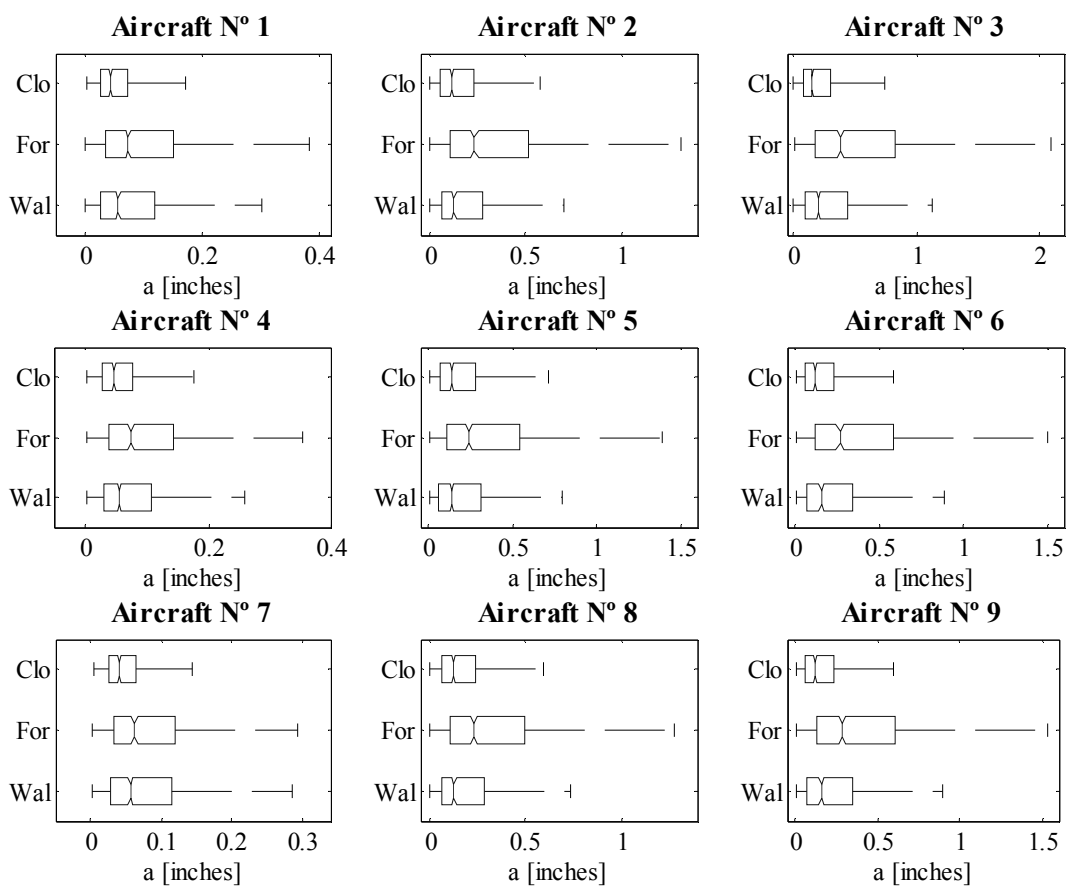

Figure 7.11 Box plot of the three models at $\Delta \mathrm{FH}=1000$ - Aircrafts 1 to 9 .
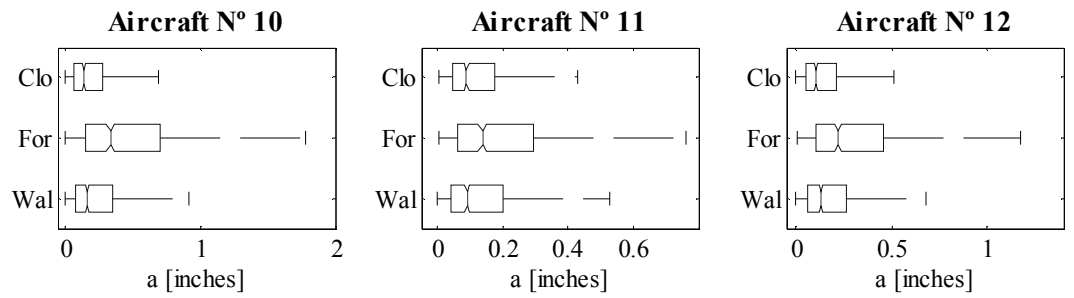

Aircraft $N^{\circ} 13$

Aircraft $N^{\circ} 14$
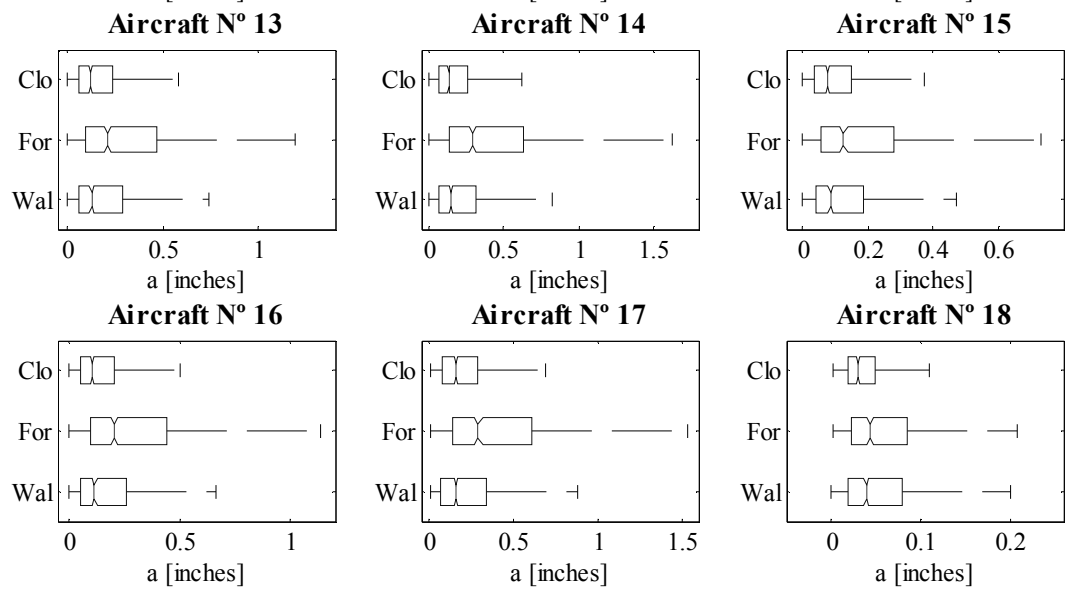

Figure 7.12 Box plot of the three models at $\Delta \mathrm{FH}=1000$ - Aircrafts 10 to 18 . 

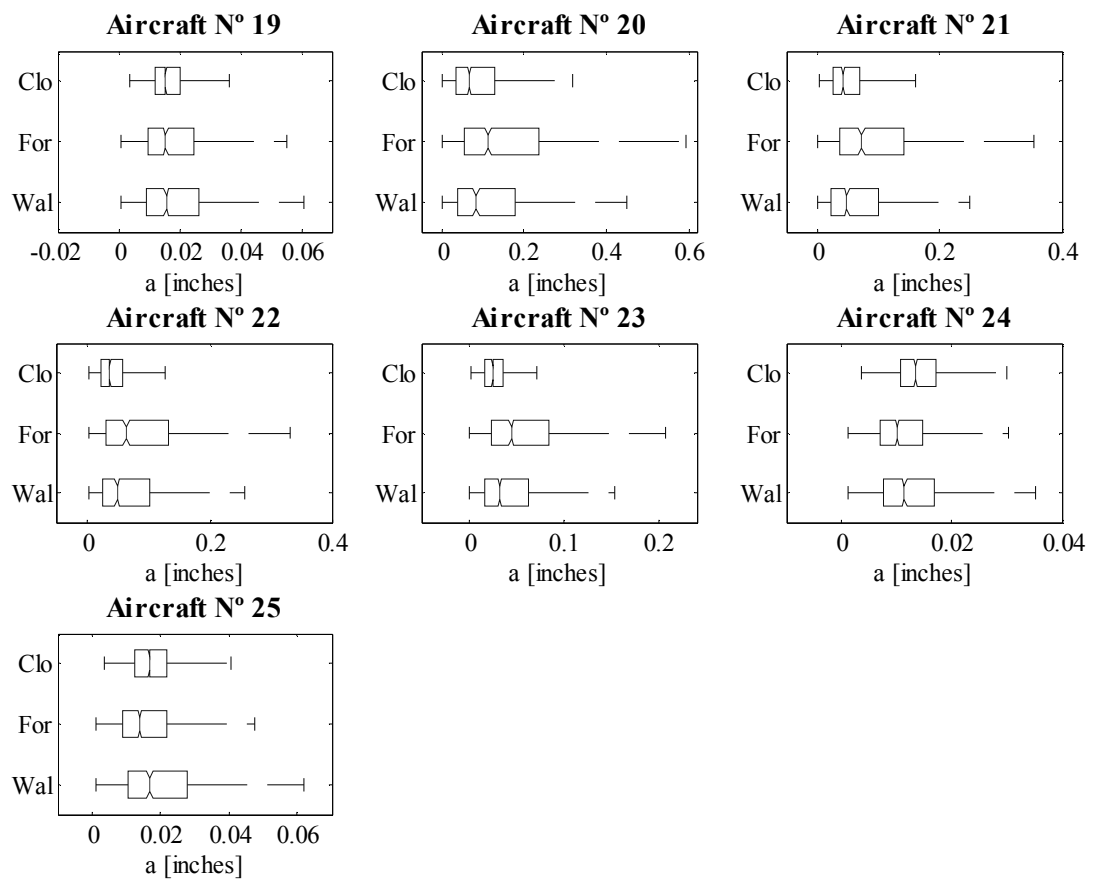

Figure 7.13 Box plot of the three models at $\Delta \mathrm{FH}=1000$ - Aircrafts 19 to 25 .

Taking into account the above statements, the results will be discussed from the point of view of risk. Uncertainty and risk are closely associated with one another, but not are identical. In this research, we identify two different decision makers in the presence of risk: one who is comfortable with risk-taking and another who is risk-averse. Figure 7.14 shows an example of the different alternatives for decision making in aircraft aging. The probability values are only for illustration of the methodology. 


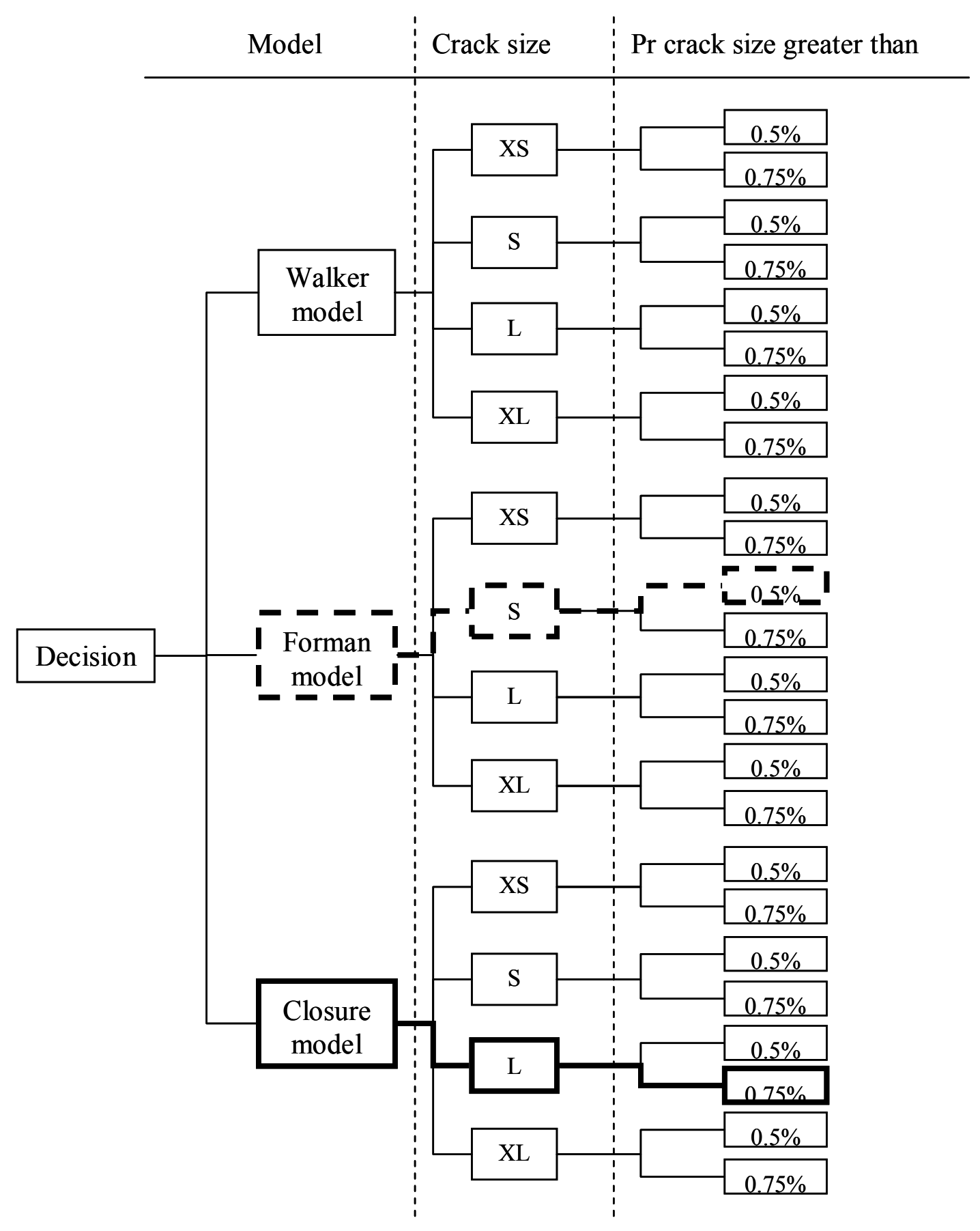

Figure 7.14 Alternatives for decision making.

According to this, a risk taker may define a probability of crack size larger than $50 \%$, and a crack growth model non-conservative in order to extend the inspection frequency, and reduce cost because of the inspection. 
For example, a risk taker would define a crack size large, "L", a probability of crack size $\mathrm{L}$ greater than $75 \%$ to perform maintenance and the crack closure model to represent the damage crack growth phenomenon. The highlighted path in Figure 7.14 shows this alternative. According to these factors, none of the aircrafts would be scheduled for maintenance at the $1000 \Delta \mathrm{FH}$.

On the other hand, a risk-averse decision maker may define a probability of crack size average, "S", greater than $50 \%$, and a conservative model, such as Forman. The dotted path in Figure 7.14 shows this alternative. In this case, 15 out of the 25 aircrafts would be scheduled for maintenance at the $1000 \Delta \mathrm{FH}$. If it is not feasible to performance maintenance over the 15 aircrafts, the decision maker could use the Box plot graphs in order to reduce the number. In this case, from the graphs, it is possible to reduce the number from 15 to 7 . Table 7.7 and 7.8 show the maintenance schedule after $1000 \mathrm{FHR}$, according to this decision maker.

Table 7.7 Aircrafts to maintenance.

\begin{tabular}{|c|c|c|c|c|c|c|c|c|c|}
\hline \multicolumn{1}{|c|}{ Aircrafts that require maintenance after 1000 FHR } \\
\hline 2 & 3 & 5 & 6 & 8 & 9 & 10 & 11 & 13 & 14 \\
\hline 15 & 16 & 17 & 20 & & & & & & \\
\hline
\end{tabular}

Table 7.8 Aircrafts to maintenance after the box plot analysis.

\begin{tabular}{|c|c|c|c|c|c|c|}
\hline \multicolumn{6}{|c|}{ Aircrafts that require maintenance after $1000 \mathrm{FHR}$} \\
\hline 3 & 5 & 6 & 9 & 10 & 14 & 17 \\
\hline
\end{tabular}

The analysis carried out in this research was developed over 25 aircrafts, however the results will be used to make probability statements on the total population of the fleet. In fact, the results obtained will be the initial assessment for decision making over a total population about 200 aircrafts. This is a continuous 
improving process; therefore data from more aircrafts has been analyzed to be included in the analysis. This new data will contribute to reduce the uncertainties, because the Bayesian updating will improve the calculation of the initial crack size and parameters, which it will help to discover the "unknown unknown" in a timely manner. 


\section{Chapter 8 Conclusions \& Recommendations}

This dissertation describes a probabilistic approach for determining crack sizes in aging aircrafts. The use of the proposed probabilistic techniques in engineering and economic analyses related to cost reduction, risk management, and cost-effective decision management is increasing. Purely deterministic approaches, although simple to use, do not consider variability and uncertainty inherent in any real-life data. As a consequence, it often results in overly conservative estimates. It is thus important to introduce the concept of variability and uncertainty in the analysis, since it usually offers a realistic representation of the process been modeled accompanied by the corresponding uncertainty bounds.

From a deterministic perspective, there is not a universal model that represents the fatigue crack growth phenomenon, particularly in aging aircrafts. Fatigue induced crack growth predictions remains an empirical science at present. Therefore, a probabilistic analysis of the different factors involved in the calculation was performed in this research in order to address these uncertainties.

There are three deterministic models that can be used for estimating crack growth: the Closure model, the Forman model, and the Walker model. The selection of any model will depend on the specific application. In general, the Forman and Walker models provide conservative estimates when compared to the Closure model.

The damage crack growth estimation and uncertainty propagation from the input variables to the fatigue life is carried out by a simple Monte Carlo simulation. The results obtained show that the methodology developed in this research is an effective approach for assessing structural health of aging aircrafts. The Bayesian 
process used to update the evidence of the crack size takes into account the inspection results and reduces the uncertainty of the initial crack size. Furthermore, consideration of the scatter in the material properties brings all of the variability into consideration and makes the estimations more objective.

The initial crack size distribution depends on the crack growth model. Therefore, it is very important to use the initial crack size that corresponds to the damage crack growth model used in the calculations.

For more accurate results, it is strongly recommended that the crack sizes be reported without binning them, and to avoid errors due to "smoothing" of the population initial crack size distribution, more aircrafts (perhaps 40 or more) be inspected and reported. Further, it is recommended to analyze around 40 aircrafts to further reduce uncertainties.

The model parameters depend on a particular application, therefore it is recommended to devise a continuous updating when new evidence is available. The parameters $m_{1}, m_{2}$, and $\gamma$ are the parameters more affected by the evidence, while the $E$ and $S_{y}$ do not show significant variation.

The parameter estimation using the Maximum Likelihood Estimation method was shown to be very reliable, however the Markov Chain Monte Carlo method allows for estimation of the distribution of the parameters, which is useful for uncertainty analysis.

The material properties play an important role in the estimation of the damage crack growth, however the results obtained show that the mean values reported in the literature can be used with a great level of confidence. 
The results obtained through the lognormal conjugate updating are satisfactory, however further analysis should be done to automate the MCMC approach, and to reduce the uncertainty about the way the data are treated. One approach would be to develop the likelihood function for each model; however, it is a complex calculation due to the number of parameters involved in the formulas.

The life estimation should be a tradeoff between accuracy and uncertainty propagation due to the number of parameters involved in the model. Some researchers to minimize risk could add complexity to realistically reflect the damage crack growth phenomenon such as the retardation effect. However, complexity means more parameters to estimate, for which more assumptions needs to be done.

To deal with a single model it is recommended to calculate the crack growth using the Closure model. Although this model is the least conservative of the models analyzed in this research, its results have been shown to be more realistic, because it considers the retardation effect, which is a phenomena present in the crack growth behavior.

The risk in building a very simple model is that it may lead to an underestimate of the risk (because all the elements may not be modeled) or to an inefficient use of the available information. The question of the appropriate level of complexity (i.e., the trade-off between precision and uncertainty) depends on the manager's decision as to which of the model outputs he/she prefers to use.

The initial crack length distribution representative of the fleet is a multimodal $p d f$ obtained from the linear arithmetic approach. In this approach, equal weights are assigned to the mechanism due to fatigue and the mechanism due to fatigue plus environmental corrosion, which gives rise to a heavy tail of the fitting lognormal 
distribution. A new approach to derive an averaged crack length for the fleet based on the Bayesian averaging would improve the calculation of this distribution.

Further analysis is necessary in order to safely extend the service life of aging aircrafts. Such studies should consider Wide Fatigue Damage (WFD), which is characterized by the simultaneous presence of cracks at multiple structural points. The two sources of WFD are multiple-site damage (MSD), characterized by the simultaneous presence of fatigue cracks in the same structural element; and multiple element damage (MED), characterized by the simultaneous presence of fatigue cracks in similar adjacent structural elements. 


\section{Appendix A PCGROW code for crack growth -closure}

\section{model example calculation.}

This is the complete MatLab code for crack growth calculation example. It is the same structure for Walker and Forman models in chapter 4 . The difference is the $\Delta K$ and $d a / d N$ equations.

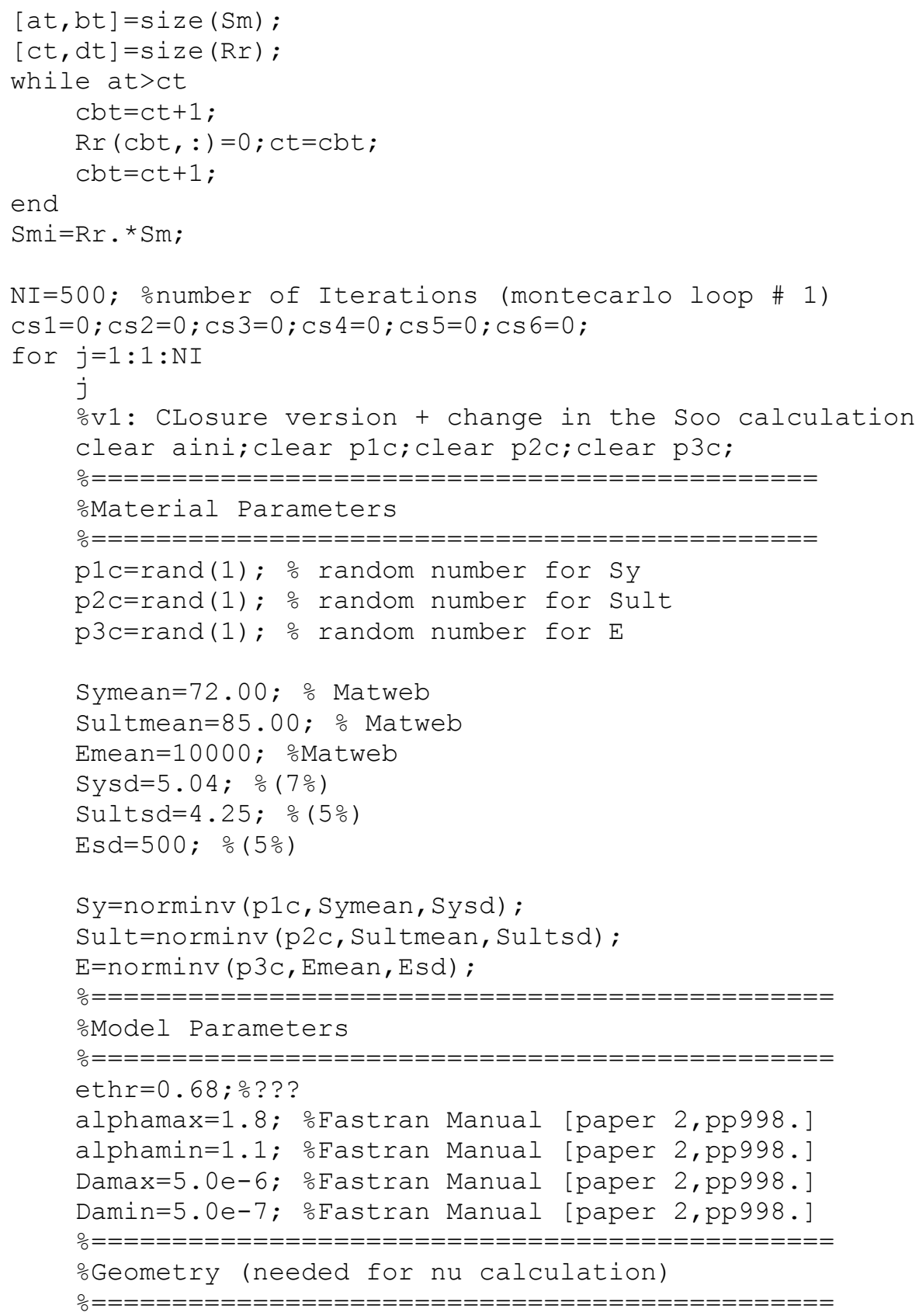




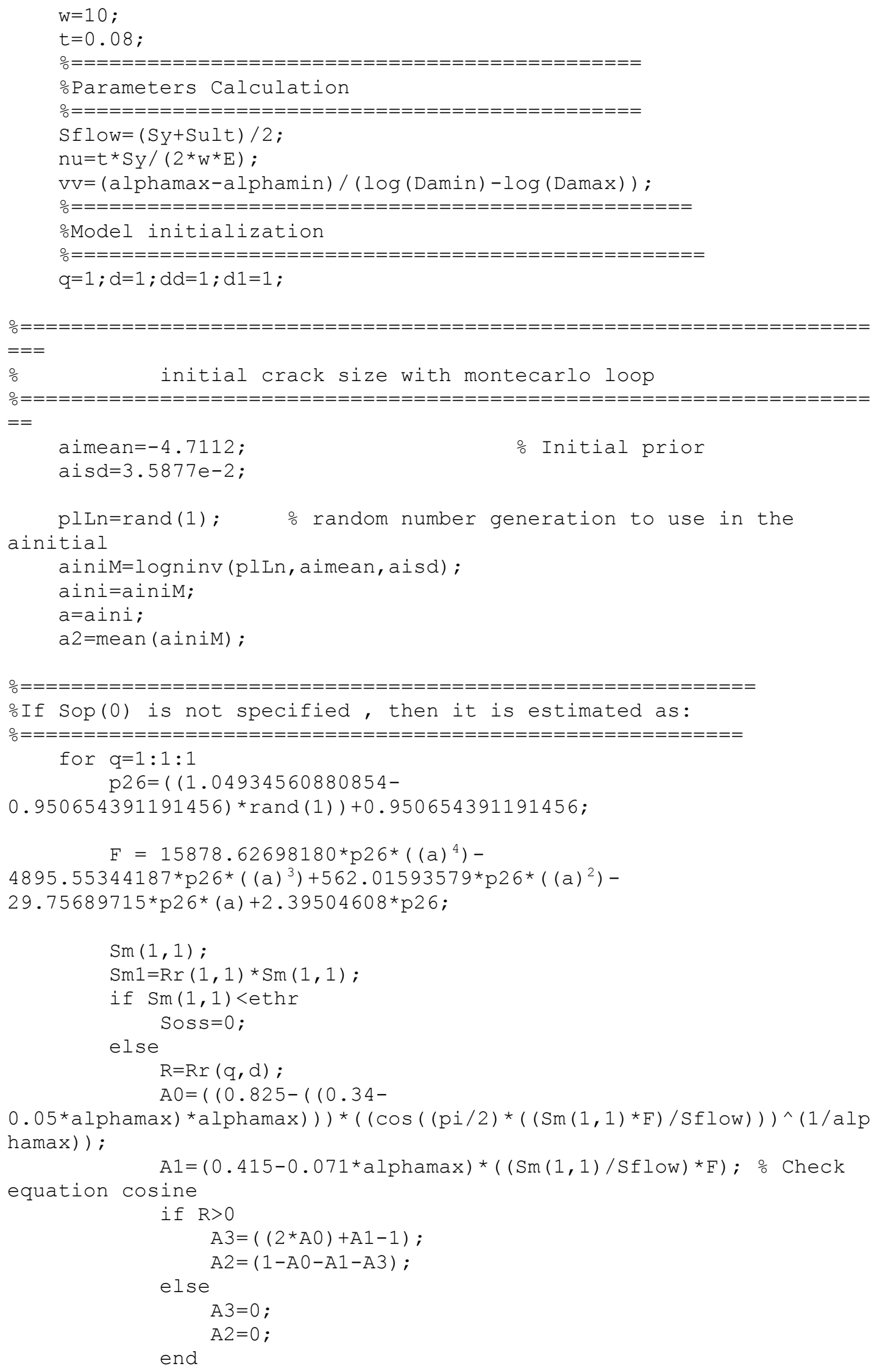




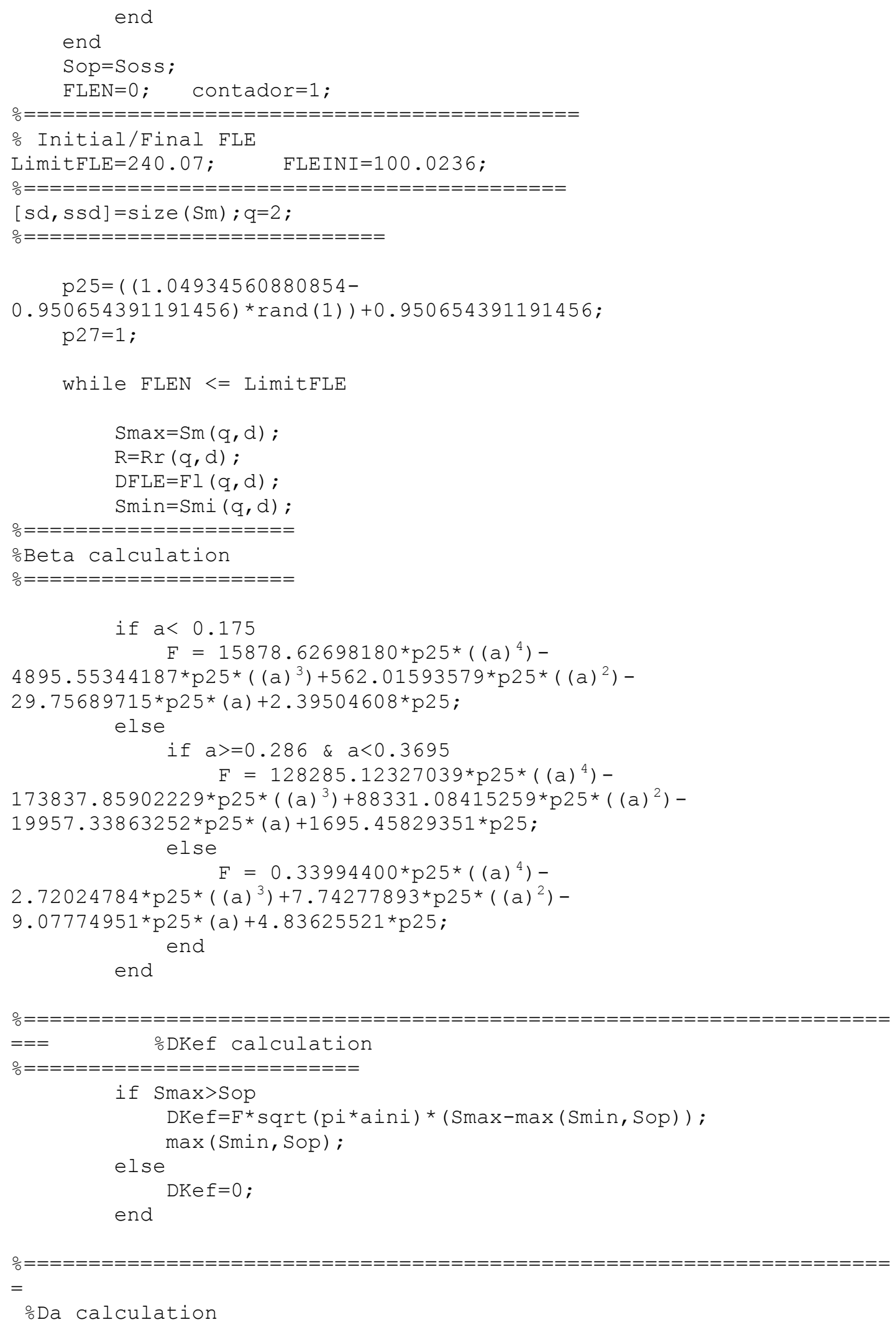




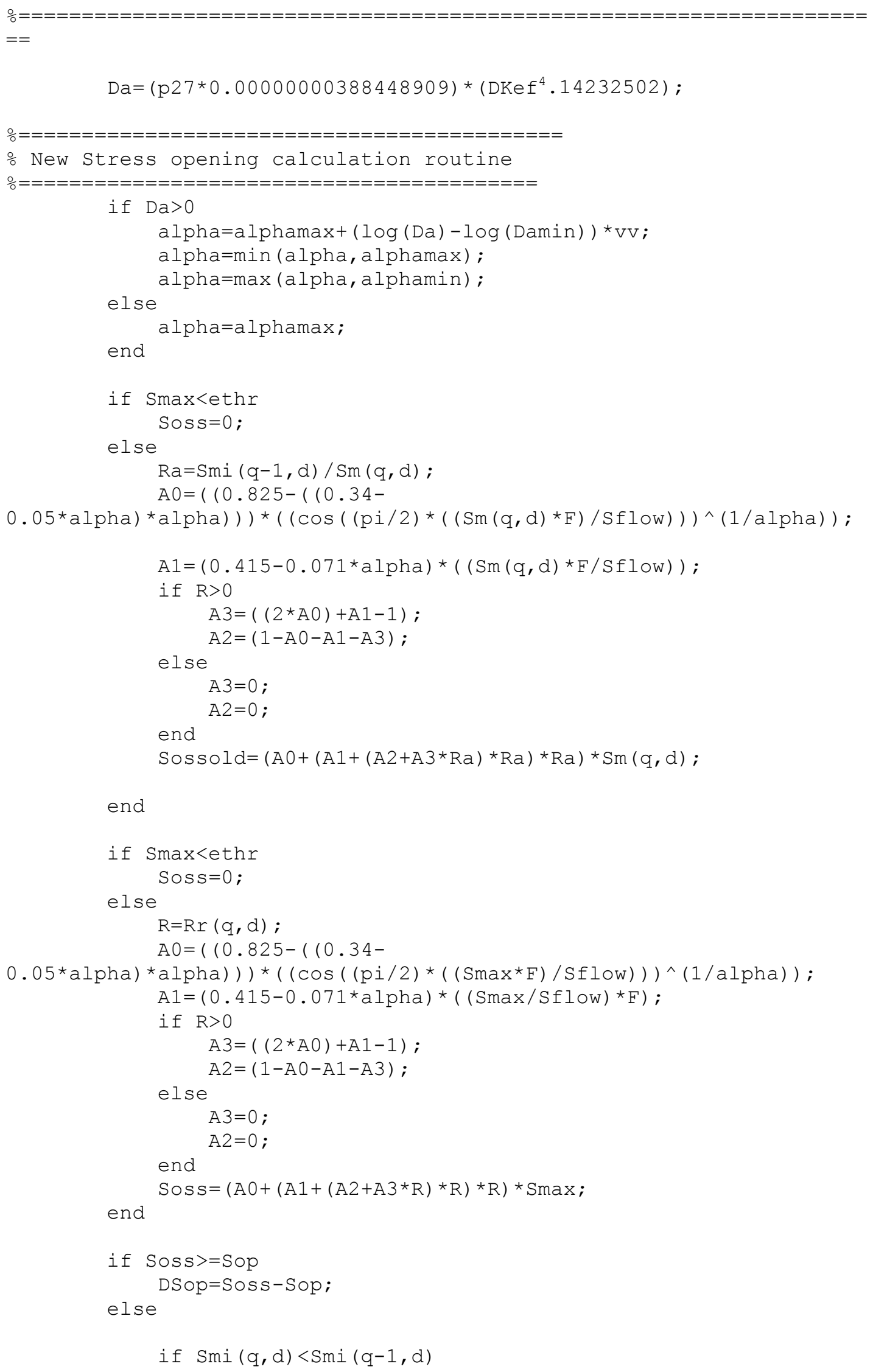




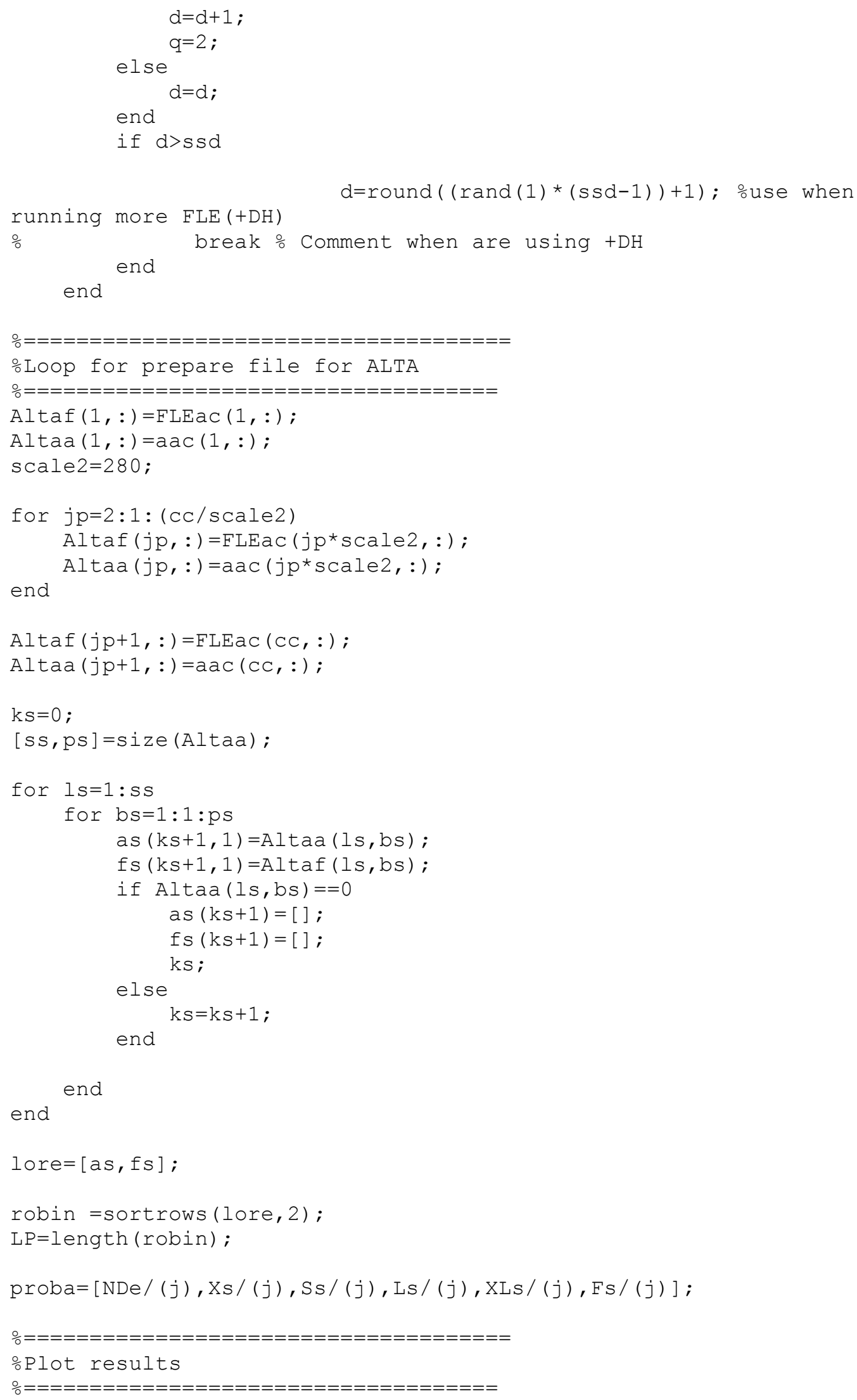




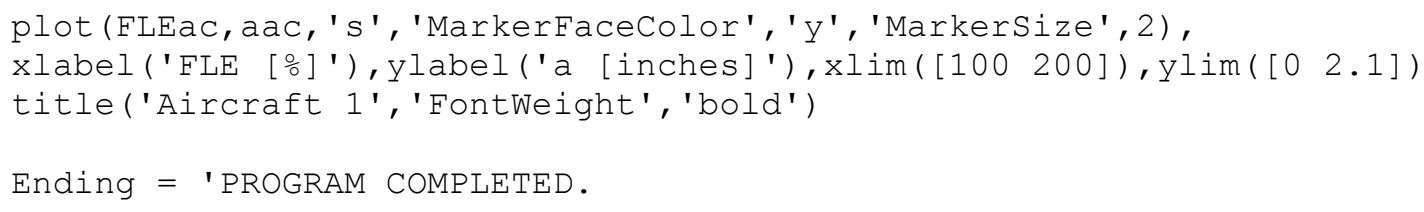




\section{Appendix B WinBUGS code for parameter estimation}

\section{example calculation.}

The WINBUGS model specification is as follows,

- Initial values for $\mathrm{K}, \mathrm{n}$ and $\sigma$ :

list $($ kapa $1=10$, neta $=3$, sigma $=0.1)$

- IPL-Lognormal model:

model;

\{

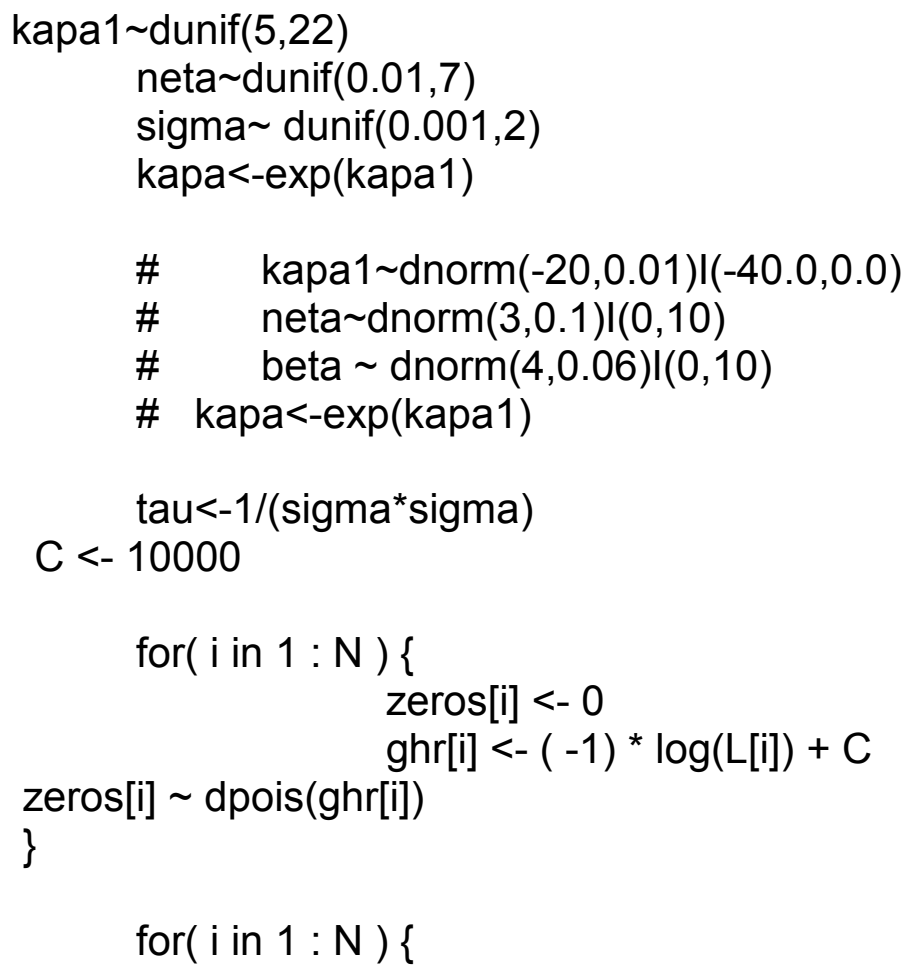

- Data ( $a$ versus $F L E)$

list $(\mathrm{tf}=$ structure $($. Data $=\mathrm{c}(0.005014,1,0.009995,0.005024,1,0.009986,0.005034,1,0.00998$ $1,0.005044,1,0.009968,0.005046,1,0.009965,0.005048,1,0.00996,0.005062,1,0.009949,0.00$ $5062,1,0.009947,0.005063,1,0.009945,0.005075,1,0.00994,0.005093,1,0.009924,0.005101$, $1,0.00992,0.005109,1,0.009915,0.005123,1,0.009902,0.005135,1,0.009896,0.005154,1,0.00$ $9882,0.005172,1,0.009861,0.005174,1,0.009858,0.005182,1,0.009852,0.005185,1,0.00985$, $0.005189,1,0.009843,0.005194,1,0.009838,0.005197,1,0.009836,0.005201,1,0.009803,0.00$ $5205,1,0.009794,0.005221,1,0.009776,0.005229,1,0.009758,0.005244,1,0.009749,0.005259$ $, 1,0.009735,0.00526,1,0.009733,0.005278,1,0.009705,0.00528,1,0.009686,0.005289,1,0.00$ 
$9675,0.005291,1,0.009668,0.005292,1,0.009663,0.005293,1,0.009656,0.005301,1,0.009637$ $, 0.005303,1,0.009632,0.005318,1,0.009615), . \operatorname{Dim}=c(39,3)), N=39)$

- Results:

\begin{tabular}{|l|l|l|l|l|l|l|l|}
\hline node & mean & sd & $2.50 \%$ & median & $97.50 \%$ & start & sample \\
\hline kapa & 113400 & 282900 & 44.89 & 7326 & 819600 & 1001 & 200000 \\
\hline kapa1 & 8.772 & 2.949 & 3.804 & 8.899 & 13.62 & 1001 & 200000 \\
\hline neta & 1.064 & 0.5651 & 0.1124 & 1.088 & 1.992 & 1001 & 200000 \\
\hline sigma & 0.01989 & 0.02722 & 0.01012 & 0.01408 & 0.06254 & 1001 & 200000 \\
\hline
\end{tabular}




\section{References}

[1] Suresh S., Fatigue of materials. Cambridge University Press. Cambridge. 1998.

[2] Fuchs H. O., Stephens R. I. , Metal Fatigue in Engineering, John Wiley \& Sons, New York, 1980.

[3] Boller C., "Ways and Options for Aircraft Structural Health Management", Journal of Smart Material and Structure, 10, 2001, pp. 432-440.

[4] Irving P. E., "Fatigue-Based Assessments in Aircraft Structures - Designing and Retaining Aircraft Structural Integrity", Ed. David Lidbury and Peter Hirsch, Methods for the Assessment of Structural Integrity of Components and Structures, by The Institute of Materials, Minerals and Mining, Maney Publishing, London, 2003, pp. 69-102.

[5] "World Aircraft Accident Survey", Flight International, January 2000.

[6] Wöhler A., "Wöhler's Experiments on the Strength of Metals," Engineering, August 23, 1867, pp. 160.

[7] Griffith A. A., "The Phenomena of Rupture and Flow in Solids," Trans. $R$. Soc. (Lond.), Vol. A221, 1920, pp. 163.

[8] Haigh B. P., "The Relative Safety of Mild and High-Tensile Alloy Steels under Alternating and Pulsating Stresses," Proc. Inst. Automob. Eng., Vol. 24, 1929/1930, pp. 320.

[9] Miner M.A., "Cumulative Damage in Fatigue," Trans. ASME, J. Appl. Mech., Vol. 67, Sept. 1945, pp. A159.

[10] Palmgren A., "Die Lebensdauer von Kugellagern," ZDVDI, Vol. 68, No 14, 1924, pp. 339.

[11] Irwin G.R., "Analysis of Stresses and Strains Near the End of a Crack Traversing a Plate," Trans. ASME, J. Appl. Mech. Vol. 24, 1957, pp. 361.

[12] Manson S.S., "Discussion of Ref. 14", Trans. ASME, J. Basic Eng., Vol. 84, No 4, Dec. 1962, pp. 537.

[13] Tavernelli J.F., Coffin L.F., Jr., "Experimental Support for Generalized Equation Predicting Low Cycle Fatigue," Trans. ASME, J. Basic Eng., Vol. 84, No 4, Dec. 1962, pp. 533. 
[14] Paris P.C., Erdogan F., "A Critical Analysis of Crack Propagation Laws," Trans. ASME, Vol. D85, 1963, pp. 528-534.

[15] Szolwinski M.P., Farris T.N., "Linking Riveting Process Parameters to the Fatigue Performance of Riveted Aircraft Structures", Journal of Aircraft, Vol. $37, \mathrm{~N}^{\mathrm{o}}$ 1, January-February 2000.

[16] Graziano W.D., Fitch G.E., Jr., "Initiation and Growth of Fatigue Cracks in and residual Strength of the F-100 Wing," Damage Tolerance in Aircraft Structures, ASTM STP 486, American Society for Testing and Materials, 1971, pp. 144-163.

[17] Kaplan M.P., Wolff, T.A., "Life Extension and Damage Tolerance of Aircraft", February 10, 2005, <http://www.aviationtoday.com/reports.htm>.

[18] Stone M., "Fatigue and Fail-Safe Design of a New Jet Transport Airplane", Fatigue Design Procedures, Proceedings of the $4^{\text {th }}$ Symposium of the International Committee on Aeronautical Fatigue, E. Gassner and W. Schutz, Ed., Pergamon Press, London, 1969, pp. 1-65.

[19] U.S. Department of Transportation, Damage Tolerance Assessment Handbook, Report N DOT-VNTSC-FAA-93.13.I, 1993, pp. 1-11.

[20] Federal Aviation Administration, 25.572 Subpart C, Part 25-Airworthiness Standards: Transport Category Aircraft, Federal Aviation Regulations, Washington, D.C.

[21] Federal Aviation Administration, Advisory Circular 25.571-1A, Damage Tolerance and Fatigue Evaluation of Structures, Washington, D.C.

[22] Lincoln J.W., Risk Assessment of an Aging Military Aircraft", Journal of aircraft, Vol. 22, $\mathrm{N}^{\mathrm{o}}$ 8, August 1985.

[23] Berens A.P., Burns J.C. and Rudd J. L., "Risk Analysis for Aging Aircraft Fleets," Structural Integrity of Aging Airplanes, S.N. Alturri et al., Ed., Berlin, 1991, pp. 37-51.

[24] Civil Aviation Authority, "CAP 681-Global Fatal Accident Review 19801996”, 1998, < http://www.caa.co.uk/docs/33/CAP681.PDF>.

[25] McCrone Atlas of Microscopic Particles, "Aluminum 7075", 2005, $<$ http://www.mccroneatlas.com/viewer/?PARTICLE_ID=1096>.

[26] "Nasgro 4.0 Manual", September 2002. NASA Johnson Space Center and Southwest Research Institute ${ }^{\mathrm{TM}}$ 
[27] Newman J.C. Jr., FASTRAN-II - A Fatigue Crack Growth Structural Analysis Program, NASA Technical Memorandum 104159, Langley Research Center, Hampton, VA 23665, USA, 1992.

[28] Schijve J., "Four Lectures on Fatigue Crack Growth", Engineering Fracture Mechanics, vol 11, 1979, pp.167-221

[29] Schijve J., "Observation on the Prediction of Fatigue Crack Growth Propagation Under Variable-Amplitude Loading." Fatigue Crack Growth Under Spectrum Loads, ASTM STP 595, American Society for Testing and Materials, 1976, pp. 3-23.

[30] eFunda, "Linear Elastic Fracture Mechanics", 2006, $<$ http://www.efunda.com/formulae/solid_mechanics/fracture_mechanics/fm_1 efm.cfm>

[31] Technical Data Analysis, Inc. (TDA) <http://www.tda-i.com/index.html $>$

[32] Collins J.A., Mechanical Design of Machine Elements and Machines, John Wiley \& Sons, Inc., New York, 2003.

[33] Schijve J., "Fatigue of Structures and Materials in the $20^{\text {th }}$ century and the State of the Art", International Journal of Fatigue, vol 25, 2003, pp. 679-702.

[34] Callister W.D. Jr., Materials Science and Engineering an Introduction, John Wiley \& Son, Inc., New York, 2000.

[35] Jacobs J.A., Kilduff, T.F., Engineering Materials Technology: Structures, Processing, Properties, and Selection, Prentice Hall, New Jersey, 2005.

[36] Bannantine J.A., Comer J.J., and Handrock J.L., Fundamentals of Metal Fatigue Analysis, Prentice Hall, New Jersey, 1990.

[37] Hardrath, H. F., et al., "Recent Developments in Analysis of Crack Propagation and Fracture of Practical Materials" In: Fracture Mechanics, by Perrone, N., Liebowitz, H., Mulville, D. and Pilkey, W., University Press of Virginia, Charlottesville, 1978, pp. 347-364.

[38] Dowling N.E. Mechanical Behavior of Materials, New Jersey. Prentice Hall, 1998.

[39] Broek D., Elementary Engineering Fracture Mechanics, Martinus Nijhoff Publisher, Dordrecht, 1987.

[40] Shaffer J.P. et al., The Science and Design of Engineering Materials, McGraw-Hill, Boston, 1999. 
[41] Allen F.C., "Effect of Thickness on the Fracture Toughness of 7075 Aluminum in the T6 and T73 Conditions" In: Damage Tolerance in Aircraft Structures, STP 486, by American Society for Testing and Materials, Philadelphia, 1970, pp.16-38.

[42] Tong Y.C., Literature Review on Aircraft Structural Risk and Reliability Analysis., DSTO-TR-1110, DSTO Aeronautical and Maritime Research Laboratory, Melbourne, Australia, February 2001.

[43] Hovey P.W., Berens A.P. and Skinn, D.A., Risk Analysis for Aging Aircraft Volume 1 - Analysis, Flight Dynamics Directorate, Wright Laboratory, Wright-Patterson AFB, OH 45433-6553, October 1991.

[44] Melchers R.E., Structural Reliability Analysis and Prediction, John Wiley \& Sons Ltd, Chichester, 1999.

[45] Farahmand B., Fatigue and Fracture Mechanics of High Risk Parts: Application of LEFM \& FMDM, International Thomson Publishing, New York, 1997.

[46] Gassner E., "Strength Experiments Under Cyclic Loading in Aircraft Structures", Luftwissen, 1939, 6, pp. 61-64.

[47] Teichmann A., "Basic Consideration on Fatigue Durability in Service", Jahrbuch der Deutschen Luftfahrtforschung, 1941, 1, pp 467-471.

[48] Seewald F., "Measurements with a Glass Scratching Recorder of the German Institute for Aeronautics", Maschinenbau, 1931, 10, pp. 725-727. (in german)

[49] Taylor J., "Measurements of Gust Loads in Aircraft", J Roy Aero Soc, 1953, 57 , pp. $78-88$.

[50] Omega.com, "The Strain Gage", 2006, < http://www.omega.com/literature/transactions/volume3/strain.html >.

[51] ASME E-1049, Standard Practices for Cycle Counting in Fatigue Analysis, American Society Of Mechanical Engineers, 1997.

[52] Molent L., "A Review Of A Strain And Flight Parameter Data Based Aircraft Fatigue Usage Monitoring System" USAF ASIP Conference San Antonio, TX, 3 -5 Dec 1996. www.dsto.defence.gov.au/publications/2799/DSTO-162.pdf.

[53] Hurtado J.L., Hoffman P., "Airframe Integrity Based on Bayesian Approach", The International Symposium on Product Quality \& Integrity, San Diego, USA, January 2006.

[54] Artley M.E., Probabilistic Damage Tolerance Method for Metallic Aerospace Structure, Wright-Patterson AFB OH 45433-6553, September 1989. 
[55] Rudd J.L., Gray, T.D., "Quantification of Fastener-Hole Quality”, Journal of Aircraft, 15, 3, 1978, pp.143-147.

[56] Yang J.N., Manning, S.D., "Statistical Distribution of Equivalent Initial Flaw Size", Proc. 1980 Annual Reliability and Maintainability Symposium, 1980, pp.112-120.

[57] Funatogawa H., Ohnabe, H., and Yang, J.N., "Monte Carlo Simulation for Probabilistic fatigue analysis of Jet Engine Disks", $4^{\text {th }}$ International Conference on Structural Safety and Reliability, Doc. N ${ }^{\circ}$ 1518, 1985.

[58] LaRiviere S.G., Thompson J., "Development of Reliable NDI Procedures for Airframe Inspection". NATO RTO Meeting Proc. 10: Airframe Inspection Reliability under Field/Depot Conditions. Paper 7.

[59] Sander M., "Comparison of Fatigue Crack Growth Concepts with respect to Interaction Effects", ECF15 Proceedings, Stockholm, Sweden, August 1113, 2004.

[60] Walker K., "The Effect of stress Ratio During Crack Propagation and Fatigue for 2024-T3 and 7075-T6 Aluminum", Effects of Environment and Complex Load History on Fatigue Life, ASTM STP 462, American Society for testing and Materials, 1970, pp. 1-14.

[61] Brock D., Schijve, J., "The Influence of the Mean Stress on the Propagation of Fatigue Cracks in Aluminum Alloy Sheet", NRL-TR M.2111, National Luchten Ruimtevaart/Aboratorium, January, 1963.

[62] McMillan J.C., Pelloux, R.M., "Fatigue Crack Propagation under Program and Random Loads, Fatigue Crack propagation", ASTM STP 415, American Society for testing and Materials,1967, pp 505.

[63] Erdogan F., Crack propagation Theories, NASA CR901, National Aeronautics and Space Administration, October, 1967.

[64] Hartman A., et al., "Some Tests on the Effect of the Environment on the Propagation of Fatigue Cracks in Aluminum Alloys", NRL-TN M.2182, National Lucht-en Ruimtevaart/Aboratorium, January, 1967.

[65] Forman R.G., Kearney V.E., and Engle R.M., "Numerical Analysis of Crack Propagation in a Cyclic-Loaded Structure," Trans. ASME, J. Basic Eng., Vol. D89, No. 3, 1967, pp. 459-464.

[66] Fatigue Crack Growth - An Engineers Toolbox calculation module. www.engineerstoolbox.com/

[67] Newman J.C., Jr., "A Crack-Closure Model for Predicting Fatigue Crack Growth under Aircraft Spectrum Loading," Methods and Models for 
predicting fatigue Crack Growth under Randon Loading, ASTM STP 748, J. B. Chang and C. M. Hudson, Eds., American Society for Testing and Materials, 1981, pp. 53-84.

[68] Elber W., "Fatigue Crack Growth under Cyclic Tension", Engineering Fracture Mechanics, vol. 2, No 1, July 1970, pp. 37-45.

[69] Elber W., "The significance of Fatigue Crack Closure," Damage Tolerance in Aircraft Structures, ASTM STP 486, American Society for Testing and Materials, Philadelphia, 1971, pp. 230-242.

[70] Schijve J., "Fatigue Crack Closure: Observation and Technical Significance", Mechanics of Fatigue Crack Closure. ASTM STP 982, J. C. Newman, Jr. and W. Elber, Eds., American Society for Testing and Materials, Philadelphia, 1988, pp. 5-34.

[71] Clerivet A., Bathias C., "Study of Crack Tip Opening under Cycling Loading Taking into Account the Environment and R Ratio" - Eng. Fract. Mech., 1979, vol. 12, n 4, pp. 599-611.

[72] Zhang S., et al., "Crack propagation studies on Al-7475 on the basis of constant amplitude and selective variable amplitude loading histories",Fatigue \& Fracture of Engineering Materials \& Structures, vol.10, no.4, 1987. p. 315332.

[73] Kurihara M., Kato A. and Kawahara, M., "Current Research of Fatigue Cracks", The Japanese Society of Materials Science, MRS, Vol. 1, 1985, pp. 217-233.

[74] Ray A., Patankar R., "Fatigue Crack Growth under Variable-Amplitude Loading: Part I - Model formulation in state-space setting", Applied mathematical modeling, 25, 2001. 979-994.

[75] Ray A., Patankar R., "Fatigue Crack Growth under Variable-Amplitude Loading: Part II - Code development and Model Validation", Applied mathematical modeling, 25, 2001, pp 995-1013.

[76] Newman J.C., Jr., "A Crack Opening Stress Equation for Fatigue Crack Growth", International Journal of Fracture, 24, 1984, R131-R135.

[77] Harter J.A. AFGROW users Guide and Technical Manual, Report No AFRLVA-WP-TR-2004-XXXX, Air Force Research Laboratory, WPAFB OH, 45433-7542, 2004.

[78] Kujawski D., "Utilization of Partial Crack Closure for fatigue Crack Growth Modeling", Engineering Fracture Mechanics, 69, 2002, pp. 1315-1324.

[79] Caiani C. Accelerated Tests. Reliasoft, Brasil, 2002. 
[80] Reliasoft Corporation, "Life Distribution and Life-Stress Models", 2006, $<$ http://www.weibull.com/AccelTestWeb/life_distribution_and_stress_life_m odels.htm $>$.

[81] Keyser C.A., Materials science in Engineering, Columbus, Charles E. Merrill Publishing Co., 1986.

[82] Relex Software Corporation, "Failure and Repair Distributions, Reliability Articles", 2006, <http://www.relex.com/resources/art/art_distrib.asp >.

[83] Weber H., "De pulsa resorptione auditu et tactu", Annotationes anatomicaeet physiologicae, Leipzig (Germany): Koehler, 1834.

[84] Fechner G.T., "Elemente der Psychophysik”, Leipzig (Germany): Breitkopf und Härtel, 1860.

[85] Galton F., "The geometric mean, in vital and social statistics", Proceedings of the Royal Society, 29:1897, pp. 365-367.

[86] McAlister D., "The law of the geometric mean", Proceedings of the Royal Society, 29, 1897, pp. 367-376.

[87] Limpert E., Stahel W.A, and Abbt, M., "Log-normal Distributions across the Sciences: Keys and Clues”, BioScience, 51, (5), 2001, pp. 341-352.

[88] Palisade Corporation, Guide to Using@,RISK, Risk Analysis and Simulation Add-In for Microsoft ${ }^{\circledR}$ Excel, Version 4.5, New York, 2002.

[89] Annis Ch., "Goodness-of-Fit test for Statistical Distributions, Statistical engineering”, 2006, < http://www.statisticalengineering.com/goodness.htm >.

[90] Owen W. J., Padgett, W.J., “A Birnbaum-Saunders accelerated life model”, IEEE Transactions on Reliability, vol. 49, 2000, pp. 224.229.

[91] ReliaSoft Corporation, "Inverse Power Law Relationship", 2006, $<$ http://www.weibull.com/AccelTestWeb/inverse_power_law_relationship_int roduction.htm $>$.

[92] Meagher P., "Implement Bayesian inference using PHP: Part 2 -Solving parameter estimation problems", 2004, <http://www128.ibm.com/developerworks/web/library/wa-bayes2/>.

[93] ReliaSoft Corporation, "Inverse Power Law-Lognormal”, 2006, $<$ http://www.weibull.com/AccelTestWeb/ipl_lognormal.htm $>$.

[94] ReliaSoft Corporation, "MLE (Maximum Likelihood) Parameter Estimation for Complete Data", 2006, < http://www.weibull.com/LifeData Web/mle_for_complete_data.htm $>$. 
[95] Metropolis N., et al., "Equation of State Calculations by Fast Computing Machines", J. Chem. Phys., 21, 1953, pp. 1087-1092.

[96] Berg B.A., "Introduction to Markov Chain Monte Carlo Simulations and their Statistical Analysis", < http://www.worldscibooks.com/physics/5602.html>.

[97] Walsh B., Markov Chain Monte Carlo and Gibbs Sampling, Lecture Notes for EEB 596z, 2002.

[98] "Markov Chain Monte Carlo", $<$ http://www.ecpag.org/presentations/1999/presentations2/tsld009.htm >.

[99] Metropolis N., Ulam S., "The Monte Carlo method", J. Amer. Statist. Assoc,44, 1949, pp. 335-341.

[100] Hastings, W.K., "Monte Carlo sampling methods using Markov Chains and their applications", Biometrika, 57, 1970, pp. 97-109.

[101] Chib S., Greenberg E., "Understanding the Metropolis-Hastings algorithm", American Statistician, 49, 1995, pp 327-335.

[102] Geman S., Geman, .D., "Stochastic relaxation, Gibbs distribution and Bayesian restoration of images", IEE Transactions on Pattern Analysis and Machine Intelligence, 6: 1984, pp. 721-741.

[103] Tierney L., "Markov chains for exploring posterior distributions (with discussion)", Ann. Statist., 22, 1994, pp 1701-1762.

[104] BUGS, "Bayesian inference using Gibbs Sampling", <http://www.mrcbsu.cam.ac.uk/bugs/Welcome.html>.

[105] Fogel D., Evolutionary Computation, New York. IEEE Press. 1995.

[106] "Neural and evolutionary techniques for natural language processing", $<$ http://cnts.uia.ac.be/ kool/nnga.html $>$.

[107] Holland J.H., Adaptation in natural and artificial systems, Ann Arbor: The University of Michigan Press, 1975.

[108] "Genetic Algorithm", <http://www.bridgeport.edu/sed/projects/449/ Fall_2000/fangmin/chapter4.htm>.

[109] Blickle T., "Tournament selection", <http://www.iop.org/Books/CIL/HEC/ pdf/ECC2_3.PDF>.

[110] Baker J.E., "Reducing bias and inefficiency in the selection algorithm", Proc. Of the second International Conference on Genetic Algorithms, Ed. J.J. 
Grefenstette, Massachusetts Institute of Technology, Cambridge, MA. pp.1418. 1987.

[111] Mühlenbein H., Schlierkamp-Voosen D., "Predictive Models for the Breeder Genetic Algorithm: I. Continuous Parameter Optimization", Evolutionary Computation, 1 (1), pp. 25-49, 1993.

[112] De Jong K.A., An analysis of the behavior of a class of genetic adaptive systems, Ph.D thesis, University of Michigan. Diss. Abstr. Int. 36(10), 5140B, University Microfilms No. 76-9381, 1975.

[113] Shaffer J.D., et al., "A study of control parameters affecting online performance of genetic algorithms for function optimization", In Shaffer, pp. 51-60, 1989.

[114] Grefenstette J.J., "Optimization of control parameters for genetic algorithms", IEEE transactions of Systems, Man and Cybernetics SMC -16 (1), 122-128. 1986.

[115] Mühlenbein H., "The Breeder Genetic Algorithm - a provable optimal search algorithm and its application", Colloquium on Applications of Genetic Algorithms, IEE 94/067, London, 1994.

[116] Pohlheim H., Genetic and Evolutionary Algorithm Toolbox for use with Matlab (GEATbx), Copyright (C) 1996. Germany.

[117] Michalewicz Z., Genetic Algorithms + Data Structures = Evolution Programs, New York. Springer-Verlag. 1992.

[118] Marczyk A., "Genetic Algorithms and Evolutionary Computation", April 2004, <http://www.talkorigins.org/faqs/genalg/genalg.html\#limitations>.

[119] Niedermayer D., "An Introduction to Bayesian Networks and their Contemporary Applications", 1988. <http://www.niedermayer.ca/papers/ bayesian/>.

[120] Apostolakis G., Bayesian Methods in Risk Assessment, Advances in Nuclear Science and Technology, J. Lewins and M. Becker, ed., Plenum Press, 1981.

[121] "Bayesian inference", 2006, <http://en.wikipedia.org/wiki/Bayesian _statistics>,

[122] Mosleh A., ENRE 665 Course Handouts. University of Maryland, College Park, MD, 2000.

[123] Groen F., "R-DAT Plus-Bayesian data analysis package for risk analysts", $<$ http://www.prediction-technologies.com $>$. 
[124] Ayyub B., ENCE 615 Course Handouts. University of Maryland, College Park, MD, 2003.

[125] Nguyen O., et al, "A cohesive model of fatigue crack growth", International Journal of Fracture, 110: 351-369, 2001.

[126] Modarres M., Risk Analysis in Engineering: Techniques, Tools, and Trends, CRC, 2006.

[127] Isukapalli S., Uncertainty Analysis of Transport-Transformation Models, $\mathrm{PhD}$ Dissertation, Rutgers, The State University of New Jersey, 1999. 


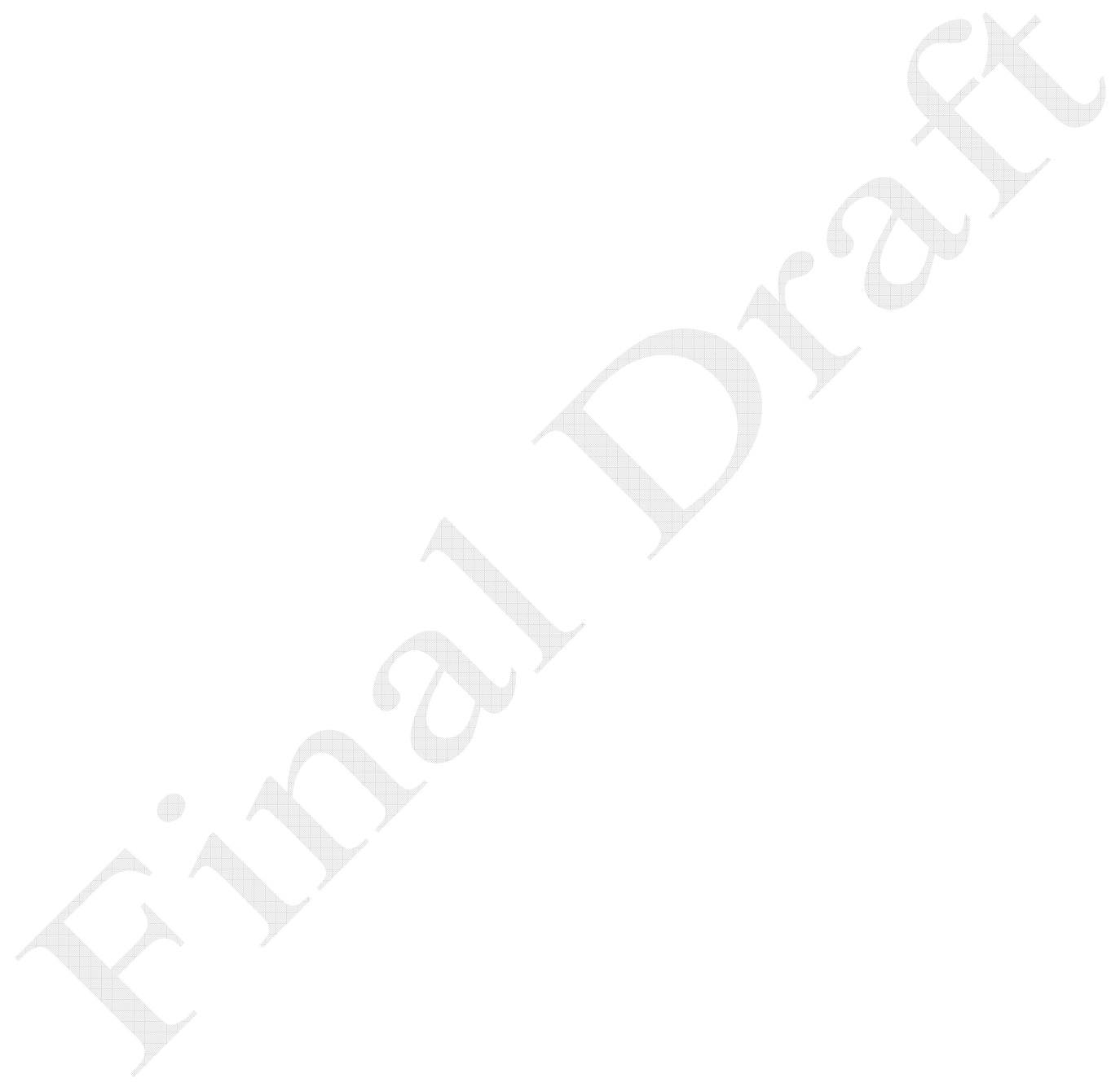

